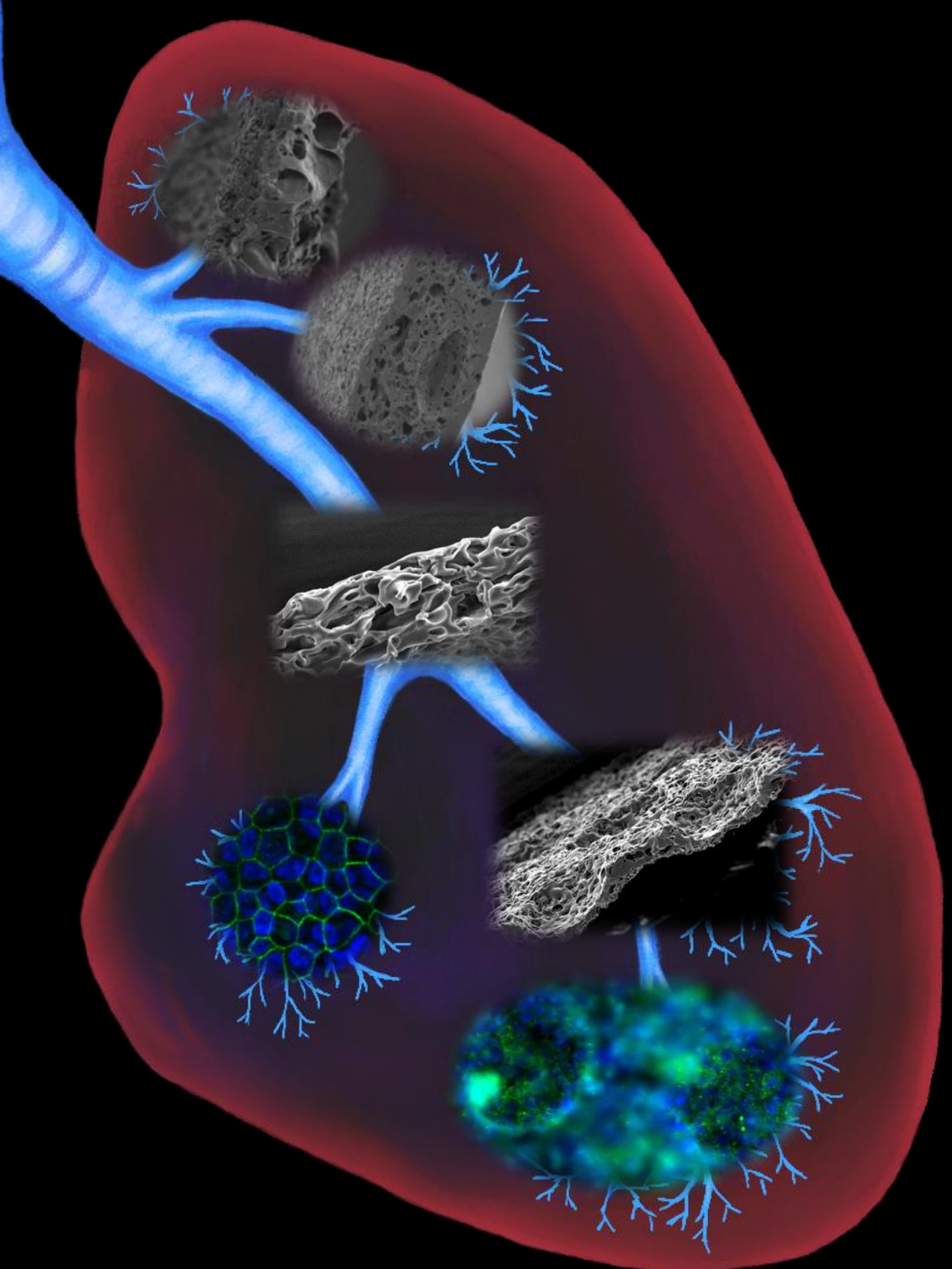

\title{
Poly(trimethylene carbonate)-based membranes for biomimetic lung epithelial-endothelial models
}

Thijs Pasman 



\section{POLY(TRIMETHYLENE CARBONATE)-BASED MEMBRANES FOR BIOMIMETIC LUNG EPITHELIAL-ENDOTHELIAL MODELS}

Thijs Pasman 



\title{
POLY(TRIMETHYLENE CARBONATE)-BASED \\ MEMBRANES FOR BIOMIMETIC LUNG EPITHELIAL-ENDOTHELIAL MODELS
}

\author{
DISSERTATION
}

\author{
to obtain \\ the degree of doctor at the University of Twente, \\ on the authority of the rector magnificus, \\ prof. dr. ir. A. Veldkamp, \\ on account of the decision of the Doctorate Board \\ to be publicly defended \\ on Wednesday the $8^{\text {th }}$ of December 2021 at 16.45 hours
}

by

Thijs Pasman

born on the $5^{\text {th }}$ of April, 1990

in Winterswijk, The Netherlands 
This dissertation has been approved by:

Supervisors

Prof. Dr. D.W. Grijpma

Prof. Dr. D. Stamatialis

Co-supervisor

Dr. A.A. Poot

The research was performed in the research group of Biomaterials Science and Technology at the University of Twente, The Netherlands, and was financially supported by The Lung Foundation Netherlands (Project: Microengineered 3D analogues of alveolar tissue for lung regeneration, number 6.1.14.010).

The printing of this thesis was sponsored by:

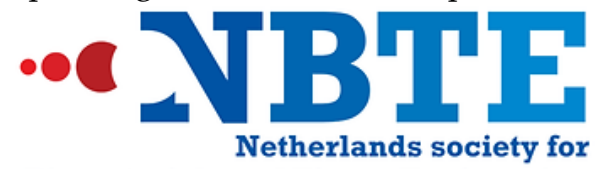

Biomaterials and Tissue Engineering

Nederlandse vereniging voor Biomaterialen en Tissue Engineering

Netherlands society for Biomaterials and Tissue Engineering

Cover design: $\quad$ Lisa Rosendaal and Thijs Pasman

Printed by: Ipskamp Printing

Lay-out: $\quad$ Thijs Pasman

ISBN: $\quad$ 978-90-365-5228-8

DOI: $\quad 10.3990 / 1.9789036552288$

Cover: SEM images of PTMC membranes and fluorescent microscopy images of Calu-3 cells and LMVECs on PTMC membranes symbolising the project, from TIPS and LIPS to EIPS, micromoulding and cell culturing.

(C) 2021 Thijs Pasman, The Netherlands. All rights reserved. No parts of this thesis may be reproduced, stored in a retrieval system or transmitted in any form or by any means without permission of the author. Alle rechten voorbehouden. Niets uit deze uitgave mag worden vermenigvuldigd, in enige vorm of op enige wijze, zonder voorafgaande schriftelijke toestemming van de auteur. 


\section{Graduation Committee:}

Chair / secretary:

Supervisors:

Co-supervisor:

Committee Members:
Prof. Dr. J.L. Herek

University of Twente, The Netherlands

Prof. Dr. D.W. Grijpma

University of Twente, The Netherlands

Prof. Dr. D. Stamatialis

University of Twente, The Netherlands

Dr. A.A. Poot

University of Twente, The Netherlands

Prof. Dr. R.K. Truckenmüller

Maastricht University, The Netherlands

Prof. Dr. P.S. Hiemstra

Leiden University, The Netherlands

Prof. Dr. P.C.J.J. Passier

University of Twente, The Netherlands

Prof. Dr. Ir. L.I. Segerink

University of Twente, The Netherlands

Dr. L. De Bartolo

Institute on Membrane Technology of the National Research Council of Italy, Italy 


\section{- Table of content -}

Chapter 1: General introduction

Chapter 2: Flat and microstructured polymeric membranes in 16 organs-on-chips

Chapter 3: Fabricating porous, photo-crosslinked 60 poly(trimethylene carbonate) membranes using temperature-induced phase separation

Chapter 4: Porous and form-stable poly(trimethylene carbonate) 78 membranes prepared by liquid-induced phase separation and photo-crosslinking

Chapter 5: Development of porous and flexible PTMC membranes 100 for in vitro organ models fabricated by evaporationinduced phase separation

Chapter 6: Development of an in vitro airway epithelial-endothelial 148 cell culture model on a flexible porous poly(trimethylene carbonate) membrane based on Calu-3 airway epithelial cells and lung microvascular endothelial cells

Chapter 7: $\quad$ Development of flexible porous PTMC membranes with 186 microwells for an in vitro airway epithelial-endothelial cell culture model

Chapter 8: Conclusions and future outlook 
List of abbreviations

List of publications

About the author

Acknowledgements and final words

250 



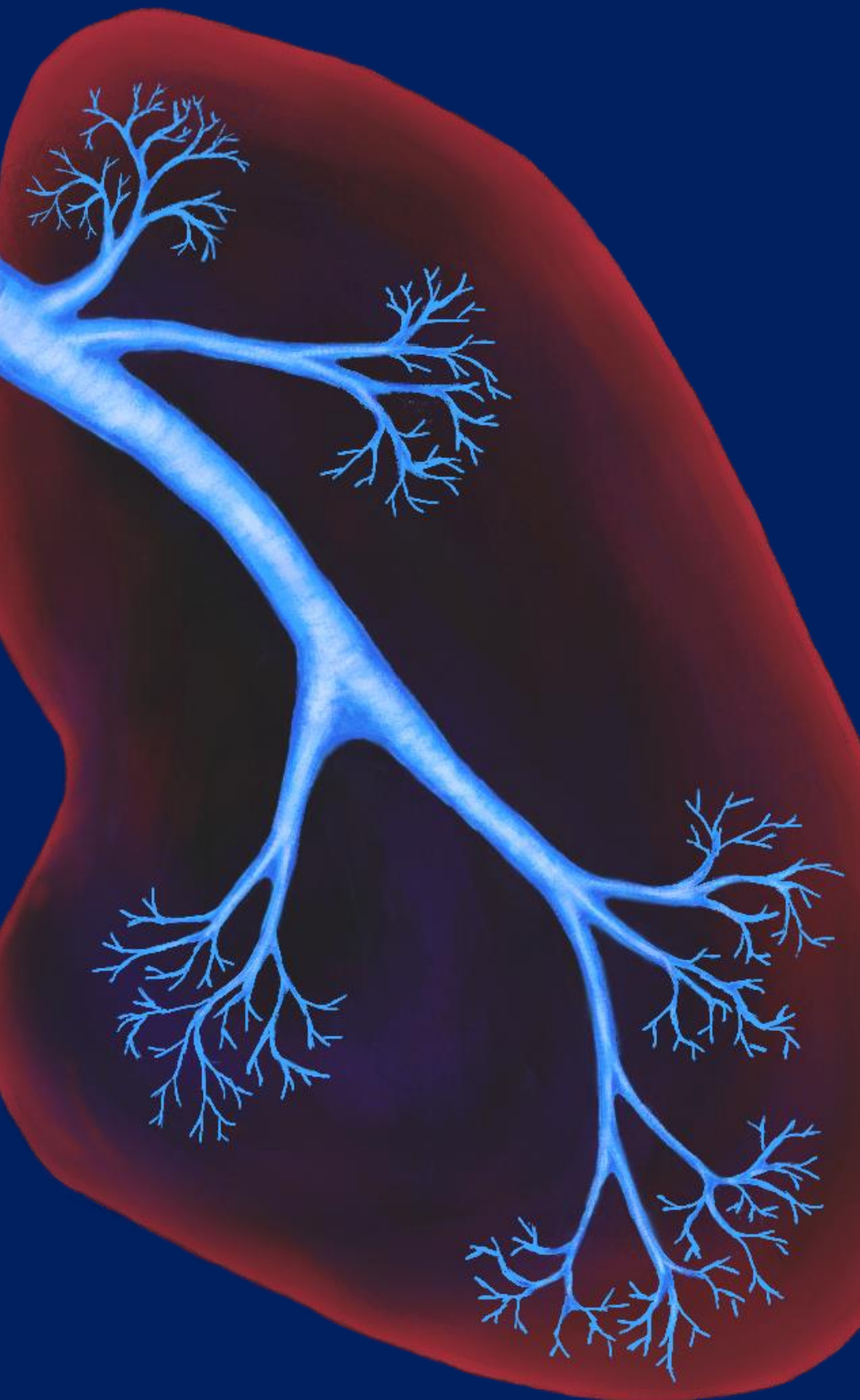




\section{- Chapter 1 -}

\section{General introduction}

Thijs Pasman, Dimitrios Stamatialis and André A. Poot

Technical Medical (TechMed) Centre, Department of Biomaterials Science and Technology, Faculty of Science and Technology, University of Twente, 7522 NB Enschede, The Netherlands 
Lungs are vital organs, solely responsible in many animals, including humans, for enabling the uptake of oxygen by the erythrocytes in the blood while also allowing carbon dioxide, a cytotoxic by-product of cell metabolism, to leave the body. Inhaled air flows from the trachea in increasingly smaller ducts, i.e. several sets of bronchi and bronchioles, which branch out from the trachea (Figure 1). Eventually, air reaches the alveoli, small spherical structures with a thin cell lining of lung epithelial cells surrounded by a basement membrane, i.e. a thin extracellular matrix (ECM), and capillaries. This alveolar-capillary interface is where the vital exchange of oxygen and carbon dioxide takes place, which is necessary for the functioning of our cells. Internal factors such as genetic deficiencies can lead to serious diseases, for example, cystic fibrosis. Additionally, external factors such as antigens, pathogens and pollutants can also result in disorders since the lungs are in direct contact with the air. Some lung diseases can be triggered by both internal and external factors, such as lung cancer and chronic obstructive pulmonary disease (COPD). Thus, many factors can compromise the functioning of the lungs, and by extension, the alveoli.

Unsurprisingly, lung diseases are among the major causes of death [2]. Moreover, the recent rise of new respiratory viruses such as SARS-CoV2 , i.e. the coronavirus that causes Covid-19, and the current pandemic because of the latter present an unparalleled impact on society. Consequently, there is an unprecedented focus on lung research and need for proper models to perform that research. However, traditional in vitro and in vivo models, i.e. static cell cultures in e.g. Petri dishes, well plates and Transwell ${ }^{\circledR}$ cell culture inserts, and animal models, respectively, have significant disadvantages. There is an increasing resistance towards the use of animal models due to ethical reasons and because they often do not provide good predictive data on how new medicines and treatments will function in humans. Many in vitro models do not resemble the native tissue since they cannot provide the cells with relevant physiological stimuli such as airflow, fluid flow or mechanical stimulation. 


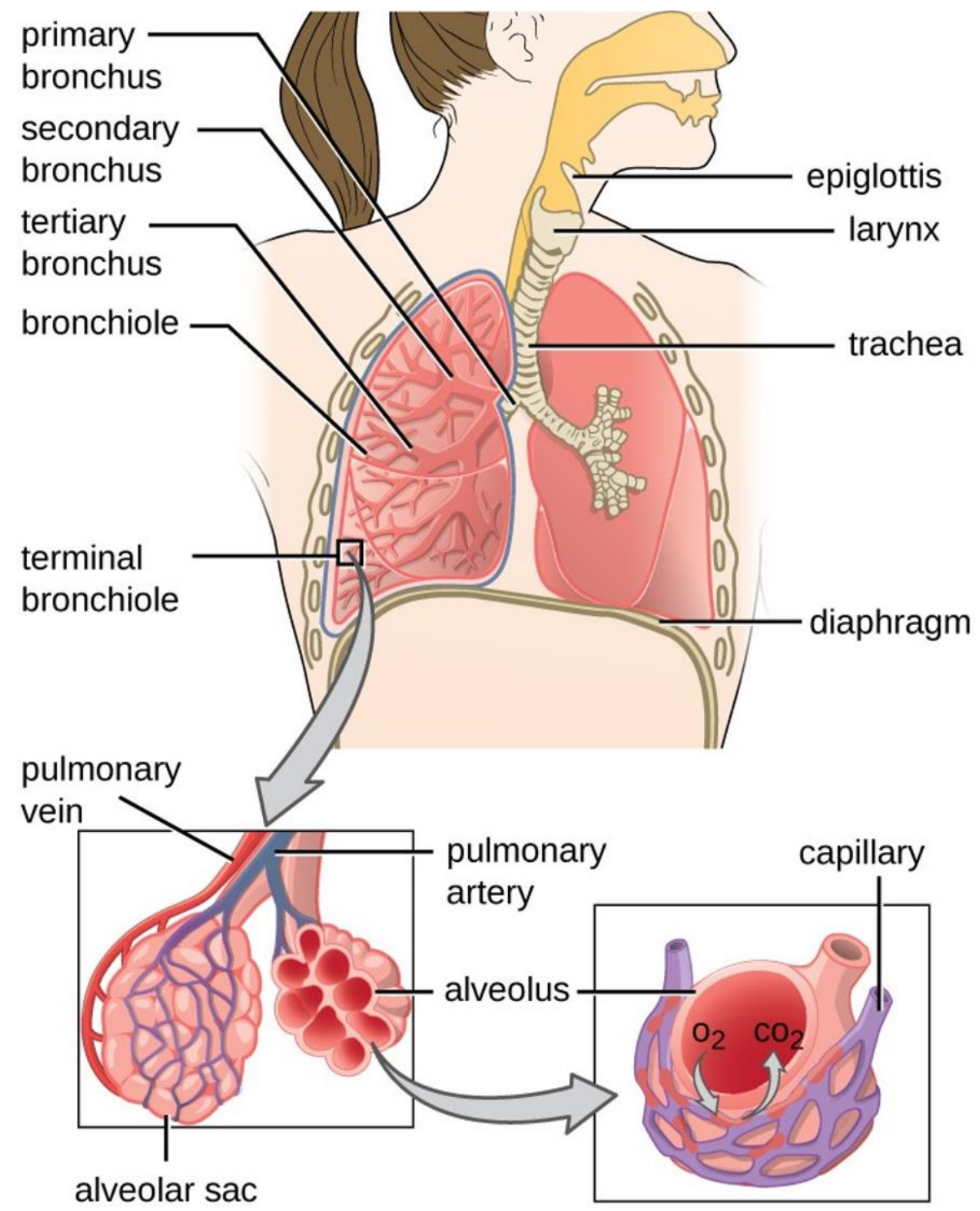

Figure 1. Anatomy of the lungs [1]. The trachea branches out into increasingly smaller ducts, i.e. bronchi, bronchioles and finally alveoli. In the latter, oxygen and carbon dioxide are exchanged between the alveoli and capillaries.

Lung epithelial cells are often cultured in Transwell ${ }^{\circ}$ inserts. These are small baskets in a well of a culture plate. Cells are cultured on a porous membrane on the bottom of the insert, which suspends the cells above the 
bottom of the well. This way, the lung cells can be exposed to air while receiving nutrients from the medium in the well via the porous membrane. The exposure to air is a great advantage of the inserts, but the model still lacks features such as airflow and mechanical stretch of the cells that mimics breathing. Moreover, the membranes on which the cells grow are usually made from poly(bisphenol-A-carbonate) (PC) or poly(ethylene terephthalate) (PET) [3], which have high elastic moduli (i.e. 2-2.4 GPa [4] and 2-3 GPa [5], respectively) compared to most natural tissues of which the moduli are in the range of a few $\mathrm{kPa}$ to MPa [6-9].

Thus, there is a need for the development of biomimetic lung epithelialendothelial models, such as the proposed alveolar-capillary model presented in Figure 2. In recent years, the development of organs-onchips (OOCs) paved the way to drastically alter traditional in vitro models. OOCs are small biomimetic in vitro models that introduce physiological stimuli, such as fluid flow and mechanical stress, to the cells [3]. Lungs-on-chips (LOCs) were the first OOCs to be developed [10]. Although there are currently multiple versions of LOCs, they generally resemble the Transwell@ insert models. Lung epithelial cells are cultured on a porous membrane in an air-filled compartment with a nutrient-rich fluid, usually cell culture medium, on the other side of the membrane in a separate compartment. A flow of air or medium can be created in the compartments, and some models also facilitate mechanical stretch of the membrane and cells, resembling the lung expansion during respiration. Although this is a great step towards biomimetic lung epithelialendothelial models, the properties of the membranes in these models are often overlooked. They often use stiff commercial PC or PET membranes or membranes made from poly(dimethyl siloxane) (PDMS) [3,11-16]. PDMS can be easily processed, and its E-modulus can have a wide range [17]. However, PDMS requires additional processing or coatings to enable cell growth. Moreover, PDMS does ad- and absorb small molecules, which makes (drug) studies complicated $[18,19]$. 
- Chapter 1 -

General introduction

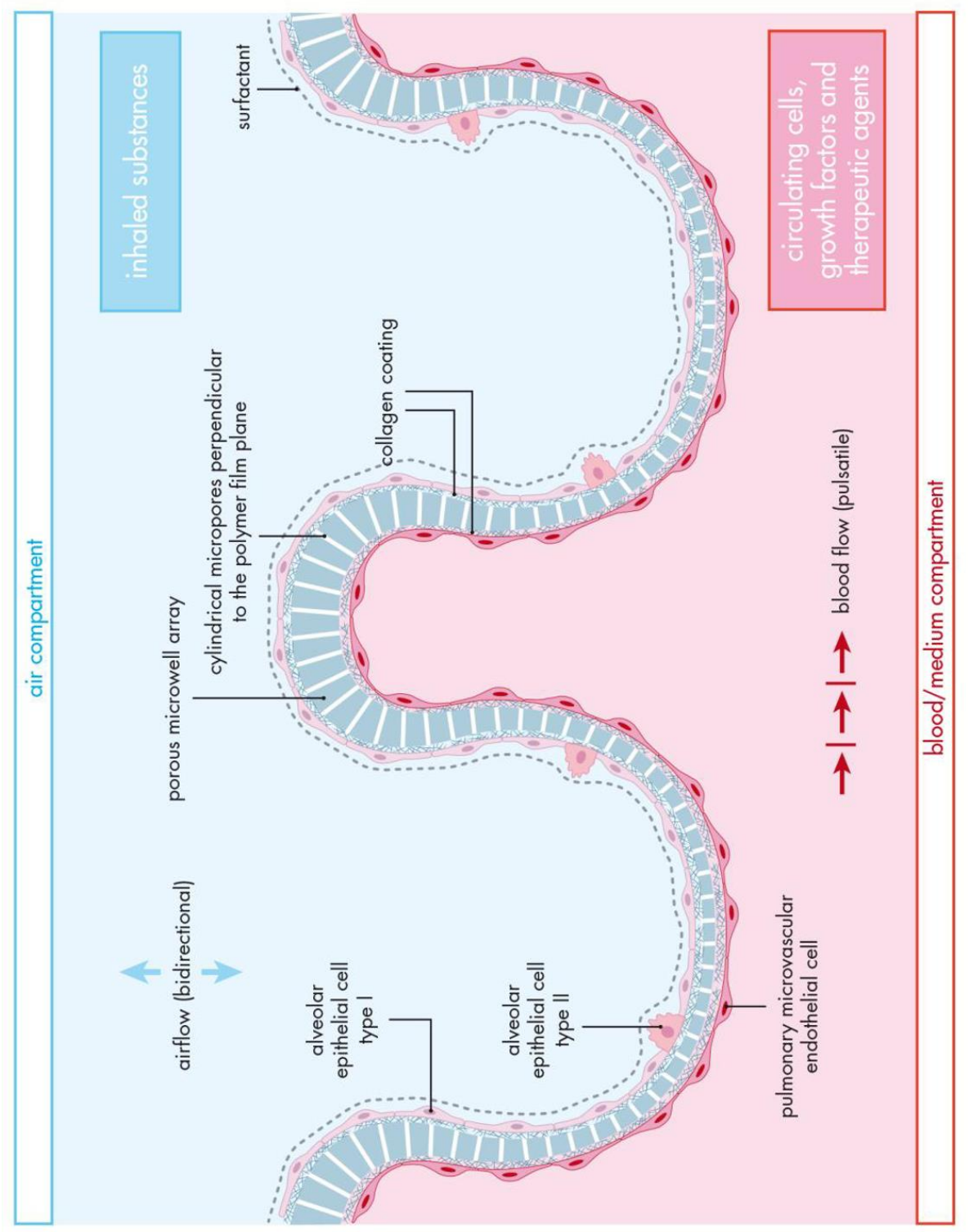


Figure 2. Schematic of a possible biomimetic alveolar-capillary interface. Primary alveolar epithelial cells and primary pulmonary microvascular endothelial cells are cultured on a porous membrane with microwells that resemble alveoli in both size and shape. The membrane is coated to facilitate the attachment and growth of the cells. In the compartment with the endothelial cells, a flow of a nutrientrich fluid such as cell culture medium is present. In contrast, in the compartment with the epithelial cells, i.e. the air compartment, air is flowed in and out of the microwells to mimic breathing. Different pathogens, such as pollutants, antigens and bacteria, can be added to the air- and/or fluid flow to investigate their role in alveolar pathologies. Conversely, substances and cells such as growth factors, therapeutic agents and immune cells can be added to examine their effects on maintenance and healing of the alveoli, and removal of pathogens.

Besides, most membranes in LOCs, as well as OOCs and in vitro models in general, are flat [3]. This is despite the evidence that large, threedimensional microstructures (up to several hundred micrometres in both height and width) do influence cell behaviour [3]. For example, attachment, proliferation and differentiation of cells are influenced by such microstructures, depending on the cell type and the shape and dimensions of the microstructures. Soscia et al. cultured salivary gland cell lines on PDMS films with microwells and reported e.g. better cell polarisation and signs of differentiation as the curvature of the microwells was increased [20]. Salivary glands contain many acini, small sac-like structures, resembling alveoli to some extent. As the curvature of the microwells increased, the microwells arguably became more acini-like. This probably triggered the cell response, which suggests that a more physiological shape of the membrane can lead to more physiological behaviour of cells. Similar effects could be expected from incorporating microwells in the membranes of lung models, such as suggested in Figure 2.

Thus, development of novel membranes for lung epithelial-endothelial models, which consider the above-mentioned influence of material and membrane properties on cell behaviour, is a relevant step towards making these models truly biomimetic. 


\section{Aim and outline of the thesis}

In this work, we describe the development of novel membranes for biomimetic lung epithelial-endothelial models. First, we establish the relevance of membrane properties in relation to cell behaviour. Then, we report on the development of membranes based on the polymer poly(trimethylene carbonate) (PTMC). This polymer has been used quite extensively in the past for the production of films, membranes and scaffolds for biomedical applications due to its advantageous properties for cell culturing, such as its transparency and good cytocompatibility [2127]. Moreover, it has good mechanical properties, such as a substantially lower E-modulus than PC and PET [25-31]. Besides these characteristics, the membranes should be porous and permeable to enable flow of nutrients through the membrane, as well as allow paracrine communication between cells on opposite sides of the membrane. However, the pore size should not allow cell migration. Furthermore, the membrane should also be mechanically stable to retain its porosity throughout the cell culturing. Thus, we present several studies in which we used different approaches to fabricate such membranes.

Chapter 2 reviews different aspects of porous polymeric membranes in OOCs to show the relevance and potential of membrane properties for organ models. First, it describes OOCs and LOCs compared to traditional Transwell ${ }^{\circledR}$ insert-based models. It then discusses different characteristics of materials such as hydrophilicity, porosity and surface topography, i.e. roughness and microstructures, which can influence cell behaviour. Cellular mechanisms involved in these cell responses are highlighted. Then, it gives an overview of the most commonly used polymers and techniques to fabricate porous membranes for OOCs, and presents advantages and disadvantages of these polymers and techniques as well as the membranes that are fabricated with them. Properties of the membranes, such as mechanical properties, are discussed in relation to the cells. Lastly, it focuses on techniques that involve micromoulding, which could result in more biomimetic membranes, i.e. by including 
microstructures that resemble a specific tissue, to improve in vitro models such as OOCs.

Chapters 3 - 5 describe the development and characterisation of PTMCbased membranes for use in in vitro lung models, such as LOCs. Different phase separation techniques are used to fabricate a variety of membranes with different properties such as pore size, porosity and water permeance.

Chapter 3 discusses the use of temperature-induced phase separation (TIPS) to fabricate porous PTMC-based membranes, which is inspired by previous work by Zant et al. [32]. The created membranes are subsequently treated with UV-light to crosslink them. The membranes are characterised, and the influence of cooling during crosslinking on these characteristics is investigated.

In Chapter 4, PTMC membranes are fabricated and tailored using liquidinduced phase separation (LIPS). This study builds upon earlier work from Papenburg et al. [23] and focuses on using poly(ethylene oxide) (PEO) and poly(vinyl pyrrolidone) (PVP) as pore formers in the membranes during LIPS. The effects of the molecular weight (MW) of $\mathrm{PEO}$ and the concentration of PEO and PVP on membrane properties are discussed. Furthermore, a novel approach is presented to enable photocrosslinking of these LIPS-based membranes.

Chapter 5 presents the development of PTMC membranes using evaporation-induced phase separation (EIPS), which has not been used before with PTMC. Effects of environmental conditions during membrane casting and EIPS (e.g. the temperature and humidity) and the composition of the polymer dope (e.g. the non-solvent type and amount, MW of PTMC, and inclusion of PEO) are discussed. Membranes are characterised and compared to commercial PET and PC membranes.

In Chapter 6, membranes developed in Chapter 5 are used for developing a novel in vitro airway epithelial-endothelial model with Calu-3 human lung epithelial cells and human lung microvascular endothelial cells (LMVECs). First, coating of the membrane is optimised. A co-culture of 
Calu-3 cells and LMVECs in Transwell ${ }^{\circledR}$ inserts on PTMC and PET membranes is then presented, characterised and compared to monocultures on similar membranes.

Chapter 7 describes a novel combinatorial method of EIPS and micromoulding to fabricate porous, permeable and morphologically stable PTMC membranes with microwells that resemble alveoli, similar to those in Figure 2. The fabrication method is described, followed by characterisation of the membranes. A co-culture of Calu-3 cells and LMVECs in Transwell@ inserts on these membranes and PET membranes is then presented and characterised. Besides, preliminary results are shown of the implementation of the co-culture on the PTMC membranes with microwells in a commercial OOC-platform.

Chapter 8 presents the main conclusions of the thesis, reflects on the thesis as a whole and discusses potential approaches for future research to optimise, characterise and adapt the presented membranes for further application in biomimetic lung epithelial-endothelial models.

\section{References}

1. Lumen Learning. Microbiology. Anatomy and Normal Microbiota of the Respiratory Tract. Available online: https://courses.lumenlearning.com/microbiology/chapter/anatomy-and-normalmicrobiota-of-the-respiratory-tract/ (accessed on 16 August 2021). Original version from National Cancer Institute.

2. World Health Organization. The Top 10 Causes of Death. Available online: https:/www.who.int/news-room/fact-sheets/detail/the-top-10-causes-of-death (accessed on 12 August 2021).

3. Chapter 2 of this thesis.

4. Lee, J.H.; Lee, S.J.; Khang, G.; Lee, H.B. Interaction of fibroblasts on polycarbonate membrane surfaces with different micropore sizes and hydrophilicity. J Biomater. Sci. Polym. Ed. 1999, 10(3), 283-294. DOI: 10.1163/156856299X00351.

5. Gotoh, K.; Yasukawa, A.; Taniguchi, K. Water contact angles on poly(ethylene terephthalate) film exposed to atmospheric pressure plasma. J Adhes. Sci. Technol. 2011, 25(1-3), 307-322. DOI: 10.1163/016942410X511114. 


\section{- Chapter 1 -}

General introduction

6. Lai, Y.S.; Chen, W.C.; Huang, C.H.; Cheng, C.K.; Chan, K.K.; Chang, T.K. The effect of graft strength on knee laxity and graft in-situ forces after posterior cruciate ligament reconstruction. PLoS One. 2015, 10(5). DOI: 10.1371/journal.pone.0127293.

7. Bat, E.; Zhang, Z.; Feijen, J.; Grijpma, D.W.; Poot, A.A. Biodegradable elastomers for biomedical applications and regenerative medicine. Regen. Med. 2014, 9(3), 385-398. DOI: 10.2217/RME.14.4.

8. Terzini, M.; Bignardi, C.; Castagnoli, C.; Cambieri, I.; Zanetti, E.M.; Audenino, A.L. Ex Vivo Dermis Mechanical Behavior in Relation to Decellularization Treatment Length. Open Biomed. Eng. J. 2016, 10(1), 34-42. DOI: 10.2174/1874120701610010034.

9. Butcher, D.T.; Alliston, T.; Weaver, V.M. A tense situation: forcing tumour progression. Nat. Rev. Cancer. 2009, 9(2), 108-122. DOI: 10.1038/nrc2544.

10. Huh, D.; Matthews, B.D.; Mammoto, A.; Montoya-Zavala, M.; Hsin, H.Y.; Ingber, D.E. Reconstituting organ-level lung functions on a chip. Science. 2010, 328(5986), 1662-1668. DOI: 10.1126/science.1188302.

11. Huh, D.; Kim, H.J.; Fraser, J.P.; Shea, D.E.; Khan, M.; Bahinski, A.; Hamilton, G.A.; Ingber, D.E. Microfabrication of human organs-on-chips. Nat. Protoc. 2013, 8(11), 2135-2157. DOI: 10.1038/nprot.2013.137.

12. Huh, D.; Hamilton, G.A.; Ingber, D.E. From 3D cell culture to organs-on-chips. Trends Cell Biol. 2011, 21(12), 745-754. DOI: 10.1016/j.tcb.2011.09.005.

13. Huh, D.; Leslie, D.C.; Matthews, B.D.; Fraser, J.P.; Jurek, S.; Hamilton, G.A.; Thorneloe, K.S.; McAlexander, M.A.; Ingber, D.E. A human disease model of drug toxicity-induced pulmonary edema in a lung-on-a-chip microdevice. Sci. Transl. Med. 2012, 4(159). DOI: 10.1126/scitranslmed.3004249.

14. Huh, D.; Torisawa, Y.S.; Hamilton, G.A.; Kim, H.J.; Ingber, D.E. Microengineered physiological biomimicry: organs-on-chips. Lab Chip. 2012, 12(12), 2156-2164. DOI: 10.1039/c2lc40089h.

15. Chen, Y.F.; Chan, H.N.; Michael, S.A.; Shen, Y.S.; Chen, Y.; Tian, Q.; Huang, L.; $\mathrm{Wu}, \mathrm{H}$. A microfluidic circulatory system integrated with capillary-assisted pressure sensors. Lab Chip. 2017, 17(4), 653-662. DOI: 10.1039/c6lc01427e.

16. Di Caprio, G.; Stokes, C.; Higgins, J.M.; Schonbrun, E. Single-cell measurement of red blood cell oxygen affinity. Proc. Natl. Acad. Sci. USA. 2015, 112(32), 99849989. DOI: 10.1073/pnas.1509252112.

17. Ochsner, M.; Dusseiller, M.R.; Grandin, H.M.; Luna-Morris, S.; Textor, M.; Vogel, V.; Smith, M.L. Micro-well arrays for 3D shape control and high resolution analysis of single cells. Lab Chip. 2007, 7(8), 1074-1077. DOI: 10.1039/b704449f.

18. Toepke, M.W.; Beebe, D.J. PDMS absorption of small molecules and consequences in microfluidic applications. Lab chip. 2006, 6(12), 1484-1486. DOI: 10.1039/b612140c.

19. Van Meer, B.J.; de Vries, H.; Firth, K.; van Weerd, J.; Tertoolen, L.; Karperien, H.; Jonkheijm, P.; Denning, C.; IJzerman, A.P.; Mummery, C.L. Small molecule absorption by PDMS in the context of drug response bioassays. Biochem. Bioph. Res. Co. 2017, 482(2), 323-328. DOI: 10.1016/j.bbrc.2016.11.062 


\section{- Chapter 1 -}

General introduction

20. Soscia, D.A.; Sequeira, S.J.; Schramm, R.A.; Jayarathanam, K.; Cantara, S.I.; Larsen, M.; Castracane, J. Salivary gland cell differentiation and organization on micropatterned PLGA nanofiber craters. Biomaterials. 2013, 34(28), 6773-6784. DOI: 10.1016/j.biomaterials.2013.05.061.

21. Pêgo, A.P. Preparation and in vivo performance of degradable two-ply nerve guides based on (co)polymers of trimethylene carbonate and $\varepsilon$-caprolactone. Ph.D. thesis, University of Twente, Enschede, The Netherlands, November 2002.

22. Pêgo, A.P.; Siebum, B.; Van Luyn, M.J.; Gallego y Van Seijen, X.J.; Poot, A.A.; Grijpma, D.W.; Feijen, J. Preparation of degradable porous structures based on 1,3-trimethylene carbonate and D,L-lactide (co)polymers for heart tissue engineering. Tissue Eng. 2003, 9(5), 981-994. DOI: 10.1089/107632703322495628.

23. Papenburg, B.J.; Schuller-Ravoo, S.; Bolhuis-Versteeg, L.A.M.; Hartsuiker, L.; Grijpma, D.W.; Feijen, J.; Wessling, M.; Stamatialis, D. Designing porosity and topography of poly(1,3-trimethylene carbonate) scaffolds. Acta. Biomater. 2009, 5(9), 3281-3294. DOI: 10.1016/j.actbio.2009.05.017.

24. Schüller-Ravoo, S.; Papenburg, B.; Stamatialis, D.; Feijen, J.; Grijpma, D. Microstructured Photo-Crosslinked Poly(Trimethylene Carbonate) for Use in Soft Lithography Applications: A Biodegradable Alternative for Poly(Dimethylsiloxane). ChemPhysChem. 2018, 19(16), 2085-2092. DOI: 10.1002/cphc.201701308.

25. Bat, E.; Feijen, J.; Grijpma, D.W. Biodegradable elastomeric networks: highly efficient cross-linking of poly(trimethylene carbonate) by gamma irradiation in the presence of pentaerythritol triacrylate. Biomacromolecules. 2010, 11(10), 2692-2699. DOI: 10.1021/bm1007234.

26. Bat, E.; Kothman, B.H.M.; Higuera, G.A.; van Blitterswijk, C.A.; Feijen, J.; Grijpma, D.W. Ultraviolet light crosslinking of poly(trimethylene carbonate) for elastomeric tissue engineering scaffolds. Biomaterials. 2010, 31(33), 8696-8705. DOI: 10.1016/j.biomaterials.2010.07.102.

27. Allijn, I.; Ribeiro, M.; Poot, A.A.; Passier, R.; Stamatialis, D. Membranes for Modelling Cardiac Tissue Stiffness In Vitro Based on Poly(trimethylene carbonate) and Poly(ethylene glycol) Polymers. Membranes. 2020, 10(10), E274. DOI: 10.3390/membranes10100274.

28. Schüller-Ravoo, S.; Feijen, J.; Grijpma, D.W. Flexible, elastic and tear-resistant networks prepared by photo-crosslinking poly(trimethylene carbonate) macromers. Acta. Biomater. 2012, 8(10), 3576-3585. DOI: 10.1016/j.actbio.2012.06.004.

29. Pêgo, A.P.; Poot, A.A.; Grijpma, D.W.; Feijen, J. Copolymers of trimethylene carbonate and epsilon-caprolactone for porous nerve guides: synthesis and properties. Biomater. Sci. Polym. Ed. 2001, 12(1), 35-53. DOI: $10.1163 / 156856201744434$.

30. Zant, E.; Bosman, M.J.; Grijpma, D.W. Combinatorial synthesis of photocrosslinked biodegradable networks. J. Appl. Biomater. Funct. Mater. 2012, 10(3), 197-202. DOI: 10.5301/JABFM.2012.10344.

31. Bat, E.; van Kooten, T.G.; Feijen, J.; Grijpma, D.W. Resorbable elastomeric networks prepared by photocrosslinking of high-molecular-weight 
poly(trimethylene carbonate) with photoinitiators and poly(trimethylene carbonate) macromers as crosslinking aids. Acta. Biomater. 2011, 7(5), 19391948. DOI: 10.1016/j.actbio.2011.01.010.

32. Zant, E.; Blokzijl, M.; Grijpma, D.W. A Combinatorial Photocrosslinking Method for the Preparation of Porous Structures with Widely Differing Properties. Macromolecular rapid communications. 2015, 36(21), 1902-1909. DOI: 10.1002/marc.201500229. 
- Chapter 1 -

General introduction

$-14-$ 


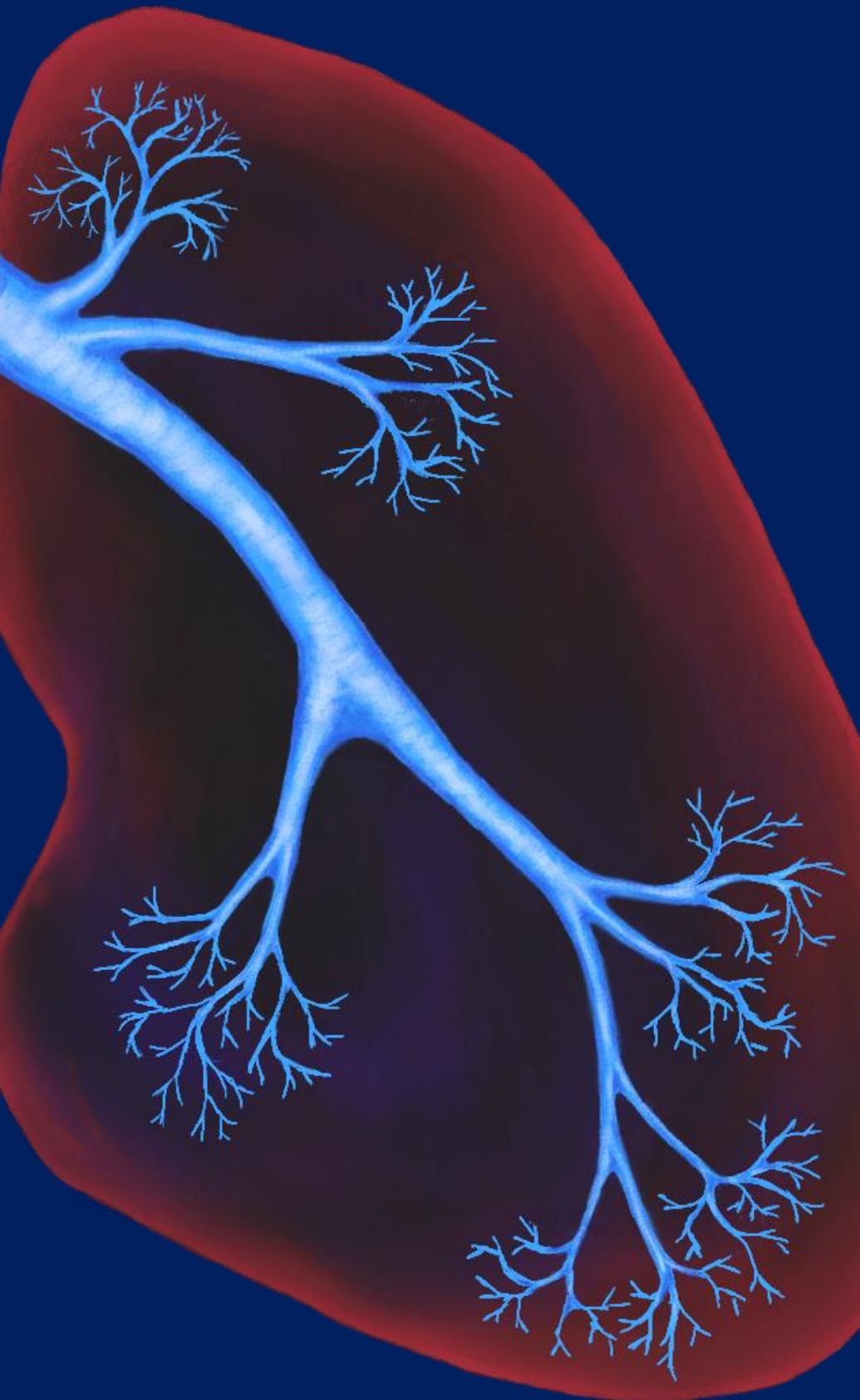




\section{- Chapter 2 -}

\section{Flat and microstructured polymeric membranes in organs-on-chips}

Thijs Pasman ${ }^{1}$, Dirk W. Grijpma ${ }^{1,2}$, Dimitrios Stamatialis ${ }^{1}$ and André A. Poot ${ }^{1}$

1. Technical Medical (TechMed) Centre, Department of Biomaterials Science and Technology, Faculty of Science and Technology, University of Twente, 7522 NB Enschede, The Netherlands

2. Department of Biomedical Engineering, Universitair Medisch Centrum Groningen, 9700 AB Groningen, The Netherlands

This chapter was adapted from: Pasman, T.; Grijpma, D.; Stamatialis, D.; Poot, A. Flat and microstructured polymeric membranes in organs-on-chips. Journal of the Royal Society, Interface. 2018, 15(144), 20180351. DOI: 10.1098/rsif.2018.0351. 
Flat and microstructured polymeric membranes in organs-on-chips

\section{Abstract}

In recent years, organs-on-chips (OOCs) have been developed to meet the desire for more realistic in vitro cell culture models. These systems introduce microfluidics, mechanical stretch and other physiological stimuli to in vitro models, thereby significantly enhancing their descriptive power. In most OOCs, porous polymeric membranes are used as substrates for cell culture. The polymeric material, morphology and shape of these membranes are often suboptimal, despite their importance for achieving ideal cell functionality such as cell-cell interaction and differentiation. The currently used membranes are flat and thus do not account for the shape and surface morphology of a tissue. Moreover, the polymers used for fabrication of these membranes often lack relevant characteristics, such as mechanical properties matching the tissue to be developed and/or cytocompatibility. Recently, innovative techniques have been reported for fabrication of porous membranes with suitable porosity, shape and surface morphology matching the requirements of OOCs. In this paper, we review the state of the art for developing these membranes and discuss their application in OOCs.

Keywords: membranes; biomaterials; polymers; microfabrication; organs-on-chips

\section{Introduction: the need for organs-on-chips}

The development of new drugs is becoming more difficult, timeconsuming and costly. Despite all the trials done before approval of a drug, reactions of patients to the drug can differ greatly. In fact, individuals can even react adversely to a drug. These responses do not always become apparent in the first stages of clinical trials. Participants are often selected on characteristics such as sex, age or ethnicity and thus do not represent the entire population. Also, due to ethical reasons, the effects of drugs on children are hardly investigated. As a result of these limitations, clinical trials often do not show the entire spectrum of responses to a drug. Moreover, many drugs fail during clinical trials, despite showing efficacy during preclinical trials. The notion that we 
Flat and microstructured polymeric membranes in organs-on-chips

cannot fully rely on animal models is growing. Despite a considerable resemblance in both genetics and physiology between animal models and humans, animal models often do not accurately predict how drugs will perform in humans.

In vitro models have the advantage that they often use only human cells. However, they lack the physiology of a tissue, and thus cell behaviour may differ from the in vivo situation. Transwell@ inserts consist of small baskets with a porous, permeable membrane underneath which can be placed in a well plate. This way, the membrane is suspended above the bottom of the well (Figure 1A). Lung cells, for example, can be placed on the membrane and exposed to air (Figure 1A), while receiving nutrients through the membrane. This already allows more realistic cell culturing than traditional systems. However, the cells do not experience stimuli such as airflow or mechanical stretch.

A

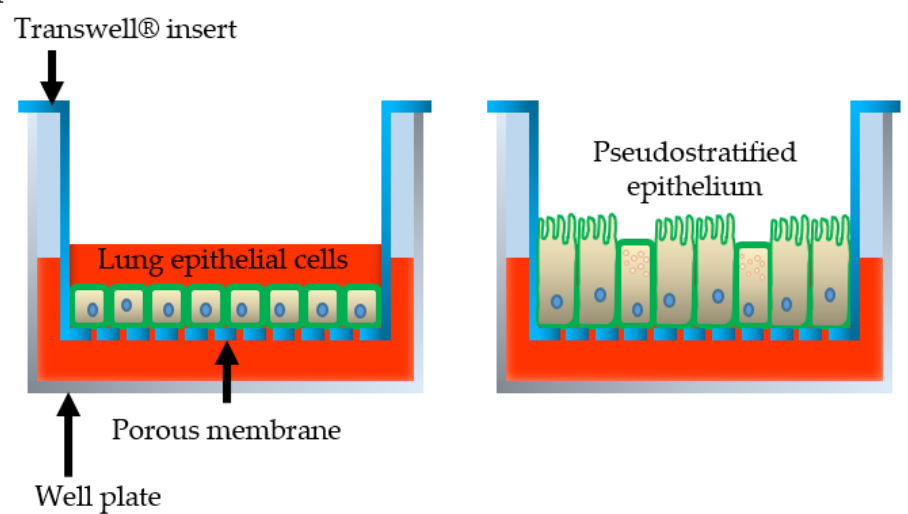

Submerged culture

Air-liquid interface culture

B

Organ compartment

Blood vessel compartment

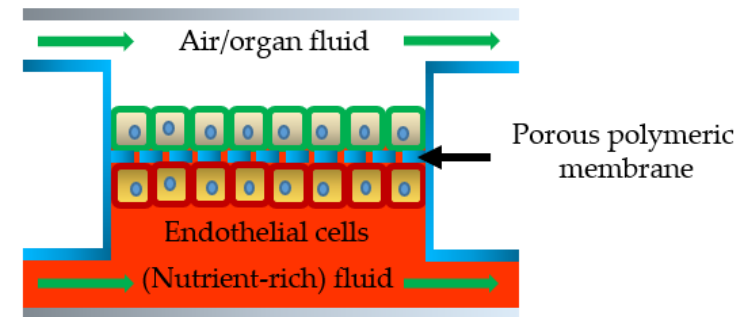


- Chapter 2 -

Flat and microstructured polymeric membranes in organs-on-chips

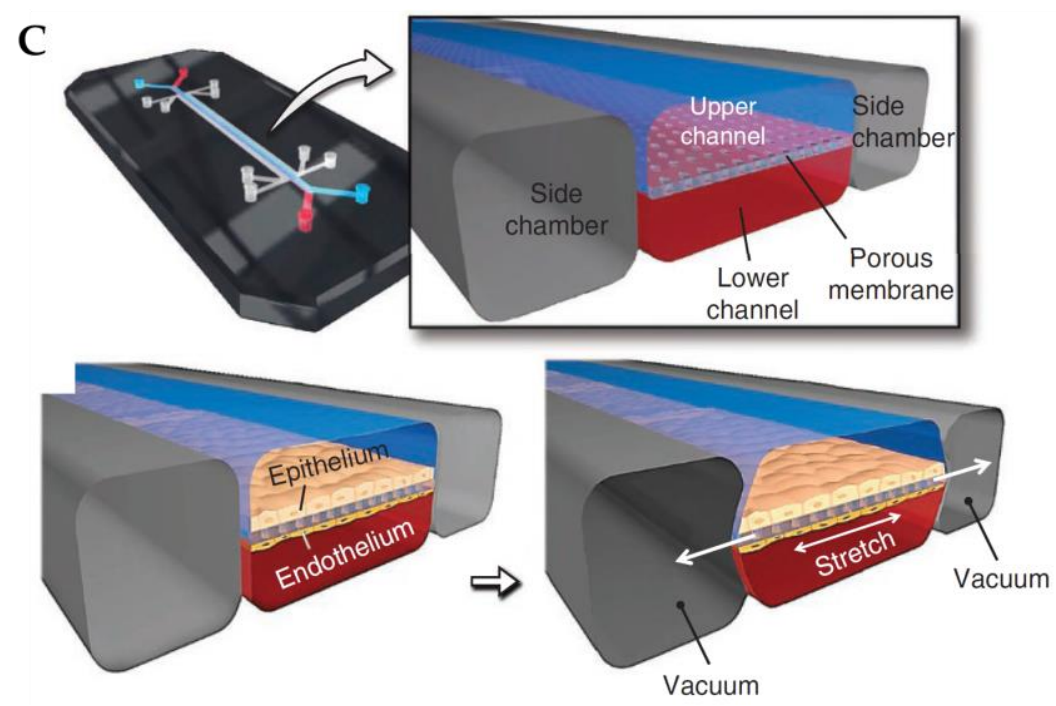

Figure 1. Schematic representation of lung cell culture systems. (A) Lung epithelial cells are cultured in a conventional manner on a porous poly(bisphenol-A carbonate) (PC) or poly(ethylene terephthalate) (PET) membrane using a commercially available Transwell ${ }^{\circledR}$ system. Initially, cell culture medium is provided both on top of the cells and below the insert, i.e. submerged culture. Subsequently, the medium on top of the cells is removed to expose the cells to air, i.e. air-liquid interface culture. This causes the cells to transition to a pseudostratified epithelium. Nutrients from the cell culture medium pass through the membrane. (B) OOCs generally comprise two compartments that are connected via a porous, polymeric membrane. The blood vessel compartment contains a flowing fluid which substitutes the blood in the particular organ, often containing the nutrients and other factors which the cells need, such as cell culture medium. Endothelial cells can be cultured on the membrane to represent the blood vessel wall. The organ compartment holds cells of the organ of interest, for example liver, kidney or lung epithelial cells which are grown on the other side of the membrane. The compartment is usually filled with a medium mimicking the fluid in the organ or, for example, air, in the case of the lung or skin. In most OOCs, this medium flows to mimic the flow of air or liquid in the organ. (C) A lung-on-a-chip consisting of a microfluidic device with three channels. The middle channel contains two compartments, separated by a porous poly(dimethyl siloxane) (PDMS) membrane. Lung epithelial cells are cultured on the top side, while endothelial cells are cultured on the bottom side of the membrane. Air and cell culture medium flow through the top and bottom 
Flat and microstructured polymeric membranes in organs-on-chips

compartment, respectively. A vacuum can be applied in the adjacent two channels which provide mechanical stretch to the membrane and cells. Adapted from Huh et al. [1].

In vitro models that better predict drug responses and can account for individual differences would address many of the issues in drug development. Because of the need of such in vitro models, organs-onchips (OOCs) have been developed [1-48]. These are small microfluidic devices that mimic a particular organ by introducing organ-like features such as fluid flow and mechanical stress (Figure 1B,C). Cell lines are used in most OOCs, but the need for the development of personalised therapies leads to the desire of using human-induced pluripotent stem cells (hiPSCs). With iPSC technology, differentiated cells can be dedifferentiated to an embryonic stem cell-like state, after which they can be redifferentiated to any cell type. The development of OOCs and iPSC technology could result in powerful tools for developing personalised therapies. Moreover, iPSC technology could also provide an opportunity to test drugs on cells derived from children or other parts of the population which are generally not investigated. By giving a more accurate prediction of drug responses in humans, fewer drugs would fail in clinical trials, thus decreasing overall development costs and time. Moreover, because of their small size, OOCs only need small amounts of resources and thus are not expensive. OOCs could also lead to a decrease in animals needed for research, reducing the ethical difficulties related to drug development. Ultimately, they can provide patients with more effective drugs faster, cheaper and with a lower ethical impact.

Although each type of OOC is different in order to mimic a specific organ properly, many of their characteristics are the same (Figure 1B). They often consist of a 'blood vessel compartment' containing endothelial cells and an 'organ compartment' containing cells of the tissue or organ of interest. Between the two compartments, generally, a porous polymeric membrane is applied for achieving cell adhesion and cell separation as well as cell communication between the two compartments. In this 
Flat and microstructured polymeric membranes in organs-on-chips

review, membranes are defined as interfaces between two phases. Membranes have a selective permeability, based on the membrane structure among other factors. Therefore, a distinction can be made between porous membranes which contain microscopic pores and nonporous membranes which only contain molecular pores, i.e. intermolecular spaces through which gases can diffuse. This is based on the definition of a membrane given by Mulder [49].

In OOCs, the different cell types are grown on opposite sides of the membrane. The blood vessel compartment can be perfused with aqueous solutions of nutrients, such as cell culture medium or a blood substitute (Figure 1B,C). The nutrients will need to pass the endothelial cells and the porous membrane to supply the cells of interest that are cultured on the other side of the membrane. Similar to the blood vessel compartment, the organ compartment can often be perfused, for example, with a nutrientrich fluid in a gut-on-a-chip $[8,9,18,19,29,39,50,51]$ (Figure 1B) or ventilated with air as would be appropriate for a lung-on-a-chip $[11,12,31,36,42]$ (Figure 1B,C). In addition, applying mechanical forces to the cells by stretching the cell-covered membranes can simulate the peristalsis in the intestines or the respiratory movement in the lungs $[1,10-13,18,19,42]$.

Despite the drastic advancement in OOC technologies in general (better sensors, pumps and microfluidics) with a rapidly expanding library of OOCs of different organs or redesigns of already existing ones, the advancements in the development of appropriate porous, polymeric membranes are very slow. Only a handful of materials are applied for membranes in OOCs (Table 1). These membranes and the materials they are made of are very relevant in these models because they provide structural support for the different cells while also allowing nutrient transport to the separate compartments. It is also known that cells react to environmental cues like chemistry, stiffness, topography and curvature of their substrate. However, important aspects of the membranes used in OOCs and other in vitro models, such as their material and shape, are often neglected. 
Flat and microstructured polymeric membranes in organs-on-chips

Table 1. Polymers used for fabrication of porous membranes in OOCs and microfluidic systems.

\begin{tabular}{ll}
\hline Polymer & Application \\
\hline poly(dimethyl siloxane) (PDMS) & artery [6] \\
\hline erythrocyte oxygenation [7] \\
\hline gut [1,18,19] \\
\hline heart [40] \\
\hline kidney [13] \\
\hline liver [1,3,13,38] \\
\hline lung [1,11,13,42] \\
\hline multi-organ-on-chip (MOC) of liver and \\
heart [47] \\
\hline new membranes for OOCs [52] \\
\hline blood-brain-barrier (BBB) [4,43,44] \\
\hline bonding of membrane to chip [51] \\
\hline colon and breast cancer [27] \\
\hline gut [8,9] \\
\hline gut, liver and brain cancer [16] \\
\hline MOC of liver and intestine [29] \\
\hline MOC of liver and skin [29] \\
\hline multiple-layered cultures of fibroblasts, \\
endothelial and mesenchymal cells [41]
\end{tabular}


- Chapter 2 -

Flat and microstructured polymeric membranes in organs-on-chips

Others

poly(amide)

gut [50]

parylene $\mathrm{C}$

liver [46]

(dichloro[2,2]paracyclophane)

poly(tetrafluoroethylene) (PTFE) liver (hepatic sinusoid) [14]

poly(urethane acrylate) (PUA) new membranes for OOCs [52]

poly(ethylene glycol)-diacrylate (PEG- new membranes for OOCs [52]

DA)

In fact, several OOCs simply use membranes found in commercial inserts $[2,15,21,26,29,39,43,48,53]$ or filter membranes $[4,16,23,25,27,41,43,44,51]$. Many are made of polymers that have suboptimal cytocompatibility and often do not have suitable mechanical properties matching the tissue to be developed. To accurately simulate in vivo situations, the membranes used should stimulate natural behaviour of cells and should be made with a surface structure that mimics the architecture of the tissue.

This review focuses on the characteristics and fabrication of porous polymeric membranes used in OOCs, as well as the importance of their characteristics in relation to cell behaviour. The main polymers that have been used for membranes are presented, and the techniques used to introduce porosity in these membranes are discussed. We also highlight other factors affecting cell behaviour, mainly the topography of the surface the cells are growing on, and we include methods to manufacture porous polymeric membranes that more realistically mimic in vivo microenvironments. These membranes could further improve OOCs.

\section{Material properties affecting cells}

A very important requirement for producing optimal OOCs is the application of a polymeric membrane which supports the cells and functions as an equivalent of the extracellular matrix (ECM) or more specifically the cell basement membrane found in natural tissue. In this section, we will first briefly discuss important characteristics of the ECM and then focus on important polymeric membrane characteristics tailored to mimic the ECM. 
Flat and microstructured polymeric membranes in organs-on-chips

\subsection{Need for mimicking the extracellular matrix}

Both the ECM and the basement membrane comprise a complex arrangement of components, mostly proteins such as collagens, fibronectin and vitronectin. These proteins contain amino acid sequences like arginine-glycine-aspartic acid (RGD), which are potent sites for cell adhesion [54,55]. Cells interact with these sites via integrins, transmembrane proteins which can also link to the actin cytoskeleton of the cells. Through the connection between the ECM, cell membrane and cytoskeleton, the ECM can affect the cells both mechanically and biochemically.

These interactions between cells and cell-adhesive proteins can also play a role in the case of cell substrates other than the ECM. The adsorption of cell-adhesive proteins containing RGD sequences onto a surface can stimulate cell attachment to that surface [54,56,57]. Moreover, the conformation of the adsorbed proteins is very important [54]. If the RGD sequences are denatured or not accessible to the cells, adherence may be compromised.

\subsection{Surface roughness}

The surface roughness of a material can significantly influence cell behaviour [57-60]. Lampin et al. [57] sandblasted the surface of poly(methyl methacrylate) (PMMA) films with alumina grains to alter the surface roughness. Adhesion and migration of vascular and corneal cells increased with higher surface roughness. Proliferation was, however, not affected. They suggested that the increased cell adhesion on films with a higher roughness was due to more adsorbed proteins, such as fibronectin and collagen I and III, on the surface of the films. Dowling et al. [58] cultured MG63 osteosarcoma cells on poly(styrene) with varying roughness. Adhesion of the cells was higher on rough films than on smooth films. Conversely, cell spreading decreased as surface roughness increased. Moreover, MG63 cells showed a decreased cell spreading and an increased integrin expression on titanium discs with rough surfaces compared to discs with smooth surfaces [60]. Kunzler et al. [59] showed that different cell types could be affected very differently by the surface. 
Flat and microstructured polymeric membranes in organs-on-chips

Rat calvarial osteoblasts (RCOs) and human gingival fibroblasts (HGFs) were cultured on aluminium sheets with a roughness gradient. Spreading of both cell types was reduced on rough surfaces. However, as roughness increased, the proliferation of RCOs increased while that of HGFs decreased.

\subsection{Hydrophilicity}

Hydrophilicity of a material is a strong determinant of protein adsorption and thus cell adhesion [56,57]. Wala et al. [61] prepared PDMS films with different hydrophilicity (by either oxygen plasma or piranha treatment) and reported that cell attachment, spreading and growth of 3T3 fibroblasts and $\mathrm{HaCaT}$ keratinocytes increased with increasing hydrophilicity of the films. Others found that moderate hydrophilicity, i.e. water contact angles of approximately $55-65^{\circ}$, often yields the best cell response [56,62]. Lee et al. [56] increased the hydrophilicity of poly(ethylene) films by surface oxidation. Moderate hydrophilicity led to the highest cell adhesion, spreading and growth, while cells performed worse on films with low or very high hydrophilicity. Adsorption of serum proteins also showed an optimum on films with moderate hydrophilicity. The adsorbed proteins were probably the main cause of the increased cell response. Premnath et al. [62] used a laser to introduce nano-patterns in silicon wafers which made the surface more hydrophilic. Again, moderate hydrophilicity increased adhesion of HeLa cells as well as adsorption of cell-adhesive proteins, whereas high surface hydrophilicity decreased adsorption of the proteins and cell adhesion. They also suggested that the superior cell-adhesive properties of moderately hydrophilic surfaces are due to the higher adsorption of cell-adhesive proteins, when compared with hydrophobic or highly hydrophilic surfaces.

\subsection{Mechanical properties}

It is suggested that initial cell adhesion and spreading are regulated by material surface properties, such as hydrophilicity and roughness, while properties such as the stiffness of a cell substrate influence later stages of cell growth [61]. For example, Wala et al. [61] reported that 3 T3 fibroblasts 
Flat and microstructured polymeric membranes in organs-on-chips

proliferated more on stiffer PDMS films (Young's modulus of 2.6-3.2 $\mathrm{MPa}$ ), while HaCaT keratinocytes showed higher proliferation on softer PDMS films (Young's modulus of 0.6-1 MPa). Wen et al. [63] cultured adipose stem cells (ASCs) on poly(acrylamide) (PAA) hydrogels with varying stiffness. Soft hydrogels directed cells towards adipogenic differentiation, while stiff hydrogels led to osteogenic differentiation. Engler et al. [64] differentiated mesenchymal stem cells (MSCs) on collagen I-coated PAA substrates and showed that the lineage of differentiation depended on the stiffness of the substrate. Substrates with a stiffness corresponding to brain $(0.1-1 \mathrm{kPa})$ and muscle $(8-17 \mathrm{kPa})$ led to neurogenic and myogenic differentiation, respectively, while stiff collagen I substrates $(25-40 \mathrm{kPa})$ led to osteogenic differentiation. When the cell culture medium was changed after one week of culturing on one of these substrates to a differentiation medium for a different lineage, many cells began showing traits of that lineage. However, when the medium was changed after three weeks of culturing, cells were much less plastic. The effect of the stiffness of the substrate was strong enough to lead cells towards a stable commitment for a certain lineage.

Ye et al. [55] looked at the mechanisms involved between substrate stiffness and differentiation by culturing MSCs on poly(ethylene glycol) (PEG) hydrogels with varying stiffness. The hydrophilicity of PEG avoided protein adsorption and cell adhesion, and thus cells could only attach to RGD peptides bound to the surface. Softer hydrogels stimulated adipogenesis, while stiffer hydrogels resulted in osteogenesis. Stiff hydrogels probably led to more focal adhesions and thus more cell tension, inducing osteogenic differentiation, while fewer focal adhesions and less cell tension on softer hydrogels stimulated adipogenesis. Interestingly, Casillo et al. [53] cultured endothelial cells on PDMS films with varying stiffness but did not see an influence of PDMS stiffness on the formation of focal adhesions. It seems that the effect of material stiffness depends on the cell type applied. 
Flat and microstructured polymeric membranes in organs-on-chips

\subsection{Porosity}

Porosity is an important characteristic of the membranes in OOCs for achieving cell communication between the chip compartments as well as for achieving oxygen and nutrient transport to the cells. Casillo et al. [53] grew endothelial cells on non-porous and porous $\mathrm{SiO}_{2}$ membranes. The porous membranes had different pore sizes and corresponding pore spacings. Cells showed fewer focal adhesions and less fibronectin fibrillogenesis on porous membranes, and overall cells showed less interaction with the porous membranes. This effect was stronger on membranes with smaller pores, probably because the spacing between the pores, and thus the amount of continuous space for the cells to adhere was smaller. However, cell-cell interactions in the form of tight junctions measured by ZO-1 activity showed an opposite trend. Porosity, especially with small pore spacing, could thus limit cell adhesion, but enhance barrier function of cells. They concluded that weaker cell-substrate interaction might lead to stronger cell-cell interaction. Wen et al. [63], however, found no influence of the porosity on the differentiation of ASCs on PAA hydrogels, suggesting that the effect of porosity on cells may depend on multiple factors.

\subsection{Microstructures}

\subsubsection{Microstructures of singular cell size}

Hulsman et al. [65] prepared a cell culture chip from poly(lactic acid) (PLA) that contained a large number of differently designed surface microtopographies. Human MSCs responded differently to the different topographies. Especially the amount of open space between the microstructures was important. Cell and nucleus morphology was rounded when cells had enough space to spread, while they had an abnormal shape when they were forced to compact when the spacing between structures was small. Moreover, cells aligned in between structures with a specific spacing. The best alignment was found at spacings of 2.5-14.1 $\mu \mathrm{m}$, while smaller spacings did not lead to proper alignment. These responses could have major implications for cell behaviour. Reimer et al. [66] confirmed this by using a similar chip 
Flat and microstructured polymeric membranes in organs-on-chips

prepared from PLA. They found that some topographies could stimulate Oct4 expression and proliferation of hiPSCs, while other topographies decreased it. In general, smaller feature sizes and higher densities of the shapes on the PLA surface stimulated pluripotency of the cells. These results imply that the topography of the surface can potentially trigger a cell to differentiate or remain in a proliferative state. The effect could be the result of mechanosensory pathways, which are activated by specific topographies.

Ochsner et al. [67] cultured human umbilical vein endothelial cells (HUVECs) on PDMS films which contained fibronectin-coated microwells of different shapes and sizes to investigate the effect of the microwells on single-cell behaviour. The space between the microwells was coated with poly(L-lysine)-graft-poly(ethylene glycol) to avoid cell growth outside of the microwells. Smaller microwells confined the cell shape. This confinement led to a more compact cytoskeleton, while the cytoskeleton of cells grown in larger microwells did spread. Cells in smaller microwells often showed actin stress fibres parallel to the length of the microwell. Geometry can also result in survival or death of cells as shown by Chen et al. [68]. They grew human capillary endothelial cells on culture dishes coated with fibronectin (FN) patterns of various shapes and sizes, ranging from the size of single cells to small clusters. Cells preferred growing on the coated areas instead of the culture dish. Better cell spreading, either by bigger patches of fibronectin or spacing between patches, which allowed spreading from one patch to the other, decreased apoptosis, while survival and growth were promoted. The results were independent of the contact area between cells and the ECM, and thus cell shape seemed to be critical between survival and death.

\subsubsection{Microstructures larger than a cell}

The studies mentioned above mainly looked at surface topographies in the same size range as a single cell. Chen et al. [68] showed that larger topographies involving multiple cells also have significant influence on clusters of cells. Lee et al. cultured a murine cancer cell line on hydrazinepatterned PAA gels to which ECM proteins were bound in different 
Flat and microstructured polymeric membranes in organs-on-chips

geometries. In general, patterned gels raised tumorigenic and stem-celllike behaviour, especially at the edges of the tumour. Their results pointed towards a potential role of geometry in the occurrence of metastasis [69]. Similar results were seen with bovine pulmonary artery endothelial cells grown on glass surfaces that contained patches coated with FN [70]. Cells covered the areas coated with FN. Proliferation of the cells was higher at the edges of the areas than at the centre. The geometry of the coated area, and thus the cell colony, influenced the proliferation. A higher amount of stress at the edges of the areas compared to the centre was probably the cause of the increased proliferation at the edges. Human MSCs have also been shown to change their differentiation depending on the substrate shape [71]. The MSCs were grown on FN-patterned glass surfaces. In general, the cells seemed to have a preference for osteogenic differentiation on shapes with sharper edges, while adipogenic differentiation was more common on squares as well as shapes with rounded corners.

Cells do not only respond to topographies as mentioned above. Large, three-dimensional microstructures with sizes of up to several hundreds of micrometres in both width and height can also direct cell behaviour. Papenburg et al. [72,73] showed the influence of microstructures on the orientation of cells growing on a microstructured membrane. Moreover, there is evidence that cells that are grown in microstructures also arrange their ECM in a more organised manner [74]. The mechanical strength of engineered tissues, consisting of cells and their ECM, improved when grown on microstructured films instead of flat films [74]. Soscia et al. [75] fabricated PDMS films containing microwells coated with poly(lactic-coglycolic acid) (PLGA) nanofibres. They found that increasing the curvature of the microwells caused an increase of polarity in salivary gland cell lines and a higher expression of the tight junction protein occludin at the apical side. Also, the cells showed signs of differentiation. The magnitude of the curvature affected the strength of the response. 
Flat and microstructured polymeric membranes in organs-on-chips

Esch et al. [76] grew HUVECs on PDMS films with square and semicircular microfluidic channels, both under static conditions and under flow. Cell-cell interactions were similar across the different culturing conditions as shown by expression of VE-cadherin. However, vinculin, a measure of focal adhesions, was less present in square channels than in semicircular ones, suggesting that semicircular channels led to a better cell-surface communication. Others have shown that stress fibres were primarily present at the edges of microstructures and that proliferation was also high at those locations [70,77], further elucidating the influence of the surface morphology on cells. Conversely, Hebeiss et al. [78] showed that endothelial cells produced fewer stress fibres and focal adhesions when cultured on semicircular porous channels with a $200 \mu \mathrm{m}$ diameter when compared with flat membranes. Yamashita et al. [79] cultured human aortic endothelial cells and human aortic smooth muscle cells on concave surfaces. Retaining the concave shape of the cell layer became more difficult when contraction of the cells was stimulated by the addition of TGF- $\beta$, and thus cells detached. Cell detachment also increased when the concave shape of the substrate was larger. Additionally, Broaders et al. [80] showed that detachment of cells on PDMS substrates with concave channels is dependent on the cell type. For example, nearly all HUVECs detached from the channels, while MadinDarby Canine Kidney-Ras cells remained attached to the substrate. Detachment of cells was susceptible to factors which affect contractility of the cells such as blebbistatin, which decreased detachment. Interestingly, larger flat areas between channels increased detachment. They suggested that the flat areas changed the tension in the cells residing in the channels. The machinery involved in cell-substrate and cell-cell interactions as well as cell contraction probably influences each other, causing cells to react to topography with which they are not directly in contact. Channel-like microstructures could potentially mimic other tissues such as small airways, kidney tubules or intestines and thus serve as a better in vitro model as it is clear that surface geometry influences cell behaviour. However, there is still little understanding of the exact mechanisms. 
Flat and microstructured polymeric membranes in organs-on-chips

\section{Polymers used in the preparation of membranes for organs-on-chips}

Although several polymers have been used to fabricate porous membranes for OOCs and microfluidic systems, only a handful of polymers, i.e. PDMS, poly(carbonate) (PC), poly(ethylene terephthalate) (PET), PLA and poly ( $\varepsilon$-caprolactone) (PCL), were used in the majority of the studies (Table 1). This is often driven by practical reasons rather than properties of the polymer related to the application. For example, PDMS membranes are rather easy to make and they are transparent (and thus easily applied with microscopy) and flexible (and as a result can be easily handled). PC is very often used in Transwell@ cell culture inserts, and therefore reference data are widely available. Using PC in OOCs thus seemed to be a natural way of transferring knowledge from the standard cell culture system. Besides, the notion that the interaction of the membrane with the cells can be tailored by application of surface treatments and/or bioactive coatings on the above widely used materials seems to limit the application of a broader range of materials. In the last few years, it has become more and more evident that also other characteristics of the membrane, such as the mechanical properties, have to be taken into account $[55,61,63]$. For example, in the case of membranes that need to be stretched, an elastic polymer is preferable because it has to withstand deformations for many cycles. Whether a polymer degrades and how it degrades is another important consideration as this would determine possible changes in the membrane properties in time and whether cytotoxicity would occur.

\subsection{Poly(dimethyl siloxane)}

PDMS (Table 2) is often used in OOCs (Tables 1 and 3) [1,3, 5-8,10$13,15,16,18,19,23,25,26,28,29,33,35,36,38,42,47]$. In fact, it is the state-ofthe-art material in OOC technology, used for both the membrane for cell adhesion and for fabrication of the chip itself. It is a hydrophobic polymer which is flexible and transparent, which makes it a desirable material to 
Flat and microstructured polymeric membranes in organs-on-chips

work with for OOCs, as the chips $[1,3,5-8,10-13,15,16,18,19,23,25$, $26,28,29,33,35,36,38,42,47]$ can withstand mechanical forces, and cells are easily visible. PDMS is inert and non-biodegradable. It is, however, known to absorb small molecules and drugs $[87,88]$. This is very relevant because drug studies are an important application for OOCs. One approach to limit the absorption is by coating PDMS, e.g. with a lipidbased coating [88]. In most cases, PDMS needs to be coated with proteins to improve cell adhesion and proliferation. It is also possible to alter the surface chemistry of PDMS to improve cell adhesion [61].

PDMS has a low glass transition temperature $\left(\mathrm{T}_{\mathrm{g}}\right)$ of $-125^{\circ} \mathrm{C}$. To prepare creep-resistant PDMS structures, it is cross-linked. A mixture of PDMS prepolymer and a crosslinker is prepared, cast and afterwards exposed to elevated temperatures or UV light. The mechanical properties of PDMS can be tuned by changing the ratio of PDMS to curing agent $[61,67]$. Membranes with a Young's modulus as low as $4 \mathrm{kPa}$ can be prepared [67], which makes it ideal for cells which prefer a soft substrate, such as cardiomyocytes, which need to contract their substrate. Higher amounts of curing agent can yield PDMS with a modulus of several MPa, suitable for cells which prefer a stiffer substrate. This method of casting and curing is often used together with soft lithography to prepare both PDMS membranes and microfluidic chips. These include OOCs mimicking heart $[6,30,40]$, liver $[1,3,13,38]$, lung $[1,11,13,42]$, a multiorgan-on-chip of liver and heart [47], oxygenation of erythrocytes [7] and vascular networks [35].

Table 2. Chemical structures and properties of commonly used polymers for porous membranes in OOCs. The $\mathrm{T}_{\mathrm{g}}$ is the temperature under which the material behaves like glass and thus is stiff. At temperatures above the $\mathrm{Tg}_{\mathrm{g}}$, polymers are more flexible. The Young's modulus is a measure of stiffness. A high modulus corresponds to high stiffness, while polymers with a low modulus are flexible. The water contact angle is the angle between a water droplet and a polymer film. Polymers with a high water contact angle are hydrophobic, while those with a low contact angle are hydrophilic. The values mentioned are contact angles measured with smooth polymer films as surface roughness changes the contact angle. 
- Chapter 2 -

Flat and microstructured polymeric membranes in organs-on-chips

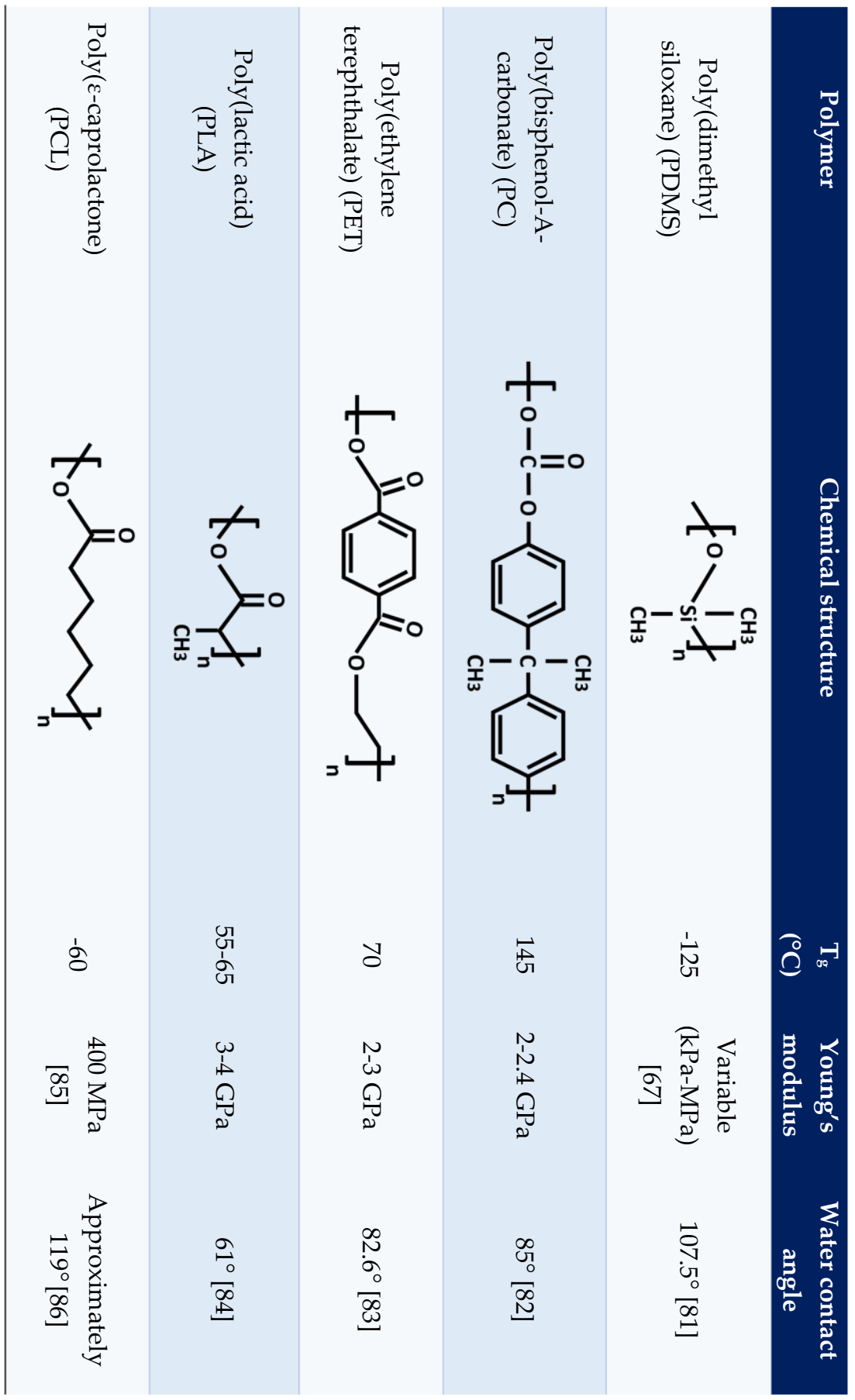


Flat and microstructured polymeric membranes in organs-on-chips

\subsection{Poly(carbonate)}

PC (Table 1) is widely used in OOCs (Tables 1 and 3) $[4,8,9,16,23,27,29,41,43,44,51]$. It is also one of the most commonly used polymers for porous filter membranes and the porous membranes in Transwell@ inserts. In fact, several OOCs implemented PC filter membranes $[4,16,23,25,27,41,43,44,51]$ or PC membranes taken from Transwell@ inserts $[29,43]$. PC is hydrophobic, transparent, inert and nonbiodegradable. For cells to properly adhere and grow on PC, the surface of PC is often altered by protein coating $[4,9,23,44]$ or gas plasma treatment [43] similar to PDMS. PC, however, is a very stiff polymer and has a $\mathrm{T}_{\mathrm{g}}$ of $145^{\circ} \mathrm{C}$ and a Young's modulus of $2-2.4 \mathrm{GPa}$. As a result, PC is not suitable for OOCs which require membrane stretching and for culturing of tissues which need a soft substrate. Nevertheless, PC has been used in many different OOCs, including studies on the blood-brainbarrier (BBB) $[4,43,44]$, cancer [16,25,27] and liver [29]. Surprisingly, however, it has also been used for skin $[23,29]$ and gut $[8,29]$ OOCs, which would ideally require the cells to be mechanically stimulated by a flexible membrane.

\subsection{Poly(ethylene terephthalate)}

PET (Table 2) is another popular polymer for commercial membranes and inserts. Several OOCs and microfluidic cell culture systems included PET membranes (Tables 1 and 3), often PET membranes which were taken directly from inserts $[2,15,21,26,39,48,53]$ or porous filter membranes [28]. PET is transparent, inert and non-biodegradable and also requires treatment to improve cell adhesion, such as plasma treatment. It has a $\mathrm{T}_{\mathrm{g}}$ of $70{ }^{\circ} \mathrm{C}$ and a Young's modulus of approximately 2-3 GPa. Thus, like PC, PET is not suitable for OOCs which require mechanical strain on the cells. It has been used, however, for OOCs mimicking gut [39] and studies on endothelial cells $[2,28]$ as well as fat $[26]$, liver $[17,21,48]$ and kidney [15]. 
Flat and microstructured polymeric membranes in organs-on-chips

3.4. Aliphatic polyesters: poly(lactic acid) and poly(kcaprolactone)

The aliphatic polyesters PLA [33] and PCL [34] (Table 2) have been used in in vitro models mimicking the BBB (Table 1). Both polymers are hydrophobic and biodegradable. This could hold potential for functioning as a temporary membrane, which is later replaced by the ECM of the cells to create an entirely natural cell layer. It has to be taken into account, however, that due to the degradation, the membrane pores might change over time. Moreover, the degradation products of these esters are acidic and thus could affect cells. The $T_{g}$ of PLA and PCL differs significantly at approximately $55-65{ }^{\circ} \mathrm{C}$ and $-60{ }^{\circ} \mathrm{C}$, respectively. As a result, $\mathrm{PCL}$ is in a more deformable state at room or body temperature than PLA. This is accompanied by a Young's modulus of $400 \mathrm{MPa}$ for PCL [85] and 3-4 GPa for PLA. The high modulus makes PLA unsuitable for membranes which should be exposed to mechanical strain. 


\section{Preparation of porous polymeric membranes for organs- on-chips}

The porous membranes in OOCs should not only provide support for the cells, but should also allow communication of the cells between the chip compartments and allow nutrients and other factors to reach all the cells. For example, in the lung-on-a-chip from Huh et al. [12], porous membranes were applied, so neutrophils could pass the membrane and reach the lung cells on the other side of the membrane to initiate an immune response against Escherichia coli. In general, membranes in OOCs are made porous by two methods that allow for good control of the pore size, shape and distribution (Table 3).

Table 3. Fabrication techniques used to prepare porous membranes for OOCs.

\begin{tabular}{ll} 
Fabrication technique & Polymer \\
\hline \multirow{2}{*}{ Soft lithography } & PDMS [1,6,7,10,11,13,18,19,38,42,47] \\
& PEG-DA [52] \\
& PLA [33] \\
\hline Track etching & PC [4,5,8,9,16,23,25,27,29,41,43,44,51] \\
& PET [2,15,21,23,26,28,39,48,53] \\
\hline Injection moulding in a microfluidic & PDMS [52] \\
\cline { 2 - 2 } chip with micropillars & PUA [52] \\
\hline Electrospinning & PCL [34] \\
\hline
\end{tabular}

\subsection{Soft lithography}

The most common method to fabricate components of the OOCs is soft lithography. It is used for both the 'rigid' chip itself $[1,2,5-7,9,11-$ $13,15,16,18,19,21-26,28,29,33,36,38,40,47]$ and the flexible porous membrane that supports the cells $[1,6,7,10,11,13,18,19,33,38,42,47]$. Soft lithography is very suitable to prepare porous membranes for OOCs (Table 3). Huh et al. [1] pushed the micropillars of a silicon master through an uncured PDMS film (Figure 2). The punctured film was cured to create a porous PDMS membrane. The size, distribution and amount of the 
pores were thus all controlled by the micropillars on the silicon master. This method results in a low pore tortuosity as the pores are always perpendicular to the membrane surface. This is a great advantage because the migration and transport of cells and nutrients are optimised. A similar approach was used by Pensabene et al. [33], who used a patterned mould with microneedles made of poly(vinyl alcohol) to prepare porous PLA membranes for PDMS-based OOCs containing endothelial cells.

A

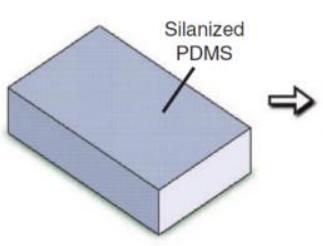

C

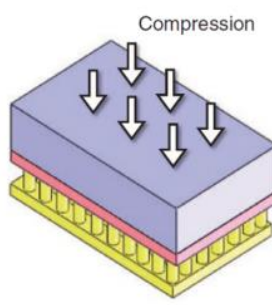

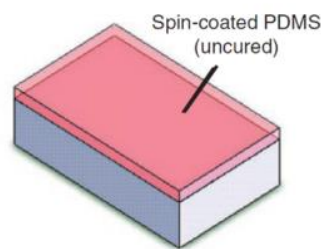

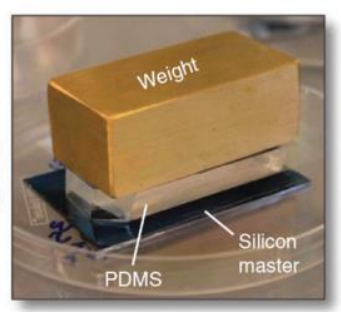

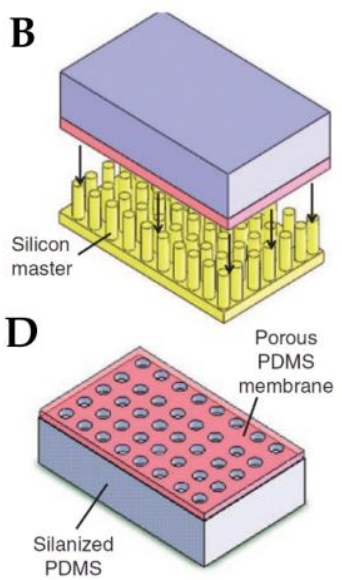

Figure 2. Preparation of porous PDMS membranes by soft lithography. (A) A slab of PDMS is cured and silanised. Uncured PDMS is spin-coated on top of the silanised side to form a $10 \mu \mathrm{m}$ thin film. (B) The PDMS is placed on top of a silicon master containing micropillars with the diameter of the pores. (C) The entire assembly is compressed, in order for the micropillars to completely penetrate the uncured PDMS. The PDMS is cured and (D) after removing the silicon master and silanised PDMS, a porous membrane remains. Adapted from Huh et al. [1]. 
Flat and microstructured polymeric membranes in organs-on-chips

\subsection{Track etching}

Many of the porous membranes from OOCs originate from commercial inserts or filter membranes made of PC and PET which were prepared by track etching $[2,4,5,21,23,25-27,29,39,41,43,44,51]$. Track etching involves applying either electrons, heavy ions, X-ray irradiation or UV light, which pass at predefined spaces of a mask. For example, ion track etching (Figure 3) uses heavy ions to cleave polymer chains at specific spots to form cylindrical tracks of degraded polymer, perpendicular to the surface of the film. After irradiation, the tracks have to be opened. A conventional method is wet-chemical etching which uses chemicals or etchants, often acids or bases such as sodium hydroxide, which may not be preferable for some polymers [78,89-91]. By changing the etchant concentration, etching temperature and etching duration, pore sizes can be tailored from nanometres to micrometres (Figure 3D). With the polymer removed, cylindrical pores remain with low tortuosity. The control over pore size, shape and density makes track etching a very relevant method to produce porous polymer membranes which are very suitable for transport or migration studies.

A

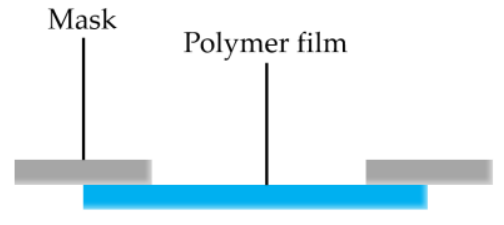

C

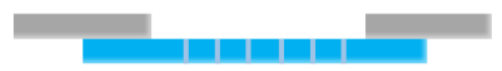

B

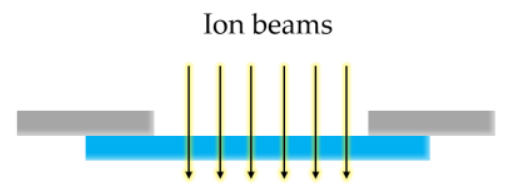

D

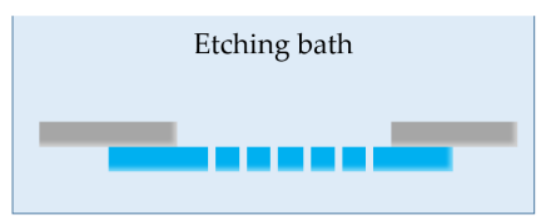

Figure 3. Ion track etching. (A) A polymer film is covered by a mask. (B) Ion beams pass through the opening(s) in the mask and locally penetrate the polymer film. (C) Polymer chains have been locally degraded by the ion beams. (D) By placing the film in a bath with etching agents, the degraded polymer is removed, and pores are formed. 


\section{Microfabrication of membranes for use in future organs- on-chips}

As we discussed earlier, to create reliable and representative OOCs, we need to apply membranes with surface topography closely mimicking the topography of the tissue/organ to be investigated. In this section, we will discuss the state of the art in developing porous, microstructured membranes which could replace the flat, non-microstructured membranes currently used in OOCs. Realistic cell behaviour could be stimulated by culturing cells on porous membranes that also include microstructures which resemble the configuration of the cells in the body. To prepare such porous, microstructured membranes, the mentioned methods to produce porous membranes can be combined with microfabrication. Microfabrication consists of a set of techniques to prepare micrometre-scale structures. Microfabrication is already applied in OOC technology to prepare the chips $[1,18,19]$, but not used to fabricate porous, microstructured membranes. Taking advantage of microfabrication as it is used in other fields of biomedical engineering could accomplish the three-dimensional organisation of cells. Microfabrication could provide a robust, reproducible microenvironment that is independent of the culturing conditions of the cells. Microfabrication would make it possible to prepare structures that mimic different versions of a particular tissue, such as large or small blood vessels, duodenum and small intestine, or specific pathologies where the shape of the tissue is abnormal.

\subsection{Soft lithography replica moulding}

As mentioned before, soft lithography is used for fabrication of flat porous membranes, as well as the chips of OOCs. However, it can also be used for producing microstructured membranes. The most common soft lithography technique for OOCs is replica moulding (Figure 4A) $[1,15,18,19,33]$. In the case of OOCs, uncured PDMS is deposited on a microstructured mould and cured (Figure 4A.I-III). After removal from 
Flat and microstructured polymeric membranes in organs-on-chips

the mould, the PDMS contains negative copies of the microstructures of the mould (Figure 4A.IV).

A

I.

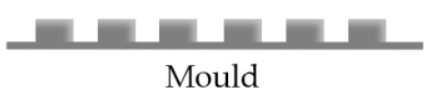

II.

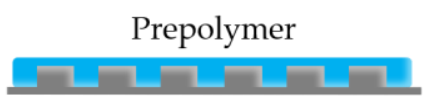

III.

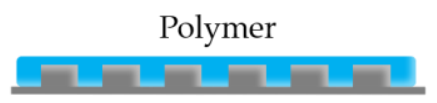

IV.

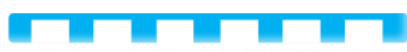

B

I.

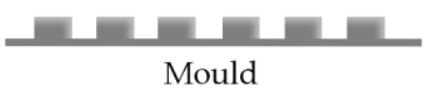

II.

Polymer solution

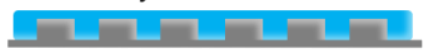

III.

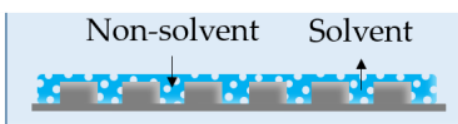

IV.

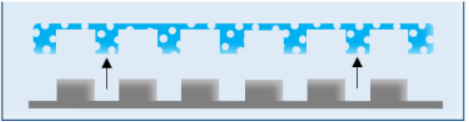

Figure 4. (A) Schematic representation of soft lithography replica moulding. (I) A microstructured mould. (II) The mould is filled with prepolymer. (III) The prepolymer is then cured. Note: depending on the polymer, curing may not be necessary. (IV) Removing the mould reveals a microstructured polymer film. (B) The principle of phase separation micromoulding. (I) A microstructured mould. (II) The mould is filled with a polymer solution consisting of polymer dissolved in a solvent. (III) The mould and polymer solution are submerged in a bath of non-solvent. Liquid-induced phase separation occurs through the exchange of non-solvent and solvent. This exchange solidifies the polymer solution and creates pores. (IV) The porous, microstructured membrane releases from the mould.

Although simple and accurate, there are limitations to the capabilities of soft lithography. Microstructures will only appear on one side of the membrane, while the other side remains flat (Figure 4A.IV). Pores could be included, but their position and orientation would be limited. Using micropillars as discussed before, pores can be created perpendicular to the surface of a flat membrane. For the purpose of a porous membrane with microwells, the walls of the microwells would not contain pores as the mould cannot be made with micropillars in the horizontal direction. 
Flat and microstructured polymeric membranes in organs-on-chips

Another approach is to combine soft lithography with other pore-forming methods such as particulate leaching. Vozzi et al. [92] prepared scaffolds of PLGA by different soft lithography techniques. By dispersing glucose grains in the polymer solution which were leached afterwards, it was possible to introduce pores into the scaffolds. Likewise, soft lithography could be used to prepare porous, microstructured membranes.

\subsection{Phase separation micromoulding}

Phase separation micromoulding (PS $\mu \mathrm{M})$ (Figure 4B) enables the introduction of porosity and patterns into membranes in one step by inducing phase separation, most often liquid-induced phase separation $[72,73,93,94]$, of a polymer solution on a patterned mould. The appropriate polymer solution is cast onto a micro- and/or nanostructured master mould (prepared by technologies derived from microelectronics and photolithography). During liquid-induced phase separation, solvent and non-solvent liquid exchange initiate phase separation until the polymer solution contains sufficient non-solvent to precipitate. As a result, the porous membrane solidifies and releases from the mould. By selecting the right solvent/non-solvent system with the specific polymer, the porosity of the resulting membrane can be tuned. The shape, size and distribution of the microstructures that are imprinted in the membranes can be altered by changing the design of the mould to meet the requirements of a particular application $[9,72,73,93]$.

\subsection{Thermoforming}

Porous, microstructured membranes can be made by thermoforming [78,89-91,95]. With most thermoforming methods, a polymer film is placed on a plate which acts as the mould and contains the microstructures that have to be replicated [78,89-91,95]. It is then heated to a soft but still solid state, after which a force is applied to shape the film to the mould. This force can be applied in several ways, most commonly by air pressure (Figure 5A), a vacuum (Figure 5B) or a mechanical force (Figure 5C,D). The properties of the film may affect the outcome. For 
- Chapter 2 -

Flat and microstructured polymeric membranes in organs-on-chips

example, densely cured polymer networks may not deform permanently by thermoforming.

A Pressure thermoforming
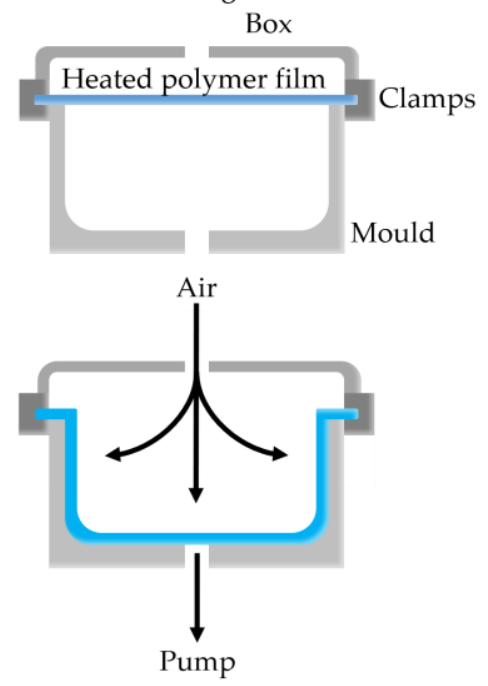

CMechanical thermoforming
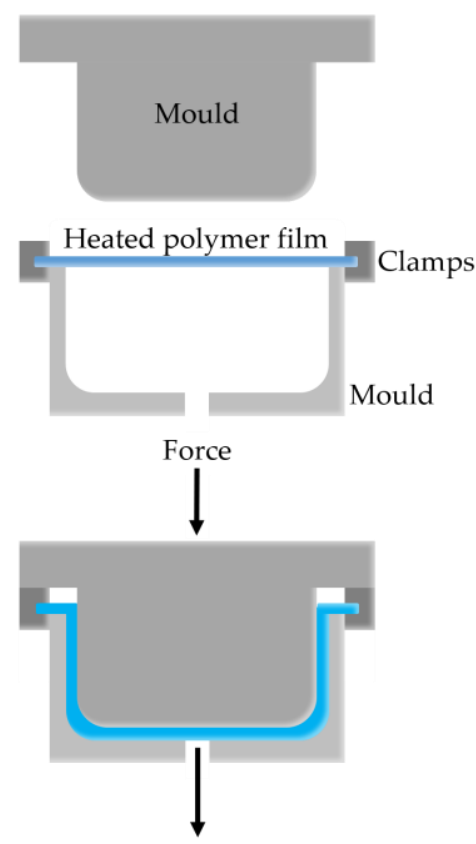

B Vacuum thermoforming
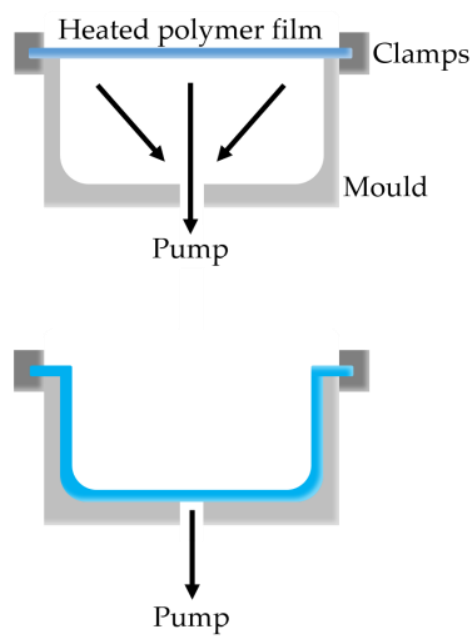

D Micro back moulding

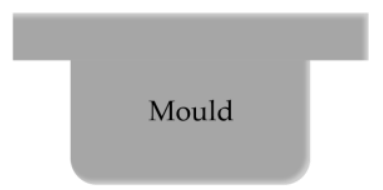

Heated polymer film

Thermoplastic polymer

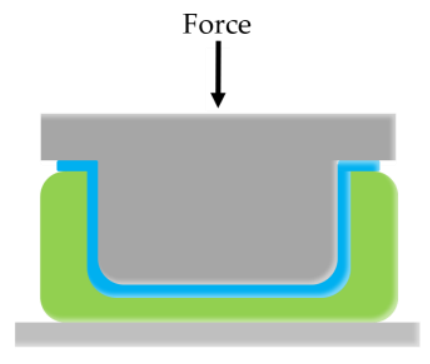


Flat and microstructured polymeric membranes in organs-on-chips

Figure 5. Schematic of thermoforming techniques. (A) Pressure thermoforming: a compressed gas can be released which exerts a pressure that pushes a preheated plastic sheet, such as a polymer film or membrane, against the mould. (B) Vacuum thermoforming: a vacuum can be applied on the other side of a preheated plastic sheet. The lower pressure forces the sheet towards the mould. (C) Mechanical thermoforming: a preheated plastic sheet is placed between complementary moulds, i.e. a positive and negative version of the mould, on both sides of the sheet. These are pressed against each other, shaping the plastic sheet to the moulds. (D) Micro back moulding: a preheated plastic sheet is placed on a thick layer of elastomeric or thermoplastic polymer. Upon compression by a mould, both the plastic sheet and the polymer layer beneath it are shaped to the mould.

To create porous, microstructured membranes, thermoforming is often used in conjunction with ion track etching [78,89-91]. Usually, polymer films are first track-etched locally and are then thermoformed into a threedimensional shape. Finally, the pores are opened by wet-chemical etching. Particular attention is needed because the films are stretched during thermoforming. By opening the pores afterwards, the effect of stretch on pore size and shape is minimal [78,89-91].

Thermoforming has been used to prepare an alternative Boyden chamber for studies on trans-endothelial transport [78]. By combining ion track etching with thermoforming, porous PC membranes with a semicircular microchannel were prepared which closely resembled the threedimensional shape of a blood vessel. Indeed, these kinds of systems allow for a more realistic study of, for example, trans-endothelial transport of drugs [78].

Hou et al. [96] compression-moulded a mixture of dispersed salt particles in PCL. Compression moulding is very similar to thermoforming. However, a clear distinction has to be made. Often temperatures are much higher during compression moulding than thermoforming, heating a polymer above the melting point to a liquid state. When the polymer is in the liquid state, it is compressed into a film between two plates and afterwards cooled. By using microstructured moulds instead of flat plates 
Flat and microstructured polymeric membranes in organs-on-chips

during compression moulding, the films can be made to contain microstructures, and pores can be formed by particulate leaching. Buitinga et al. [95] prepared flat films of poly(ethylene oxide terephthalate)-poly(butylene terephthalate) (PEOT/PBT) block copolymer by solvent casting. This was followed by evaporation of the solvent to form a dense film. The films were then thermoformed to obtain a microwell array where human islets of Langerhans were cultured separately without adhesion to the film or each other. They also prepared porous PEOT/PBT membranes via electrospinning which were afterwards thermoformed.

\subsection{Electrospinning}

Electrospinning can also yield porous, microstructured membranes in one step. Electrospinning involves charging a polymer solution or polymer melt by a high voltage (Figure 6A). The charge attracts the polymer to a ground plate, where it is collected. Because changes in the charge occur between the needle and the collection substrate, the polymer flow will continuously change direction and thus spread over the ground plate, resulting in a fibre mesh. The amount of space between fibres can be changed by controlling the density and amount of the polymer solution that is deposited, effectively controlling the porosity, pore size and thickness of the membrane $[98,99]$. The same principle can be applied to fabricate porous, microstructured membranes by electrospinning over microstructured moulds (Figure 6B). Cheng et al. [100] spun PLA fibres on PDMS moulds containing different pyramid-like microstructures. Distinct PLA structures were obtained by changing the dimensions of and spacing between the pyramids. 
- Chapter 2 -

Flat and microstructured polymeric membranes in organs-on-chips

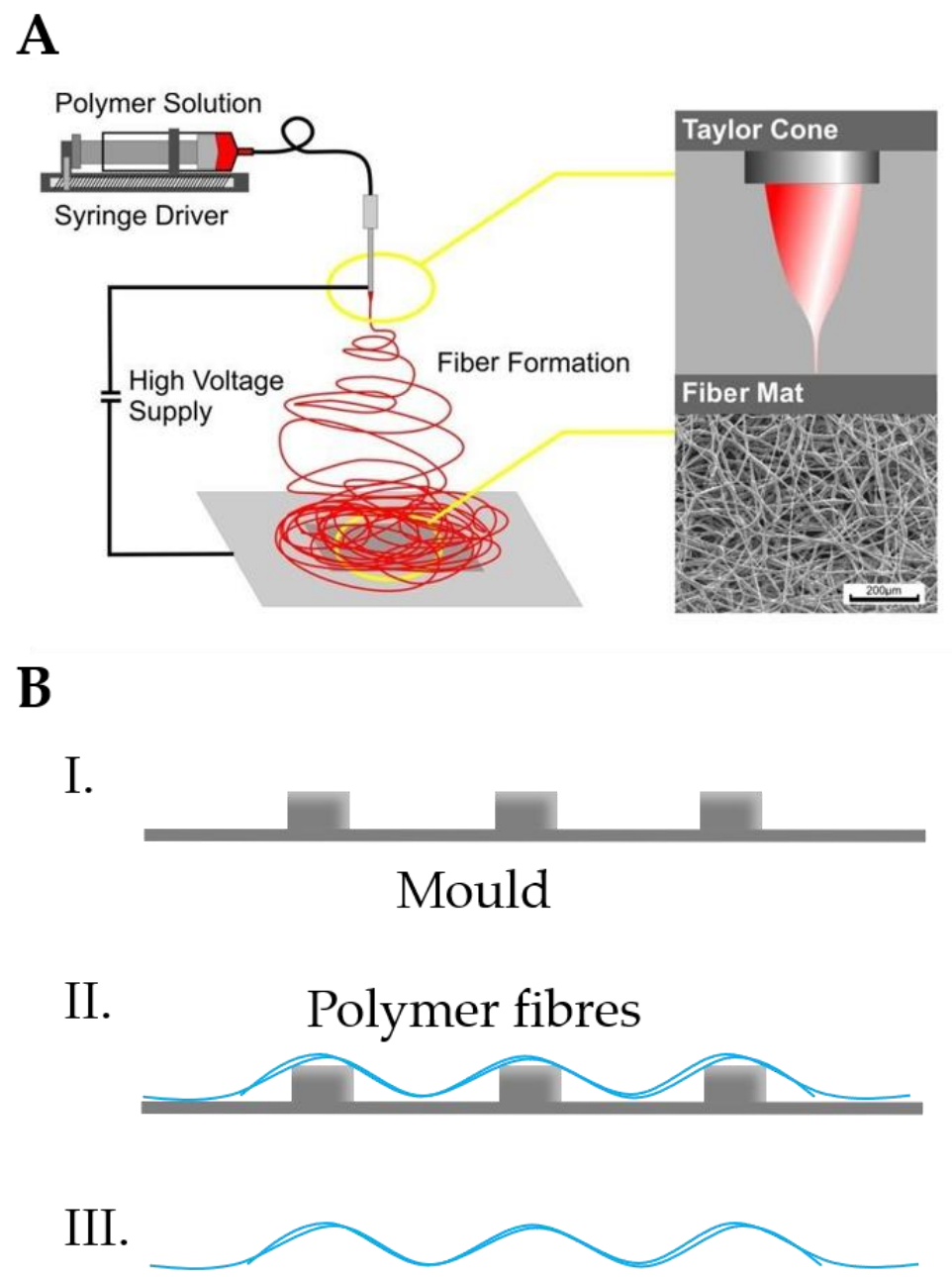

Figure 6. Schematic representation of electrospinning (A) a porous, flat and (B) a porous, microstructured membrane. (A) A polymer solution is charged with a high voltage. Thin fibres are spun from the polymer solution, which are attracted to a substrate where they are collected. Adapted from Wallace et al. [97]. (B) (I) A microstructured silicon wafer mould. (II) Fibres are electrospun on top of the mould. (III) The mould is removed, and a microstructured mesh of polymer fibres remains. 
Flat and microstructured polymeric membranes in organs-on-chips

\section{Conclusion}

There are significant issues concerning the development of new drugs. In vitro and in vivo models often do not predict drug responses in humans and clinical trials do not account for individual differences in response to drugs. Recently, OOCs have been developed to overcome these issues. OOCs are small microfluidic chips that contain cells and feature characteristics of an organ, such as fluid flow and mechanical forces, in order to closely mimic organ function. Indeed, OOCs have proved that cells show more realistic behaviour in an environment which resembles their native tissue than in traditional static culturing systems. In most OOCs, cells are cultured on a porous polymer membrane. Although OOCs confirm that mimicking the cell environment is beneficial, there is little attention paid to the membranes used in OOCs. Membrane properties such as stiffness and surface roughness can greatly influence cells, such as their adherence and growth. Moreover, cells react to the shape of the membrane, such as the presence of microstructures.

Most membranes in OOCs are made from only a few polymers, which often require treatments or coatings to allow cells to adhere and grow. Moreover, most of the polymers cannot be stretched, and thus many OOCs cannot provide mechanical strain to the cells, despite the often dynamic nature of the native tissues the OOCs try to mimic.

The pores in the membranes are fabricated with two methods in particular, i.e. soft lithography using a micropillar mould or ion track etching of a polymer film. Both offer good control over pore size and density, and result in low pore tortuosity. In fact, commercial membranes made by ion track etching are used in many OOCs.

There is compelling evidence that surface microstructures have a significant effect on cells and can stimulate realistic cell behaviour. Despite this, membranes in current OOCs are flat, while there is great potential for microfabrication of three-dimensional environments. Microfabrication provides simple processing techniques for the preparation of microstructured membranes from a wide variety of materials. 
Flat and microstructured polymeric membranes in organs-on-chips

Soft lithography and thermoforming have already been used to prepare porous, microstructured membranes for purposes outside the field of OOCs. In these cases, thermoforming is combined with ion track etching to create the microstructures and pores, respectively. Soft lithography can be used to produce microstructures and has to be complemented with techniques such as particulate leaching to prepare porous, microstructured membranes. PS $\mu \mathrm{M}$ is very similar to soft lithography replica moulding. However, PS $\mathrm{MM}$ can create both pores and microstructures in a single step. Porous membranes with microstructures can also be produced by electrospinning in conjunction with thermoforming or electrospinning alone.

Thus far, evidence concerning the effects of membrane characteristics, polymer properties and surface microstructures on cells has been neglected in the field of OOCs. However, the desire to improve OOCs demands that all elements of the system are optimised, including the membranes. To do so, we need to understand more about the underlying mechanisms of cell-substrate interactions and how they affect cell behaviour. Moreover, although it is becoming clear that each cell type has preferences, it is not known what those ideal substrate properties are. By mimicking the stiffness of the native ECM, while providing a collagen coating to stimulate cell adhesion, it is already possible to direct MSC differentiation [64], indicating that properly mimicking the ECM is beneficial. Conversely, not having the correct properties of a substrate might give interfering cues to a cell. While most studies make use of only a few polymers, often with different properties from the tissue of interest, other studies show less common polymers with superior cytocompatibility and/or mechanical properties, which should be explored. Surface roughness, porosity, cell adhesive properties and the inclusion of microstructures spanning multiple cells should also be optimised. However, these studies could very easily become impractical with many variables to consider.

Therefore, systematic studies with high-throughput screening are needed, such as those using chips with numerous geometries to analyse cell responses to surface topography $[65,66]$. This could also provide 
Flat and microstructured polymeric membranes in organs-on-chips

information about the preferred spacing of topographies which could be used when considering the surface roughness, pore size and porosity. Systems such as that of Lee et al. [69], where different ECM proteins can be attached to the substrate surface, could provide tools to better investigate cell responses to substrate stiffness for example, with limited influence of the substrate chemistry. Combining these kinds of methods could provide high-throughput chips which can systematically screen for the best topography, stiffness, surface roughness and other properties to come closer to the native ECM. Lastly, incorporating microstructures with similar shapes and dimensions as the native tissue would further mimic the spatial organisation of cells in the body.

Microfabrication can be applied to create a wide range of porous, microstructured membranes acting as controllable, physiologically accurate representations of native tissue morphology. Depending on the requirements for the membrane or limitations of the polymer, microfabrication offers different techniques for creating both the pores and microstructures to suit these needs. The relative simplicity of microfabrication techniques makes them viable tools for the preparation of a new generation of membranes to be used for further improvement of OOCs.

\section{References}

1. Huh, D.; Kim, H.J.; Fraser, J.P.; Shea, D.E.; Khan, M.; Bahinski, A.; Hamilton, G.A.; Ingber, D.E. Microfabrication of human organs-on-chips. Nat. Protoc. 2013, 8(11), 2135-2157. DOI: 10.1038/nprot.2013.137.

2. Abhyankar, V.V.; Wu, M.; Koh, C.Y.; Hatch, A.V. A Reversibly Sealed, Easy Access, Modular (SEAM) Microfluidic Architecture to Establish In Vitro Tissue Interfaces. PloS ONE. 2016, 11(5), e0156341. DOI: 10.1371/journal.pone.0156341.

3. Bavli, D.; Prill, S.; Ezra, E.; Levy, G.; Cohen, M.; Vinken, M.; Vanfleteren, J.; Jaeger, M.; Nahmias, Y. Real-time monitoring of metabolic function in liver-onchip microdevices tracks the dynamics of mitochondrial dysfunction. Proc. Natl Acad. Sci. USA. 2016, 113(16), E2231-E2240. DOI: 10.1073/pnas.1522556113.

4. Brown, J.A.; Codreanu, S.G.; Shi, M.; Sherrod, S.D.; Markov, D.A.; Neely, M.D.; Britt, C.M.; Hoilett, O.S.; Reiserer, R.S.; Samson, P.C.; McCawley, L.J.; Webb, D.J.; Bowman, A.B.; McLean, J.A.; Wikswo, J.P. Metabolic consequences of inflammatory disruption of the blood-brain barrier in an organ-on-chip model of 


\section{- Chapter 2 -}

Flat and microstructured polymeric membranes in organs-on-chips

the human neurovascular unit. J. Neuroinflamm. 2016, 13(1), 306. DOI: 10.1186/s12974-016-0760-y.

5. Chen, C.; Mehl, B.T.; Sell, S.A.; Martin, R.S. Use of electrospinning and dynamic air focusing to create three-dimensional cell culture scaffolds in microfluidic devices. Analyst. 2016, 141(18), 5311-5320. DOI: 10.1039/c6an01282e.

6. Chen, Y.; Chan, H.N.; Michael, S.A.; Shen, Y.; Chen, Y.; Tian, Q.; Huang, L.; Wu, $\mathrm{H}$. A microfluidic circulatory system integrated with capillary-assisted pressure sensors. Lab Chip. 2017, 17(4), 653-662. DOI: 10.1039/c6lc01427e.

7. Di Caprio, G.; Stokes, C.; Higgins, J.M.; Schonbrun, E. Single-cell measurement of red blood cell oxygen affinity. Proc. Natl Acad. Sci. USA. 2015, 112(32), 99849989. DOI: 10.1073/pnas.1509252112.

8. Eain, M.M.G.; Baginska, J.; Greenhalgh, K.; Fritz, J.V.; Zenhausern, F.; Wilmes, P. Engineering solutions for representative models of the gastrointestinal humanmicrobe interface. Engineering. 2017, 3, 60-65. DOI: 10.1016/J.ENG.2017.01.011.

9. Gao, D.; Liu, H.; Lin, J.M.; Wang, Y.; Jiang, Y. Characterization of drug permeability in Caco-2 monolayers by mass spectrometry on a membrane-based microfluidic device. Lab Chip. 2013, 13(5), 978-985. DOI: 10.1039/c2lc41215b.

10. Huh, D.; Hamilton, G.A.; Ingber, D.E. From 3D cell culture to organs-onchips. Trends Cell Biol. 2011, 21(12), 745-754. DOI: 10.1016/j.tcb.2011.09.005.

11. Huh, D.; Leslie, D.C.; Matthews, B.D.; Fraser, J.P.; Jurek, S.; Hamilton, G.A.; Thorneloe, K.S.; McAlexander, M.A.; Ingber, D.E. A human disease model of drug toxicity-induced pulmonary edema in a lung-on-a-chip microdevice. Sci. Trans. Med. 2012, 4(159), 159ra147. DOI: 10.1126/scitranslmed.3004249.

12. Huh, D.; Matthews, B.D.; Mammoto, A.; Montoya-Zavala, M.; Hsin, H.Y.; Ingber, D.E. Reconstituting organ-level lung functions on a chip. Science. 2010, 328(5986), 1662-1668. DOI: 10.1126/science.1188302.

13. Huh, D.; Torisawa, Y.S.; Hamilton, G.A.; Kim, H.J.; Ingber, D.E. Microengineered physiological biomimicry: organs-on-chips. Lab Chip. 2012, 12(12), 2156-2164. DOI: 10.1039/c2lc40089h.

14. Illa, X.; Vila, S.; Yeste, J.; Peralta, C.; Gracia-Sancho, J.; Villa, R. A novel modular bioreactor to in vitro study the hepatic sinusoid. PloS ONE. 2014, 9(11), e111864. DOI: 10.1371/journal.pone.0111864.

15. Jang, K.J.; Mehr, A.P.; Hamilton, G.A.; McPartlin, L.A.; Chung, S.; Suh, K.Y.; Ingber, D.E. Human kidney proximal tubule-on-a-chip for drug transport and nephrotoxicity assessment. Integr. Biol. 2013, 5(9), 1119-1129. DOI: 10.1039/c3ib40049b.

16. Jie, M.; Li, H-F.; Lin, L.; Zhang, J.; Lin, J-M. Integrated microfluidic system for cell co-culture and simulation of drug metabolism. RSC Adv. 2016, 6, 54 564-54 572. DOI: 10.1039/C6RA10407J.

17. Kang, Y.B.; Sodunke, T.R.; Lamontagne, J.; Cirillo, J.; Rajiv, C.; Bouchard, M.J.; Noh, M. Liver sinusoid on a chip: Long-term layered co-culture of primary rat hepatocytes and endothelial cells in microfluidic platforms. Biotechnol. Bioeng. 2015, 112(12), 2571-2582. DOI: 10.1002/bit.25659. 


\section{- Chapter 2 -}

Flat and microstructured polymeric membranes in organs-on-chips

18. Kim, H.J.; Huh, D.; Hamilton, G.; Ingber, D.E. Human gut-on-a-chip inhabited by microbial flora that experiences intestinal peristalsis-like motions and flow. Lab Chip. 2012, 12(12), 2165-2174. DOI: 10.1039/c2lc40074j.

19. Kim, H.J.; Ingber, D.E. Gut-on-a-Chip microenvironment induces human intestinal cells to undergo villus differentiation. Integr. Biol. 2013, 5(9), 1130 1140. DOI: 10.1039/c3ib40126j.

20. Lee, H.; Cho, D.W. One-step fabrication of an organ-on-a-chip with spatial heterogeneity using a 3D bioprinting technology. Lab Chip. 2016, 16(14), 26182625. DOI: 10.1039/c6lc00450d.

21. Lee, H.; Kim, D.S.; Ha, S.K.; Choi, I.; Lee, J.M.; Sung, J.H. A pumpless multiorgan-on-a-chip (MOC) combined with a pharmacokinetic-pharmacodynamic (PK-PD) model. Biotechnol. Bioeng. 2017, 114(2), 432-443. DOI: 10.1002/bit.26087.

22. Lee, J.S.; Romero, R.; Han, Y.M.; Kim, H.C.; Kim, C.J.; Hong, J.S.; Huh, D. Placenta-on-a-chip: a novel platform to study the biology of the human placenta. J Matern Fetal Neonatal Med. 2016, 29(7), 1046-1054. DOI: 10.3109/14767058.2015.1038518.

23. Lee, S.; Jin, S.P.; Kim, Y.K.; Sung, G.Y.; Chung, J.H.; Sung, J.H. Construction of 3D multicellular microfluidic chip for an in vitro skin model. Biomed. Microdevices. 2017, 19(2), 22. DOI: 10.1007/s10544-017-0156-5.

24. Li, X.; Brooks, J.C.; Hu, J.; Ford, K.I.; Easley, C.J. 3D-templated, fully automated microfluidic input/output multiplexer for endocrine tissue culture and secretion sampling. Lab Chip. 2017, 17(2), 341-349. DOI: 10.1039/c6lc01201a.

25. Li, Z.; Guo, Y.; Yu, Y.; Xu, C.; Xu, H.; Qin, J. Assessment of metabolismdependent drug efficacy and toxicity on a multilayer organs-on-a-chip. Integr. Biol. 2016, 8(10), 1022-1029. DOI: 10.1039/c6ib00162a.

26. Loskill, P.; Sezhian, T.; Tharp, K.M.; Lee-Montiel, F.T.; Jeeawoody, S.; Reese, W.M.; Zushin, P.H.; Stahl, A.; Healy, K.E. WAT-on-a-chip: a physiologically relevant microfluidic system incorporating white adipose tissue. Lab Chip. 2017, 17(9), 1645-1654. DOI: 10.1039/c6lc01590e.

27. Ma, Y.; Pan, J.Z.; Zhao, S.P.; Lou, Q.; Zhu, Y.; Fang, Q. Microdroplet chain array for cell migration assays. Lab Chip. 2016, 16(24), 4658-4665. DOI: 10.1039/c6lc00823b.

28. Maoz, B.M.; Herland, A.; Henry, O.; Leineweber, W.D.; Yadid, M.; Doyle, J.; Mannix, R.; Kujala, V.J.; FitzGerald, E.A.; Parker, K.K.; Ingber, D.E. Organs-onChips with combined multi-electrode array and transepithelial electrical resistance measurement capabilities. Lab Chip. 2017, 17(13), 2294-2302. DOI: 10.1039/c7lc00412e.

29. Maschmeyer, I.; Hasenberg, T.; Jaenicke, A.; Lindner, M.; Lorenz, A.K.; Zech, J.; Garbe, L.A.; Sonntag, F.; Hayden, P.; Ayehunie, S.; Lauster, R.; Marx, U.; Materne, E.M. Chip-based human liver-intestine and liver-skin co-cultures--A first step toward systemic repeated dose substance testing in vitro. Eur. J. Pharm. Biopharm. 2015, 77-87. DOI: 10.1016/j.ejpb.2015.03.002. 


\section{- Chapter 2 -}

Flat and microstructured polymeric membranes in organs-on-chips

30. McCain, M.L.; Sheehy, S.P.; Grosberg, A.; Goss, J.A.; Parker, K.K. Recapitulating maladaptive, multiscale remodeling of failing myocardium on a chip. Proc. Natl Acad. Sci. USA. 2013, 110(24), 9770-9775. DOI: 10.1073/pnas.1304913110.

31. Mermoud, Y.; Felder, M.; Stucki, J.D.; Stucki, A.O.; Guenat, O.T. Microimpedance tomography system to monitor cell activity and membrane movements in a breathing lung-on-chip. Sens. Actuators, B. 2018, 255, 3647-3653. DOI: 10.1016/j.snb. 2017.09.192.

32. Park, J.; Lee, B.K.; Jeong, G.S.; Hyun, J.K.; Lee, C.J.; Lee, S.H. Three-dimensional brain-on-a-chip with an interstitial level of flow and its application as an in vitro model of Alzheimer's disease. Lab Chip. 2015, 15(1), 141-150. DOI: 10.1039/c4lc00962b.

33. Pensabene, V.; Costa, L.; Terekhov, A.Y.; Gnecco, J.S.; Wikswo, J.P.; Hofmeister, W.H. Ultrathin Polymer Membranes with Patterned, Micrometric Pores for Organs-on-Chips. ACS Appl. Mater. Interfaces. 2016, 8(34), 22629-22636. DOI: 10.1021/acsami.6b05754.

34. Pensabene, V.; Crowder, S.W.; Balikov, D.A.; Lee, J.B.; Sung, H.J. Optimization of electrospun fibrous membranes for in vitro modeling of blood-brain barrier. Conf. Proc. IEEE Eng. Med. Biol. Soc. 2016, 125-128. DOI: 10.1109/EMBC.2016.7590656.

35. Phan, D.; Wang, X.; Craver, B.M.; Sobrino, A.; Zhao, D.; Chen, J.C.; Lee, L.; George, S.C.; Lee, A.P.; Hughes, C. A vascularized and perfused organ-on-a-chip platform for large-scale drug screening applications. Lab Chip. 2017, 17(3), 511520. DOI: 10.1039/c6lc01422d.

36. Punde, T.H.; Wu, W.H.; Lien, P.C.; Chang, Y.L.; Kuo, P.H.; Chang, M.D.; Lee, K.Y.; Huang, C.D.; Kuo, H.P.; Chan, Y.F.; Shih, P.C.; Liu, C.H. A biologically inspired lung-on-a-chip device for the study of protein-induced lung inflammation. Integr. Biol. 2015, 7(2), 162-169. DOI: 10.1039/c4ib00239c.

37. Qian, F.; Huang, C.; Lin, Y.D.; Ivanovskaya, A.N.; O'Hara, T.J.; Booth, R.H.; Creek, C.J.; Enright, H.A.; Soscia, D.A.; Belle, A.M.; Liao, R.; Lightstone, F.C.; Kulp, K.S.; Wheeler, E.K. Simultaneous electrical recording of cardiac electrophysiology and contraction on chip. Lab Chip. 2017, 17(10), 1732-1739. DOI: $10.1039 /$ c7lc00210f.

38. Riahi, R.; Shaegh, S.A.; Ghaderi, M.; Zhang, Y.S.; Shin, S.R.; Aleman, J.; Massa, S.; Kim, D.; Dokmeci, M.R.; Khademhosseini, A. Automated microfluidic platform of bead-based electrochemical immunosensor integrated with bioreactor for continual monitoring of cell secreted biomarkers. Sci. Rep. 2016, 6, 24598. DOI: 10.1038/srep24598.

39. Shim, K.Y.; Lee, D.; Han, J.; Nguyen, N.T.; Park, S.; Sung, J.H. Microfluidic guton-a-chip with three-dimensional villi structure. Biomedical Microdevices. 2017, 19(2), 37. DOI: 10.1007/s10544-017-0179-y.

40. Shin, S.R.; Zhang, Y.S.; Kim, D.J.; Manbohi, A.; Avci, H.; Silvestri, A.; Aleman, J.; Hu, N.; Kilic, T.; Keung, W.; Righi, M.; Assawes, P.; Alhadrami, H.A.; Li, R.A.; Dokmeci, M.R.; Khademhosseini, A. Aptamer-Based Microfluidic Electrochemical Biosensor for Monitoring Cell-Secreted Trace Cardiac 
Flat and microstructured polymeric membranes in organs-on-chips

Biomarkers. Anal. Chem. 2016, 88(20), 10019-10027. DOI: 10.1021/acs.analchem.6b02028.

41. Sticker, D.; Rothbauer, M.; Lechner, S.; Hehenberger, M.T.; Ertl, P. Multi-layered, membrane-integrated microfluidics based on replica molding of a thiol-ene epoxy thermoset for organ-on-a-chip applications. Lab on a chip. 2015, 15(24), 4542-4554. DOI: 10.1039/c5lc01028d.

42. Stucki, A.O.; Stucki, J.D.; Hall, S.R.; Felder, M.; Mermoud, Y.; Schmid, R.A.; Geiser, T.; Guenat, O.T. A lung-on-a-chip array with an integrated bio-inspired respiration mechanism. Lab Chip. 2015, 15(5), 1302-1310. DOI: 10.1039/c4lc01252f.

43. van der Helm, M.W.; Odijk, M.; Frimat, J.P.; van der Meer, A.D.; Eijkel, J.; van den Berg, A.; Segerink, L.I. Direct quantification of transendothelial electrical resistance in organs-on-chips. Biosens. Bioelectron. 2016, 85, 924-929. DOI: 10.1016/j.bios.2016.06.014.

44. Wang, Y.I.; Abaci, H.E.; Shuler, M.L. Microfluidic blood-brain barrier model provides in vivo-like barrier properties for drug permeability screening. Biotechnol. Bioeng. 2017, 114(1), 184-194. DOI: 10.1002/bit.26045.

45. Weibel, D.B.; Diluzio, W.R.; Whitesides, G.M. Microfabrication meets microbiology. Nat. Rev. Microbiol. 2007, 5(3), 209-218. DOI: 10.1038/nrmicro1616.

46. Yu, F.; Deng, R.; Hao Tong, W.; Huan, L.; Chan Way, N.; IslamBadhan, A.; Iliescu, C.; $\mathrm{Yu}, \mathrm{H}$. A perfusion incubator liver chip for 3D cell culture with application on chronic hepatotoxicity testing. Sci. Rep. 2017, 7(1), 14528. DOI: 10.1038/s41598017-13848-5.

47. Zhang, Y.S.; Aleman, J.; Shin, S.R.; Kilic, T.; Kim, D.; Mousavi Shaegh, S.A.; Massa, S.; Riahi, R.; Chae, S.; Hu, N.; Avci, H.; Zhang, W.; Silvestri, A.; Sanati Nezhad, A.; Manbohi, A.; De Ferrari, F.; Polini, A.; Calzone, G.; Shaikh, N.; Alerasool, P.; ... Khademhosseini, A. Multisensor-integrated organs-on-chips platform for automated and continual in situ monitoring of organoid behaviors. Proc. Natl Acad. Sci. USA. 2017, 114(12), E2293-E2302. DOI: 10.1073/pnas.1612906114.

48. Zhu, L.; Xia, H.; Wang, Z.; Fong, E.L.; Fan, J.; Tong, W.H.; Seah, Y.P.; Zhang, W.; $\mathrm{Li}, \mathrm{Q}$; $\mathrm{Yu}, \mathrm{H}$. A vertical-flow bioreactor array compacts hepatocytes for enhanced polarity and functions. Lab Chip. 2016, 16(20), 3898-3908. DOI: 10.1039/c6lc00811a.

49. Mulder M. 1996 Basic principles of membrane technology. Dordrecht, The Netherlands: Springer Netherlands.

50. Marzorati, M.; Vanhoecke, B.; De Ryck, T.; Sadaghian Sadabad, M.; Pinheiro, I.; Possemiers, S.; Van den Abbeele, P.; Derycke, L.; Bracke, M.; Pieters, J.; Hennebel, T.; Harmsen, H.J.; Verstraete, W.; \& Van de Wiele, T. The HMI ${ }^{\mathrm{TM}}$ module: a new tool to study the Host-Microbiota Interaction in the human gastrointestinal tract in vitro. BMC microbiology. 2014, 14, 133. DOI: 10.1186/1471-2180-14-133.

51. Pocock, K.J.; Gao, X.F.; Wang, C.X.; Priest, C.; Prestidge, C.A.; Mawatari, K.; Kitamori, T.; Thierry, B. Low-temperature bonding process for the fabrication of 


\section{- Chapter 2 -}

Flat and microstructured polymeric membranes in organs-on-chips

hybrid glass-membrane organ-on-a-chip devices. J. Micro-Nanolith Mem. 2016, 15, 044502. DOI: 10.1117/1. JMM.15.4.044502.

52. Tahk, D.; Paik, S.M.; Lim, J.; Bang, S.; Oh, S.; Ryu, H.; Jeon, N.L. Rapid large area fabrication of multiscale through-hole membranes. Lab Chip. 2017, 17(10), 18171825. DOI: 10.1039/c7lc00363c.

53. Casillo, S.M.; Peredo, A.P.; Perry, S.J.; Chung, H.H.; Gaborski, T.R. Membrane Pore Spacing Can Modulate Endothelial Cell-Substrate and Cell-Cell Interactions. ACS Biomater. Sci. Eng. 2017, 3(3), 243-248. DOI: 10.1021/acsbiomaterials.7b00055.

54. Wilson, C.J.; Clegg, R.E.; Leavesley, D.I.; Pearcy, M.J. Mediation of biomaterialcell interactions by adsorbed proteins: a review. Tissue Eng. 2005, 11(1-2), 1-18. DOI: 10.1089/ten.2005.11.1.

55. Ye, K.; Wang, X.; Cao, L.; Li, S.; Li, Z.; Yu, L.; Ding, J. Matrix Stiffness and Nanoscale Spatial Organization of Cell-Adhesive Ligands Direct Stem Cell Fate. Nano Lett. 2015, 15(7), 4720-4729. DOI: 10.1021/acs.nanolett.5b01619.

56. Lee, J.H.; Khang, G.; Lee, J.W.; Lee, H.B. Interaction of Different Types of Cells on Polymer Surfaces with Wettability Gradient. J. Colloid Interface Sci. 1998, 205(2), 323-330. DOI: 10.1006/jcis.1998.5688.

57. Lampin, M.; Warocquier, C.; Legris, C.; Degrange, M.; Sigot-Luizard, M.F. Correlation between substratum roughness and wettability, cell adhesion, and cell migration. J. Biomed. Mater. Res. 1997, 36, 99-108. DOI: 10.1002/(SICI)10974636(199707)36:1,99::AID-JBM12.3.0.CO;2-E.

58. Dowling, D.P.; Miller, I.S.; Ardhaoui, M.; Gallagher, W.M. Effect of surface wettability and topography on the adhesion of osteosarcoma cells on plasmamodified polystyrene. J. Biomater. Appl. 2011, 26(3), 327-347. DOI: 10.1177/0885328210372148.

59. Kunzler T.P.; Drobek T.; Schuler M.; Spencer N.D. Systematic study of osteoblast and fibroblast response to roughness by means of surface-morphology gradients. Biomaterials. 2007, 28, 2175-2182. DOI: 10. 1016/j.biomaterials.2007.01.019.

60. Lange, R.; Luthen, F.; Beck, U.; Rychly, J.; Baumann, A.; Nebe, B. Cellextracellular matrix interaction and physico-chemical characteristics of titanium surfaces depend on the roughness of the material. Biomol. Eng. 2002, 19, 255-261. DOI: 10.1016/S1389- 0344(02)00047-3.

61. Wala, J.; Maji, D.; Das, S. Influence of physico-mechanical properties of elastomeric material for different cell growth. Biomed. Mater. 2017, 12(6), 065002. DOI: 10.1088/1748-605X/aa7e81.

62. Premnath, P.; Tavangar, A.; Tan, B.; Venkatakrishnan, K. Tuning cell adhesion by direct nanostructuring silicon into cell repulsive/adhesive patterns. Exp. Cell Res. 2015, 337(1), 44-52. DOI: 10.1016/j.yexcr.2015.07.028.

63. Wen, J.H.; Vincent, L.G.; Fuhrmann, A.; Choi, Y.S.; Hribar, K.C.; Taylor-Weiner, H.; Chen, S.; Engler, A.J. Interplay of matrix stiffness and protein tethering in stem cell differentiation. Nat. Mater. 2014, 13(10), 979-987. DOI: 10.1038/nmat4051. 


\section{- Chapter 2 -}

Flat and microstructured polymeric membranes in organs-on-chips

64. Engler, A.J.; Sen, S.; Sweeney, H.L.; Discher, D.E. Matrix elasticity directs stem cell lineage specification. Cell. 2006, 126(4), 677-689. DOI: 10.1016/j.cell.2006.06.044.

65. Hulsman, M.; Hulshof, F.; Unadkat, H.; Papenburg, B.J.; Stamatialis, D.F.; Truckenmüller, R.; van Blitterswijk, C.; de Boer, J.; Reinders, M.J. Analysis of high-throughput screening reveals the effect of surface topographies on cellular morphology. Acta Biomater. 2015, 15, 29-38. DOI: 10.1016/j.actbio.2014.12.019.

66. Reimer, A.; Vasilevich, A.; Hulshof, F.; Viswanathan, P.; van Blitterswijk, C.A.; de Boer, J.; Watt, F.M. Scalable topographies to support proliferation and Oct4 expression by human induced pluripotent stem cells. Sci. Rep. 2016, 6, 18948. DOI: $10.1038 /$ srep18948.

67. Ochsner, M.; Dusseiller, M.R.; Grandin, H.M.; Luna-Morris, S.; Textor, M.; Vogel, V.; Smith, M.L. Micro-well arrays for 3D shape control and high resolution analysis of single cells. Lab Chip. 2007, 7(8), 1074-1077. DOI: 10.1039/b704449f.

68. Chen, C.S.; Mrksich, M.; Huang, S.; Whitesides, G.M.; Ingber, D.E. Geometric control of cell life and death. Science. 1997, 276(5317), 1425-1428. DOI: 10.1126/science.276.5317.1425.

69. Lee, J.; Abdeen, A.A.; Wycislo, K.L.; Fan, T.M.; Kilian, K.A. Interfacial geometry dictates cancer cell tumorigenicity. Nat. Mater. 2016, 15(8), 856-862. DOI: 10.1038/nmat4610.

70. Nelson, C.M.; Jean, R.P.; Tan, J.L.; Liu, W.F.; Sniadecki, N.J.; Spector, A.A.; Chen, C.S. Emergent patterns of growth controlled by multicellular form and mechanics. Proc. Natl Acad. Sci. USA. 2005, 102(33), 11594-11599. DOI: 10.1073/pnas.0502575102.

71. Kilian, K.A.; Bugarija, B.; Lahn, B.T.; Mrksich, M. Geometric cues for directing the differentiation of mesenchymal stem cells. Proc. Natl Acad. Sci. USA. 2010, 107(11), 4872-4877. DOI: 10.1073/pnas.0903269107.

72. Papenburg, B.J.; Schüller-Ravoo, S.; Bolhuis-Versteeg, L.A.; Hartsuiker, L.; Grijpma, D.W.; Feijen, J.; Wessling, M.; Stamatialis, D. Designing porosity and topography of poly(1,3-trimethylene carbonate) scaffolds. Acta Biomater. 2009, 5(9), 3281-3294. DOI: 10.1016/j.actbio.2009.05.017.

73. Papenburg, B.J.; Vogelaar, L.; Bolhuis-Versteeg, L.A.; Lammertink, R.G.; Stamatialis, D.; Wessling, M. One-step fabrication of porous micropatterned scaffolds to control cell behavior. Biomaterials. 2007, 28(11), 1998-2009. DOI: 10.1016/j.biomaterials.2006.12.023.

74. Guillemette, M.D.; Cui, B.; Roy, E.; Gauvin, R.; Giasson, C.J.; Esch, M.B.; Carrier, P.; Deschambeault, A.; Dumoulin, M.; Toner, M.; Germain, L.; Veres, T.; Auger, F.A. Surface topography induces 3D self-orientation of cells and extracellular matrix resulting in improved tissue function. Integr. Biol. 2009, 1(2), 196-204. DOI: $10.1039 / \mathrm{b} 820208 \mathrm{~g}$.

75. Soscia, D.A.; Sequeira, S.J.; Schramm, R.A.; Jayarathanam, K.; Cantara, S.I.; Larsen, M.; Castracane, J. Salivary gland cell differentiation and organization on micropatterned PLGA nanofiber craters. Biomaterials. 2013, 34(28), 6773-6784. DOI: 10.1016/j.biomaterials.2013.05.061. 


\section{- Chapter 2 -}

Flat and microstructured polymeric membranes in organs-on-chips

76. Esch, M.B.; Post, D.J.; Shuler, M.L.; Stokol, T. Characterization of in vitro endothelial linings grown within microfluidic channels. Tissue Eng. Part A. 2011, 17(23-24), 2965-2971. DOI: 10.1089/ten.tea.2010.0371.

77. Rumpler, M.; Woesz, A.; Dunlop, J.W.; van Dongen, J.T.; Fratzl, P. The effect of geometry on three-dimensional tissue growth. J. R. Soc. Interface. 2008, 5(27), 1173-1180. DOI: 10.1098/rsif.2008.0064.

78. Hebeiss, I.; Truckenmüller, R.; Giselbrecht, S.; Schepers, U. Novel threedimensional Boyden chamber system for studying transendothelial transport. Lab Chip. 2012, 12(4), 829-834. DOI: 10.1039/c2lc20733h.

79. Yamashita, T.; Kollmannsberger, P.; Mawatari, K.; Kitamori, T.; Vogel, V. Cell sheet mechanics: How geometrical constraints induce the detachment of cell sheets from concave surfaces. Acta Biomater. 2016, 45, 85-97. DOI: 10.1016/j.actbio.2016.08.044.

80. Broaders, K.E.; Cerchiari, A.E.; Gartner, Z.J. Coupling between apical tension and basal adhesion allow epithelia to collectively sense and respond to substrate topography over long distances. Integr. Biol. 2015, 7(12), 1611-1621. DOI: 10.1039/c5ib00240k.

81. He, Z.K.; Ma, M.; Lan, X.R.; Chen, F.; Wang, K.; Deng, H.; Zhang, Q.; Fu, Q. Fabrication of a transparent superamphiphobic coating with improved stability. Soft Matter. 2011, 7, 6435-6443. DOI: 10.1039/ c1sm05574g)

82. Lee, J.H.; Lee, S.J.; Khang, G.; Lee, H.B. Interaction of fibroblasts on polycarbonate membrane surfaces with different micropore sizes and hydrophilicity. J. Biomater. Sci. Polym. Ed. 1999, 10(3), 283-294. DOI: 10.1163/156856299x00351.

83. Gotoh, K.; Yasukawa, A.; Taniguchi, K. Water contact angles on poly(ethylene terephthalate) film exposed to atmospheric pressure plasma. J. Adhes. Sci. Technol. 2011, 25, 307-322. DOI: 10.1163/016942410X511114.

84. Koo, G-H.; Jang, J. Surface modification of poly(lactic acid) by UV/ozone irradiation. Fibers Polym. 2008, 9, 674-678. DOI: 10.1007/s12221-008- 0106-1.

85. Pêgo, A.P.; Poot, A.A.; Grijpma, D.W.; Feijen, J. Copolymers of trimethylene carbonate and epsilon-caprolactone for porous nerve guides: synthesis and properties. J. Biomater. Sci. Polym. Ed. 2001, 12(1), 35-53. DOI: 10.1163/156856201744434.

86. Janvikul, W.; Uppanan, P.; Thavornyutikarn, B.; Kosorn, W.; Kaewkong, P. Effects of surface topography, hydrophilicity and chemistry of surface-treated PCL scaffolds on chondrocyte infiltration and ECM production. Procedia Eng. 2013, 59, 158-165. DOI: 10.1016/j.proeng.2013.05.106

87. Toepke, M.W.; Beebe, D.J. PDMS absorption of small molecules and consequences in microfluidic applications. Lab Chip. 2006, 6(12), 1484-1486. DOI: $10.1039 / \mathrm{b} 612140 \mathrm{c}$.

88. van Meer, B.J.; de Vries, H.; Firth, K.; van Weerd, J.; Tertoolen, L.; Karperien, H.; Jonkheijm, P.; Denning, C.; IJzerman, A.P.; Mummery, C.L. Small molecule absorption by PDMS in the context of drug response bioassays. Biochem. Biophys. Res. Commun. 2017, 482(2), 323-328. DOI: 10.1016/j.bbrc.2016.11.062. 


\section{- Chapter 2 -}

Flat and microstructured polymeric membranes in organs-on-chips

89. Giselbrecht, S.; Gietzelt, T.; Gottwald, E.; Guber, A.E., Trautmann, C.; Truckenmuller, R., Weibezahn, K.F. Microthermoforming as a novel technique for manufacturing scaffolds in tissue engineering (CellChips). IEE Proc. Nanobiotechnol. 2004, 151, 151-157. DOI: 10.1049/ip-nbt:20040824)

90. Giselbrecht, S.; Gietzelt, T.; Gottwald, E.; Trautmann, C.; Truckenmüller, R.; Weibezahn, K.F.; Welle, A. 3D tissue culture substrates produced by microthermoforming of pre-processed polymer films. Biomed. Microdevices. 2006, 8(3), 191-199. DOI: 10.1007/s10544-006-8174-8.

91. Truckenmüller, R.; Giselbrecht, S.; van Blitterswijk, C.; Dambrowsky, N.; Gottwald, E.; Mappes, T.; Rolletschek, A.; Saile, V.; Trautmann, C.; Weibezahn, K.F.; Welle, A. Flexible fluidic microchips based on thermoformed and locally modified thin polymer films. Lab Chip. 2008, 8(9), 1570-1579. DOI: 10.1039/b803619e.

92. Vozzi, G.; Flaim, C.; Ahluwalia, A.; Bhatia, S. Fabrication of PLGA scaffolds using soft lithography and microsyringe deposition. Biomaterials. 2003, 24, 2533-2540. DOI: 10.1016/S0142-9612(03)00052-8.

93. Girones, M.; Akbarsyah, I.J.; Nijdam, W.; van Rijn, C.J.M.; Jansen, H.V.; Lammertink, R.G.H.; Wessling, M. Polymeric microsieves produced by phase separation micromolding. J. Membr. Sci. 2006, 283, 411-424. DOI: 10.1016/j.memsci.2006.07.016.

94. Vogelaar, L.; Lammertink, R.G.; Barsema, J.N.; Nijdam, W.; Bolhuis-Versteeg, L.A.; van Rijn, C.J.; Wessling, M. Phase separation micromolding: a new generic approach for microstructuring various materials. Small. 2005, 1(6), 645-655. DOI: 10.1002/smll.200400128.

95. Buitinga, M.; Truckenmüller, R.; Engelse, M.A.; Moroni, L.; Ten Hoopen, H.W.; van Blitterswijk, C.A.; de Koning, E.J.; van Apeldoorn, A.A.; Karperien, M. Microwell scaffolds for the extrahepatic transplantation of islets of Langerhans. PloS ONE. 2013, 8(5), e64772. DOI: 10.1371/journal.pone.0064772.

96. Hou, Q.P.; Grijpma, D.W.; Feijen J. Preparation of porous poly(epsiloncaprolactone) structures. Macromol. Rapid Commun. 2002, 23, 247-252. DOI: 10.1002/1521-3927(20020301)23:4,247::AIDMARC247.3.0. CO;2-5.

97. Wallace, G.G.; Higgins, M.J.; Moulton, S.E.; Wang, C. Nanobionics: the impact of nanotechnology on implantable medical bionic devices. Nanoscale. 2012, 4(15), 4327-4347. DOI: 10.1039/c2nr30758h.

98. Rnjak-Kovacina, J.; Weiss, A.S. Increasing the pore size of electrospun scaffolds. Tissue Eng. Part B Rev. 2011, 17(5), 365-372. DOI: 10.1089/ten.teb.2011.0235.

99. Soliman, S.; Sant, S.; Nichol, J.W.; Khabiry, M.; Traversa, E.; Khademhosseini, A. Controlling the porosity of fibrous scaffolds by modulating the fiber diameter and packing density. J. Biomed. Mater. Res. A. 2011, 96(3), 566-574. DOI: 10.1002/jbm.a.33010.

100. Cheng, Q.; Lee, B.L.; Komvopoulos, K.; Li, S. Engineering the microstructure of electrospun fibrous scaffolds by microtopography. Biomacromolecules. 2013, 14(5), 1349-1360. DOI: 10.1021/bm302000n. 
- Chapter 2 -

Flat and microstructured polymeric membranes in organs-on-chips 
- Chapter 2 -

Flat and microstructured polymeric membranes in organs-on-chips 


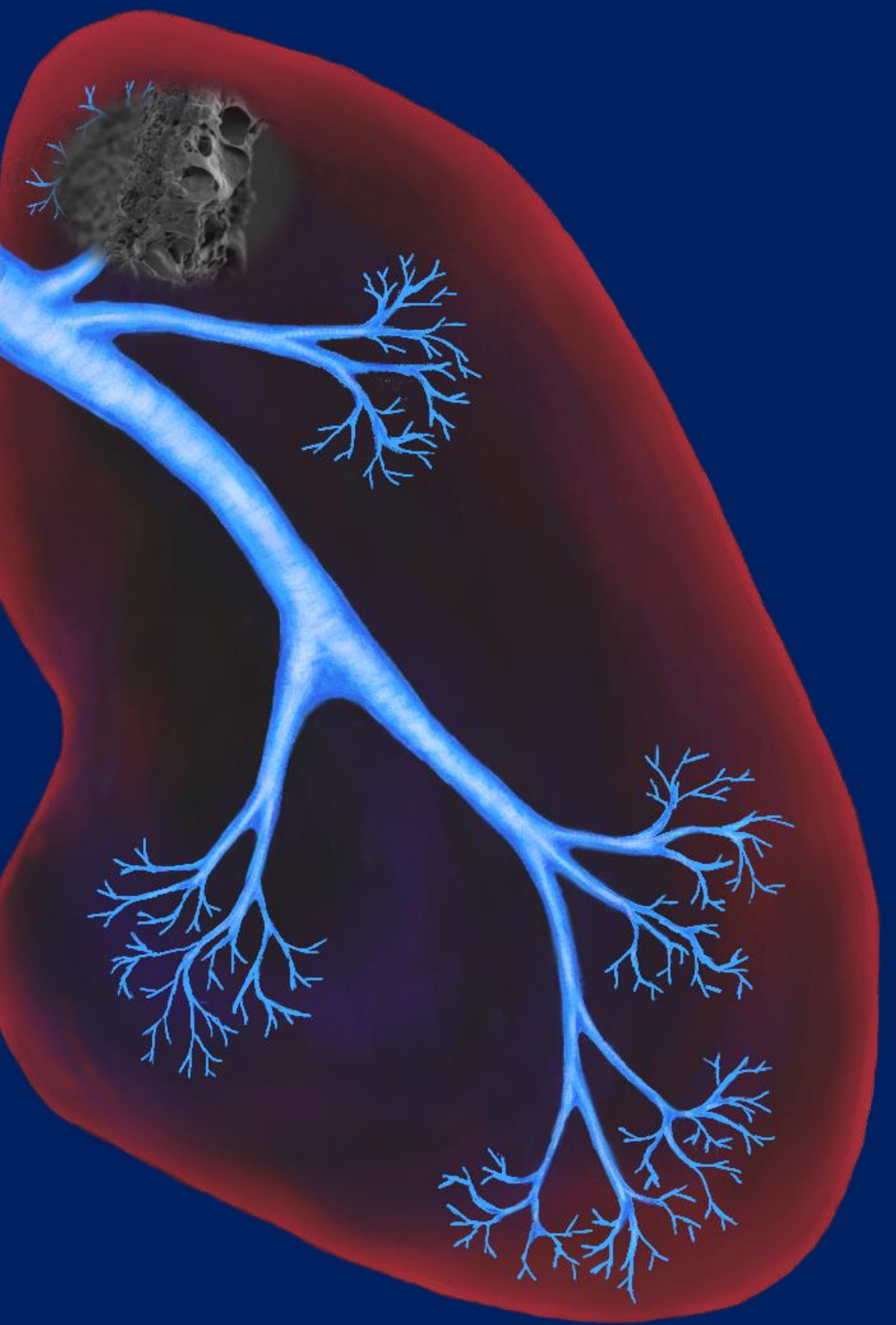




\section{- Chapter 3 -}

\section{Fabricating porous, photo-crosslinked poly(trimethylene carbonate) membranes using temperature-induced phase separation}

Thijs Pasman ${ }^{1}$, Dirk W. Grijpma ${ }^{1,2}$, Dimitrios Stamatialis ${ }^{1}$ and André A. Poot ${ }^{1}$

1. Technical Medical (TechMed) Centre, Department of Biomaterials Science and Technology, Faculty of Science and Technology, University of Twente, 7522 NB Enschede, The Netherlands

2. Department of Biomedical Engineering, Universitair Medisch Centrum Groningen, 9700 AB Groningen, The Netherlands

This chapter was adapted from: Pasman, T.; Grijpma, D.; Stamatialis, D.; Poot, A. Fabricating porous, photo-crosslinked poly(trimethylene carbonate) membranes using temperature-induced phase separation. Polymers for Advanced Technologies. 2017, 28(10), 1258-1262. DOI: 10.1002/pat.3956. 
Fabricating porous, photo-crosslinked PTMC membranes using TIPS

\section{Abstract}

The recent development of lungs-on-chips is a major advancement in lung disease research. However, the materials used for the membranes in these chips, e.g. poly(dimethyl siloxane) and silicon, are not ideal. This study uses the more biocompatible and mechanically favourable polymer poly(trimethylene carbonate) (PTMC). Porous membranes were made of high molecular weight linear PTMC $(250,000 \mathrm{~g} / \mathrm{mol})$ via temperatureinduced phase separation with ethylene carbonate, followed by ultraviolet (UV)-light crosslinking with pentaerythritol triacrylate and Irgacure 2959. Membrane morphology and crosslinking efficacy was investigated. Membranes with micrometre-sized pores could be made. There was variation between the different membrane sides in terms of pore size and distribution. Cooling during UV-light crosslinking resulted in more homogeneous membrane pores, both in size and distribution, and raised the gel content from $48 \%$ to $73 \%$, which can be further enhanced by adjustment of the UV treatment or the amount of crosslinking agents. All membranes were highly permeable to water suggesting that nutrient transport, when used with cells, is not limited by the membranes. Here, we show the development of thin and porous PTMC membranes for lungs-on-chips, Transwell $®$ inserts and other cell culturing applications.

Keywords: PTMC; membranes; TIPS; ethylene carbonate; photo-crosslinking

\section{Introduction}

In recent years, lungs-on-chips with micro-engineered alveolar tissue were developed to accommodate the need for better in vitro models in lung disease research and to combat the limited amount of donor tissue. The first of these chips [1] led to the rise of the field of organs-on-chips, which has seen much progress since then, though current lungs-on-chips still share many similarities with older versions. Lung cells are cultured on one side of a porous support membrane, and in some cases, endothelial cells are cultured on the opposite side as well. The lung cells can be exposed to air, while nutrients reach the cells from the other side of the 
Fabricating porous, photo-crosslinked PTMC membranes using TIPS

membrane. Airflow and nutrients are generally applied to the cells by means of microfluidics. Moreover, the membrane can often be stretched, which causes the cells to experience mechanical stress.

Cells react to material properties, such as stiffness and topography [2-4], thus making the choice of the material for the membrane very significant. In most chips, porous membranes made of polymers like poly(dimethyl siloxane) (PDMS) [1,5] or materials such as silicon [6] are used. Those are easy to process but have suboptimal biocompatibility and/or mechanical properties. Generally, a coating with, for example, extracellular matrix components is needed to properly attach and grow cells on these materials.

Therefore, we propose a porous membrane made of poly(trimethylene carbonate) (PTMC). PTMC is a flexible and elastic polymer, which degrades via surface erosion and thus keeps its mechanical properties until it is almost completely degraded. Additionally, the degradation products are nonacidic and nontoxic to cells [7,8]. Results from other studies indicate that PTMC can meet the requirements necessary for a lung-on-a-chip in terms of biocompatibility and mechanical properties [2,9-12].

Moreover, cells attach and grow on PTMC without coatings [2,9-12], which can be an advantage. In fact, after initial cell adhesion, cells are able to create their own extracellular matrix (ECM), and they can adjust the $\mathrm{ECM}$, in terms of orientation and constitution, specifically to the stimuli provided by the chip. The membranes should be porous for nutrient transport and white blood cell (WBC) migration. Commercial assays for WBC migration generally have pores of $3 \mu \mathrm{m}$, whereas lung-on-a-chip membranes contain pores of $8-10 \mu \mathrm{m}$ for similar purposes of lung cell culturing and WBC migration $[1,5,6]$. Based on this, a suitable pore size would be 3-8 $\mu \mathrm{m}$, to limit the migration of cells other than WBCs.

Porous structures can be produced by different techniques. For PTMC, several phase separation techniques have been applied, among others. Papenburg et al. [2] fabricated porous PTMC scaffolds with microstructures by means of phase separation micromoulding (PS $\mu \mathrm{M})$, which is based on liquid-induced phase separation (LIPS). A polymer 
Fabricating porous, photo-crosslinked PTMC membranes using TIPS

solution of PTMC was cast on a substrate and subsequently placed in a non-solvent. Exchange of solvent and non-solvent led to polymer solidification and creation of pores with sizes in a similar range as the ideal pore size for the present study. The stability of the pores during cell culturing was only investigated for a short term. When kept cold and incubated in cell culture medium, pore stability could be enhanced for a short period. However, there were indications that the pores were unstable if kept for longer periods of time. Therefore, it is unlikely that the pores will be stable in the long term, especially under cyclic stretch in a lung-on-a-chip.

Other research employed temperature-induced phase separation (TIPS) for the production of porous PTMC scaffolds [11,13]. PTMC was dissolved in ethylene carbonate (EC) after it was heated to above its melting point of around $37{ }^{\circ} \mathrm{C}$. The resulting solution was cast on a substrate. Subsequent quenching of the temperature below the melting point of EC caused EC crystallisation, after which the crystals were extracted with water. The kinetics and thermodynamics during cooling of the solution affect the crystallisation of the solvent. The faster the temperature is quenched, the faster the solvent crystallises. This leads to smaller pores. By means of TIPS, a wide range of pore sizes can thus be acquired [14].

To preserve the porous structure, the PTMC could be photo-crosslinked into a network through application of ultraviolet (UV) light, a photoinitiator and a source of acrylate groups. The latter can either be present on the PTMC itself [13] or added to the polymer solution as an acrylate such as pentaerythritol triacrylate (PETA) $[8,11]$. UV light creates radicals of the photoinitiator, in turn, forming reactive acrylates and acrylic radicals that abstract hydrogen from the PTMC chains, resulting in network formation. Crosslinking of PTMC yields creep-free networks $[8,11]$, which could be important for the lung-on-a-chip, where the membrane is exposed to cyclic stretch. Therefore, having good mechanical properties to withstand this stress while maintaining its membrane morphology is required. 
Fabricating porous, photo-crosslinked PTMC membranes using TIPS

The aim of this study was to prepare porous and stable PTMC membranes by means of TIPS and photo-crosslinking. This could provide novel biocompatible membranes for use in lungs-on-chips or other (cell culturing) applications.

\section{Materials and methods}

\subsection{Materials}

1,3-trimethylene carbonate (TMC, Boehringer Ingelheim, Germany), stannous octoate (Spectrum chemical MFG corp., USA), pentaerythritol triacrylate (PETA) (Sigma-Aldrich, USA, cat. 246794), 2-Hydroxy-4'-(2hydroxyethoxy)-2-methylpropiophenone (Irgacure 2959) (Sigma-Aldrich, cat. 410896) and ethylene carbonate (EC) (Sigma-Aldrich, cat. E26258) were used as received. MilliQ water (Millipore Advantage A10, Merck Millipore, Germany), chloroform (Merck Millipore, Germany, Emparta cat. 1.07024.2500, analytical grade), ethanol (Merck Millipore, Emsure cat. 1.02445.1000, analytical grade) and deuterated chloroform (SigmaAldrich, cat. 151831) were also used as received.

\subsection{Polymer synthesis}

Poly(trimethylene carbonate) was synthesised by ring-opening polymerisation of TMC under vacuum for 3 days at $130^{\circ} \mathrm{C}$ using stannous octoate as catalyst (9.6 wt \%) and MilliQ water (4.8 wt\%) as initiator. The polymer was dissolved in chloroform and afterwards precipitated in a five-fold volume amount of ethanol for purification. The polymer was then dried at $\pm 20^{\circ} \mathrm{C}$ under vacuum until constant weight. Monomer conversion was determined by proton nuclear magnetic resonance spectroscopy (Bruker, Ascend $400 \mathrm{MHz}$ ) (Billerica, USA) using deuterated chloroform as a solvent. Gel permeation chromatography (GPC, Viscotek GPC Max VE 2001 \& Viscotek TDA 302) (Malvern Instruments, Malvern, United Kingdom) was used to determine the average molecular weight (MW). 
Fabricating porous, photo-crosslinked PTMC membranes using TIPS

\subsection{Membrane fabrication}

Ethylene carbonate was heated to a liquid state at $50{ }^{\circ} \mathrm{C}$ after which PTMC was added ( $3 \mathrm{wt} \%)$. In addition, PETA and the photoinitiator Irgacure 2959 (10 and $5 \mathrm{wt} \%$, respectively, both relative to the PTMC) were added to the solution. The solution was then mixed at $50{ }^{\circ} \mathrm{C}$ for 6 days, after which $500-\mu \mathrm{m}$-thick films were cast on a glass plate with a casting knife. The films were immediately kept in a freezer at $-25{ }^{\circ} \mathrm{C}$ for $60 \mathrm{~min}$. The membranes were then photo-crosslinked by exposure to UV light in a UV machine at $254 \mathrm{~nm}$ (UltraLum crosslinking cabinet, Canada, CEX 1500) for $2 \mathrm{hr}$. This was performed under nitrogen at a distance of $3 \mathrm{~cm}$ from the light source at a light intensity of $5 \mathrm{~mW} / \mathrm{cm}^{2}$. In some cases, the nitrogen gas, which flows into the UV machine, was cooled by passing it through a cold trap immersed in liquid nitrogen. After crosslinking, the membranes were put in MilliQ water (45 times weight excess compared with the polymer) for 6 days at room temperature to extract the EC and any non-reacted or non-crosslinked Irgacure, PETA and PTMC. Water was refreshed once a day. Membranes were then dried until constant weight and stored at $-25^{\circ} \mathrm{C}$.

\subsection{Gel content}

Dry membrane samples were immersed for 2 days in a 150 times weight excess of chloroform, which is a solvent for the non-crosslinked PTMC. The mass of dry samples before (M1) and after (M2) this treatment was measured, and gel content was determined as follows:

$$
\text { Gel content }=\frac{\mathrm{M} 2 \cdot 100}{\mathrm{M} 1}(100 \%)
$$

Data is expressed as mean $\pm \mathrm{SD}$. 
Fabricating porous, photo-crosslinked PTMC membranes using TIPS

\subsection{Scanning Electron Microscopy}

After drying, membrane samples were prepared for scanning electron microscopy (SEM) by gold sputtering in a Cressington sputter coater 108 Auto with a pure gold target (Aurion, The Netherlands, cat. 91017-AU) at $10 \mathrm{~mA}$ for $60 \mathrm{~s}$. SEM imaging was performed on a JSM-6010LA from JEOL (Tokyo, Japan) at $5 \mathrm{kV}$. Image analysis (with Image J) was used to estimate the surface porosity and pore size. Data is expressed as mean \pm SD. Complete images at 500 times magnification were used for these analyses.

\subsection{Clean water flux}

Circular parts with a diameter of $26 \mathrm{~mm}$ were punched out of the membranes and placed in an Amicon cell (Merck Millipore, Germany, Amicon cell 8003) with a filtration area of $0.9 \mathrm{~cm}^{2}$. MilliQ water was introduced to the membranes under different water pressures. After reaching a stable water flow through the membrane, the amount of water passing through the membrane (permeate) was measured every second for a minimum of $15 \mathrm{~min}$. Data is expressed as mean $\pm \mathrm{SD}$.

\section{Results}

A correct pore size (distribution) and transmembrane transport are important for the present application. Other important characteristics for the membranes are stability of the pores and suitable mechanical properties. Linear high molecular weight PTMC with an MW of 250,000 $\mathrm{g} / \mathrm{mol}$, as determined by GPC, was used to prepare the membranes. In order to fabricate the membranes, solutions were cast, cooled to phase separate, crosslinked with UV light, and washed afterwards. Crosslinking was done in either an uncooled or cooled UV machine. Without cooling, the temperature in the UV machine during UV treatment was approximately $39{ }^{\circ} \mathrm{C}$, which is above the melting point of EC. To avoid melting of the EC, the UV machine was cooled with cold nitrogen gas. Consequently, the maximum temperature in the UV machine decreased to approximately $30^{\circ} \mathrm{C}$, which is below the melting point of EC. 
Fabricating porous, photo-crosslinked PTMC membranes using TIPS

The membranes prepared with the uncooled or cooled UV machine will

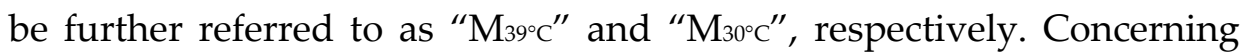
membrane morphology, the membrane thickness was similar for $\mathrm{M}_{39^{\circ} \mathrm{C}}$ and $\mathrm{M}_{30^{\circ} \mathrm{C}}$, as membranes had an average thickness of $50.5 \pm 3.3$ and $55.4 \pm$ $10.8 \mu \mathrm{m}$, respectively.

In both conditions, membranes were porous on the air side (facing the air during casting), the glass side, and throughout the membrane (Figure 1AC). Surface porosity of the membranes differed between the air and glass side but did not change with cooling. $\mathrm{M}_{39^{\circ} \mathrm{C}}$ and $\mathrm{M}_{30^{\circ} \mathrm{C}}$ had a surface porosity on the air side of $8.0 \%$ and $8.3 \%$, respectively, while the porosity on the glass side was $13.1 \%$ and $16.3 \%$, respectively (Figure $1 \mathrm{~A}, \mathrm{~B}$ and Table 1). The average pore diameter was similar on the air and glass side for both $\mathrm{M}_{39^{\circ} \mathrm{C}}$ and $\mathrm{M}_{30^{\circ} \mathrm{C}}$, ranging from 0.9-1.3 $\mu \mathrm{m}$ (Table 1).

The majority of the pores were thus smaller than the desired 3-8 $\mu \mathrm{m}$ described before, though bigger pores were also present, especially in the case of $\mathrm{M}_{39^{\circ} \mathrm{C}}$ (Figure $1 \mathrm{~A}-\mathrm{C}$ ). The air side of the latter membranes was rough, and there were some large pores of up to $40 \mu \mathrm{m}$ in diameter. Crosssection of the membranes showed an interface, where the air side and most of the cross-section had a rough surface with large pores and high variation in pore size. The glass side was smoother, and the maximum pore size amounted to $9.5 \mu \mathrm{m}$ (Figure 1B and Table 1). When kept in a cool UV machine, the maximum pore diameter of membranes became similar on both sides. While the maximum pore diameter increased at the glass side to $14.6 \mu \mathrm{m}$, it decreased on the air side to $15.9 \mu \mathrm{m}$ (Figure 1 and Table 1). 
- Chapter 3 -

Fabricating porous, photo-crosslinked PTMC membranes using TIPS

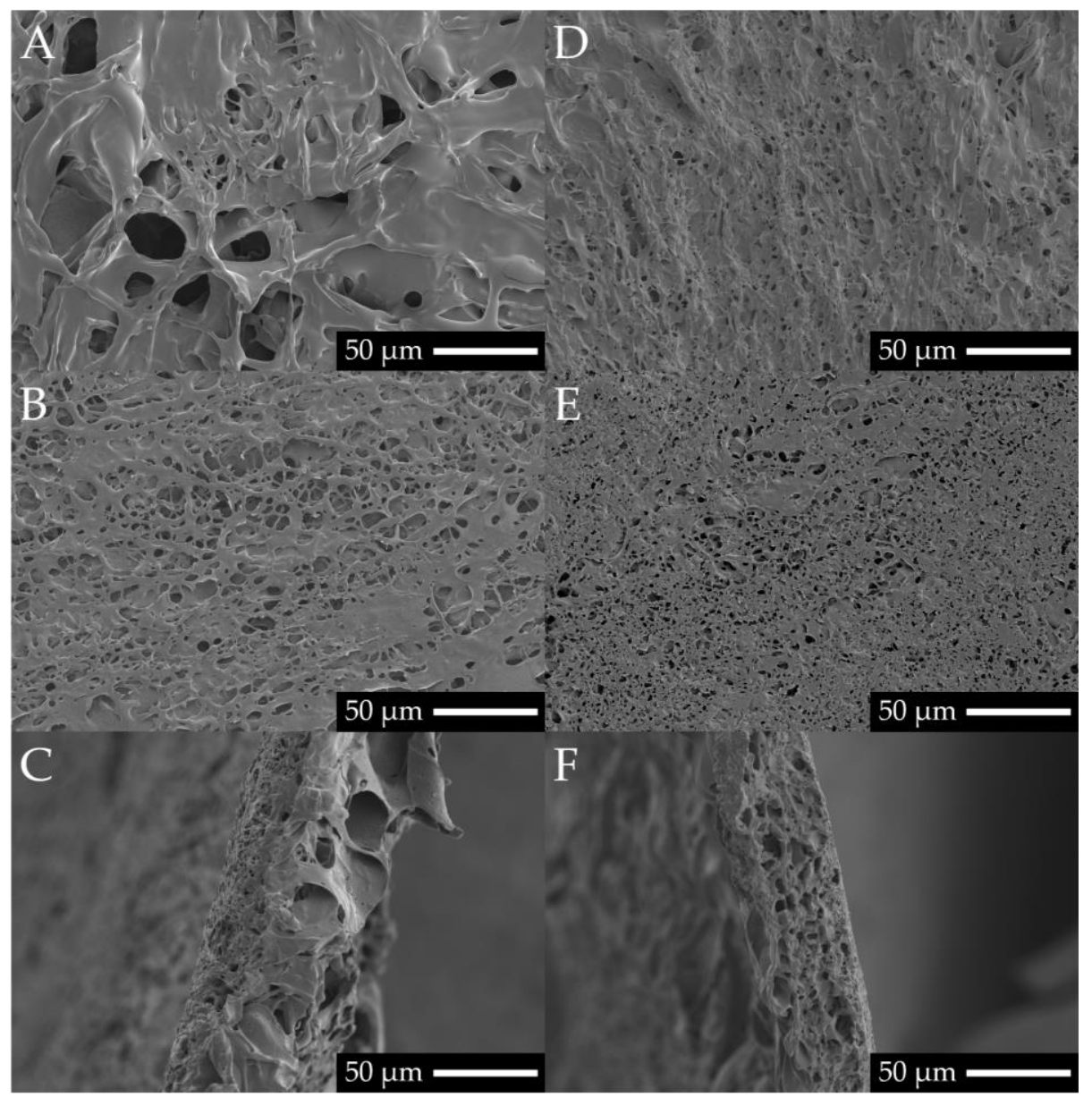

Figure 1. Pore morphology of poly(trimethylene carbonate) membranes made by temperature-induced phase separation. Scanning electron microscopy images of ( $A$ and $D$ ) the air side, ( $B$ and $E$ ) the glass side, and ( $C$ and $F$ ) the cross-section of

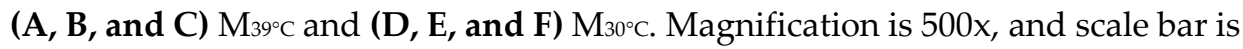
$50 \mu \mathrm{m}$. 
Fabricating porous, photo-crosslinked PTMC membranes using TIPS

Table 1. Pore analysis of poly(trimethylene carbonate) membranes. Analysis of pore diameter, porosity and maximum pore diameter of membranes analysed by ImageJ.

\begin{tabular}{|c|c|cc|cc|c|}
\hline $\begin{array}{c}\text { Temperature during } \\
\text { ultraviolet } \\
\text { treatment }\end{array}$ & Side & Porosity (\%) & $\begin{array}{c}\text { Pore diameter } \\
(\mu \mathrm{m})\end{array}$ & $\begin{array}{c}\text { Maximum } \\
\text { pore } \\
\text { diameter }\end{array}$ \\
\hline $39^{\circ} \mathrm{C}$ & Air & 8.0 & 4.7 & 1.3 & 0.4 & 40.4 \\
\hline $39^{\circ} \mathrm{C}$ & Glass & 13.1 & 1.1 & 0.9 & 0.03 & 9.5 \\
\hline $30^{\circ} \mathrm{C}$ & Air & 8.3 & 1.1 & 1.1 & 0.4 & 15.9 \\
\hline $30^{\circ} \mathrm{C}$ & Glass & 16.3 & 2.1 & 1.0 & 0.2 & 14.6 \\
\hline
\end{tabular}

To retain this pore and membrane morphology, stability of the membrane structure is important. Average gel content of $\mathrm{M}_{39^{\circ} \mathrm{C}}$ was $48 \% \pm 10 \%$, indicating that the membranes partially consisted of a polymer network, which improves the stability of the membrane and its pores. Cooling during UV exposure increased the gel content to $73 \% \pm 9 \%$.

Transmembrane transport is another key aspect of membranes. All membranes were highly permeable to water. Interconnectivity of the pores was investigated by means of water flux experiments (Figure 2). $\mathrm{M}_{39^{\circ} \mathrm{C}}$ had a permeability coefficient of approximately 123,000 $\mathrm{L} /\left(\mathrm{m}^{2} \cdot \mathrm{h} \cdot \mathrm{bar}\right)$. Also without applying transmembrane pressure, there was already a water flux through the membranes (not measured). $\mathrm{M}_{30^{\circ} \mathrm{C}}$ had a lower permeability of approximately $8,400 \mathrm{~L} /\left(\mathrm{m}^{2} \cdot \mathrm{h} \cdot \mathrm{bar}\right)$. In both cases, there was a large variation between membranes (Figure 2). Despite this variation, the flux increased with increasing pressure in an almost linear fashion with a correlation coefficient of 0.99 or higher for both conditions. 


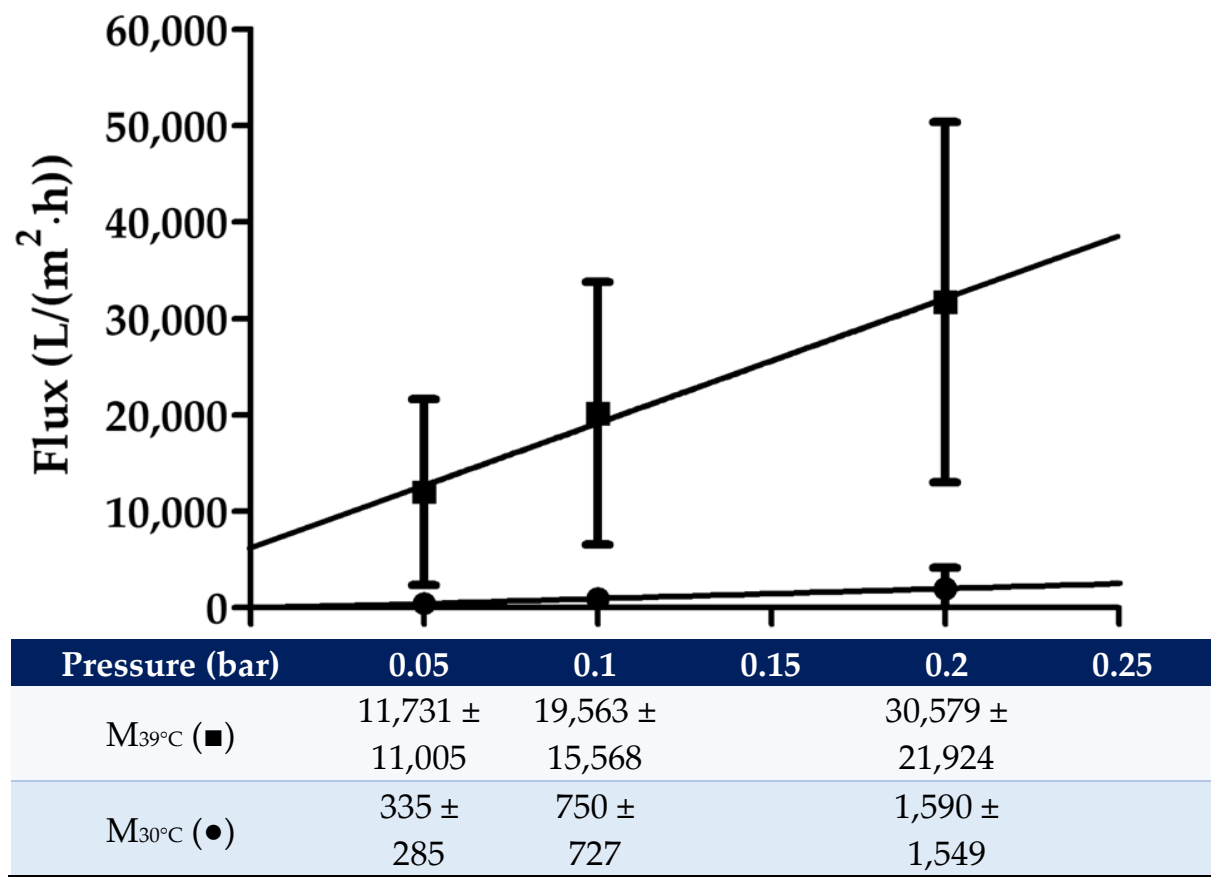

Figure 2. Water flux of membranes. Average flux in litres per hour for a square metre of membrane \pm the standard deviation (SD) are presented for $\mathrm{M}_{39^{\circ} \mathrm{C}}(\mathbf{-})$ and $\mathrm{M}_{30^{\circ} \mathrm{C}}(\bullet)$. Dotted lines represent trend lines.

\section{Discussion}

The basic principle of any membrane relies on a selective separation of ions, molecules, or other particles, either for transport, purification, or other purposes. Thus, a correct pore size (distribution) is very important for the present application, especially the maximum pore size is also of interest. If pores are too large, the lung cells (and endothelial cells) could migrate in or through the membrane, either blocking the pores or creating heterogeneous cell populations in the case of multiple cell types. Cells could also be unable to form a confluent cell layer if the pores are too large for the cells to span.

The average pore diameter of approximately $1 \mu \mathrm{m}$ of all fabricated membranes was smaller than desired. Although this concerns the 
Fabricating porous, photo-crosslinked PTMC membranes using TIPS

majority of the pores, membranes also contained pores in the ideal poresize range of $3-8 \mu \mathrm{m}$ pore size on the glass and air side of the initially prepared membranes was similar. However, the maximum pore diameter varied substantially. On the glass side, the maximum pore diameter exceeded the desired pore diameter (Table 1) but was still below $10 \mu \mathrm{m}$ as used in some lungs-on-chips. The glass side was homogeneous and smooth, with relatively many small pores. The air side, however, was rough and showed both dense areas as well as large pores that far exceeded the desired size.

Cooling the UV machine during UV treatment to approximately $30{ }^{\circ} \mathrm{C}$ resulted in a maximum surface pore size of approximately $15 \mu \mathrm{m}$ on both sides of the membranes (Figure 1 and Table 1). This suggests that the decrease in temperature was enough to keep the EC crystals fixed in place. The surface on the air side was also smoother. Average pore size, porosity, and membrane thickness did not change because of the lower temperature in the UV machine. The structure of the membrane was thus preserved, resulting in a more uniform pore size, which better meets the requirements for the present application (Figure 1 and Table 1).

Previously, Papenburg et al. [2] used poly(ethylene oxide) (PEO) as an additive for membrane fabrication via LIPS. PEO acted as a hydrophilic additive to tailor the membrane phase separation as well as a pore former. In fact, addition of higher amounts of PEO to the membrane polymer dope resulted in increased membrane porosity and average pore size. Using TIPS, however, addition of additives is not needed as different pore sizes can be obtained [11,13] by altering the quenching rate [14]. In our case, a lower quenching rate will increase the pore size.

Crosslinking in the $30{ }^{\circ} \mathrm{C} \mathrm{UV}$ machine resulted in a membrane with better crosslinking as gel content increased to $73 \%$. As the temperature does not reach the melting point of $\mathrm{EC}$ in the $30{ }^{\circ} \mathrm{C} \mathrm{UV}$ machine, the EC crystals stay intact. The crystals most likely keep the polymer and crosslinking agents fixed, which improves the photo-crosslinking. The membrane crosslinking can be further increased by tuning the PETA to Irgacure to PTMC ratios, the irradiation time or other factors as discussed by Bat et al. [11]. This is relevant, as the gel content not only relates to pore stability 
Fabricating porous, photo-crosslinked PTMC membranes using TIPS

but also influences the mechanical properties of the membranes. These are important characteristics for the final application of the membranes in a lung-on-a-chip, where cyclic stretch will be applied to the membranes. Both membranes, crosslinked at $30^{\circ} \mathrm{C}$ or $39^{\circ} \mathrm{C}$, were highly permeable for water. Crosslinking at $30{ }^{\circ} \mathrm{C}$ resulted in a membrane with much lower water permeability than the ones crosslinked at $39^{\circ} \mathrm{C}$. As the average pore size and porosity were similar between the two cases, the difference in permeability is probably due to the presence of a few large pores on the

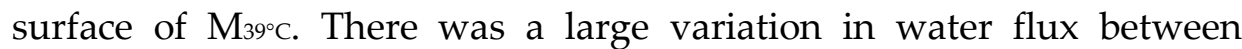
membranes in both cases. Increasing the consistency in structure of the membrane and stability of this structure should reduce the differences between membranes. This could be accomplished by further improvement in the consistency of the membranes' environment, such as temperature, together with increasing the gel content, as mentioned above. Water fluxes showed a strong linear relation between pressure and flux in all membranes (Figure 2). There were no indications of mechanical compaction of the membranes during the filtration experiments within the studied transmembrane pressure range. Membranes crosslinked at 39 ${ }^{\circ} \mathrm{C}$ showed transport of water through the membranes without any external pressure (not measured), as is also suggested by extrapolating the water flux data (Figure 2).

Because for both types of developed membranes the water transport is very high, one would expect that the transport of medium and nutrients to the cells would also be very high.

This study shows the development of thin, porous PTMC membranes by TIPS. It further confirms that temperature is also important after the initial phase separation. Adjusting the quenching rate and crosslinking density while closely regulating the temperature of the membrane during the different fabrication steps should further enhance the pore morphology and stability of these membranes. Because of the flexibility, elasticity as well as biocompatibility of PTMC, these membranes have potential to replace currently used membranes in Transwell ${ }^{\circledR}$ systems and organs-onchips. 


\section{References}

1. Huh, D.; Matthews, B.D.; Mammoto, A.; Montoya-Zavala, M.; Hsin, H.Y.; Ingber, D.E. Reconstituting organ-level lung functions on a chip. Science (New York, N.Y.). 2010, 328(5986), 1662-1668. DOI: 10.1126/science.1188302.

2. Papenburg, B.J.; Schüller-Ravoo, S.; Bolhuis-Versteeg, L.A.; Hartsuiker, L.; Grijpma, D.W.; Feijen, J.; Wessling, M.; Stamatialis, D. Designing porosity and topography of poly(1,3-trimethylene carbonate) scaffolds. Acta biomaterialia. 2009, 5(9), 3281-3294. DOI: 10.1016/j.actbio.2009.05.017.

3. Denning, D.; Roos, W.H. Elucidating the molecular mechanisms underlying cellular response to biophysical cues using synthetic biology approaches. Cell adhesion \& migration. 2016, 10(5), 540-553. DOI: 10.1080/19336918.2016.1170259.

4. Soscia, D.A.; Sequeira, S.J.; Schramm, R.A.; Jayarathanam, K.; Cantara, S.I.; Larsen, M.; Castracane, J. Salivary gland cell differentiation and organization on micropatterned PLGA nanofiber craters. Biomaterials. 2013, 34(28), 6773-6784. DOI: 10.1016/j.biomaterials.2013.05.061.

5. Stucki, A.O.; Stucki, J.D.; Hall, S.R.; Felder, M.; Mermoud, Y.; Schmid, R.A.; Geiser, T.; Guenat, O.T. A lung-on-a-chip array with an integrated bio-inspired respiration mechanism. Lab on a chip. 2015, 15(5), 1302-1310. DOI: 10.1039/c4lc01252f.

6. Punde, T.H.; Wu, W.H.; Lien, P.C.; Chang, Y.L.; Kuo, P.H.; Chang, M.D.; Lee, K.Y.; Huang, C.D.; Kuo, H.P.; Chan, Y.F.; Shih, P.C.; Liu, C.H. A biologically inspired lung-on-a-chip device for the study of protein-induced lung inflammation. Integrative biology : quantitative biosciences from nano to macro. 2015, 7(2), 162-169. DOI: 10.1039/c4ib00239c.

7. Bat, E.; van Kooten, T.G.; Feijen, J.; Grijpma, D.W. Resorbable elastomeric networks prepared by photocrosslinking of high-molecular-weight poly(trimethylene carbonate) with photoinitiators and poly(trimethylene carbonate) macromers as crosslinking aids. Acta biomaterialia. 2011, 7(5), 19391948. DOI: 10.1016/j.actbio.2011.01.010.

8. Bat, E.; van Kooten, T.G.; Feijen, J.; Grijpma, D.W. Crosslinking of trimethylene carbonate and D, L-lactide (co-) polymers by gamma irradiation in the presence of pentaerythritol triacrylate. Macromolecular bioscience. 2011, 11(7), 952-961. DOI: 10.1002/mabi.201100031.

9. Schüller-Ravoo, S.; Zant, E.; Feijen, J.; Grijpma, D. W. Preparation of a designed poly(trimethylene carbonate) microvascular network by stereolithography. Advanced healthcare materials. 2014, 3(12), 2004-2011. DOI: 10.1002/adhm.201400363.

10. Schüller-Ravoo, S.; Teixeira, S.M.; Feijen, J.; Grijpma, D.W.; Poot, A.A. Flexible and elastic scaffolds for cartilage tissue engineering prepared by stereolithography using poly(trimethylene carbonate)-based resins. Macromolecular bioscience. 2013, 13(12), 1711-1719. DOI: 10.1002/mabi.201300399. 


\section{- Chapter 3 -}

Fabricating porous, photo-crosslinked PTMC membranes using TIPS

11. Bat, E.; Kothman, B.H.; Higuera, G.A.; van Blitterswijk, C.A.; Feijen, J.; Grijpma, D.W. Ultraviolet light crosslinking of poly(trimethylene carbonate) for elastomeric tissue engineering scaffolds. Biomaterials. 2010, 31(33), 8696-8705. DOI: 10.1016/j.biomaterials.2010.07.102.

12. Song, Y.; Kamphuis, M.M.; Zhang, Z.; Sterk, L.M.; Vermes, I.; Poot, A.A.; Feijen, J.; Grijpma, D.W. Flexible and elastic porous poly(trimethylene carbonate) structures for use in vascular tissue engineering. Acta biomaterialia. 2010, 6(4), 1269-1277. DOI: 10.1016/j.actbio.2009.10.002.

13. Zant, E.; Blokzijl, M.M.; Grijpma, D.W. A Combinatorial Photocrosslinking Method for the Preparation of Porous Structures with Widely Differing Properties. Macromolecular rapid communications. 2015, 36(21), 1902-1909. DOI: 10.1002/marc.201500229.

14. Mannella, G.A.; Conoscenti, G.; Carfi Pavia, F.; La Carrubba, V.; Brucato, V. Preparation of polymeric foams with a pore size gradient via Thermally Induced Phase Separation (TIPS). Materials Letters. 2015, 160, 31-33. DOI: 10.1016/j.matlet.2015.07.055. 
- Chapter 3 -

Fabricating porous, photo-crosslinked PTMC membranes using TIPS 
- Chapter 3 -

Fabricating porous, photo-crosslinked PTMC membranes using TIPS

$-76-$ 


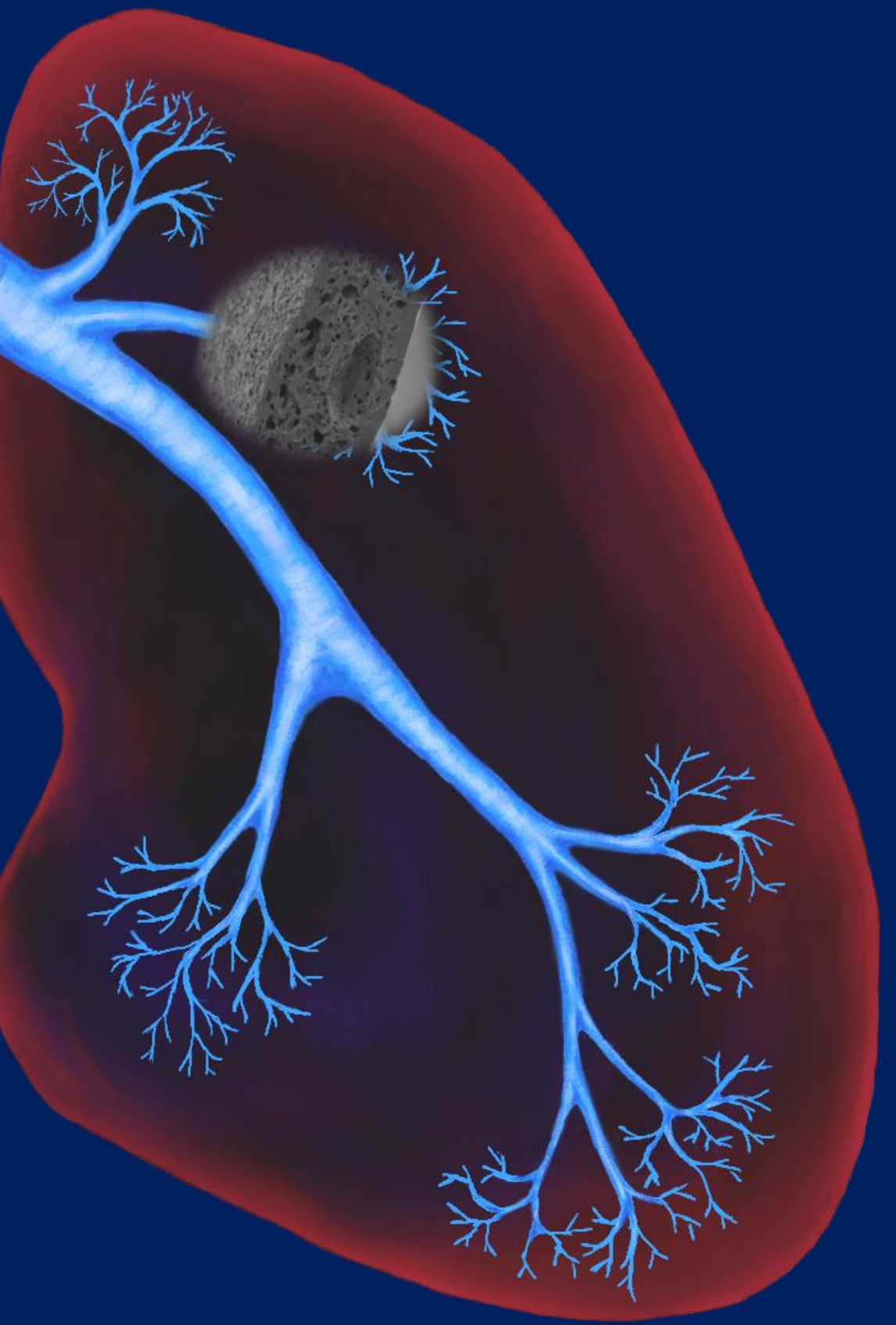




\section{- Chapter 4 -}

\section{Porous and form-stable poly(trimethylene carbonate) membranes prepared by liquid- induced phase separation and photo- crosslinking}

Thijs Pasman, Eef Braam, Dimitrios Stamatialis and André A. Poot

Technical Medical (TechMed) Centre, Department of Biomaterials Science and Technology, Faculty of Science and Technology, University of Twente, 7522 NB Enschede, The Netherlands 
Porous PTMC membranes prepared by LIPS and photo-crosslinking

\section{Abstract}

There is a great demand for biomimetic organ models. However, properties such as the stiffness of most membranes on which cells are grown in these models do not mimic native tissue. Here, we used poly(trimethylene carbonate) (PTMC), which is cytocompatible and mechanically favourable, to fabricate porous membranes by liquidinduced phase separation (LIPS) using poly(ethylene oxide) (PEO) or poly(vinyl pyrrolidone) (PVP) as pore formers. Pore size and porosity increased with increasing molecular weight and/or concentration of the pore formers. To improve the stability of the porous membranes, we photo-crosslinked the membranes by introducing crosslinking agents after LIPS followed by UV-treatment. This study shows for the first time that LIPS and photo-crosslinking can be combined to yield porous and form-stable PTMC membranes with good pore interconnectivity and high water permeance. This crosslinking method enables crosslinking of LIPSbased membranes made from PTMC and possibly other polymers, providing new ways to produce form-stable and porous membranes. However, the reproducibility of these membranes requires further optimisation before they can be applied in organ models.

Keywords: poly(trimethylene carbonate) (PTMC); poly(ethylene oxide) (PEO); poly(vinyl pyrrolidone) (PVP); liquid-induced phase separation (LIPS); photo-crosslinking; membranes

\section{Introduction}

There is a great need for novel biomimetic organ models since the development of new medicines and treatments is becoming more expensive and complex. These organ models should provide more reliable results than traditional models (e.g. static cell cultures on tissue culture plastic) as they would better represent the native tissue. Moreover, they avoid the scientific and ethical problems concerning animal models. 
Porous PTMC membranes prepared by LIPS and photo-crosslinking

Many organ models contain a polymeric membrane on which cells can attach and grow [1]. Membrane properties, such as stiffness, porosity and surface topography, affect cell behaviour [1]. Many current organ models include membranes made from poly(bisphenol-A-carbonate) (PC) or poly(ethylene terephthalate) (PET) [1]. These membranes are transparent and have pores via ion track etching [1]. However, PC and PET have much higher elastic moduli (i.e. 2-2.4 GPa [2] and 2-3 GPa [3], respectively) than most tissues [1,4-7]. Besides PC or PET, many organson-chips (OOCs), small biomimetic in vitro models that introduce physiological stimuli such as fluid flow and mechanical stress to the cells, often contain membranes made from poly(dimethyl siloxane) (PDMS) [1,8-13]. PDMS is easy to use and can have a wide range of elastic moduli [14]. However, PDMS does ad- and absorb small molecules, which complicates (drug) studies, and cell attachment on PDMS is low [15-17].

A different polymer is poly(trimethylene carbonate) (PTMC), which has been used quite extensively for scaffolds, films and porous membranes for biomedical applications $[18,19]$ due to its many suitable characteristics such as good cell adhesive and mechanical properties [18-23]. Previously, we made porous PTMC membranes for the purpose of cell culturing using temperature-induced phase separation (TIPS) [24]. Although these membranes were porous and permeable, their reproducibility was low.

Papenburg et al. used liquid-induced phase separation (LIPS) to develop porous PTMC scaffolds [21]. Moreover, they used LIPS in conjunction with micromoulding to prepare microstructured membranes for tissue engineering applications. The inclusion of microstructures is interesting to better mimic native tissue. They also investigated the effect of adding poly(ethylene oxide) (PEO) to the polymer dope on the membrane pore morphology [21]. PEO can act as a pore former and pore stabiliser [21,25]. However, Papenburg et al. reported a loss of porosity of the membranes over time [21]. In fact, because PTMC is an amorphous polymer with a low glass transition temperature $(\mathrm{Tg})$ of $-17{ }^{\circ} \mathrm{C}[21,26]$, it requires crosslinking to prevent collapsing of pores in porous membranes. 
Porous PTMC membranes prepared by LIPS and photo-crosslinking

Although pore collapse could be prevented by storing the membranes at $-20{ }^{\circ} \mathrm{C}[21]$, this may not be applicable to retain porosity during cell culturing, especially when the membranes are subjected to cyclic stretch.

In this study, we investigate the development of form-stable porous PTMC membranes using linear high molecular weight (MW) PTMC, LIPS and photo-crosslinking. We used PEO and poly(vinyl pyrrolidone) (PVP) as pore formers and pore stabilisers. PVP is a hydrophilic polymer used in many applications, including the production of porous membranes for blood purification [27]. We investigated the effect of the MW and concentration of both PEO and PVP on the membrane formation. To enable membrane photo-crosslinking, we used pentaerythritol triacrylate (PETA) as a crosslinking aid and Irgacure 2959 as a photoinitiator, since other studies have successfully used them to photo-crosslink PTMC scaffolds [28,29]. Moreover, we explored different methods to introduce the crosslinking agents to the membranes.

\section{Materials and methods}

\subsection{Materials}

2-Hydroxy-4'-(2-hydroxyethoxy)-2-methylpropiophenone (Irgacure 2959) (cat. 410896), pentaerythritol triacrylate (PETA) (cat. 246794), poly(ethylene oxide) (PEO) of 35,000 g/mol (cat. 81310) and 5,000,000 $\mathrm{g} / \mathrm{mol}$ (cat. 189472), poly(vinyl pyrrolidone) (PVP) of 360,000 g/mol (cat. 81440) were all acquired from Sigma-Aldrich, St. Louis, MO, USA. Fetal bovine serum (FBS) (cat. 10500-064), Glutamax ${ }^{\mathrm{TM}}$ 100X (cat. 35050-061), and penicillin-streptomycin (P/S) (cat. 15140-122) were purchased from Gibco, Grand Island, NJ, USA. Besides, we purchased 2-Benzyl-2dimethylamino-1-(4-morpholinophenyl)-butanone-1) (Irgacure 369) (Ciba, Switzerland), chloroform (Merck Millipore, Germany, Emparta cat. 1.07024.2500, analytical grade), Eagle's Minimum Essential Medium (EMEM) (Lonza Group Ltd., Basel, Switzerland, cat. BE12-662F), ethanol (Merck Millipore, Emsure cat. 1.02445.1000, analytical grade), stannous 
Porous PTMC membranes prepared by LIPS and photo-crosslinking

octoate (Sn(Oct)2, Spectrum chemical MFG corp., USA) and 1,3trimethylene carbonate (TMC, Boehringer Ingelheim, Germany).

\subsection{Poly(trimethylene carbonate) (PTMC) synthesis}

Synthesis of linear PTMC was done by ring-opening polymerisation of TMC under vacuum at $130{ }^{\circ} \mathrm{C}$ for three days in a silanised, dry glass ampoule. MilliQ water (made by a Millipore Advantage A10, Merck Millipore, Germany) and stannous octoate were added to the TMC as initiator and catalyst, respectively. These were added in a weight ratio of TMC to water of 18,000:1 and TMC to stannous octoate of 9,000:1. After synthesis, the PTMC was dissolved in chloroform and purified by precipitation in a ten-fold volume of cold ethanol. Afterwards, it was dried at $\pm 20^{\circ} \mathrm{C}$ under vacuum until constant weight. The number average molecular weight $(\mathrm{Mn})$ of the PTMC, referred to as $\mathrm{MW}$ in the following, and the polydispersity index (i.e. approximately 350,000 g/mol and 1.39, respectively) were determined by gel permeation chromatography (GPC) in a Viscotek GPC Max VE 2001 with a triple detector array (Viscotek TDA 302).

\subsection{Membrane fabrication}

\subsubsection{Polymer dopes}

Polymer dopes with PEO: All polymer dopes containing PEO were made according to Table 1 . The weight percentages ( $\mathrm{wt} \%)$ are all expressed in relation to the entire polymer dope. PTMC of 350,000 g/mol ( $3 \mathrm{wt} \%$ ) was dissolved in chloroform. Either PEO of $35,000 \mathrm{~g} / \mathrm{mol}(1.0 \mathrm{wt} \%)$ or PEO of $5,000,000 \mathrm{~g} / \mathrm{mol}(0.3$ or $1.0 \mathrm{wt} \%)$ was added as an additive. Moreover, PETA (0.15 wt\%) and Irgacure 2959 (0.075 wt \%) were added as crosslinking agents. The polymer dopes were then sealed and stirred in the dark for at least three days at an ambient temperature of $19-20^{\circ} \mathrm{C}$.

Polymer dopes with PVP: All polymer dopes with PVP consisted of components as depicted in Table 2 . All $w \mathrm{t} \%$ are expressed in relation to the total polymer dope. PTMC $(350,000 \mathrm{~g} / \mathrm{mol}, 3 \mathrm{wt} \%)$ was dissolved in chloroform together with $0.3,1.0,2.0,3.0$ or $6.0 \mathrm{wt} \%$ PVP $(360,000 \mathrm{~g} / \mathrm{mol})$ 
- Chapter 4 -

Porous PTMC membranes prepared by LIPS and photo-crosslinking

as an additive. No crosslinking agents were added. Dopes were sealed and stirred at $19-20^{\circ} \mathrm{C}$ in the dark for at least three days.

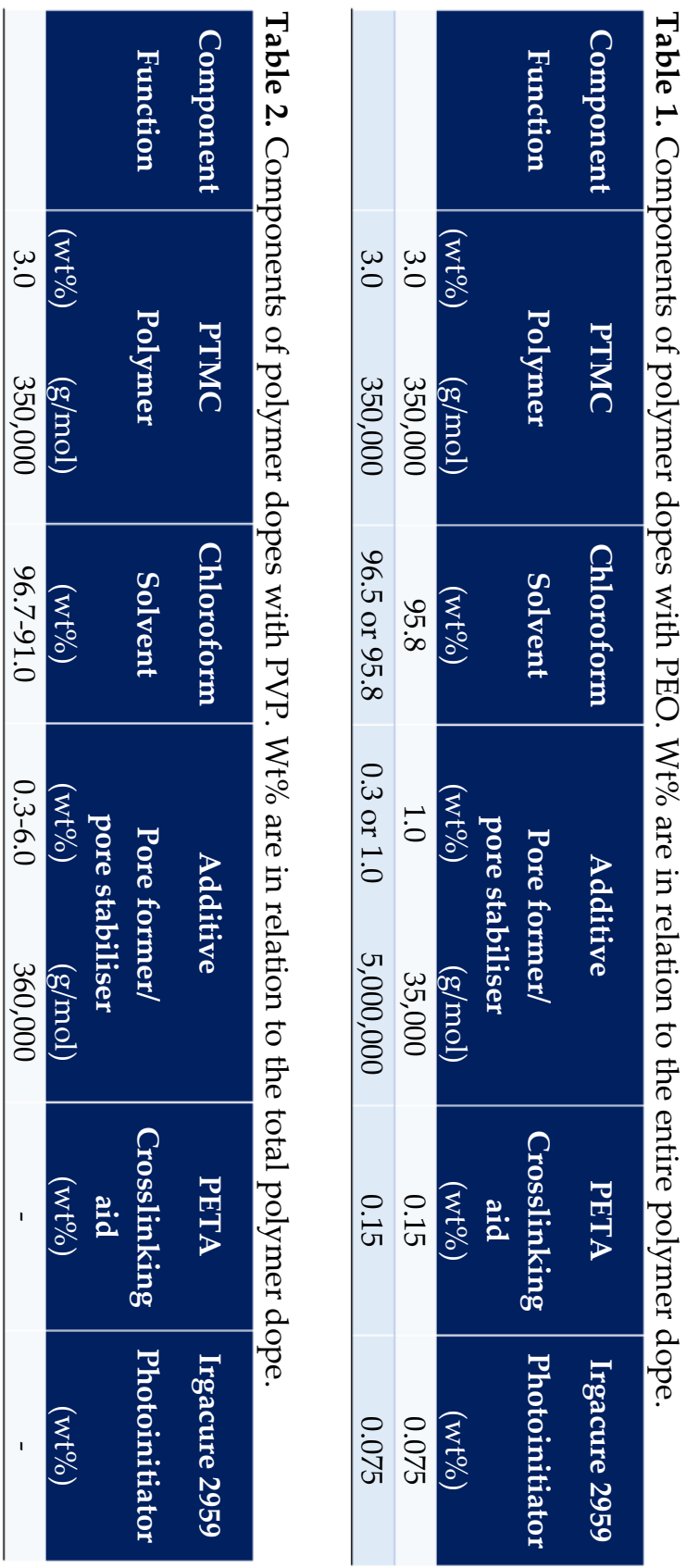




\subsubsection{Casting of membranes}

The polymer dopes were cast on a glass plate in a $500 \mu \mathrm{m}$ thick layer using a casting knife in a fume hood at $19-20{ }^{\circ} \mathrm{C}$. The glass plates and cast polymer dopes and glass plates were immediately submerged in a coagulation bath to commence LIPS. The coagulation bath was sealed and left to stand at $19-20^{\circ} \mathrm{C}$. The coagulation bath consisted of an $8: 1$ volume ratio of ethanol and demi-water and had a volume of 200x the volume of the membrane. LIPS was performed overnight.

Membranes with PEO: After LIPS, all membranes with PEO were treated with UV-light.

Membranes with PVP: After LIPS, the membranes were placed in a small volume of ethanol (i.e. approximately 2.2 times the initial volume of the polymer dope) in a petri dish which approximated the size of the membrane. The ethanol contained $0.6 \mathrm{wt} \%$ PETA and $0.3 \mathrm{wt} \%$ Irgacure 2959. The membranes were incubated in the ethanol with crosslinking agents for approximately two hours to evaporate the ethanol. Afterwards, the membranes were photo-crosslinked using UV-light.

To assess the pore stability of non-crosslinked membranes in cell culture liquids (see Section 2.6), one membrane with $2 \mathrm{wt} \%$ PVP was cast as above but incubated in a small ethanol bath without any crosslinking agents. Subsequently, this membrane was washed immediately instead of receiving UV-treatment.

\subsubsection{Photo-crosslinking with UV-light, washing and drying}

Photo-crosslinking: All membranes which were exposed to crosslinking agents, either in the polymer dope or after LIPS, were crosslinked in the same manner. After LIPS or the small bath with crosslinking agents, membranes were placed on glass plates in a UV-box (UltraLum crosslinking cabinet, Canada, CEX 1500) for two hours at a distance of 3 $\mathrm{cm}$ from the light source. Membranes with Irgacure 2959 were crosslinked at $254 \mathrm{~nm}$ at an intensity of $5 \mathrm{~mW} / \mathrm{cm}^{2}$, while those with Irgacure 369 were crosslinked at $365 \mathrm{~nm}$ and $7 \mathrm{~mW} / \mathrm{cm}^{2}$. Both intensities were measured using an optical power meter (Newport 1916-C, USA). A flow of nitrogen gas of $4 \mathrm{~L} / \mathrm{min}$. was provided through the UV-box to prevent reaction 
between the crosslinking agents and oxygen. Washing: After the UVtreatment, membranes with PEO were washed for three days and membranes with PVP for six days in a large excess of demi-water. The demi-water was replaced every day. The duration of washing was extended for the membranes with PVP to account for the generally larger amounts of PVP present compared to PEO. Drying: After washing, the membranes were rinsed with $100 \%$ ethanol to remove water from the membranes to dry the membranes more efficiently. Membranes were then dried, vacuum sealed and stored at $-20^{\circ} \mathrm{C}$.

\subsection{Membrane morphology}

Scanning Electron Microscopy (SEM) was used to visualise the membrane morphology and assess the pore diameter. Samples of dry membranes were gold-sputtered in a sputter coater (Cressington 108 Auto) outfitted with a pure gold target (Aurion, The Netherlands, cat. 91017-AU) under vacuum at $10 \mathrm{~mA}$ for 60 seconds. A JSM-6010LA microscope from JEOL (Tokyo, Japan) was used to image the samples at $5 \mathrm{kV}$.

\section{$2.5 \mathrm{Gel}$ content of the membranes}

Gel content measurement of membrane samples was performed to determine whether crosslinking was successful. The mass of samples ( $\mathrm{N}$ $\geq 3$ ) of membranes that had been washed and dried was determined (MBefore). The samples were kept in an excess of chloroform for 24 hours. Subsequently, samples were left to dry until constant weight, after which their mass was determined again $\left(\mathrm{M}_{\text {After }}\right)$. Gel content was calculated with Equation 1.

$$
\text { Gel content }=\frac{\mathrm{M}_{\text {After }}}{\mathrm{M}_{\text {Before }}} \cdot 100 \%
$$

\subsection{Membrane morphology in cell culture liquids}

The integrity of the membrane morphology was tested to confirm whether crosslinking had succeeded and if the membranes would remain porous during cell culturing. Circular pieces with a diameter of $15 \mathrm{~mm}$ of 
Porous PTMC membranes prepared by LIPS and photo-crosslinking

membranes were punched, sterilised by incubation in $70 \mathrm{vol} \%$ ethanol for 1 hour and placed in sterile 24-well plates in a sterile laminar fume hood to dry. Afterwards, they were secured to the bottom of the wells with a sterile O-ring. Membranes were then submerged in cell culture medium for two weeks in a cell culture incubator in normal cell culture conditions (i.e. $37^{\circ} \mathrm{C}$ and $5 \% \mathrm{CO}_{2}$ ). The liquids were refreshed three times a week. Morphology of the samples was determined by SEM and compared to that of non-crosslinked samples. Cell culture medium consisted of 88 vol\% EMEM, 10 vol\% FBS, 1 vol\% P/S and 1 vol\% Glutamax.

The membranes used were PTMC membranes with $2 \mathrm{wt} \%$ PVP as an additive, either incubated with crosslinking agents after LIPS before UVtreatment, or not exposed to crosslinking agents and not treated with UVlight (see Section 2.3).

\subsection{Water transport across the membranes}

The membrane pore connectivity was investigated by performing clean water flux experiments for the crosslinked PTMC membranes with $2 \mathrm{wt} \%$ PVP (see Section 2.3). Circular samples $(\mathrm{N}=5)$ with a diameter of $26 \mathrm{~mm}$ were punched from several of these membranes. Following standard protocol, the membranes were pre-wetted for 30 minutes in MilliQ water. Membranes were placed in an Amicon Cell (Merck Millipore, Germany, Amicon Cell 8003) and pre-compacted by applying a transmembrane pressure (TMP) of 1.5 bar until they had a constant water flux. Afterwards, the TMP was lowered, and the amount of permeating water was determined at increasing TMPs of 0.5 bar, 1.0 bar and 1.5 bar. Measurements lasted at least 15 minutes for every TMP. The average permeance of the membranes was determined as the average slope of the best linear fit of the water flux versus the TMP of each sample.

\subsection{Statistical analysis}

Data were analysed using Graphpad Prism5 software. Statistical analysis was performed using a one-way ANOVA and a Bonferroni post-hoc test with $\mathrm{P}<0.05$. Significant differences are marked by capped lines with asterisks. 


\section{Results and discussion}

\subsection{PTMC membranes with PEO}

\subsubsection{PEO as a pore former}

Figure 1 shows the surface morphology of PTMC membranes with PEO. To tailor the porosity and pore size, PEOs with two different MWs were used. The addition of $1 \mathrm{wt} \%$ of $35,000 \mathrm{~g} / \mathrm{mol} \mathrm{PEO}$ to the polymer dope resulted in membranes with very few and relatively small pores compared to membranes made with dopes which contained $1 \mathrm{wt} \%$ of $5,000,000 \mathrm{~g} / \mathrm{mol}$ PEO (Figure 1A-B). It is known that the molecular weight of the additive can influence the phase separation [21]. The pore diameter in the membranes with 5,000,000 g/mol PEO varied greatly from $1 \mu \mathrm{m}$ to more than $20 \mu \mathrm{m}$ (Figure 1). Moreover, many of the pores were in the range of $10-20 \mu \mathrm{m}$ or larger, which is generally considered too large for cell culturing on membranes since most cells would be able to migrate through them. Thus, the amount of PEO was decreased.

Decreasing the amount of 5,000,000 g/mol PEO from $1 \mathrm{wt} \%$ to $0.3 \mathrm{wt} \%$ resulted in membranes with a more homogeneous pore morphology and having lower porosity and pore diameter (i.e. in the range of $1 \mu \mathrm{m}$ to approximately $20 \mu \mathrm{m}$ ) (Figure 1B-C). This is consistent with the effects of PEO reported by Papenburg et al., who used 6,000,000 g/mol PEO to tailor porosity in PTMC scaffolds [21]. The membranes with $0.3 \mathrm{wt} \%$ PEO had similar porosity and pore size on both surfaces of the membranes (i.e. the air side, facing the air during casting, and the substrate side, facing the glass plate on which was cast) (Figure 1C-D). Moreover, the smaller pore diameter, compared to the membranes with $1 \mathrm{wt} \%$, was more suitable for cell culturing, although there were still a considerable number of pores larger than $10 \mu \mathrm{m}$.

Membranes also shrunk during LIPS. Increasing the amount of PEO decreased shrinkage, as was also reported by Papenburg et al. [21]. The membranes were difficult to handle. After phase separation, they were sticky and prone to folding. The former was also found by Papenburg et al. when fabricating microstructured PTMC scaffolds by LIPS with PEO as an additive [21]. They reduced the stickiness by incubating the 
Porous PTMC membranes prepared by LIPS and photo-crosslinking

membranes in cell culture medium. We anticipated that crosslinking of the membranes would reduce the stickiness and folding of the membranes.

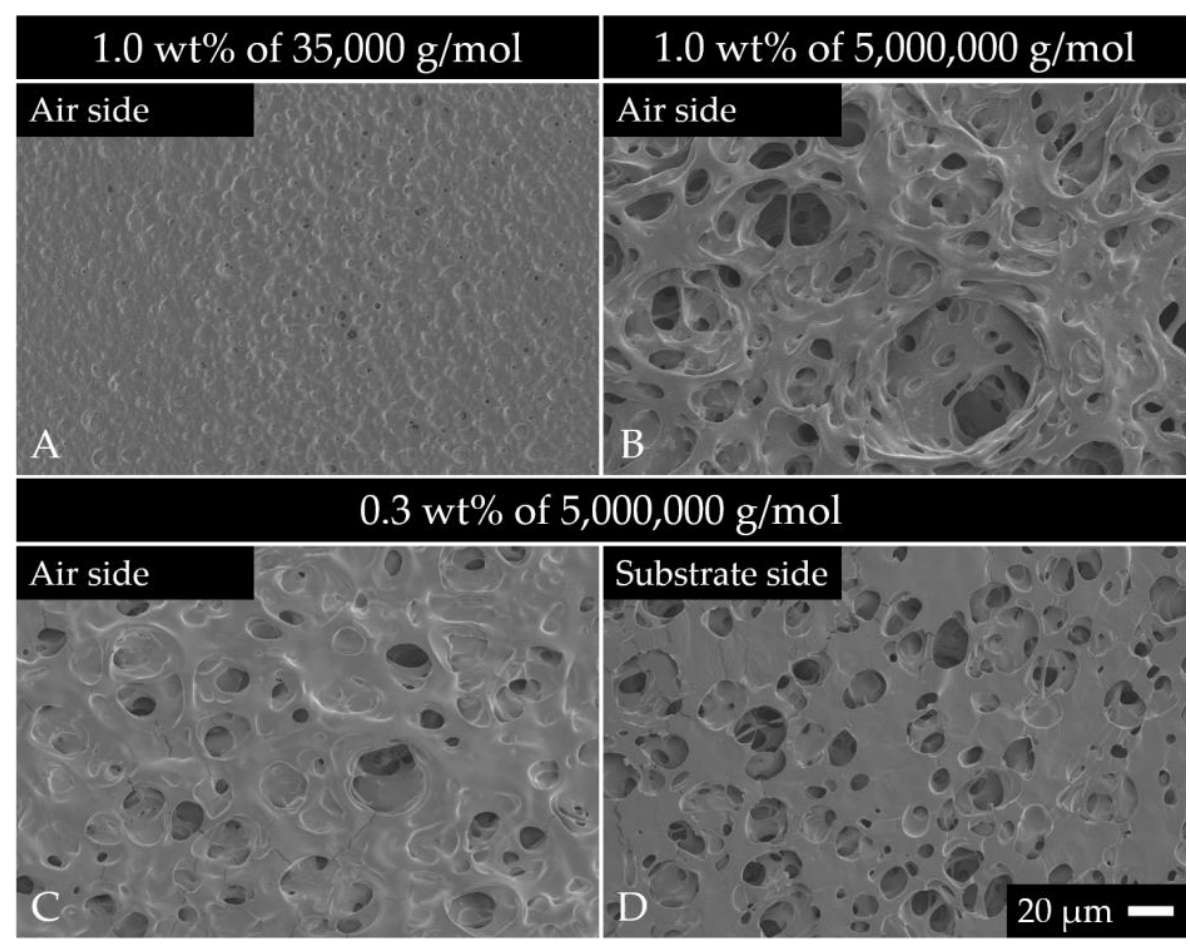

Figure 1. SEM images of membranes made from PTMC with $\mathrm{PEO}$ as an additive. Membranes either contained $1 \mathrm{wt} \%$ PEO of (A) $35,000 \mathrm{~g} / \mathrm{mol}$ or (B) $5,000,000$ $\mathrm{g} / \mathrm{mol}$, or (C-D) $0.3 \mathrm{wt} \%$ PEO of 5,000,000 g/mol. Shown are (A-C) the air side, facing the air during casting, and (D) the substrate side, facing the substrate (i.e. glass plate) during casting. Magnification: 500x. Scale bar applies to all images. Images are representative of observations of multiple samples $(\mathrm{N}=2)$. 


\subsubsection{Crosslinking of the membranes}

The membranes with PEO were treated with UV-light after LIPS to photocrosslink the membranes and obtain stable porous membranes. However, the crosslinking of these membranes was not optimal as indicated by the very low gel content of these membranes (data not shown). Probably, this was caused by a loss of crosslinking agents from the membranes upon submersion in the coagulation bath (i.e. at the surface of the membrane). The crosslinking agents probably diffused out of the membranes together with chloroform during the chloroform/ethanol exchange before complete solidification of the membranes. As a result, the remaining amount of available crosslinking agents was too low to achieve proper crosslinking of the membranes.

Other approaches to improve crosslinking of PEO-containing membranes, such as increasing the amount of crosslinking agents in the polymer dope, adding crosslinking agents to the coagulation bath, decreasing the volume of the coagulation bath and using toluene instead of ethanol as non-solvent were not successful (data not shown).

\subsection{PTMC membranes with PVP}

During initial fabrication of PTMC membranes with PVP, yet another strategy to photo-crosslink the membranes by introducing the crosslinking agents after LIPS before UV-treatment proved to be successful. Therefore, all PVP-containing membranes in this study were prepared in this way (see Section 2.3).

\subsubsection{PVP as a pore former}

Figure 2 shows PTMC membranes made from polymer dopes that contained PVP. Different amounts of PVP were used to tailor the porosity and pore size of the membranes. Membranes with $0.3 \mathrm{wt} \%$ PVP contained no visible pores on the air side, and their cross-section showed only a few pores. In contrast, membranes with 1, 2 or $3 \mathrm{wt} \%$ PVP had pores on both surfaces and across the entire cross-section. Pores were also visible after casting the polymer dopes with $6 \mathrm{wt} \%$ PVP. However, this did not result in proper membranes but rather in fibrous polymer meshes. Besides, the 


\section{- Chapter 4 -}

Porous PTMC membranes prepared by LIPS and photo-crosslinking

polymer dopes with 3 and $6 \mathrm{wt} \%$ PVP were less homogeneous than the others, resulting in heterogeneous membranes.

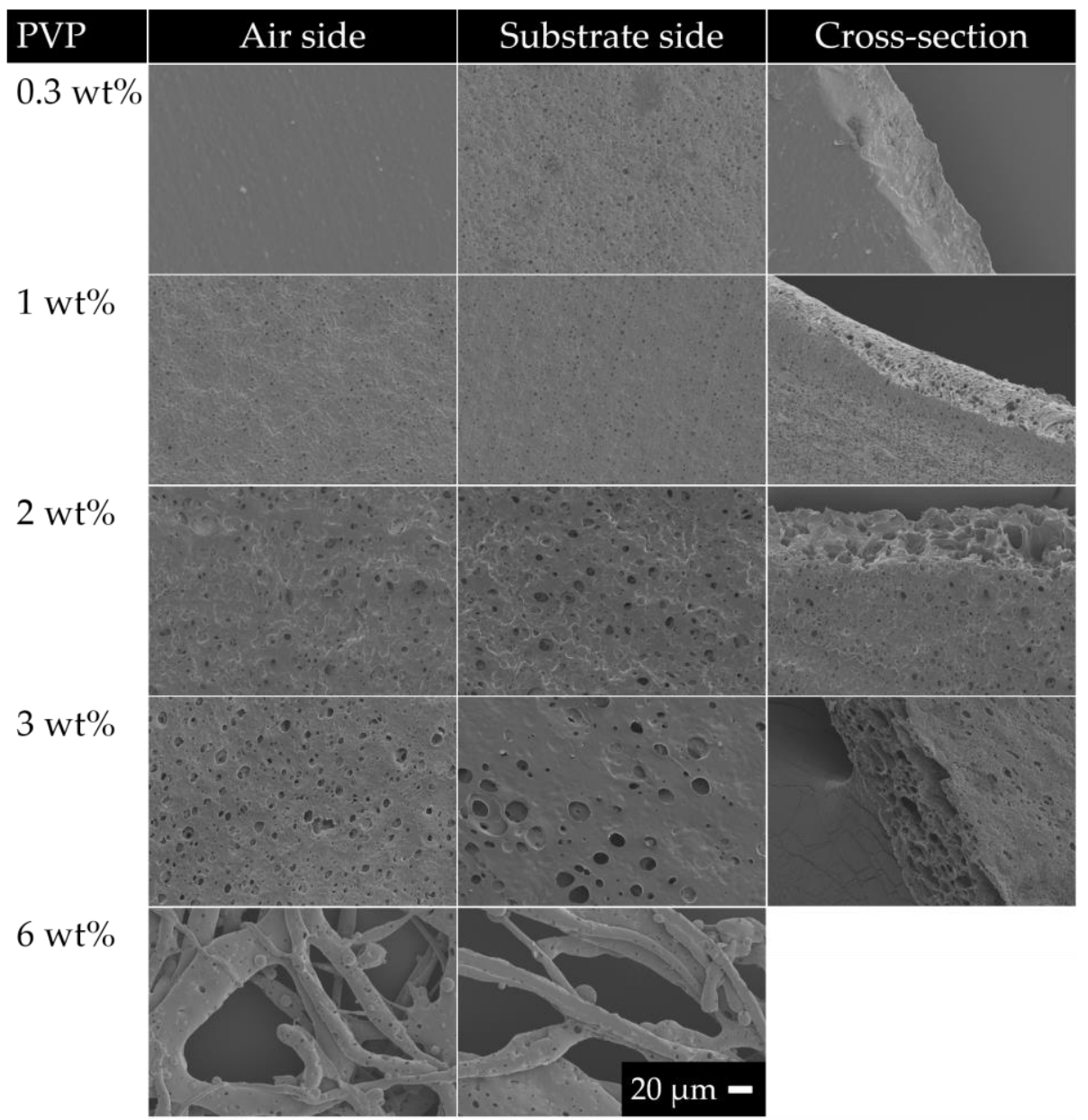

Figure 2. SEM images of crosslinked membranes made from PTMC with different concentrations of PVP as an additive. Magnification: 500x. Scale bar applies to all images. Images are representative of observations of multiple samples $(\mathrm{N}=2)$.

In general, the membrane porosity and pore size increased with increasing amount of PVP. The membranes prepared with $1 \mathrm{wt} \% \mathrm{PVP}$ had a relatively consistent porosity and pore diameter (i.e. approximately 1-3 $\mu \mathrm{m})$ across the samples. Membranes with $2 \mathrm{wt} \%$ and $3 \mathrm{wt} \%$ PVP had an increasing variation in porosity and pore diameter (i.e. approximately 1 - 
$10 \mu \mathrm{m}$ and $1-15 \mu \mathrm{m}$, respectively) (Figure 2). Thus, in terms of pore diameter, the membranes with $3 \mathrm{wt} \%$ PVP were less suitable for cell culturing. Membranes also became less homogeneous with increasing membrane size. Membranes with PVP adhered stronger to the glass plate than the membranes with PEO. This probably affected LIPS as chloroform/ethanol exchange might not occur similarly across the entire membrane, leading to heterogeneity.

Membranes with PVP shrunk during LIPS. Similar to the membranes containing PEO, the shrinkage decreased with increasing PVP amounts. However, membranes with PVP were substantially easier to handle than those with PEO. The former were less likely to fold and less sticky. Therefore, we chose to continue this study with PVP-containing membranes.

\subsubsection{Crosslinking of the membranes}

Figure 3 shows the gel content of PTMC membranes with 1, 2 or $3 \mathrm{wt} \%$ PVP, i.e. $54 \% \pm 14 \%, 69 \% \pm 15 \%$ and $87 \% \pm 5 \%$, respectively. The crosslinking was probably highly dependent on the porosity and pore size of the membranes, as the more porous membranes provided more access and surface area for the crosslinking agents to distribute better and more homogeneously through the membrane. This probably explains the lower variation in gel content of membranes with $3 \mathrm{wt} \%$ PVP compared to the others, despite the higher homogeneity of the latter membranes. Based on these data, the membranes with $2 \mathrm{wt} \%$ PVP were the most promising since they had a relatively high gel content and good membrane morphology in terms of porosity and pore size. Thus, additional experiments were done with these membranes to investigate some requirements needed for cell culturing. 


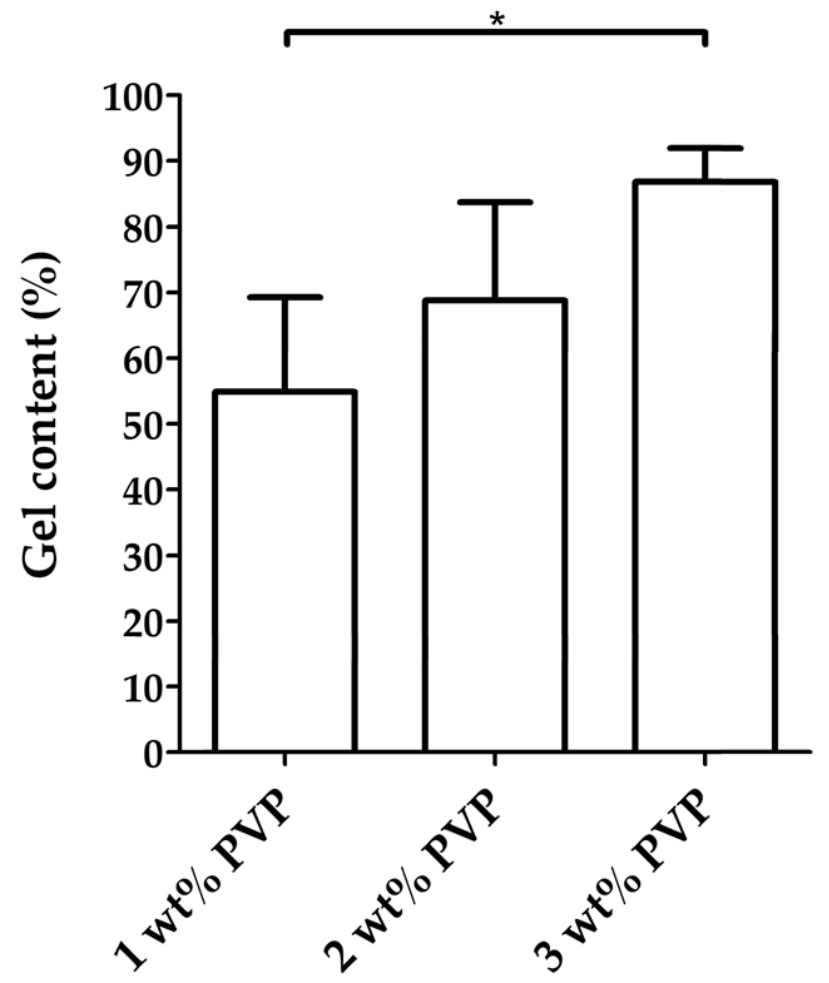

Figure 3. Gel content of PTMC membranes prepared with different concentrations of PVP. The membranes were incubated with crosslinking agents after LIPS and subsequently photo-crosslinked. The capped line with asterisk indicates a significant difference $(P<0.05)$. Depicted are averages $\pm S D(N \geq 3)$.

\subsubsection{Stability of membrane morphology}

Figure 4 shows the membrane morphology of PTMC membranes with 2 wt $\%$ PVP that were submerged in cell culture medium for two weeks and respective membranes which were not kept in culture medium. Moreover, two different membrane types were used. One membrane was photo-crosslinked (i.e. Figure 4 , left column, $82 \%$ gel content), and the other one was not (i.e. Figure 4 , right column, $0 \%$ gel content). Both membranes were porous before submersion in culture medium. However, the non-crosslinked membrane showed a completely dense morphology after two weeks in the culture medium, while the morphology of the crosslinked membrane was unchanged. The loss of 
Porous PTMC membranes prepared by LIPS and photo-crosslinking

porosity in the non-crosslinked membrane was similar to observations of LIPS-based PTMC membranes from Papenburg et al. [21].

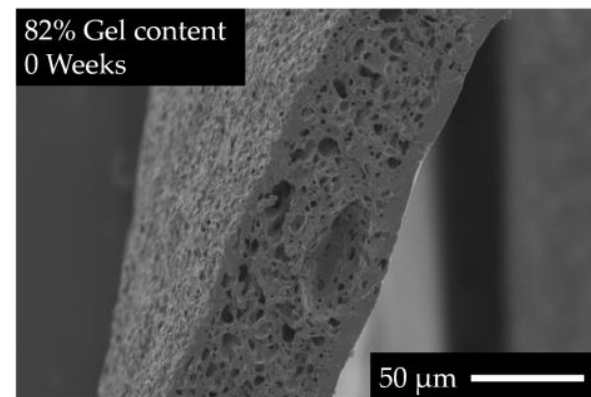

\section{$82 \%$ Gel content}

2 Weeks

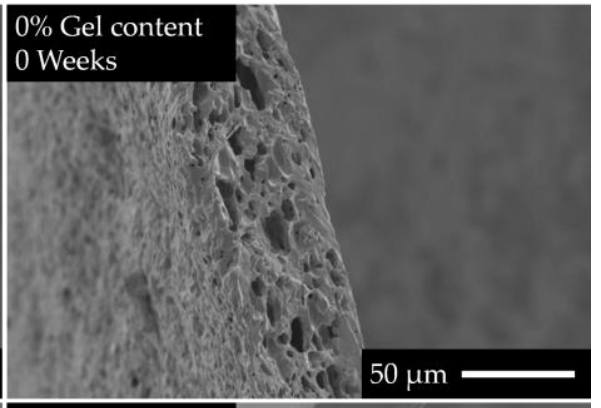

$0 \%$ Gel content

2 Weeks

$50 \mu \mathrm{m}$

$50 \mu \mathrm{m}$

Figure 4. SEM images of PTMC membranes prepared with $2 \mathrm{wt} \%$ PVP which were crosslinked (left: $82 \%$ gel content) or not crosslinked (right: $0 \%$ gel content). Morphology of membranes was assessed before and after two weeks of incubation in complete cell culture medium. Shown are the cross-sections of the membranes. Magnification: 500x. Scale bar applies to all images. Images are representative of observations of multiple samples $(\mathrm{N}=2)$.

\subsubsection{Clean water transport across the membranes}

Figure 5 shows the water flux of the crosslinked PTMC membranes prepared with $2 \mathrm{wt} \%$ PVP. The flux increased linearly with increasing TMP, as expected suggesting that samples did not compact and that the polymer network in the membranes had good integrity, confirming the results of the incubation in cell culture medium (Figure 4). The water permeance of the membranes was approximately 2,100 $\pm 1,560$ $\mathrm{L} /\left(\mathrm{m}^{2} \cdot \mathrm{h} \cdot \mathrm{bar}\right)$, in the same range as that of commercial PET membranes, but lower than that of commercial PC membranes (i.e. 3,000 $\pm 130 \mathrm{~L} /\left(\mathrm{m}^{2} \cdot \mathrm{h} \cdot \mathrm{bar}\right)$ and $25,000 \pm 4,200 \mathrm{~L} /\left(\mathrm{m}^{2} \cdot \mathrm{h} \cdot \mathrm{bar}\right)$, respectively) [30]. These data indicate 
good interconnectivity of the pores. However, there was a high variation between samples (Figure 5), which is probably caused by the heterogeneity of the membranes as discussed previously (see Section 3.2.1).

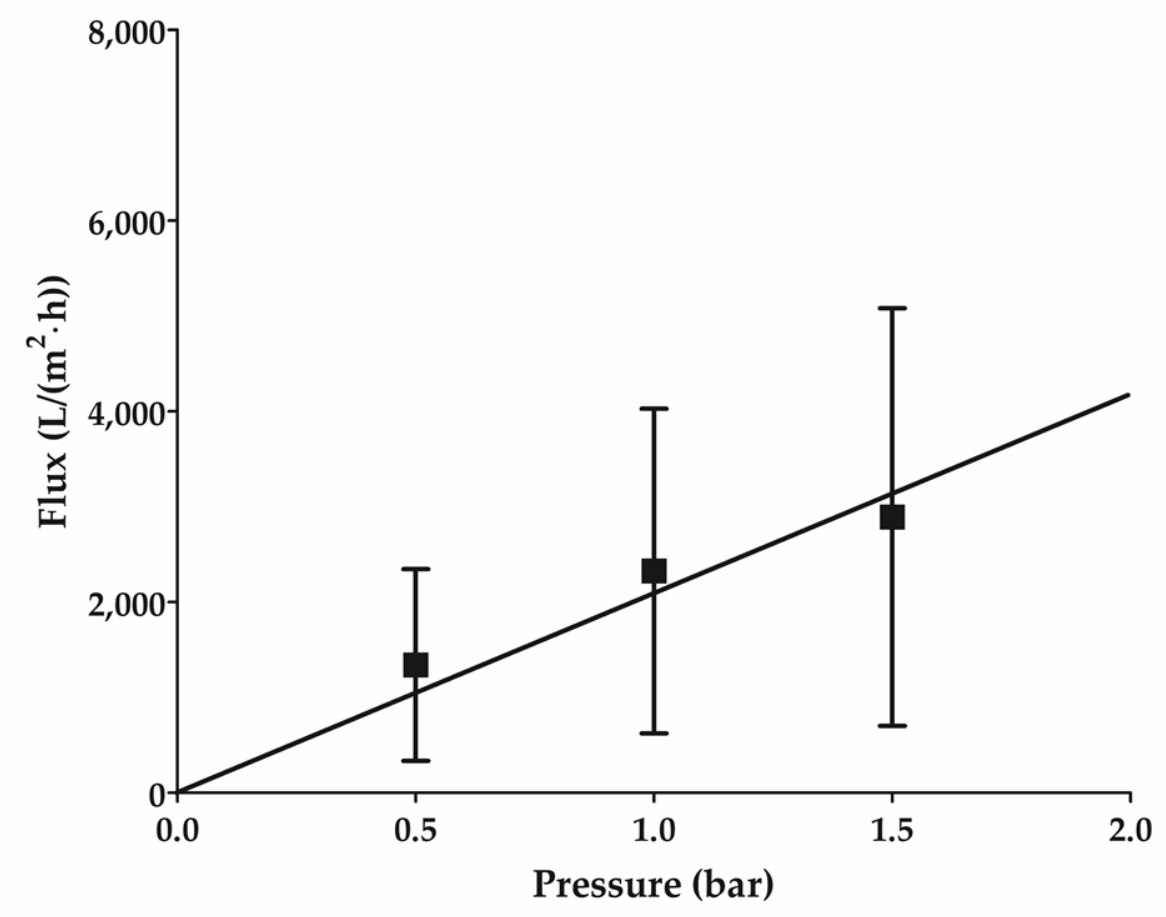

Figure 5. Water flux of crosslinked PTMC membranes prepared with $2 \mathrm{wt} \% \mathrm{PVP}$, measured after pre-compaction at different TMP. Shown are average fluxes $\pm \mathrm{SD}$ $(\mathrm{N}=5)$. The line represents the best linear fit.

\section{Conclusions}

This study showed that LIPS is a promising technique to fabricate porous membranes from PTMC. However, optimal membrane crosslinking is required but difficult to achieve stable membranes. We combined LIPS and photo-crosslinking to fabricate form-stable and porous PTMC membranes, by incubating the membranes with crosslinking agents between LIPS and UV-treatment. Furthermore, by varying the pore former, i.e. PEO and PVP, as well as its molecular weight and 
concentration in the polymer dope, membrane properties such as pore size, porosity and ease of handling were tailored.

Application of the above-mentioned crosslinking method in combination with a 3:2 weight ratio of PTMC to PVP, produced form-stable membranes with pore diameters ranging from 1 to $10 \mu \mathrm{m}$, which are expected to be suitable for cell culturing. However, the fabrication method requires further optimisation to yield reproducible membranes in terms of morphology and water flux. Nonetheless, the combination of photo-crosslinking and LIPS is novel and could be suitable for preparation of porous membranes using other polymers.

\section{References}

1. Chapter 2 of this thesis

2. Lee, J.H.; Lee, S.J.; Khang, G.; Lee, H.B. Interaction of fibroblasts on polycarbonate membrane surfaces with different micropore sizes and hydrophilicity. J Biomater. Sci. Polym. Ed. 1999, 10, 283-294. DOI: 10.1163/156856299X00351.

3. Gotoh, K.; Yasukawa, A.; Taniguchi, K. Water contact angles on poly(ethylene terephthalate) film exposed to atmospheric pressure plasma. J. Adhes. Sci. Technol. 2011, 25, 307-322. DOI: 10.1163/016942410X511114.

4. Lai, Y.S.; Chen, W.C.; Huang, C.H.; Cheng, C.K.; Chan, K.K.; Chang, T.K. The effect of graft strength on knee laxity and graft in-situ forces after posterior cruciate ligament reconstruction. PLoS One. 2015, 10(5). DOI: 10.1371/journal.pone.0127293.

5. Bat, E.; Zhang, Z.; Feijen, J.; Grijpma, D.W.; Poot, A.A. Biodegradable elastomers for biomedical applications and regenerative medicine. Regen. Med. 2014, 9(3), 385-398. DOI: 10.2217/RME.14.4.

6. Terzini, M.; Bignardi, C.; Castagnoli, C.; Cambieri, I.; Zanetti, E.M.; Audenino, A.L. Ex Vivo Dermis Mechanical Behavior in Relation to Decellularization Treatment Length. Open Biomed. Eng. J. 2016, 10(1), 34-42. DOI: 10.2174/1874120701610010034.

7. Butcher, D.T.; Alliston, T.; Weaver, V.M. A tense situation: forcing tumour progression. Nat. Rev. Cancer. 2009, 9(2), 108-122. DOI: 10.1038/nrc2544.

8. Huh, D.; Kim, H.J.; Fraser, J.P.; Shea, D.E.; Khan, M.; Bahinski, A.; Hamilton, G.A.; Ingber, D.E. Microfabrication of human organs-on-chips. Nat. Protoc. 2013, 8(11), 2135-2157. DOI: 10.1038/nprot.2013.137.

9. Huh, D.; Hamilton, G.A.; Ingber, D.E. From 3D cell culture to organs-on-chips. Trends Cell Biol. 2011, 21(12), 745-754. DOI: 10.1016/j.tcb.2011.09.005.

10. Huh, D.; Leslie, D.C.; Matthews, B.D.; Fraser, J.P.; Jurek, S.; Hamilton, G.A.; Thorneloe, K.S.; McAlexander, M.A.; Ingber, D.E. A human disease model of 


\section{- Chapter 4 -}

Porous PTMC membranes prepared by LIPS and photo-crosslinking

drug toxicity-induced pulmonary edema in a lung-on-a-chip microdevice. Sci. Transl. Med. 2012, 4(159). DOI: 10.1126/scitranslmed.3004249.

11. Huh, D.; Torisawa, Y.S.; Hamilton, G.A.; Kim, H.J.; Ingber, D.E. Microengineered physiological biomimicry: organs-on-chips. Lab Chip. 2012, 12(12), 2156-2164. DOI: 10.1039/c2lc40089h.

12. Chen, Y.F.; Chan, H.N.; Michael, S.A.; Shen, Y.S.; Chen, Y.; Tian, Q.; Huang, L.; $\mathrm{Wu}, \mathrm{H}$. A microfluidic circulatory system integrated with capillary-assisted pressure sensors. Lab Chip. 2017, 17(4), 653-662. DOI: 10.1039/c6lc01427e.

13. Di Caprio, G.; Stokes, C.; Higgins, J.M.; Schonbrun, E. Single-cell measurement of red blood cell oxygen affinity. Proc. Natl. Acad. Sci. USA. 2015, 112(32), 99849989. DOI: 10.1073/pnas.1509252112.

14. Ochsner, M.; Dusseiller, M.R.; Grandin, H.M.; Luna-Morris, S.; Textor, M.; Vogel, V.; Smith, M.L. Micro-well arrays for 3D shape control and high resolution analysis of single cells. Lab Chip. 2007, 7(8), 1074-1077. DOI: 10.1039/b704449f.

15. Toepke, M.W.; Beebe, D.J. PDMS absorption of small molecules and consequences in microfluidic applications. Lab chip. 2006, 6(12), 1484-1486. DOI: 10.1039/b612140c.

16. Van Meer, B.J.; de Vries, H.; Firth, K.; van Weerd, J.; Tertoolen, L.; Karperien, H.; Jonkheijm, P.; Denning, C.; IJzerman, A.P.; Mummery, C.L. Small molecule absorption by PDMS in the context of drug response bioassays. Biochem. Bioph. Res. Co. 2017, 482(2), 323-328. DOI: 10.1016/j.bbrc.2016.11.062

17. Wala, J.; Maji, D.; Das, S. Influence of physico-mechanical properties of elastomeric material for different cell growth. Biomed. Mater. 2017, 12(6). DOI: 10.1088/1748-605X/aa7e81.

18. Bat, E.; Feijen, J.; Grijpma, D.W. Biodegradable elastomeric networks: highly efficient cross-linking of poly(trimethylene carbonate) by gamma irradiation in the presence of pentaerythritol triacrylate. Biomacromolecules. 2010, 11(10), 2692-2699. DOI: 10.1021/bm1007234.

19. Pêgo, A.P. Preparation and in vivo performance of degradable two-ply nerve guides based on (co)polymers of trimethylene carbonate and $\varepsilon$-caprolactone. Ph.D. thesis, University of Twente, Enschede, The Netherlands, November 2002.

20. Pêgo, A.P.; Siebum, B.; Van Luyn, M.J.; Gallego y Van Seijen, X.J.; Poot, A.A.; Grijpma, D.W.; Feijen, J. Preparation of degradable porous structures based on 1,3-trimethylene carbonate and D,L-lactide (co)polymers for heart tissue engineering. Tissue Eng. 2003, 9(5), 981-994. DOI: 10.1089/107632703322495628.

21. Papenburg, B.J.; Schuller-Ravoo, S.; Bolhuis-Versteeg, L.A.M.; Hartsuiker, L.; Grijpma, D.W.; Feijen, J.; Wessling, M.; Stamatialis, D. Designing porosity and topography of poly(1,3-trimethylene carbonate) scaffolds. Acta. Biomater. 2009, 5(9), 3281-3294. DOI: 10.1016/j.actbio.2009.05.017.

22. Bat, E.; Kothman, B.H.M.; Higuera, G.A.; van Blitterswijk, C.A.; Feijen, J.; Grijpma, D.W. Ultraviolet light crosslinking of poly(trimethylene carbonate) for elastomeric tissue engineering scaffolds. Biomaterials. 2010, 31(33), 8696-8705. DOI: 10.1016/j.biomaterials.2010.07.102.

23. Allijn, I.; Ribeiro, M.; Poot, A.A.; Passier, R.; Stamatialis, D. Membranes for Modelling Cardiac Tissue Stiffness In Vitro Based on Poly(trimethylene 


\section{- Chapter 4 -}

Porous PTMC membranes prepared by LIPS and photo-crosslinking

carbonate) and Poly(ethylene glycol) Polymers. Membranes. 2020, 10(10), E274. DOI: 10.3390/membranes10100274.

24. Chapter 3 of this thesis.

25. Pêgo, A.P.; Poot, A.A.; Grijpma, D.W.; Feijen, J. Copolymers of trimethylene carbonate and epsilon-caprolactone for porous nerve guides: synthesis and properties. Biomater. Sci. Polym. Ed. 2001, 12(1), 35-53. DOI: 10.1163/156856201744434.

26. Pêgo, A.P.; Grijpma, D.W.; Feijen, J. Enhanced mechanical properties of 1,3trimethylene carbonate polymers and networks. Polymer. 2003, 44(21), 6495-6504. DOI: 10.1016/S0032-3861(03)00668-2.

27. Astakhov, E.; Kolganov, I.; Klinshpont, E.; Tsarin, P.; Kalacheva, A. Influence of polyvinylpyrrolidone on morphology, hydrophilicity, and performance of polyethersulfone microfiltration membranes. Petroleum Chemistry. 2013, 52. DOI: 10.1134/S0965544112080026.

28. Bat, E.; Kothman, B.H.M.; Higuera, G.A.; van Blitterswijk, C.A.; Feijen, J.; Grijpma, D.W. Ultraviolet light crosslinking of poly(trimethylene carbonate) for elastomeric tissue engineering scaffolds. Biomaterials. 2010, 31(33), 8696-8705. DOI: 10.1016/j.biomaterials.2010.07.102.

29. Bat, E.; van Kooten, T.G.; Feijen, J.; Grijpma, D.W. Resorbable elastomeric networks prepared by photocrosslinking of high-molecular-weight poly(trimethylene carbonate) with photoinitiators and poly(trimethylene carbonate) macromers as crosslinking aids. Acta. Biomater. 2011, 7(5), 1939-1948. DOI: 10.1016/j.actbio.2011.01.010.

30. Chapter 5 of this thesis. 
- Chapter 4 -

Porous PTMC membranes prepared by LIPS and photo-crosslinking 


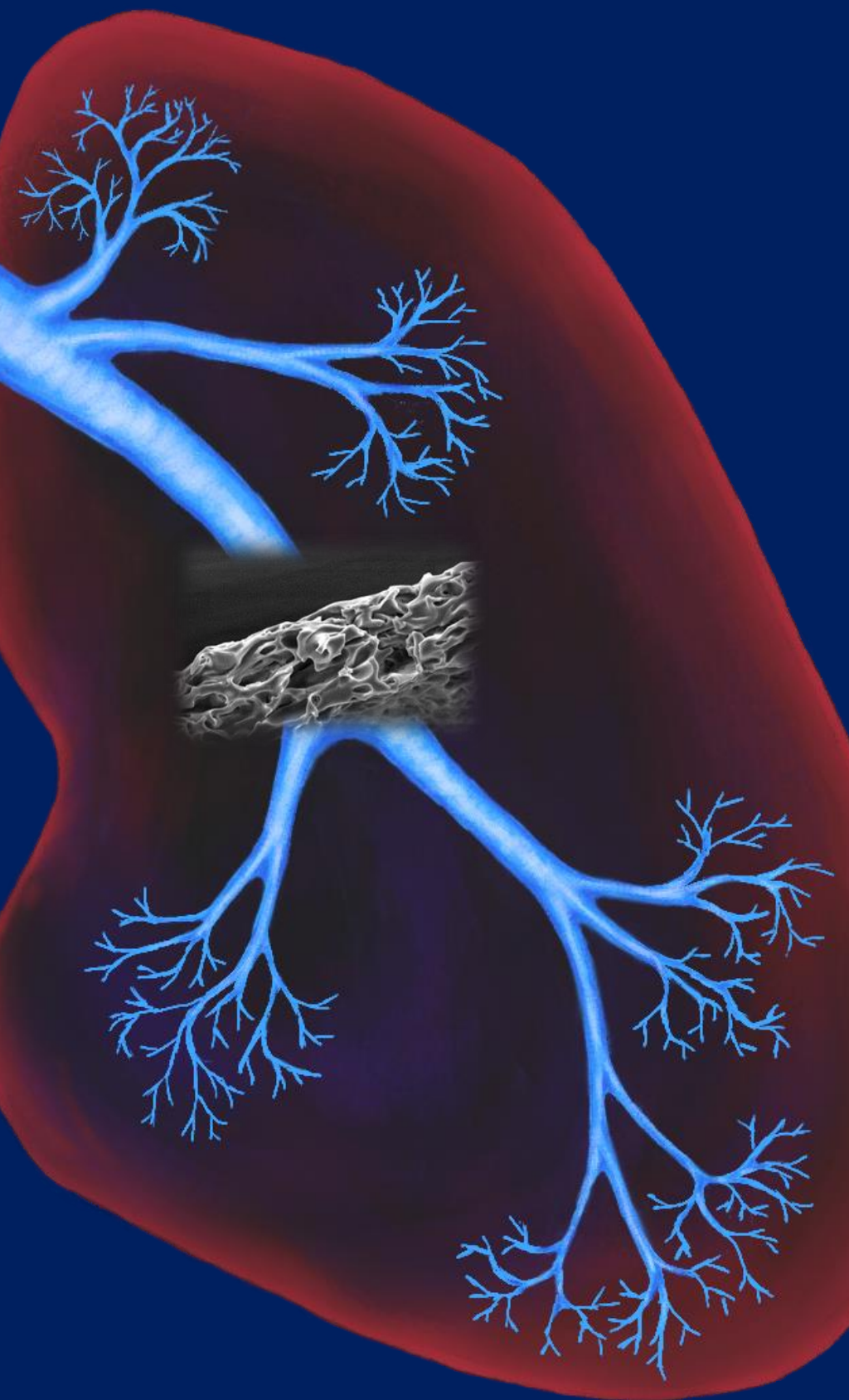




\section{- Chapter 5 -}

\section{Development of porous and flexible PTMC membranes for in vitro organ models fabricated by evaporation-induced phase separation}

Thijs Pasman 1, Danielle Baptista 2, Sander van Riet 3, Roman, K. Truckenmüller 2, Pieter S. Hiemstra ${ }^{3}$, Robbert J. Rottier ${ }^{4}$, Dimitrios Stamatialis ${ }^{1}$, André A. Poot ${ }^{1}$

1 Technical Medical (TechMed) Centre, Department of Biomaterials Science and Technology, Faculty of Science and Technology, University of Twente, 7522 NB Enschede, The Netherlands

2 Department of Instructive Biomaterials Engineering, MERLN Institute for Technology-Inspired Regenerative Medicine, Maastricht University, 6229 ER Maastricht, The Netherlands

3 Department of Pulmonology, Leiden University Medical Centre, 2300 RC Leiden, The Netherlands

4 Department of Pediatric Surgery and Cell Biology, Erasmus MC-Sophia Children's Hospital, 3000 CB Rotterdam, The Netherlands

This chapter was adapted from: Pasman, T.; Baptista, D.; van Riet, S.; Truckenmüller, R.K.; Hiemstra, P.S.; Rottier, R.J.; Stamatialis, D.; Poot, A.A. Development of porous and flexible PTMC membranes for in vitro organ models fabricated by evaporation-induced phase separation. Membranes. 2020, 10(11), 330. DOI: 10.3390/membranes10110330. 


\section{Abstract}

Polymeric membranes are widely applied in biomedical applications, including in vitro organ models. In such models, they are mostly used as supports on which cells are cultured to create functional tissue units of the desired organ. To this end, the membrane properties, e.g. morphology and porosity, should match the tissue properties. Organ models of dynamic (barrier) tissues, e.g. lung, require flexible, elastic and porous membranes. Thus, membranes based on poly(dimethyl siloxane) (PDMS) are often applied, which are flexible and elastic. However, PDMS has low cell adhesive properties and displays small molecule ad- and absorption. Furthermore, the introduction of porosity in these membranes requires elaborate methods. In this work, we aim to develop porous membranes for organ models based on poly(trimethylene carbonate) (PTMC): a flexible polymer with good cell adhesive properties which has been used for tissue engineering scaffolds, but not in in vitro organ models. For developing these membranes, we applied evaporation-induced phase separation (EIPS), a new method in this field based on solvent evaporation initiating phase separation, followed by membrane photocrosslinking. We optimised various processing variables for obtaining form-stable PTMC membranes with average pore sizes between 5 to $8 \mu \mathrm{m}$ and water permeance in the microfiltration range $(17,000-41,000$ $\mathrm{L} /\left(\mathrm{m}^{2} \cdot \mathrm{h} \cdot \mathrm{bar}\right)$. Importantly, the membranes are flexible and are suitable for implementation in in vitro organ models.

Keywords: membranes; poly(trimethylene carbonate) (PTMC); evaporation-induced phase separation (EIPS); photo-crosslinking; in vitro organ models

\section{Introduction}

With increasing costs to develop new medicines and treatments, and ethical and scientific concerns related to the use of animal models, there is an increasing need for developing in vitro organ-mimicking models [1]. These models should generate more reliable results than traditional models, such as those based on cell cultures in dishes on tissue culture 
plastic and those in Transwell@ inserts under static conditions, since they would consist of functional tissue units that are actively perfused, which better represent the native tissue.

Polymeric membranes are a vital part of many organ models [1-10]. They provide a surface for the cells to attach and grow and often (partly) substitute the extracellular matrix (e.g. the basal membrane). They often require specific coating for the growth of (primary) cells. Besides, the membrane properties should promote physiological cell behaviour, and thus match the properties of the desired tissue or organ. Importantly, the membranes need to be porous to allow communication between the cells of co-culture models and enable nutrient, metabolite and gas transport to and from the cells. Moreover, the membranes used in organ models of dynamic tissues, such as the lung and heart, need to match the mechanical properties of these organs since these properties affect cellular behaviour [1].

Many of the current in vitro systems include commercial membranes based on poly(ethylene terephthalate) (PET) or poly(bisphenol-Acarbonate) (PC) [1]. Most of these membranes are made porous by ion track etching, which provides great and independent control over pore size and density, and enables a wide range of membrane pore sizes (i.e. $0.4-8 \mu \mathrm{m})$ [1]. PET and PC are transparent, which is beneficial for cell imaging techniques. However, both have relatively high elastic moduli (E-moduli), of 2-3 GPa [11] and 2-2.4 GPa [12], respectively. These moduli are quite appropriate for matching those of cortical bone (6-34 GPa) [1317], cancellous bone (0.1 to $2 \mathrm{GPa})[15,16]$, and specific ligaments $(366$ $\mathrm{MPa}$ [ [16], but far exceed the moduli of most tissues which are in the range of several $\mathrm{kPa}$ to $\mathrm{MPa}$ [16-19].

Other culture systems implement membranes based on poly(dimethyl siloxane) (PDMS) $[1-4,6,20,21]$. The E-modulus of PDMS is easily tunable from $4 \mathrm{kPa}$ to several $\mathrm{MPa}$ [22], which facilitates cyclic stretch of the membranes and cells $[2,4,6]$. Moreover, PDMS is transparent and easy to 
prepare [23]. However, the fabrication of porous PDMS membranes suitable for cell culturing is difficult [24,25], requiring rather elaborate methods (i.e. mostly soft lithography) [1]. Furthermore, their pore density is often low [2,10], which potentially prevents optimal cell communication and nutrient delivery to cells. Besides, cell attachment on PDMS is low [26]. Therefore, PDMS needs additional coatings, surface modifications, or both, to accommodate cell growth [26]. Finally, PDMS is known for its ad- and absorption of small molecules, potentially affecting outcomes of (drug) studies [27,28].

In this work, we describe the development of membranes for organ-onchip (OOC) models based on the polymer poly(trimethylene carbonate) (PTMC), which is transparent [23], non-cytotoxic [29,30] and has good cell adhesive properties [30-32]. PTMC adsorbs cell culture medium components [31], which is beneficial for cell adhesion but it can also be coated [33-35] for improving cell adhesion. This could be useful for (primary) cells that require specific coatings. Earlier studies have also shown that PTMC-based structures (e.g. scaffolds and films) have good mechanical properties, such as high elongation at break, good toughness, relatively low E-moduli compared to PC and PET and low permanent deformation [29,32,33]. Moreover, the mechanical properties of crosslinked PTMC networks can be tuned to match those of the targeted tissue by changing the molecular weight of the PTMC [36] and the crosslinking conditions [29,32]. In addition, their mechanical properties are good and remain unchanged when wetted $[33,37,38]$ due to a low water uptake $($ i.e. $<2 \%)[32,37,39]$. Furthermore, it is biodegradable via surface erosion, while retaining its properties until degradation is almost complete [32,39-41], which is beneficial for in vivo applications [34,41,42]. Furthermore, its degradation does not produce any toxic products $[29,40]$. All these characteristics have made it an interesting and successful material for scaffolds, films and porous membranes for biomedical applications, both in vitro [30-32] and in vivo [34,41,42]. Nevertheless, PTMC is amorphous and has a low glass transition temperature $\left(\mathrm{T}_{\mathrm{g}}\right)$ of $-17^{\circ} \mathrm{C}[31,40]$. Therefore, crosslinking of the PTMC is needed to preserve 
its porous structure. Earlier studies implemented temperature-induced phase separation (TIPS) [43] for the fabrication of porous PTMC membranes and liquid-induced phase separation (LIPS) [31] for porous PTMC scaffolds. However, the fabrication of membranes by TIPS was not reproducible [43]. LIPS resulted in reproducible PTMC scaffolds for cell culture, which could be crosslinked by gamma-irradiation. Nevertheless, the process duration of crosslinking was rather long leading to a substantial loss of membrane porosity [31]. Photo-crosslinking of PTMC scaffolds is possible by using crosslinking agents [32,39]. However, crosslinking the LIPS-based PTMC scaffolds would not be straightforward due to the large volumes of non-solvents used with LIPS, which could lead to solvation and loss of polymer dope components (i.e. the crosslinking agents).

In this study, we aim to overcome the above limitations by preparing crosslinked PTMC membranes via evaporation-induced phase separation (EIPS) combined with photo-crosslinking (Scheme 1). For EIPS, a polymer dope which contains a volatile solvent and a non-solvent for the polymer is cast on a suitable surface. Evaporation of the volatile solvent increases the concentration of polymer and non-solvent to a point where phase separation occurs. The non-solvent, which is non-volatile or significantly less volatile than the solvent, coalesces into droplets inside the polymer layer and is present long enough for solidification of the polymer. In this study, polymer dopes containing PTMC and poly(ethylene oxide) (PEO), the latter as pore former and pore stabiliser [31,37], chloroform (solvent) and a non-solvent (i.e. ethanol, propanol, butanol or hexanol), are cast on silicon wafers. Immediately after EIPS, the membranes can be photocrosslinked with UV-light to preserve the porosity of the amorphous PTMC structures. To enable photo-crosslinking, the polymer dope also contains pentaerythritol triacrylate (PETA) and Irgacure 2959 as crosslinking agents, as they have been used to successfully photocrosslink porous and cell-adhesive PTMC-based scaffolds [32,39]. After crosslinking, the solidified polymer layer is washed to remove the nonsolvent, which reveals the pores created by the non-solvent. Volumes of 
non-solvent used in EIPS are much smaller than the non-solvent bath used in LIPS. Thus, we anticipate that, with EIPS, it is less likely that components such as the crosslinking agents dissolve into the non-solvent.

Here, we tailored the PTMC membrane morphology and porosity by a detailed investigation of various EIPS parameters, including the environmental conditions (e.g. humidity and temperature), the type of non-solvent (a series of alcohols) and the polymer dope composition (the molecular weight (MW) of PTMC and the amount of non-solvent). The developed membranes were characterised in detail concerning the network chemistry, their mechanical properties, morphology (i.e. roughness, porosity, pore size) and transport properties (water transport). Besides, they were compared to membranes used in commercial inserts (PET and PC) and to PDMS membranes reported in literature.

\section{Materials and methods}

\subsection{Materials}

Trimethylene carbonate (TMC, Boehringer Ingelheim, Ingelheim, Germany), stannous octoate ( $\mathrm{Sn}(\mathrm{Oct}) 2$, Spectrum Chemical MFG Corp., New Brunswick, NJ, USA), chloroform (Merck Millipore, Darmstadt, Germany, Emparta cat. 1.07024.2500, analytical grade), ethanol for membrane washing (Boom B.V., Meppel, The Netherlands, cat. 84050065.5000), ethanol for PTMC precipitation and as non-solvent (Merck Millipore, Darmstadt, Germany, Emsure cat. 1.00983.1000, analytical grade), propan-1-ol (Sigma-Aldrich, Darmstadt, Germany, cat. 279544), butan-1-ol (Sigma-Aldrich, Darmstadt, Germany, cat. 281549), hexan-1-ol (Sigma-Aldrich, Darmstadt, Germany, cat. 471402), poly(ethylene oxide) (PEO) 5,000,000 g/mol (Sigma-Aldrich, Darmstadt, Germany, cat. 189472), pentaerythritol triacrylate (PETA) (Sigma-Aldrich, Darmstadt, Germany, cat. 246794), 2-Hydroxy-4'-(2-hydroxyethoxy)-2methylpropiophenone (Irgacure 2959) (Sigma-Aldrich, Darmstadt, Germany, cat. 410896) and deuterated chloroform (Sigma-Aldrich, Darmstadt, Germany, cat. 151823) were all used as received. Ultrapure 
water (MilliQ water) was produced by a Millipore Advantage A10 (Merck Millipore, Darmstadt, Germany). Additional information about the function and MW of the components used in the polymer dopes can be found in Table 1.

\subsection{Poly(trimethylene carbonate) (PTMC) synthesis}

Linear PTMC was synthesised by ring-opening polymerisation of TMC for three days at $130{ }^{\circ} \mathrm{C}$ under vacuum in a silanised, dry glass ampoule. MilliQ water (weight ratio of TMC to water of 180:1 and 90:1) and Sn(Oct) (weight ratio of TMC to $\mathrm{Sn}(\mathrm{Oct})_{2}$ of 90:1) were added as initiator and catalyst, respectively. Afterwards, the PTMC was dissolved in chloroform and precipitated in a ten-fold volume of cold ethanol for purification. It was then dried under vacuum at $\pm 20^{\circ} \mathrm{C}$ until constant weight.

One-point viscometry was used to determine the weight average molecular weight, i.e. the $\mathrm{Mv}$, of the PTMC. The Mv of the PTMC is referred to in the following as $\mathrm{MW}$, similar to the $\mathrm{MW}$ of the other polymers. Viscometry measurements were performed on PTMC dissolved in chloroform at $25{ }^{\circ} \mathrm{C}$ using a $0 \mathrm{C}$ Ubbelohde Viscometer $(\mathrm{N}=$ 3 ). The intrinsic viscosities were determined by calculating the relative and specific viscosities and using the Solomon-Ciuta equation. We made PTMC with two different intrinsic viscosities, i.e. $8.3 \mathrm{dL} / \mathrm{g}$ and $4.6 \mathrm{dL} / \mathrm{g}$. Using the Mark-Houwink equation (see Equation (1) below), with ' $\mathrm{K}$ ' = $2.43 \times 10^{-4}$ and ' $a$ ' $=0.74$ [40], the MW's of the two PTMC batches were estimated to be $1,300 \mathrm{~kg} / \mathrm{mol}$ and $600 \mathrm{~kg} / \mathrm{mol}$, respectively.

$$
\text { Intrinsic viscosity }[\eta]=\mathrm{K} \cdot \mathrm{MW}^{\mathrm{a}}
$$




\subsection{Membrane fabrication}

Polymer dopes with different compositions were prepared in glass jars. They always contained $3 \mathrm{wt} \%$ of PTMC in chloroform and $0.3 \mathrm{wt} \%$ PEO of 5,000 kg/mol (Table 1), according to Papenburg et al. [31]. PETA and Irgacure $2959(0.2 \mathrm{wt} \%$ and $0.01 \mathrm{wt} \%$ of the total polymer dope, respectively) were added as crosslinking aid [32] and photoinitiator [39], respectively. The remaining part of the dopes comprised of solely chloroform or a combination of a non-solvent (i.e. ethanol, propanol, butanol or hexanol) and chloroform. We made two different types of dopes:

A. For the investigation of the effect of non-solvent type on the formation of porous membranes, we used polymer dopes of PTMC of $1,300 \mathrm{~kg} / \mathrm{mol}$ and different alcohols as non-solvents. These dopes always contained $3 \mathrm{wt} \%$ non-solvent and $93.5 \mathrm{wt} \%$ chloroform.

B. For the investigation of the effect of the MW of PTMC and the nonsolvent amount on the formation of porous membranes, polymer dopes contained PTMC of either 1,300 kg/mol or $600 \mathrm{~kg} / \mathrm{mol}$. Furthermore, they contained different amounts of hexanol as a non-solvent, i.e. $0,3,6$ or $9 \mathrm{wt} \%$ of the total polymer dope. Membranes fabricated from PTMC with a MW of $600 \mathrm{~kg} / \mathrm{mol}$ and 0, 3, 6 or $9 \mathrm{wt} \%$ hexanol are in the following referred to as M0, M1, M2 and M3, respectively (Table 2). 
- Chapter 5 -

Porous and flexible PTMC membranes fabricated by EIPS

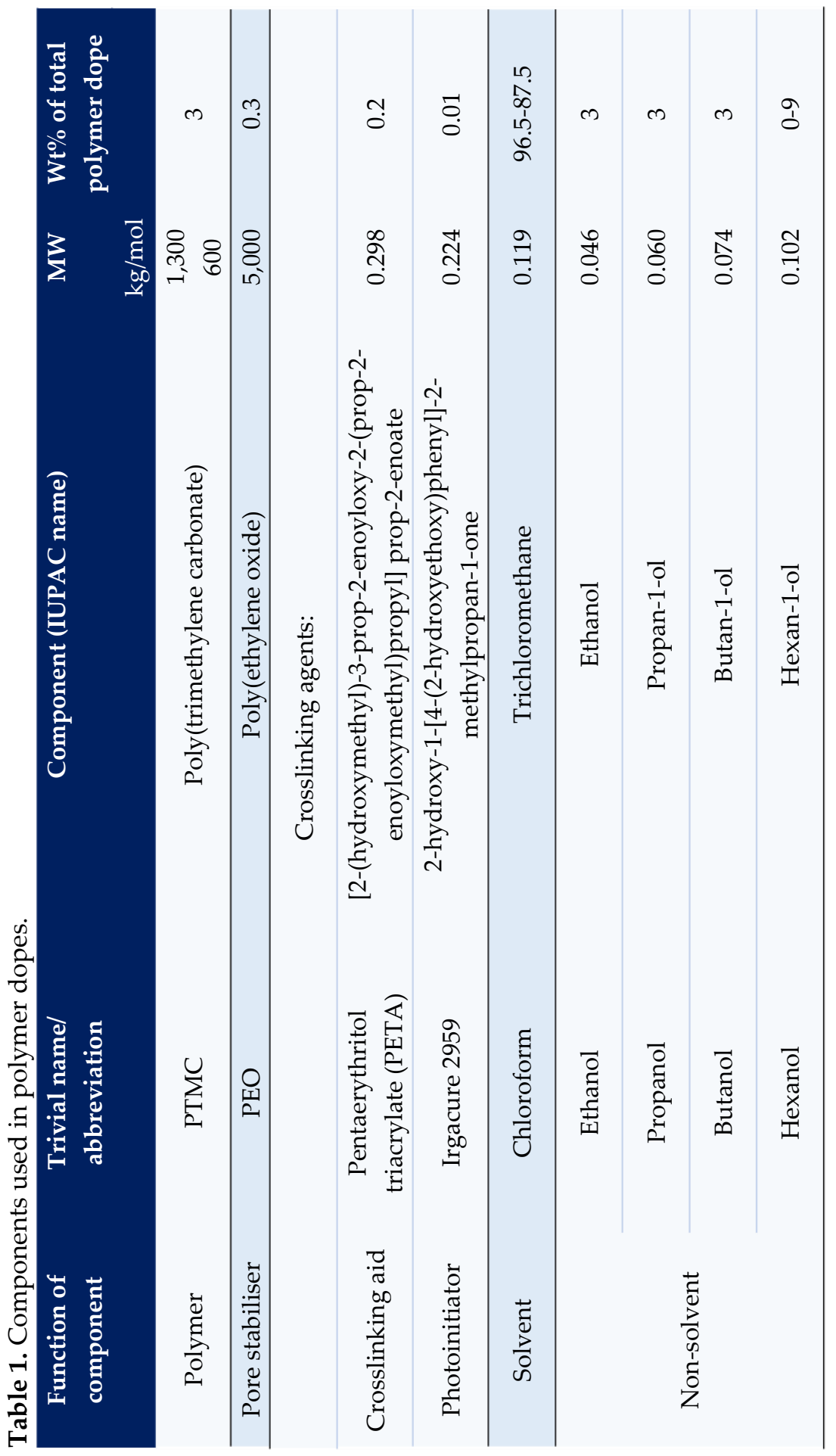


Jars containing the polymer dopes were sealed with a lid and parafilm, and the polymer dopes were stirred at an ambient temperature of 19-20 ${ }^{\circ} \mathrm{C}$ in the dark for five days (Scheme 1A). The membranes were cast by pouring the polymer solutions on silicon wafers, after which a casting knife, adjusted to a casting gap of $500 \mu \mathrm{m}$, was used to create a $500 \mu \mathrm{m}$ thick layer (Scheme 1B). Casting and fabrication of the membranes were done in a fume hood at $19-20{ }^{\circ} \mathrm{C}$ with a constant airflow of $0.75 \mathrm{~m} / \mathrm{s}$. This airflow was applied across the surface of the cast polymer layers to facilitate the removal of chloroform vapours above the membrane. The different dopes were cast in different conditions:

A. Polymer dopes with PTMC of $1,300 \mathrm{~kg} / \mathrm{mol}$ and different alcohols as non-solvents were cast at a humidity of $50 \%$. Multiple membranes were cast $(\mathrm{N}=3)$.

B. The dopes with different hexanol amounts, including those for M0-M3, were cast at $60 \%$ humidity, created by a humidifier (PureMate ${ }^{\circledR}$ PM 702, PureMate, Birmingham, UK). Moreover, the silicon wafers containing cast polymer layers of those dopes were immediately placed on a cold glass plate of $-25^{\circ} \mathrm{C}$ and $5 \mathrm{~mm}$ thick. The cold glass plate was not kept cool and thus warmed up to ambient temperature over time. Casting was performed at least five times for each condition with multiple membranes cast every time. Samples were taken from different membranes cast on different days.

The cast membranes were then left for $90 \mathrm{~min}$. in a box (Scheme 1C) in the conditions described above. During this time, EIPS occurred. The membranes and silicon wafers were then placed in a UV-box (UltraLum CEX 1500 crosslinking cabinet, Ultra Lum Enterprises, Inc., Claremont, CA, USA) for photo-crosslinking for two hours (UV-light at $254 \mathrm{~nm}$ and an intensity of $5 \mathrm{~mW} / \mathrm{cm}^{2}$, measured using an optical power meter (Newport 1916-C, Irvine, CA, USA) at a distance of $3 \mathrm{~cm}$ to the light source) (Scheme 1D). Nitrogen gas, cooled by flowing through a cold trap submerged in liquid nitrogen, was flowing into the UV-machine at a rate of $4 \mathrm{~L} / \mathrm{min}$. to prevent reaction of the crosslinking agents with oxygen. 
Afterwards, the membranes were washed (Scheme 1E) to remove the nonsolvent, uncrosslinked polymer and PETA, and residual Irgacure 2959.

Polymer dope

Stirred for 5 days in

the dark at $19-20^{\circ} \mathrm{C}$

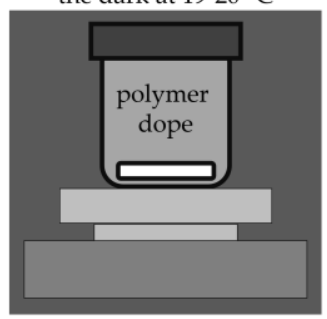

(A)

Crosslinking

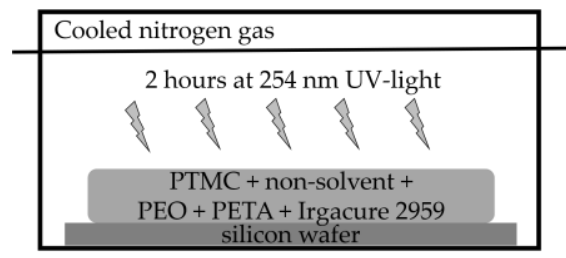

(D)

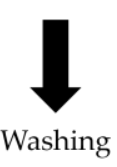

$100 \%$ ethanol, $50 \mathrm{vol} \%$ ethanol or demineralised water

non-solvent + uncrosslinked components

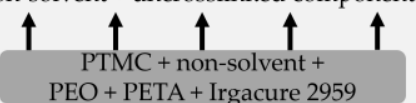

(E)
Casting

airflow $\left(19-20^{\circ} \mathrm{C}\right)$

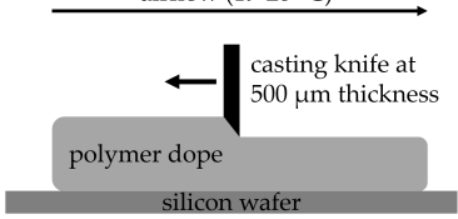

(B)

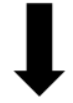

EIPS

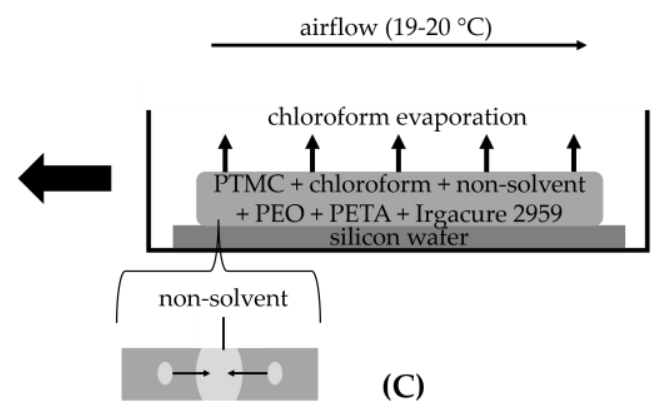

Drying
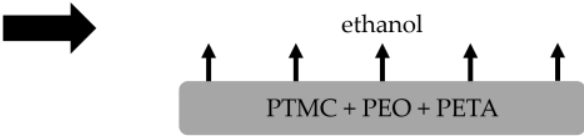

(F) 
Scheme 1. Schematic of PTMC membrane fabrication by EIPS. (A) The polymer dope is stirred for five days at $19-20^{\circ} \mathrm{C}$ in the dark. (B) The dope is then cast on silicon wafers in a $500 \mu \mathrm{m}$ thick layer using a casting knife. (C) The cast polymer layers are kept in a box for $90 \mathrm{~min}$. where chloroform evaporation and EIPS occurs. (D) The membranes are then crosslinked in a UV-box for two hours at 254 nm UV-light with cooled nitrogen gas flowing through the UV-box. (E) Membranes are then washed to remove the non-solvent and any other uncrosslinked components. (F) Finally, the membranes are rinsed with ethanol, dried and stored.

A. The membranes prepared with PTMC of $1,300 \mathrm{~kg} / \mathrm{mol}$ and different alcohols were washed in an excess of demineralised water for five days (water refreshed once every day).

B. The membranes prepared with different hexanol amounts, including $\mathrm{M} 0-\mathrm{M} 3$, were first put in an excess of $100 \%$ ethanol overnight since hexanol does not mix well with water but is miscible with ethanol. After one day, the ethanol was exchanged for $50 \mathrm{vol} \%$ ethanol in demineralised water for one day, followed by four days of demineralised water (water replaced once per day). Then, the membranes were again placed in $100 \%$ ethanol overnight to remove any residual hexanol and to exchange the water in the membranes with ethanol for better drying.

Finally, all membranes were rinsed with $100 \%$ ethanol several times and placed on Teflon paper. They were dried until constant weight at 19-20 ${ }^{\circ} \mathrm{C}$, vacuum sealed and stored at $-25^{\circ} \mathrm{C}$ (Scheme $\left.1 \mathrm{~F}\right)$. 


\subsection{The polymer network in the membranes}

\subsubsection{Gel content}

To assess whether crosslinking was successful, we performed gel content measurements. Samples $(\mathrm{N} \geq 3)$ were cut from membranes that were washed and dried. The mass of the samples was determined (MBefore), and samples were then submerged in an excess of chloroform for two days. Afterwards, the samples were dried until constant weight and weighed again (MAfter). The gel content was then determined with Equation (2).

$$
\text { Gel content }=\frac{\mathrm{M}_{\text {After }}}{\mathrm{M}_{\text {Before }}} \cdot 100 \%
$$

\subsubsection{Proton Nuclear Magnetic Resonance Spectroscopy ( $\left.{ }^{1} \mathrm{H}-\mathrm{NMR}\right)$}

We performed a qualitative proton nuclear magnetic resonance spectroscopy ( $\left.{ }^{1} \mathrm{H}-\mathrm{NMR}\right)$ analysis of the sol fraction of membranes to determine whether uncrosslinked PTMC, PEO or PETA, as well as residual Irgacure 2959, hexanol or chloroform, were still present in washed membranes. Samples of the membranes $(N \geq 5)$ were cut and placed in deuterated chloroform as a solvent at a concentration of 15 $\mathrm{mg} / \mathrm{mL}$. Samples containing $2.5 \mathrm{mg} / \mathrm{mL}$ of one of the pure components were used as references $(\mathrm{N}=3)$. The samples were then shaken gently for two days in the dark. Afterwards, in the case of the membranes, the membrane samples were taken out of the chloroform. The chloroform (including any present molecules) as well as the reference samples, were then added to NMR tubes (Sigma-Aldrich, Darmstadt, Germany, cat. Z274526-1PAK, Wilmad 527-PP-8) for analysis in a Bruker Ascend 400 (Avance III $400 \mathrm{MHz}$ NMR spectrometer) (Bruker, Billerica, MA, USA).

\subsection{Mechanical properties of membranes}

The E-modulus of membranes was determined by placing dry dumbbellshaped samples ( $50 \mathrm{~mm} \times 4 \mathrm{~mm}$ ) in a Zwick/Roell Z020/SND tensile tester (Zwick/Roell, Ulm, Germany) with a $500 \mathrm{~N}$ load cell and a grip-to-grip separation of $30 \mathrm{~mm}$. Stress-strain measurements were then done by applying a preload of $0.01 \mathrm{~N}$, followed by elongation of the samples at a speed of $10 \mathrm{~mm} / \mathrm{min}$. at $19-20{ }^{\circ} \mathrm{C}$. The strain was measured with 
extensometers. The E-moduli were then determined as the slope of the initial linear phase of the stress-strain curves $(\mathrm{N} \geq 2$ from different membranes).

\subsection{Porosity, morphology and water transport}

\subsubsection{Overall porosity and thickness}

Porosity of the membranes was measured by punching circular samples with a diameter of $26 \mathrm{~mm}$ of membranes after washing and drying. Samples from different membranes were used $(N \geq 4)$. Thickness $(N \geq 6)$ of the samples was then measured with a calliper (Model ID-C112B, Mitutoyo Corp., Kawasaki, Japan). Subsequently, the porosity was calculated with Equations 3-6:

$$
\text { Porosity }=\frac{\text { total sample volume }- \text { skeletal volume }}{\text { total sample volume }} \cdot 100 \%
$$

The total sample volume and skeletal volume were determined by:

Total sample volume $=\pi \cdot r^{2}$ of sample $\cdot$ thickness of sample

Skeletal volume $=\frac{\text { weight of sample }}{\text { overall density of membrane }}$

Overall density of membrane $=(86.33 \% \cdot$ density of PTMC $)+$ $(8.63 \% \cdot$ density of PEO $)+(5.04 \% \cdot$ density of PETA $)$

The skeletal volume was determined with Equation (5). For the overall density of the membranes, it was assumed that there was a complete removal of chloroform, Irgacure and hexanol, while all of the initial PTMC, PEO and PETA was still present in the membranes. Thus, the percentages in Equation (6) represent the wt $\%$ of PTMC, PEO and PETA, respectively, in the membranes after removal of chloroform, Irgacure and hexanol. Densities of PTMC, PEO and PETA are $1.31 \mathrm{~g} / \mathrm{mL}, 1.21 \mathrm{~g} / \mathrm{mL}$ and $1.18 \mathrm{~g} / \mathrm{mL}$, respectively. 


\subsubsection{Membrane morphology}

Membrane morphology was assessed by Scanning Electron Microscopy (SEM). Dry samples of the membranes were gold-sputtered under vacuum in a sputter coater (Cressington 108 Auto, Cressington, Watford, UK) equipped with a pure gold target (Aurion, Wageningen, The Netherlands, cat. 91017-AU) at $10 \mathrm{~mA}$ for $60 \mathrm{~s}$. Subsequent imaging was performed on a JSM-6010LA (JEOL, Tokyo, Japan) at $5 \mathrm{kV}$. Pictures of M0M3 membranes $(N \geq 7)$ taken from membranes cast on different days were analysed with ImageJ for pore analysis. First, the average pore diameter was calculated for each pore, after which these values were used to determine the average pore diameter \pm SD.

\subsubsection{Water transport across the membranes}

Circular samples of membranes with a diameter of $26 \mathrm{~mm}$ were punched. PET membranes with $0.4 \mu \mathrm{m}$ pores (Sigma-Aldrich, Darmstadt, Germany, Corning, cat. CLS3450) and PC membranes with $0.4 \mu \mathrm{m}$ pores (Merck Millipore, Darmstadt, Germany, Millicell, cat. PIHP03050) from commercial inserts were cut out of their inserts, in the following referred to as PET0.4 and PC0.4, respectively. Samples $(\mathrm{N} \geq 3)$ were taken from different membranes that were cast on multiple days. According to standard protocol, the membranes were prewetted overnight in $100 \%$ ethanol, after which they were stabilised by placing them in an Amicon cell (Merck Millipore, Darmstadt, Germany, Amicon cell 8003). The PET membranes were used without prewetting with ethanol since they performed better this way. A transmembrane pressure (TMP) of 1 bar for M0, M1 and PET0.4 samples, and 0.3 bar for M2, M3 and PC0.4 samples was then applied. Afterwards, the pressure was lowered, and the amount of permeated water was measured at different TMP, i.e. 0.3, 0.5 and 1 bar for M0, M1 and PET0.4 samples, and 0.1, 0.2 and 0.3 bar for M2, M3 and PC0.4 samples. The flux of the latter membranes was difficult to measure at higher TMP due to the high flow through these membranes. Measurements were performed for at least $15 \mathrm{~min}$. for each TMP. Water permeance was then determined as the slope of the best linear fit of the flux versus TMP for each sample. 


\subsection{Statistical analysis}

Statistical analysis was done with a One-way-ANOVA with a Bonferroni post-hoc test in Graphpad Prism5 software (Graphpad, San Diego, CA, USA) with $\mathrm{P}<0.05$. Capped lines with asterisks mark significant differences in graphs.

\section{Results and discussion}

\subsection{Membrane fabrication}

For the membrane fabrication, the basic polymer dope contained PTMC, chloroform, PETA, Irgacure 2959, PEO and a non-solvent. We first optimised the polymer dope composition and EIPS conditions for the fabrication of porous PTMC membranes.

\subsubsection{PEO and non-solvent type}

Based on earlier studies on PTMC-based scaffolds [31,37], we added PEO as pore former and stabiliser in the PTMC dope, which led to porous membranes (results not shown). Moreover, PEO improved membrane handling by making the membranes less sticky. Therefore, PEO was included in all polymer dopes at a PTMC to PEO ratio of 1:0.1 w/w following Papenburg et al. [31].

We also investigated the effect of adding various non-solvents in the PTMC polymer dope for the production of porous PTMC membranes. For this, we prepared polymer dopes with PTMC (MW $=1,300 \mathrm{~kg} / \mathrm{mol})$ and different alcohols as non-solvents, i.e. ethanol, propanol, butanol or hexanol. Figure 1 shows SEM images of the air side (i.e. the side facing the ambient air where most of the chloroform evaporates) and of the crosssection of the produced membranes. The application of small, volatile alcohols, i.e. ethanol and propanol, as non-solvents mainly resulted in non-porous membranes, except for a few superficial 'pores' in the case of propanol. The application of butanol resulted in membranes with shallow pore-like structures on the air side only, while the other parts of the membranes were non-porous. The application of hexanol, however, resulted in porous PTMC membranes, at the surface and across the 
membrane (Figure 1). This is likely because the less volatile hexanol remained present in the entire cast polymer layer, allowing proper coalescence, which resulted in pore formation throughout the entire membrane.

These results are in agreement with other studies which highlighted the need for low non-solvent evaporation for achieving porous membranes via EIPS [44,45]. Pervin et al. showed that when the non-solvent evaporation is increased at high temperature, EIPS resulted in membranes with only surface pores [45]. Non-solvent evaporation and subsequent diffusion to the air side likely also occurred during the fabrication of our membranes, mainly with butanol, propanol and ethanol. This was stimulated on top of the PTMC membranes by the airflow applied across the surface. Despite evaporation, the concentration of butanol at the air side was high enough to result in limited pore formation. Residual amounts of the more volatile propanol and ethanol were probably too small for EIPS to occur. Based on these results, hexanol was used as the non-solvent in further membrane fabrication. 
- Chapter 5 -

Porous and flexible PTMC membranes fabricated by EIPS

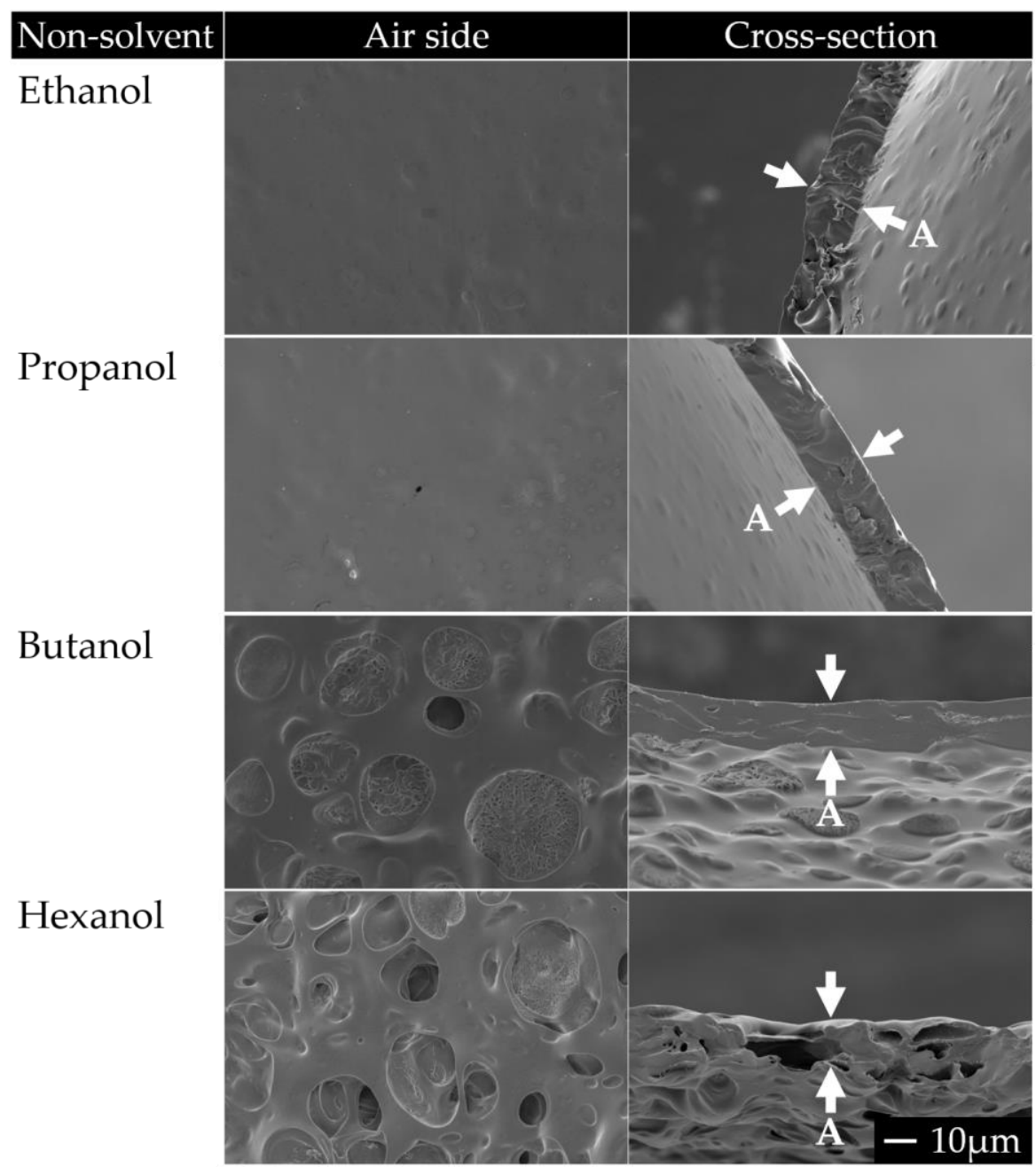

Figure 1. SEM images of membranes made from PTMC and different alcohols in the polymer dope as non-solvents. The ratio of PTMC to non-solvent was 1:1 $(w / w)$. Magnification: 1,000x. Scale bar applies to all images. The cross-section of the membranes is shown between arrows. An ' $\mathrm{A}$ ' adjacent to an arrow marks the air side of the membrane. Images are representative of observations of multiple membranes. 


\subsubsection{Ambient humidity and substrate temperature}

We also performed preliminary studies on the effect of humidity and temperature of the casting plates on the morphology of the PTMC membranes. Membranes with $3 \mathrm{wt} \% 1,300 \mathrm{~kg} / \mathrm{mol} \mathrm{PTMC}$ and $3 \mathrm{wt} \%$ hexanol were cast on silicon wafers at $50 \%$ humidity or $60 \%$ humidity, the latter created by a humidifier. Afterwards, the cast membranes and silicon wafers were placed on glass plates of $19-20{ }^{\circ} \mathrm{C}$ or $-25^{\circ} \mathrm{C}$.

We found that membrane porosity increased both at relatively high ambient humidity of $60 \%$ during EIPS and by placement on the cold glass plates, as well as both combined (results not shown). These conditions likely hindered chloroform evaporation, thus slowing down EIPS and allowing more time for hexanol to coalesce, resulting in larger pore size and higher porosity of the membranes, consistent with other studies $[44,45]$. Furthermore, evaporation of the solvent and possibly non-solvent leads to a decrease in the temperature of the air side, also known as the evaporative cooling effect [45]. This stimulates water deposition from the ambient air onto the membrane surface. This water deposition can already lead to pores at the air side [45] and is the main principle of pore formation in (water-)vapour-induced phase separation (VIPS) [46-48]. Higher humidity of the ambient air could promote this effect, as was shown by Pervin et al. [45]. Thus, high ambient humidity, as well as placement on cold glass plates during casting and EIPS, were used in further membrane fabrication.

\subsubsection{PTMC molecular weight}

Subsequently, we investigated the effect of the MW of PTMC on the fabrication of porous membranes. Polymer dopes were prepared consisting of the basic components mentioned previously, with $3 \mathrm{wt} \%$ hexanol as non-solvent and PTMC with a MW of either $1,300 \mathrm{~kg} / \mathrm{mol}$ or $600 \mathrm{~kg} / \mathrm{mol}$ (Table 1). We then cast membranes on wafers and performed EIPS in a humid environment on cold plates as described above. The polymer dopes with PTMC of $1,300 \mathrm{~kg} / \mathrm{mol}$ were quite viscous, and the PTMC was incompletely dissolved, resulting in heterogeneous membranes (Figure S1). In contrast, the PTMC with a MW of $600 \mathrm{~kg} / \mathrm{mol}$ 
could be dissolved well, and the produced membranes were homogeneous. Besides, these membranes had larger pores and higher porosity. The coalescence of hexanol was likely better due to the lower viscosity of the polymer dope and thus higher molecular mobility, similar to the results reported by Zhao et al. [44].

Pervin et al. investigated the formation of porous poly(methyl methacrylate) (PMMA) membranes via EIPS and applied PMMA with a MW of $15 \mathrm{~kg} / \mathrm{mol}$ and $120 \mathrm{~kg} / \mathrm{mol}$ and mixtures of both. They reported that application of $15 \mathrm{~kg} / \mathrm{mol}$ PMMA resulted in non-porous membranes [45]. In contrast, they found pore formation when the PMMA mixtures were used. Moreover, higher amounts of $120 \mathrm{~kg} / \mathrm{mol}$ PMMA led to pore formation deeper in the membranes. They suggested that the $15 \mathrm{~kg} / \mathrm{mol}$ PMMA was too soluble, and thus EIPS was only initiated in the presence of the $120 \mathrm{~kg} / \mathrm{mol}$ PMMA and when the concentration of $120 \mathrm{~kg} / \mathrm{mol}$ PMMA was sufficiently high.

They also reported that polymer dopes with more than $40 \mathrm{wt} \%$ PMMA had a high viscosity, which prevented homogeneous mixing leading to heterogeneous polymer dopes [45]. This is consistent with our findings with the high MW PTMC $(1,300 \mathrm{~kg} / \mathrm{mol})$, where the resulting PTMC membranes were heterogeneous due to the high polymer dope viscosity. The PTMC concentration in our polymer solutions follows Papenburg et al. [31]. Lower PTMC concentrations, as used in studies with relatively thick PTMC scaffolds $[49,50]$, may decrease heterogeneity in the case of the $1,300 \mathrm{~kg} / \mathrm{mol}$ PTMC, but will likely also result in very thin and fragile membranes. Moreover, Pervin et al. showed that a very low polymer concentration could limit the occurrence of EIPS [45]. Conversely, increasing the polymer concentration in the case of the $600 \mathrm{~kg} / \mathrm{mol}$ PTMC would likely result in denser membranes and similar problems as with $1,300 \mathrm{~kg} / \mathrm{mol}$ PTMC due to a too high viscosity of the polymer solution. Therefore, the PTMC concentration in the polymer solutions was kept at $3 \mathrm{wt} \%$.

Based on these results, the MW of the polymer needs careful consideration for obtaining porous and homogeneous membranes via EIPS. Application of low MW polymer (having high solubility and low 
viscosity) can lead to non-porous membranes, while application of high MW polymer (having low solubility and high viscosity) can lead to nonhomogeneous porous membranes. Based on our results, the PTMC with a MW of $600 \mathrm{~kg} / \mathrm{mol}$ is the most suitable for obtaining porous membranes. Therefore, it was used in further experiments.

\subsection{The effect of the non-solvent amount}

Here, we prepared polymer dopes containing PTMC of $600 \mathrm{~kg} / \mathrm{mol}$ and different amounts of hexanol to tailor the membrane properties. Membranes are referred to as M0-M3 based on the ratio of PTMC to hexanol in the polymer dope, i.e. 1:0 to 1:3 $(\mathrm{w} / \mathrm{w})$, respectively (see also in Section 2.3 and Table 2).

\subsubsection{The polymer network in the membranes}

Since optimal membrane crosslinking is desired for achieving stable porous PTMC membranes, we assessed the polymer network integrity of the developed membranes. Table 2 presents the gel content of the membranes, which expresses the percentage of the membranes that was crosslinked. Increasing amounts of hexanol in the polymer dope resulted in membranes with lower gel content, i.e. an average gel content of $95.9 \%$ to $52.9 \%$ for $\mathrm{M} 0-\mathrm{M} 3$.

Table 2. PTMC membrane properties as a result of different hexanol amounts in the polymer dopes. Polymer dopes were made using chloroform as a solvent. Dopes always contained PTMC with a MW of $600 \mathrm{~kg} / \mathrm{mol}$, PEO, PETA and Irgacure 2959. The remaining part of the polymer dope either consisted solely of chloroform or an amount of hexanol, complemented by chloroform (see $2^{\text {nd }}$ and $3^{\text {rd }}$ rows). The table shows different membrane properties and the effect of hexanol content in the polymer dope on these properties. Data were analysed by One-way ANOVA. Significant differences $(\mathrm{P}<0.05)$ with other membranes are marked by (M0-M3) underneath the data referring to those membranes. Significant differences in pore size between parts of a specific membrane are marked by (A), (S) or (Cr), indicating air side, substrate side or cross-section, respectively. Pore size analysis was based on $\geq 7$ images per condition. 


\section{- Chapter 5 -}

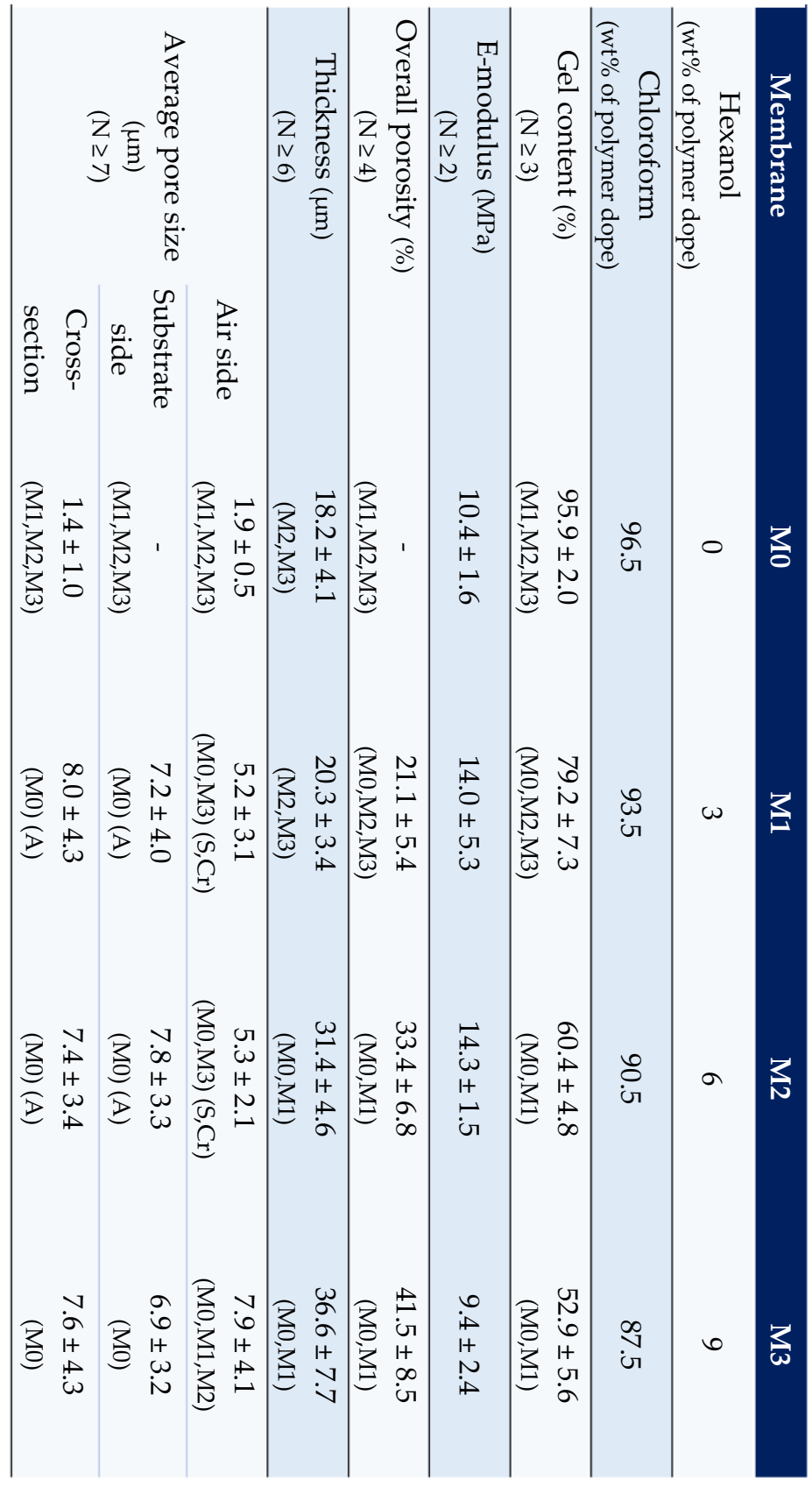


The lower gel content could be due to the partial dissolution of the crosslinking agents in the hexanol, which limited their availability and thus decreased crosslinking efficiency and gel content. Higher hexanol content in the polymer dope probably increased this effect. However, due to the relatively low amounts of non-solvent, the gel content after EIPS and crosslinking was still high enough to provide membranes with good integrity. In fact, the M1-M3 membranes could be handled well when placed in cell culture liquids, i.e. PBS and cell culture media (results not shown). Besides, these membranes could be wetted easily, but did not swell significantly in these liquids and were not adhesive.

${ }^{1} \mathrm{H}-\mathrm{NMR}$ analysis of the sol fraction of membranes indicates that uncrosslinked PTMC and PEO were present in the membranes (Figure S2). However, there was no detectable hexanol or chloroform (Figure S3), nor Irgacure 2959 and PETA (Figure S4), suggesting that they were removed during washing or, in the case of PETA, crosslinked in the membranes. PTMC and PEO were likely both crosslinked through hydrogen abstraction [32,51]. Together with multifunctional acrylic radicals from the PETA [32], this resulted in a network of PETA, PEO and PTMC. Uncrosslinked PTMC and PEO were likely entangled in the polymer network due to their high MW and could not be removed completely by washing with ethanol and water.

Similar findings of entrapped PEO in PTMC scaffolds were reported by Papenburg et al. [31] who used $266 \mathrm{~kg} / \mathrm{mol}$ PTMC and PEO of 6,000 $\mathrm{kg} / \mathrm{mol}$ to fabricate porous PTMC scaffolds by LIPS. After washing the scaffolds in MilliQ water, there was still PEO present. They proposed that during phase separation, the viscosity of the PTMC-rich phase increased to a point where some of the PEO could be entrapped in the PTMC phase. The viscosity of our polymer dopes was likely higher since our PTMC had a substantially higher MW, while the concentration and MW of PEO were similar. Moreover, our membranes were crosslinked for at least 52.9\%. Therefore, uncrosslinked PTMC and PEO were likely only removed when the membranes were swollen in chloroform. 


\subsubsection{Mechanical properties of membranes}

Table 2 also features the E-moduli of M0-M3 membranes which were in the range of 9-14 MPa. There was no significant difference between M0M3 membranes. This further confirmed that the decreasing gel content did not influence the stability or mechanical properties of the membranes. The E-moduli of the PTMC membranes were significantly lower than those of the previously mentioned PET and PC membranes (2-3 GPa [11] and 2-2.4 GPa [12], respectively), but similar to those of some PDMS membranes (4 $\mathrm{kPa}$ to several $\mathrm{MPa}[22]$ ).

Bat et al. [32] made films from high MW PTMC that were crosslinked similarly to our M0-M3 membranes. Those films with similar gel content and E-moduli (approximately $10 \mathrm{MPa}$ ) to our membranes had a stress at break up to more than $30 \mathrm{MPa}$, an elongation at break up to more than $600 \%$ and a permanent deformation of $2 \%$ or less. These results indicated good toughness and the capability of being used for cyclic stretch.

As established before, the mechanical properties of PTMC structures are well preserved in a wet state $[37,38]$. The E-modulus could decrease in a wet state [38], which would be beneficial for our application. Moreover, the M0-M3 membranes have low swelling, as shown by the experiments in cell culture liquids, and thus also a low water uptake. Therefore, it is unlikely that the mechanical properties of our membranes would be negatively affected by wetting.

\subsubsection{Porosity, morphology and water transport}

Membranes in both Transwell ${ }^{\circledR}$ inserts and various OOCs require sufficient porosity to allow nutrient transport to the cultured cells as well as cell-cell signalling by secreted mediators when culturing cells on either side of the membrane. Therefore, we investigated how to tailor the overall porosity, pore morphology and pore connectivity across the membranes (Table 2 and Figures 2 and 3). 


\section{Overall porosity and thickness}

The M1-M3 membranes were porous, whereas the M0 membranes were non-porous (Table 2). The hexanol concentration in the polymer dope increased overall porosity from $21.1 \%$ in M1 membranes to $33.4 \%$ in M2 membranes and $41.5 \%$ in $\mathrm{M} 3$ membranes. However, although there were differences between the M1 membranes and the M2 and M3 membranes, all porosities were relatively low. That could explain why the E-moduli did not change between the M1-M3 membranes. The M2 and M3 membranes were also thicker than the M0 membranes (Table 2) due to their porosity.

\section{Membrane morphology}

Figure 2 and Table 2 show SEM images and pore sizes, respectively, of the air side, the substrate side and the cross-sections of M0-M3 membranes. M0 membranes were mainly non-porous, with only a few pores on the air side and in the cross-section of some samples (Figure 2). The pores did not seem to interconnect due to the low pore density as was apparent by the cross-section of the M0 membranes. Moreover, the substrate sides of the samples completely lacked pores, thus preventing any interconnection between the air and substrate side of the M0 membranes. M1-M3 membranes had a spongy structure with seemingly interconnected pores and higher overall porosity and thickness (Figure 2), consistent with the results of the overall porosity and thickness (Table 2). The porosity was higher at the air side than the substrate side (Figure 2). The diffusion of chloroform towards the air side, caused by its evaporation from the surface, likely extended the duration of EIPS at the air side. This probably stimulated the coalescence of hexanol, resulting in higher porosity at the air side than the substrate side. The pore sizes on the air side of M1 and M2 membranes $(5.2 \pm 3.1$ and $5.3 \pm 2.1 \mu \mathrm{m}$, respectively) were smaller than those on the air side of M3 membranes, i.e. $7.9 \pm 4.1 \mu \mathrm{m}$ (Table 2). Thus, increasing the hexanol amount in the dope increased the surface pore size. 
- Chapter 5 -

Porous and flexible PTMC membranes fabricated by EIPS

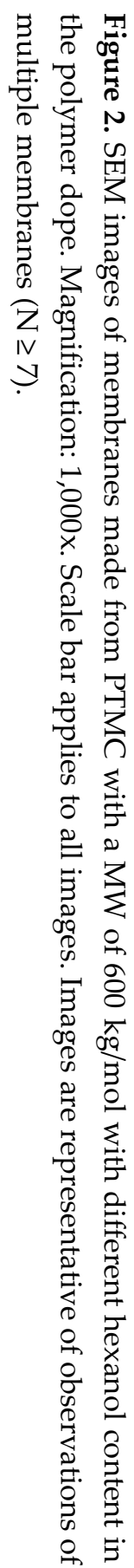

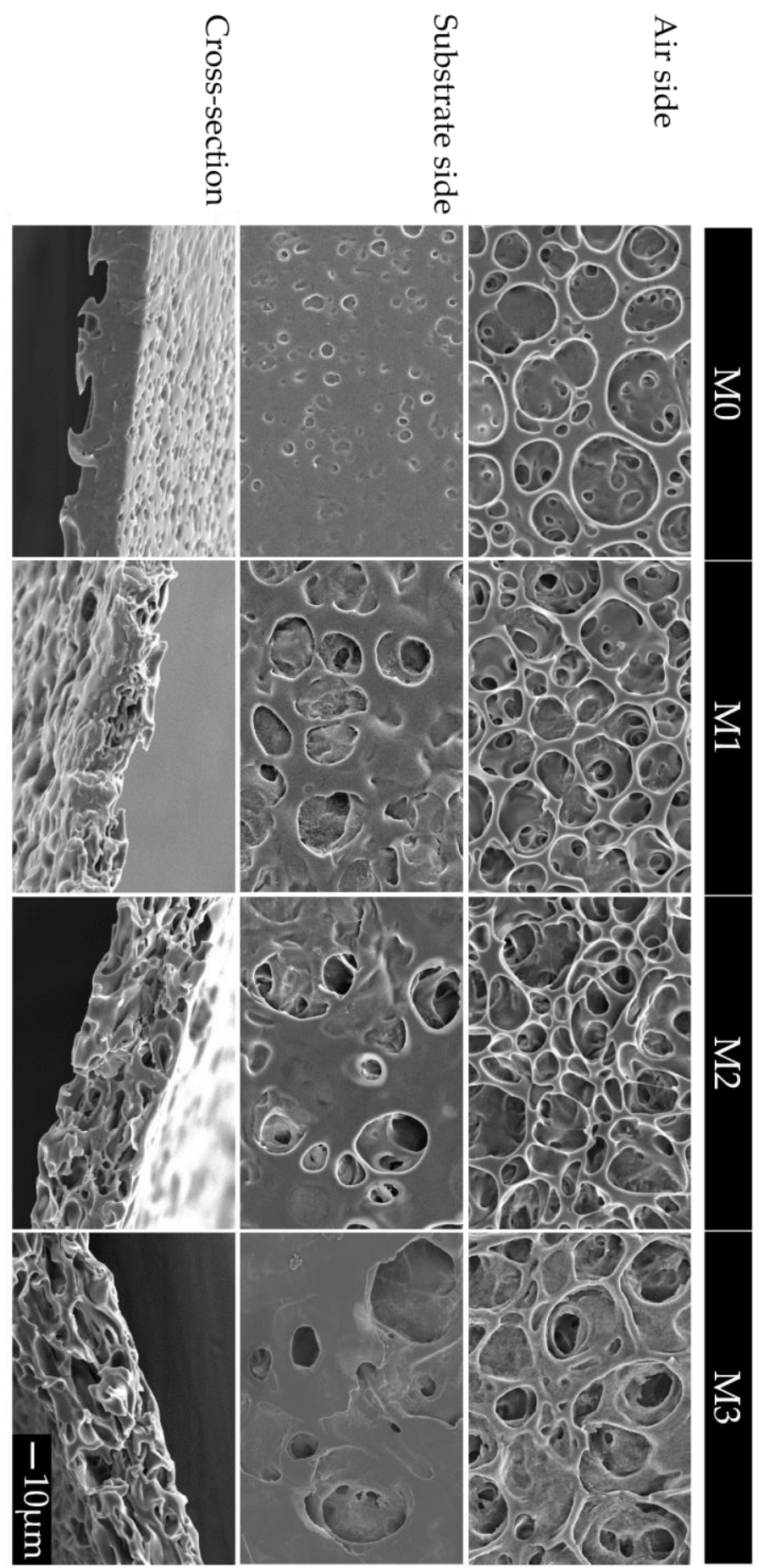


Pores on the air side of M1 and M2 membranes were smaller compared to those on their respective substrate sides and in their cross-sections. Perhaps the deposition of water droplets on the air sides of the membranes limited the coalescence of hexanol compared to the substrate sides and cross-sections, since hexanol and water do not mix well. The additional hexanol in M3 membranes may have been enough to avoid the effect of the water. Moreover, phase separation likely occurred earlier in M3 membranes than in M1 and M2 membranes because of the higher nonsolvent concentration, as was suggested by Zhao et al. [44]. Thus, EIPS started in M3 membranes when there was less deposition of the water droplets and thus less interference with coalescence compared to EIPS in the M1 and M2 membranes.

Pore shape also seemed to become less circular as the hexanol concentration was increased (Figure 2). While M1 membranes and to a lesser extent M2 membranes featured mostly circular pores, the shape of pores in M3 membranes was more irregular. The latter may have been caused by better coalescence of multiple larger hexanol droplets on the air side of M3 membranes compared to that in M1 and M2 membranes.

Consistent with the increasing pore size seen at the surface of M1 to M3 membranes, Zhao et al. showed that increasing the non-solvent concentration in the polymer dope led to larger pores and lower pore density [44]. As mentioned, they suggested that a higher non-solvent amount initiated EIPS faster. EIPS, therefore, occurred at a higher solvent concentration and thus at a lower viscosity, which can stimulate coalescence of the non-solvent. Thus, at higher non-solvent content, there are more favourable conditions for the coalescence of the non-solvent as well as more non-solvent present, which both contribute to the increased pore size.

In general, M0-M3 membranes had a relatively smooth substrate side (Figure 2), likely due to the contact with the smooth silicon wafers. In contrast, the air side of the membranes had higher roughness and contained crater-like structures. These structures resembled the 
honeycomb structures also found in membranes fabricated with VIPS and were likely caused by water deposition on the membranes [45-48] as a result of the evaporative cooling effect mentioned earlier [45]. The humid and relatively cold casting conditions and the presence of hydrophilic PEO likely stimulated deposition of condensed water droplets on the M0M3 membranes. This may also explain why the membranes featured in Figure 1 had relatively smooth air sides. Since those membranes were cast at $19-20^{\circ} \mathrm{C}$ and $50 \%$ humidity, whereas the M0-M3 membranes were cast at $-25{ }^{\circ} \mathrm{C}$ (at the start of chloroform evaporation) and $60 \%$ humidity, the deposition of water droplets was probably limited in the former case.

The M1-M3 membranes were opaque (Figure S5), but became more transparent upon wetting. The water in the pores may help light travel through the pores. Standard cell culture inserts with PC membranes with $0.4 \mu \mathrm{m}$ pores (PC0.4) had a comparable level of transparency. In any case, application of M1-M3 membranes in in vitro organ models, e.g. OOCs, is possible, where the cells can often be viewed from multiple sides under the microscope. Moreover, a live cell tracker could be used to track the cells, if necessary.

The opaqueness of M1-M3 membranes increased when the concentration of hexanol in the polymer dope was increased (Figure S5). M1 and M3 membranes were macroscopically homogeneous, while M2 membranes were heterogeneous having both transparent and opaque areas across the entire surface area. Zhao et al., who determined the phase diagrams for their silicon rubber membranes made by EIPS [44], proposed that EIPS occurred via nucleation and growth at low and high non-solvent concentration, or via spinodal decomposition at certain non-solvent concentrations in between the former. Possibly, phase separation in the case of the M1 and M3 membranes occurred through nucleation and growth, whereas in the case of M2 membranes, EIPS took place via spinodal decomposition. The unstable phase separation in the latter case is probably the reason for the heterogeneous membrane morphology of M2 membranes compared to the M1 and M3 membranes. 
Water transport across the membranes

Figure 3 shows the water flux (Figure $3 \mathrm{~A}, \mathrm{~B}$ ) and the estimated water permeance (Figure 3C) of M0-M3 membranes compared to PC0.4 and PET membranes with $0.4 \mu \mathrm{m}$ pores (PET0.4). As expected, the non-porous M0 membranes had no water flux. However, M1-M3 membranes had high water permeance in the microfiltration range, i.e. $17,000-41,000$ $\mathrm{L} /\left(\mathrm{m}^{2} \cdot \mathrm{h} \cdot \mathrm{bar}\right)$, which confirmed that the pores were well interconnected. The average fluxes of M2 and M3 membranes were similar. These membranes already showed a water flux at a TMP of 0 bar due to the hydrostatic pressure of the water. Furthermore, water flux increased linearly with TMP in M1-M3 (Figure 3), indicating no membrane compaction and good stability of the porous networks.

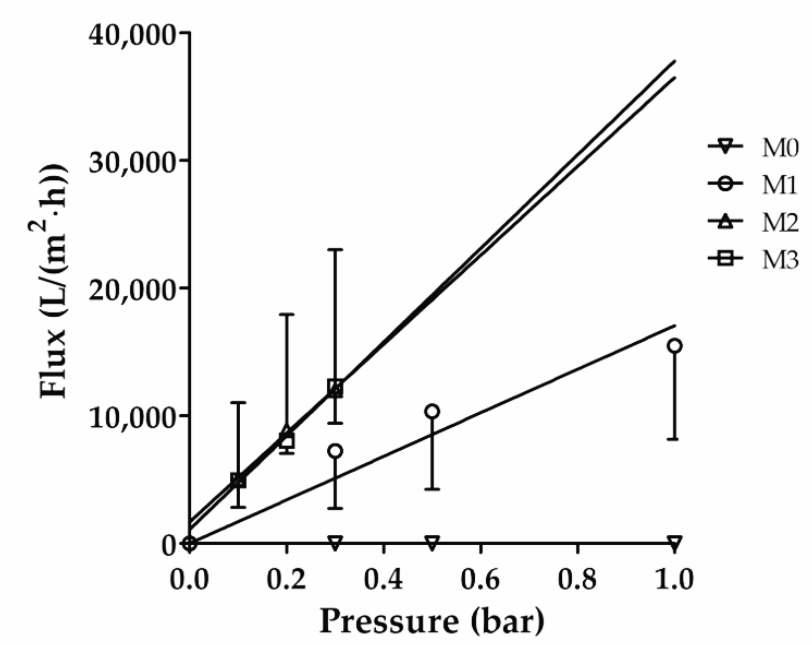

(A)

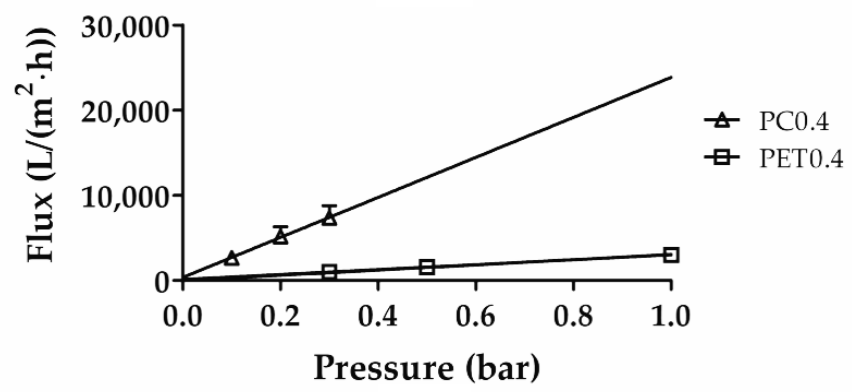

(B) 


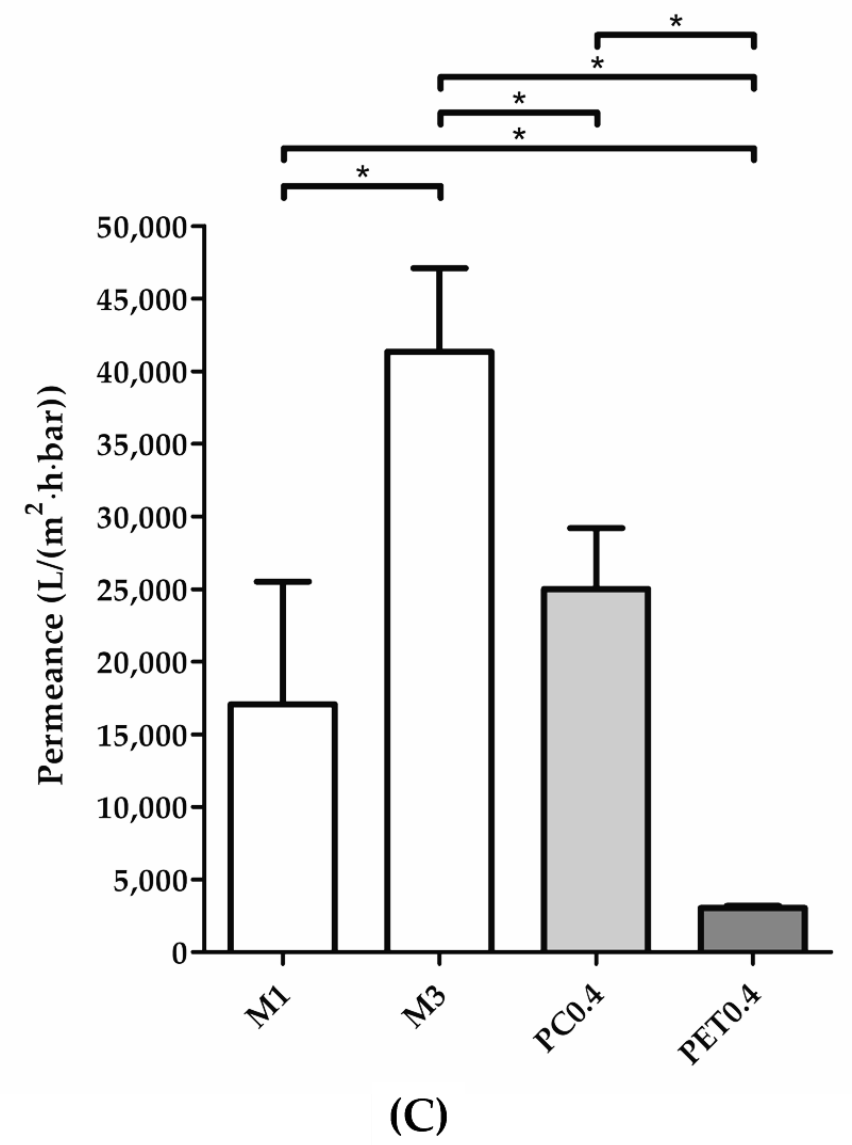

Figure 3. Effect of the hexanol amount on the water transport across M0-M3 PTMC membranes. PC and PET membranes with $0.4 \mu \mathrm{m}$ pores taken from commercial inserts (PC0.4 and PET0.4, respectively) were used as a reference. $(A, B)$ Water flux of membranes measured at different TMP after pre-compaction. M2, M3 and PC0.4 samples were measured at lower pressures since their flux was too high to collect at higher pressures. Shown are average fluxes $\pm \mathrm{SD}(\mathrm{N} \geq$ 3), lines represent the best linear fit. (A) Demonstrates M0-M3, (B) shows PC0.4 and PET0.4 samples. (C) The permeance of the different membranes was calculated by determining the best linear fit of the water flux data for each sample and calculating the average. M0 membranes are not shown since they were not permeable to water. Depicted are averages $\pm S D(N \geq 3)$. Capped lines with asterisks above the graph show significant differences $(\mathrm{P}<0.05)$. M2 membranes were not included in the statistical analysis due to the high variation of their water fluxes. 
The water fluxes of the M2 membranes had a larger variation than those of M1 and M3 membranes indicating a larger variation in the pore interconnectivity of the M2 membranes, consistent with the observations made earlier about membrane heterogeneity. Due to this high variation, M2 membrane water transport data were excluded from the statistical analysis of the estimation of the water permeance of the different membranes. For comparison, the PET0.4 membranes had much lower water permeance than M1 and M3 membranes, whereas the PC0.4 membranes had a permeance similar to the M1 membranes. Besides, the permeance of the M3 membranes was higher than that of the M1 and PC0.4 membranes.

\subsection{EIPS and photo-crosslinking for tailoring of membrane properties}

In this study, we combined for the first time EIPS and photo-crosslinking to produce form-stable, porous and permeable PTMC membranes. Our method could be an interesting option to fabricate porous polymer structures from amorphous polymers. The membranes had similar porosity, pore size and thickness as LIPS-based PTMC membranes [31]. In general, the membrane pore sizes were comparable to those of PDMS membranes used in some OOCs $[1,2,10]$. LIPS, although well-known, simple and used for many applications, is less favourable for the purposes mentioned above, since polymer crosslinking via gamma-irradiation can be difficult [31] and photo-crosslinking is likely not straightforward.

Here, we prepared mechanically stable porous membranes with very good porosity, even at relatively low gel contents of $52.9 \%$ to $79.2 \%$, due to the entanglement of the PTMC and PEO. However, if lower MW polymers would be used, the obtained gel contents may be too low for proper membrane integrity since there would be less entanglement of the polymers. In those cases, it would be relevant to raise the crosslinking efficiency, which can be achieved by adding more crosslinking agents to the polymer dope [32].

Moreover, EIPS and photo-crosslinking could be used with other amorphous or semi-crystalline polymers and copolymers (e.g. TMC and 
$\varepsilon$-caprolactone ( $\varepsilon$-CL) [52] or poly(DL-lactic acid) (PDLLA) and $\varepsilon$-CL [53]) with good cell adhesive properties to develop porous membranes. In this study, we were able to make membranes with high water permeance (in the microfiltration range, i.e. $17,000-41,000 \mathrm{~L} /\left(\mathrm{m}^{2} \cdot \mathrm{h} \cdot \mathrm{bar}\right)$ similar to that of commercial PC inserts. Thus, our membranes are likely well-suited for application in cell culture systems and OOCs, allowing nutrient delivery and paracrine signalling of cells on opposite sides of the membranes. Permeance was raised by increasing the non-solvent concentration in the polymer dope. This effect was likely due to better pore interconnectivity since raising the non-solvent concentration increased overall porosity and pore size on the air side of membranes. Moreover, the permeances were high for membranes with a rather low porosity of $21.1-41.5 \%$. The relatively low porosities and pore sizes of our membranes may stimulate cells to grow in proper confluent cell layers and thus create better barriers compared to membranes or scaffolds with much higher porosities and pore sizes [32,43,44], which may discourage cell-cell contact.

The easy wetting and high permeances of the M1-M3 membranes suggest that these membranes are quite hydrophilic, which could be partially due to the presence of hydrophilic PEO at the surface. However, since the membrane surface also contains hydrophobic PTMC, the hydrophilicity is likely moderate. This is advantageous for cell culturing since moderate hydrophilicity has been shown to promote cell adhesion, due to good adsorption of cell adhesive proteins [1].

The PTMC membranes developed here have E-moduli of approximately $10 \mathrm{MPa}$, which are much lower than the E-moduli of commonly used materials such as PET and PC and similar to those of several tissues $[15,17,18]$. This is an important step forward in the development of tissuemimicking supports for cell culturing. In the future, the E-moduli could be further decreased by the use of lower PETA concentrations [29] or a diacrylate instead of a triacrylate, such as PETA [39]. Besides, EIPS and photo-crosslinking could be used with copolymers of e.g. TMC and $\varepsilon-\mathrm{CL}$, since networks of these copolymers have lower E-moduli than networks of either polymer alone [52]. 


\section{Conclusions}

Organ models often use culture membranes with properties that do not match those of the represented tissue. Methods to create membranes with suitable pore sizes from flexible but low cell adhesive PDMS are often elaborate, while more easily applicable methods often require stiff and/or low cell adhesive materials. This study shows, for the first time, the development of porous, form-stable PTMC membranes by combining EIPS and photo-crosslinking. Important parameters for tailoring the membrane fabrication, such as polymer dope additives, humidity, and the type and amount of non-solvent were investigated. We could adapt the membrane pore size on the air side and the pore connectivity by increasing the non-solvent content in the polymer dope. The resulting M1 and M3 membranes both had high water permeance similar or higher than that of commercial membranes, good mechanical properties and suitable pore sizes for application in in vitro organ models. These characteristics make both the M1 and M3 membranes promising as cell culture supports for use in such models. Moreover, the obtained understanding of EIPS and its established compatibility with photocrosslinking could be used to prepare membranes from other amorphous or semi-crystalline polymers for biomedical applications.

\section{References}

1. Pasman, T.; Grijpma, D.; Stamatialis, D.; Poot, A. Flat and microstructured polymeric membranes in organs-on-chips. J. R. Soc. Interface. 2018, 15. DOI: 10.1098/rsif.2018.0351.

2. Huh, D.; Kim, H.J.; Fraser, J.P.; Shea, D.E.; Khan, M.; Bahinski, A.; Hamilton, G.A.; Ingber, D.E. Microfabrication of human organs-on-chips. Nat. Protoc. 2013, 8, 2135-2157. DOI: 10.1038/nprot.2013.137.

3. Huh, D.; Hamilton, G.A.; Ingber, D.E. From 3D cell culture to organs-on-chips. Trends Cell Biol. 2011, 21, 745-754. DOI: 10.1016/j.tcb.2011.09.005.

4. Huh, D.; Leslie, D.C.; Matthews, B.D.; Fraser, J.P.; Jurek, S.; Hamilton, G.A.; Thorneloe, K.S.; McAlexander, M.A.; Ingber, D.E. A human disease model of drug toxicity-induced pulmonary edema in a lung-on-a-chip microdevice. Sci. Transl. Med. 2012, 4. DOI: 10.1126/scitranslmed.3004249. 
5. Huh, D.; Matthews, B.D.; Mammoto, A.; Montoya-Zavala, M.; Hsin, H.Y.; Ingber, D.E. Reconstituting organ-level lung functions on a chip. Science. 2010, 328, 16621668. DOI: 10.1126/science.1188302.

6. Huh, D.; Torisawa, Y.S.; Hamilton, G.A.; Kim, H.J.; Ingber, D.E. Microengineered physiological biomimicry: Organs-on-chips. Lab Chip. 2012, 12, 2156-2164. DOI: 10.1039/c2lc40089h.

7. Kim, H.J.; Huh, D.; Hamilton, G.A.; Ingber, D.E. Human gut-on-a-chip inhabited by microbial flora that experiences intestinal peristalsis-like motions and flow. Lab Chip. 2012, 12, 2165-2174. DOI: 10.1039/c2lc40074j.

8. Kim, H.J.; Ingber, D.E. Gut-on-a-chip microenvironment induces human intestinal cells to undergo villus differentiation. Integr. Biol. 2013, 5, 1130-1140. DOI: 10.1039/c3ib40126j.

9. Punde, T.H.; Wu, W.H.; Lien, P.C.; Chang, Y.L.; Kuo, P.H.; Chang, M.D.T.; Lee, K.Y.; Huang, C.D.; Kuo, H.P.; Chan, Y.F.; et al. A biologically inspired lung-ona-chip device for the study of protein-induced lung inflammation. Integr. Biol. 2015, 7, 162-169. DOI: 10.1039/c4ib00239c.

10. Stucki, A.O.; Stucki, J.D.; Hall, S.R.; Felder, M.; Mermoud, Y.; Schmid, R.A.; Geiser, T.; Guenat, O.T. A lung-on-a-chip array with an integrated bio-inspired respiration mechanism. Lab Chip. 2015, 15, 1302-1310. DOI: 10.1039/c4lc01252f.

11. Gotoh, K.; Yasukawa, A.; Taniguchi, K. Water contact angles on poly(ethylene terephthalate) film exposed to atmospheric pressure plasma. J. Adhes. Sci. Technol. 2011, 25, 307-322. DOI: 10.1163/016942410X511114.

12. Lee, J.H.; Lee, S.J.; Khang, G.; Lee, H.B. Interaction of fibroblasts on polycarbonate membrane surfaces with different micropore sizes and hydrophilicity. J Biomater. Sci. Polym. Ed. 1999, 10, 283-294. DOI: 10.1163/156856299X00351.

13. Cowin, S.C.; Van Buskirk, W.C.; Ashman, R.B. Properties of bone. In Handbook of Bioengineering, 1st ed.; Skalak, R., Chien, S., Eds.; McGraw-Hill: New York, NY, USA, 1987; pp. 2.1-2.27.

14. Hunt, K.D.; O'Loughlin, V.D.; Fitting, D.W.; Adler, L. Ultrasonic determination of the elastic modulus of human cortical bone. Med. Biol. Eng. Comput. 1998, 36, 51-56. DOI: 10.1007/BF02522857.

15. Bose, S.; Roy, M.; Bandyopadhyay, A. Recent advances in bone tissue engineering scaffolds. Trends Biotechnol. 2012, 30, 546-554. DOI: 10.1016/j.tibtech.2012.07.005.

16. Lai, Y.S.; Chen, W.C.; Huang, C.H.; Cheng, C.K.; Chan, K.K.; Chang, T.K. The effect of graft strength on knee laxity and graft in-situ forces after posterior cruciate ligament reconstruction. PLoS ONE. 2015, 10. DOI: 10.1371/journal.pone.0127293.

17. Bat, E.; Zhang, Z.; Feijen, J.; Grijpma, D.W.; Poot, A.A. Biodegradable elastomers for biomedical applications and regenerative medicine. Regen. Med. 2014, 9, 385398. DOI: 10.2217/RME.14.4.

18. Terzini, M.; Bignardi, C.; Castagnoli, C.; Cambieri, I.; Zanetti, E.M.; Audenino, A.L. Ex Vivo Dermis Mechanical Behavior in Relation to Decellularization 
Treatment Length. Open Biomed. Eng. J. 2016, 10, 34-42. DOI: $10.2174 / 1874120701610010034$.

19. Butcher, D.T.; Alliston, T.; Weaver, V.M. A tense situation: Forcing tumour progression. Nat. Rev. Cancer. 2009, 9, 108-122. DOI: 10.1038/nrc2544.

20. Chen, Y.F.; Chan, H.N.; Michael, S.A.; Shen, Y.S.; Chen, Y.; Tian, Q.; Huang, L.; $\mathrm{Wu}, \mathrm{H}$. A microfluidic circulatory system integrated with capillary-assisted pressure sensors. Lab Chip. 2017, 17, 653-662. DOI: 10.1039/c6lc01427e.

21. Di Caprio, G.; Stokes, C.; Higgins, J.M.; Schonbrun, E. Single-cell measurement of red blood cell oxygen affinity. Proc. Natl. Acad. Sci. USA. 2015, 112, 9984-9989. DOI: $10.1073 /$ pnas.1509252112.

22. Ochsner, M.; Dusseiller, M.R.; Grandin, H.M.; Luna-Morris, S.; Textor, M.; Vogel, V.; Smith, M.L. Micro-well arrays for 3D shape control and high resolution analysis of single cells. Lab Chip. 2007, 7, 1074-1077. DOI: 10.1039/b704449f.

23. Schüller-Ravoo, S.; Papenburg, B.; Stamatialis, D.; Feijen, J.; Grijpma, D. Microstructured Photo-Crosslinked Poly(Trimethylene Carbonate) for Use in Soft Lithography Applications: A Biodegradable Alternative for Poly(Dimethylsiloxane). ChemPhysChem. 2018, 19, 2085-2092. DOI: 10.1002/cphc.201701308.

24. Moraes, C.; Kagoma, Y.K.; Beca, B.M.; Tonelli-Zasarsky, R.L.; Sun, Y.; Simmons, C.A. Integrating polyurethane culture substrates into poly(dimethylsiloxane) microdevices. Biomaterials. 2009, 30, 5241-5250. DOI: 10.1016/j.biomaterials.2009.05.066.

25. Le-The, H.; Tibbe, M.; Loessberg-Zahl, J.; Palma do Carmo, M.; van der Helm, M.; Bomer, J.; van den Berg, A.; Leferink, A.; Segerink, L.; Eijkel, J. Large-scale fabrication of free-standing and sub- $\mu \mathrm{m}$ PDMS through-hole membranes. Nanoscale. 2018, 10, 7711-7718. DOI: 10.1039/c7nr09658e.

26. Wala, J.; Maji, D.; Das, S. Influence of physico-mechanical properties of elastomeric material for different cell growth. Biomed. Mater. 2017, 12. DOI: 10.1088/1748-605X/aa7e81.

27. Toepke, M.W.; Beebe, D.J. PDMS absorption of small molecules and consequences in microfluidic applications. Lab Chip. 2006, 6, 1484-1486. DOI: 10.1039/b612140c.

28. Van Meer, B.J.; de Vries, H.; Firth, K.; van Weerd, J.; Tertoolen, L.; Karperien, H.; Jonkheijm, P.; Denning, C.; IJzerman, A.P.; Mummery, C.L. Small molecule absorption by PDMS in the context of drug response bioassays. Biochem. Biophys. Res. Commun. 2017, 482, 323-328. DOI: 10.1016/j.bbrc.2016.11.062.

29. Bat, E.; Feijen, J.; Grijpma, D.W. Biodegradable elastomeric networks: Highly efficient cross-linking of poly(trimethylene carbonate) by gamma irradiation in the presence of pentaerythritol triacrylate. Biomacromolecules. 2010, 11, 26922699. DOI: $10.1021 / \mathrm{bm} 1007234$.

30. Pêgo, A.P.; Siebum, B.; Van Luyn, M.J.; Gallego y Van Seijen, X.J.; Poot, A.A.; Grijpma, D.W.; Feijen, J. Preparation of degradable porous structures based on 1,3-trimethylene carbonate and D,L-lactide (co)polymers for heart tissue engineering. Tissue Eng. 2003, 9, 981-994. DOI: 10.1089/107632703322495628. 
31. Papenburg, B.J.; Schuller-Ravoo, S.; Bolhuis-Versteeg, L.A.M.; Hartsuiker, L.; Grijpma, D.W.; Feijen, J.; Wessling, M.; Stamatialis, D. Designing porosity and topography of poly(1,3-trimethylene carbonate) scaffolds. Acta Biomater. 2009, 5, 3281-3294. DOI: 10.1016/j.actbio.2009.05.017.

32. Bat, E.; Kothman, B.H.M.; Higuera, G.A.; van Blitterswijk, C.A.; Feijen, J.; Grijpma, D.W. Ultraviolet light crosslinking of poly(trimethylene carbonate) for elastomeric tissue engineering scaffolds. Biomaterials. 2010, 31, 8696-8705. DOI: 10.1016/j.biomaterials.2010.07.102.

33. Allijn, I.; Ribeiro, M.; Poot, A.A.; Passier, R.; Stamatialis, D. Membranes for Modelling Cardiac Tissue Stiffness In Vitro Based on Poly(trimethylene carbonate) and Poly(ethylene glycol) Polymers. Membranes. 2020, 10, E274. DOI: 10.3390/membranes10100274.

34. Bat, E. Flexible Scaffolds Based on poly(trimethylene carbonate) Networks for Cardiac Tissue Engineering. Ph.D. Thesis, University of Twente, Enschede, The Netherlands, May 2010.

35. Pêgo, A.P.; Vleggeert-Lankamp, C.L.; Deenen, M.; Lakke, E.A.; Grijpma, D.W.; Poot, A.A.; Marani, E.; Feijen, J. Adhesion and growth of human Schwann cells on trimethylene carbonate (co)polymers. J. Biomed. Mater. Res. A. 2003, 67, 876885. DOI: 10.1002/jbm.a.10074.

36. Schüller-Ravoo, S.; Feijen, J.; Grijpma, D.W. Flexible, elastic and tear-resistant networks prepared by photo-crosslinking poly(trimethylene carbonate) macromers. Acta. Biomater. 2012, 8, 3576-3585. DOI: 10.1016/j.actbio.2012.06.004.

37. Pêgo, A.P.; Poot, A.A.; Grijpma, D.W.; Feijen, J. Copolymers of trimethylene carbonate and epsilon-caprolactone for porous nerve guides: Synthesis and properties. Biomater. Sci. Polym. Ed. 2001, 12, 35-53. DOI: 10.1163/156856201744434.

38. Zant, E.; Bosman, M.J.; Grijpma, D.W. Combinatorial synthesis of photocrosslinked biodegradable networks. J. Appl. Biomater. Funct. Mater. 2012, 10, 197-202. DOI: 10.5301/JABFM.2012.10344.

39. Bat, E.; van Kooten, T.G.; Feijen, J.; Grijpma, D.W. Resorbable elastomeric networks prepared by photocrosslinking of high-molecular-weight poly(trimethylene carbonate) with photoinitiators and poly(trimethylene carbonate) macromers as crosslinking aids. Acta. Biomater. 2011, 7, 1939-1948. DOI: 10.1016/j.actbio.2011.01.010.

40. Pêgo, A.P.; Grijpma, D.W.; Feijen, J. Enhanced mechanical properties of 1,3trimethylene carbonate polymers and networks. Polymer. 2003, 44, 6495-6504. DOI: 10.1016/S0032-3861(03)00668-2.

41. Pêgo, A.P.; van Luyn, M.J.A.; Brouwer, L.A.; van Wachem, P.B.; Poot, A.A.; Grijpma, D.W.; Feijen, J. In vivo behavior of poly(1,3-trimethylene carbonate) and copolymers of 1,3-trimethylene carbonate with D,L-lactide or e-caprolactone: Degradation and tissue response. J. Biomed. Mater. Res. A. 2003, 67, 1044-1054. DOI: 10.1002/jbm.a.10121.

42. Pêgo, A.P. Preparation and in vivo Performance of Degradable Two-Ply Nerve Guides Based on (co)Polymers of Trimethylene Carbonate and $\varepsilon$-Caprolactone. Ph.D. Thesis, University of Twente, Enschede, The Netherlands, November 2002. 
43. Pasman, T.; Grijpma, D.W.; Stamatialis, D.; Poot, A.A. Fabricating porous, photocrosslinked poly(trimethylene carbonate) membranes using temperatureinduced phase separation. Polym. Adv. Technol. 2017, 28, 1258-1262. DOI: 10.1002/pat.3956.

44. Zhao, J.; Luo, G.; Wu, J.; Xia, H. Preparation of microporous silicone rubber membrane with tunable pore size via solvent evaporation-induced phase separation. ACS Appl. Mater. Interfaces 2013, 5, 2040-2046. DOI: 10.1021/am302929c.

45. Pervin, R.; Ghosh, P.; Basavaraja, M.G. Tailoring pore distribution in polymer films via evaporation induced phase separation. RSC Adv. 2019, 9, 15593-15605. DOI: 10.1039/c9ra01331h.

46. Srinivasarao, M.; Collings, D.; Philips, A.; Patel, S. Three-dimensionally ordered array of air bubbles in a polymer film. Science. 2001, 292, 79-83. DOI: 10.1126/science.1057887.

47. Dou, Y.; Jin, M.; Zhou, G.; Shui, L. Breath Figure Method for Construction of Honeycomb Films. Membranes 2015, 5, 399-424. DOI: 10.3390/membranes5030399.

48. Wan, L.; Ke, B.; Li, X.; Meng, X.; Zhang, L.; Xu, Z. Honeycomb-patterned films of polystyrene/poly(ethylene glycol): Preparation, surface aggregation and protein adsorption. Sci. China B Chem. 2009, 52, 969-974. DOI: 10.1007/s11426-009-00071.

49. Song, Y.; Wennink, J.W.; Kamphuis, M.M.; Vermes, I.; Poot, A.A.; Feijen, J.; Grijpma, D.W. Effective seeding of smooth muscle cells into tubular poly(trimethylene carbonate) scaffolds for vascular tissue engineering. J. Biomed. Mater. Res. A. 2010, 95, 440-446. DOI: 10.1002/jbm.a.32859.

50. Song, Y.; Wennink, J.W.; Kamphuis, M.M.; Sterk, L.M.; Vermes, I.; Poot, A.A.; Feijen, J.; Grijpma, D.W. Dynamic culturing of smooth muscle cells in tubular poly(trimethylene carbonate) scaffolds for vascular tissue engineering. Tissue Eng. Part A. 2011, 17, 381-387. DOI: 10.1089/ten.TEA.2009.0805.

51. Doytcheva, M.; Dotcheva, D.; Stamenova, R.; Orahovats, A.; Tsvetanov, C.; Leder, J. Ultraviolet-induced crosslinking of solid poly(ethylene oxide). J Appl. Polym. Sci. 1997, 64, 2299-2307. DOI: 10.1002/(SICI)10974628(19970620)64:12<2299::AID-APP5>3.0.CO;2-G.

52. Bat, E.; Plantinga, J.A.; Harmsen, M.C.; van Luyn, M.J.; Zhang, Z.; Grijpma, D.W.; Feijen, J. Trimethylene carbonate and epsilon-caprolactone based (co)polymer networks: Mechanical properties and enzymatic degradation. Biomacromolecules 2008, 9, 3208-3215. DOI: 10.1021/bm8007988.

53. Peponi, L.; Marcos-Fernández, A.; Kenny, J.M. Nanostructured morphology of a random P(DLLA-co-CL) copolymer. Nanoscale Res. Lett. 2012, 7, 103. DOI: 10.1186/1556-276X-7-103. 


\section{Supplementary material of Chapter 5: Development of porous and flexible PTMC membranes for in vitro organ models fabricated by evaporation-induced phase separation}

\section{$1,300 \mathrm{~kg} / \mathrm{mol}$ PTMC}

600 kg/mol PTMC

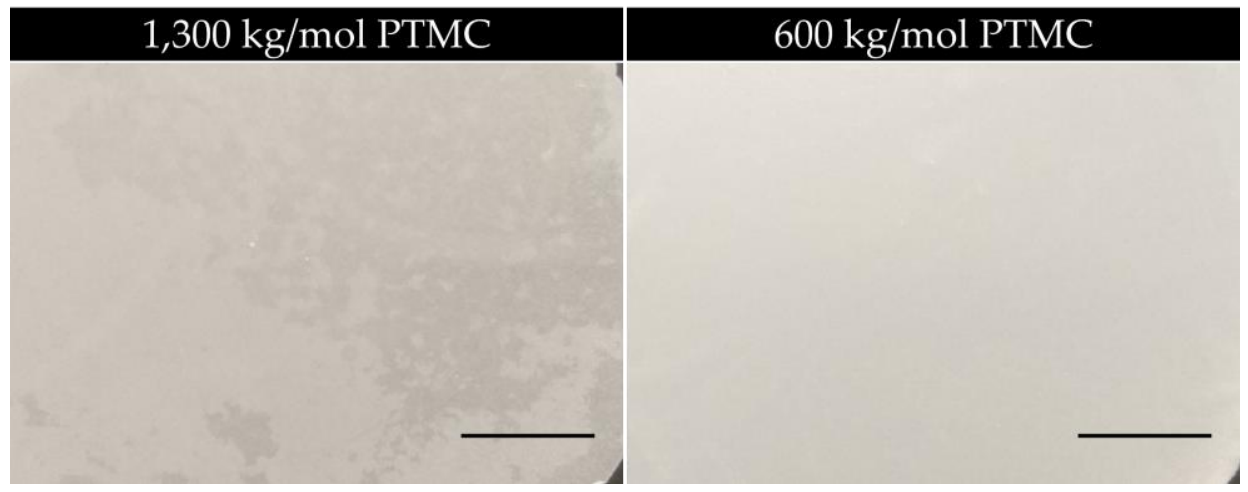

Figure S1. Images of macroscopic morphology of dry PTMC membranes made from PTMC with a MW of $1,300 \mathrm{~kg} / \mathrm{mol}$ or $600 \mathrm{~kg} / \mathrm{mol}$. The ratio of PTMC to hexanol in the polymer dope was 1:1 $(\mathrm{w} / \mathrm{w})$. Scale bar: $1 \mathrm{~cm}$. Membranes made from $1,300 \mathrm{~kg} / \mathrm{mol}$ PTMC were heterogeneous, containing both transparent (dark) and opaque (light) areas indicating differences in porosity, while those made from $600 \mathrm{~kg} / \mathrm{mol}$ PTMC were homogeneous. Images are representative of multiple observations of different membranes. 
- Chapter 5 -

Porous and flexible PTMC membranes fabricated by EIPS

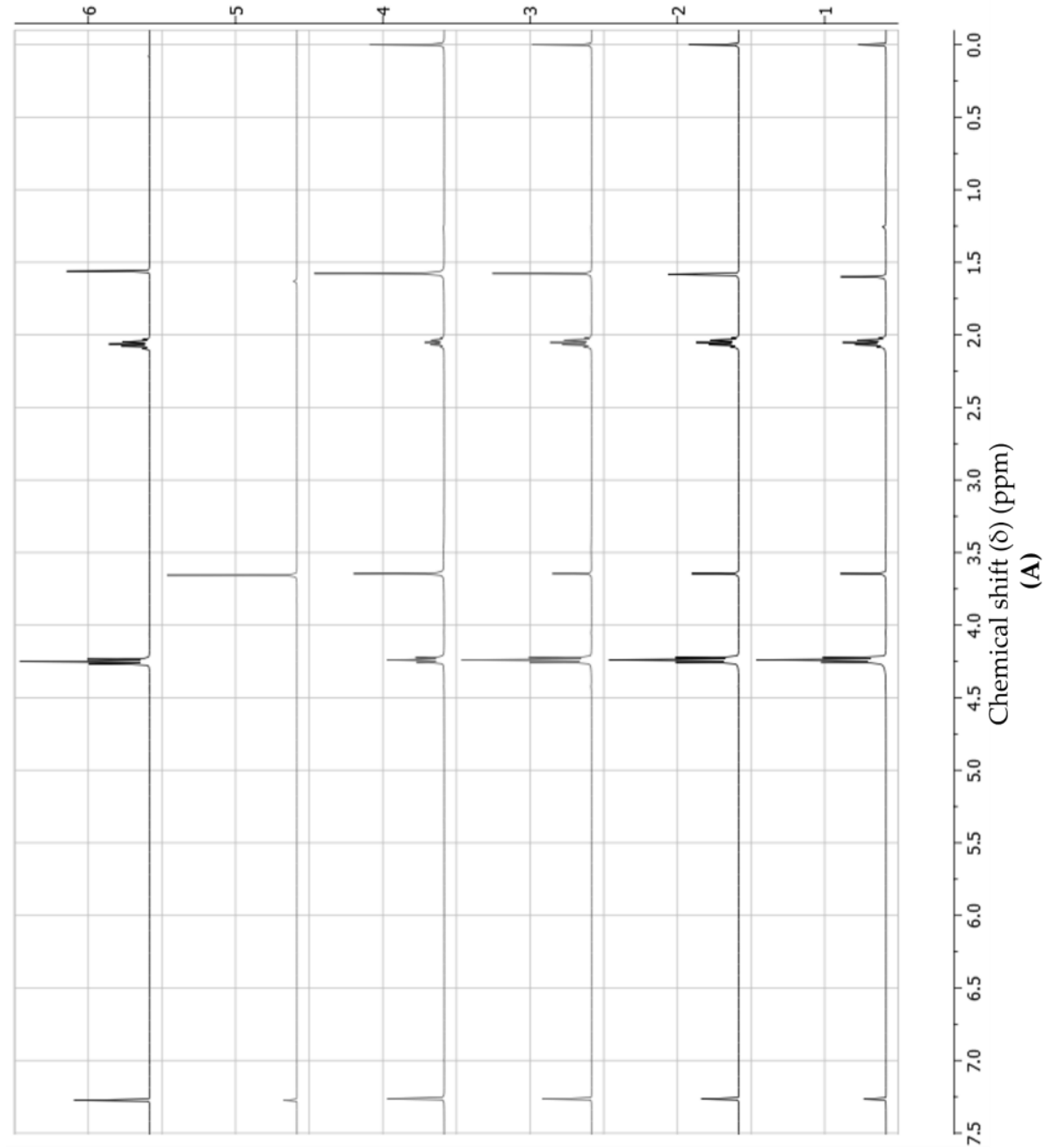

JWLd

Öd

OW

IW

ZW

$\varepsilon W$ 


\section{- Chapter 5 -}

Porous and flexible PTMC membranes fabricated by EIPS
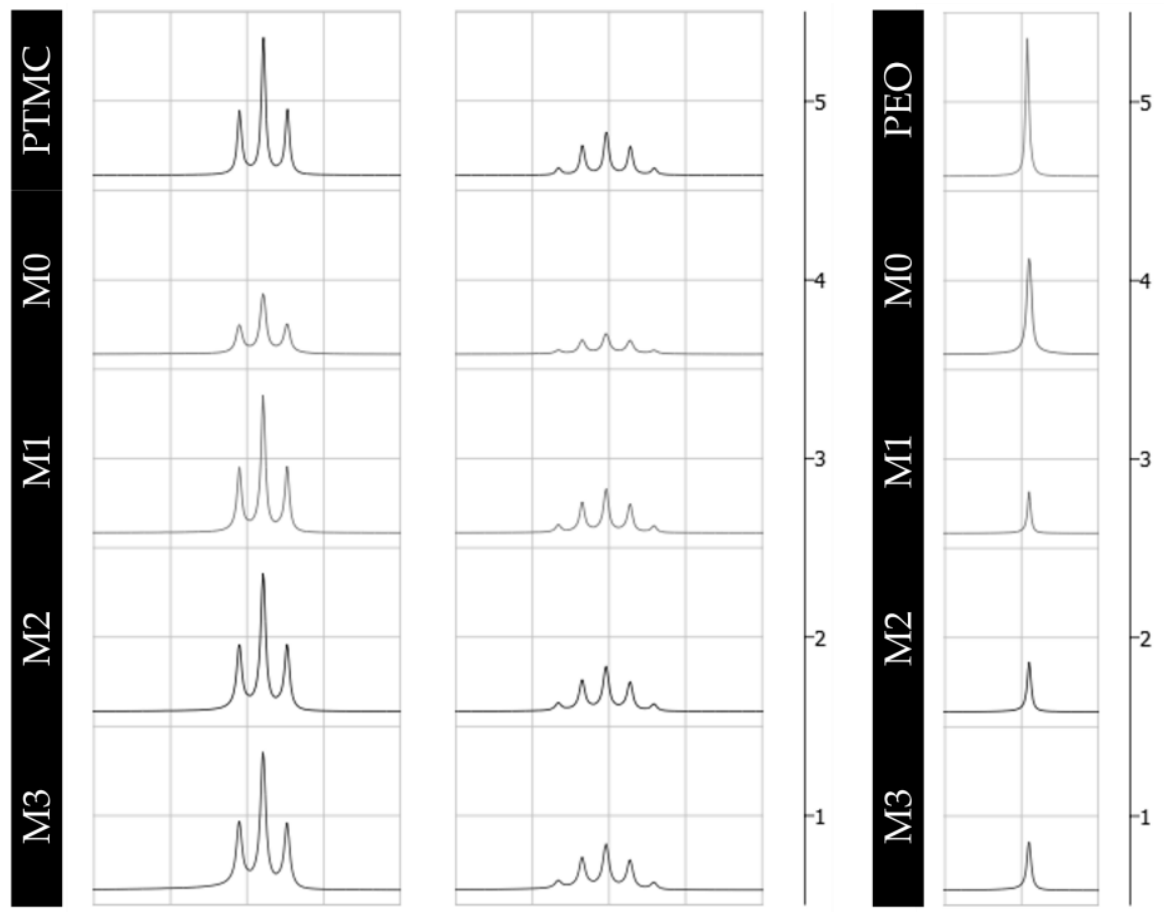

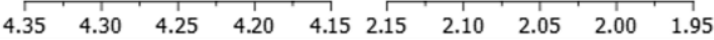
Chemical shift ( $\delta)(\mathrm{ppm})$

(B)

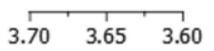

Chemical shift ( $\delta)(\mathrm{ppm})$

(C)

Figure S2. ${ }^{1} \mathrm{H}-\mathrm{NMR}$ spectra of the sol fraction of M0-M3 membranes, pure PTMC and pure PEO. (A) Full spectra of the sol fraction of M0-M3 membranes, PTMC and PEO. (B) Partial spectra of the sol fraction of M0-M3 membranes and PTMC showing characteristic peaks for PTMC. The presence of the triplet around 4.24 ppm and the quintet around $2.06 \mathrm{ppm}$ in the sol fraction of M0-M3 membranes confirms that uncrosslinked PTMC was present in the membranes. (C) Partial spectra of the sol fraction of M0-M3 membranes and PEO showing a characteristic peak for PEO. The presence of the singlet around $3.64 \mathrm{ppm}$ in the sol fraction of M0-M3 membranes confirms that uncrosslinked PEO was present in the membranes. Spectra are representative of multiple samples ( $N \geq 5$ for all membranes and $\mathrm{N}=3$ for the pure components). 
- Chapter 5 -

Porous and flexible PTMC membranes fabricated by EIPS

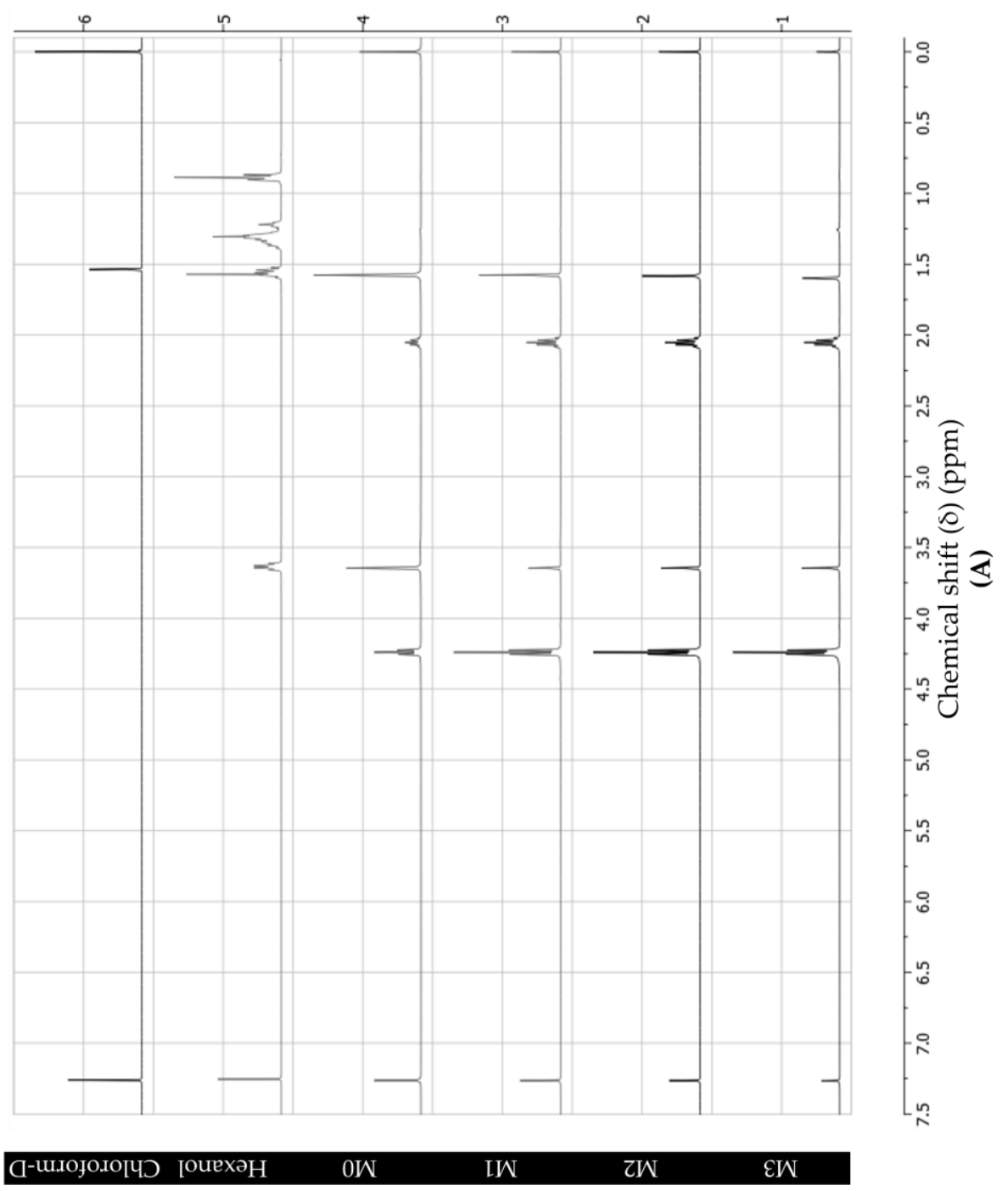


Porous and flexible PTMC membranes fabricated by EIPS

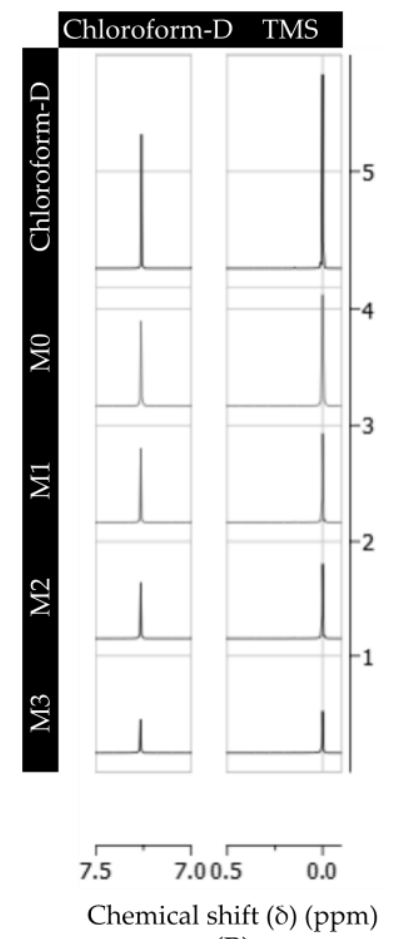

(B)

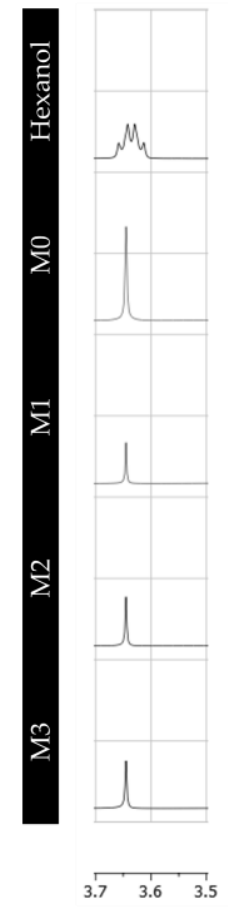

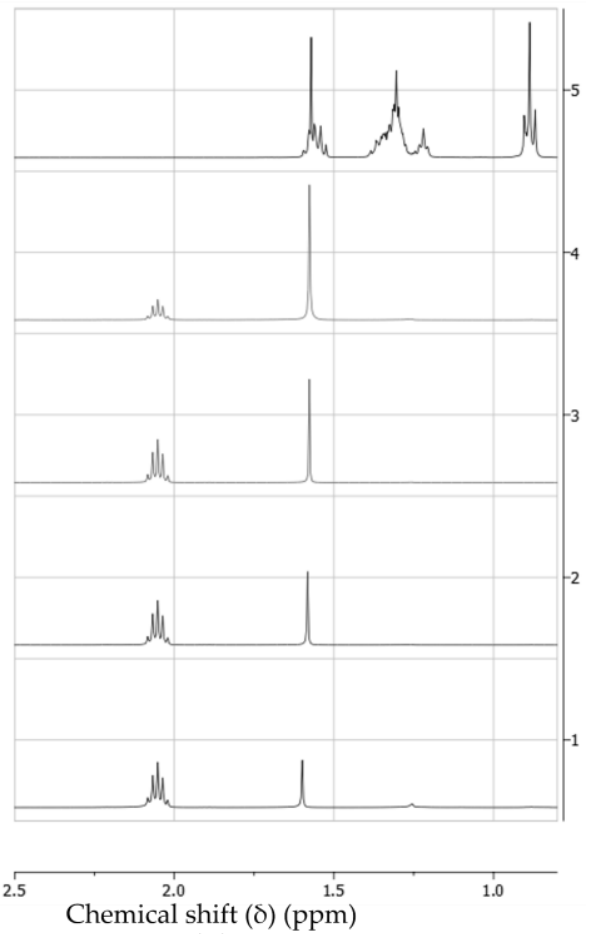

(C)

Figure S3. ${ }^{1} \mathrm{H}-\mathrm{NMR}$ spectra of the sol fraction of M0-M3 membranes, pure chloroform-D and pure hexanol. (A) Full spectra of the sol fraction of M0-M3, chloroform-D and hexanol. (B) Partial spectra of the sol fraction of M0-M3 membranes and chloroform-D showing chloroform and tetramethylsilane (TMS), a reference in the chloroform- $\mathrm{D}$ with a peak at $0.0 \mathrm{ppm}$, in the samples. The ratio between chloroform and TMS did not change, suggesting that M0-M3 membranes contained no chloroform. (C) Partial spectra of the sol fraction of M0M3 membranes and hexanol showing characteristic peaks for hexanol. The absence of a quartet around $3.64 \mathrm{ppm}$ and several multiplets between 1.6 and 0.8 ppm in the sol fraction of M0-M3 membranes confirms that hexanol was not present in the membranes. Spectra are representative of multiple samples $(\mathrm{N} \geq 5$ for all membranes and $\mathrm{N}=3$ for the pure components). 
- Chapter 5 -

Porous and flexible PTMC membranes fabricated by EIPS

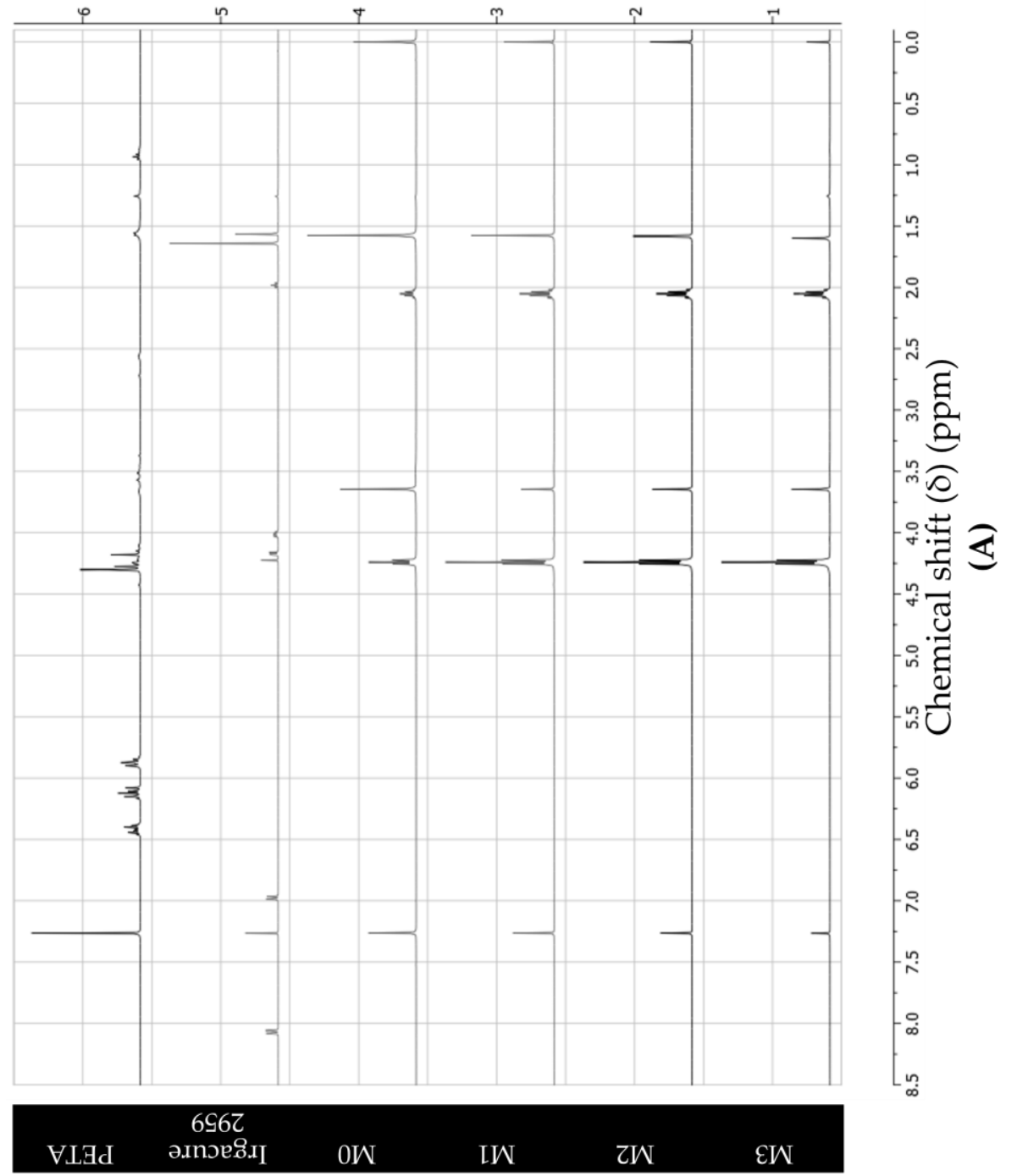


- Chapter 5 -

Porous and flexible PTMC membranes fabricated by EIPS
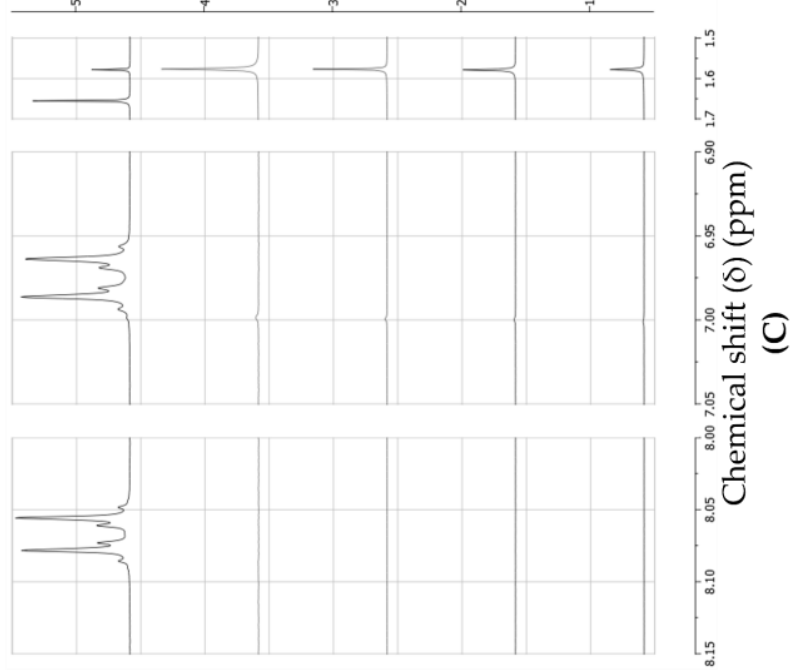

6962
ว.nวe

oW

IW

ZW

$\varepsilon W$
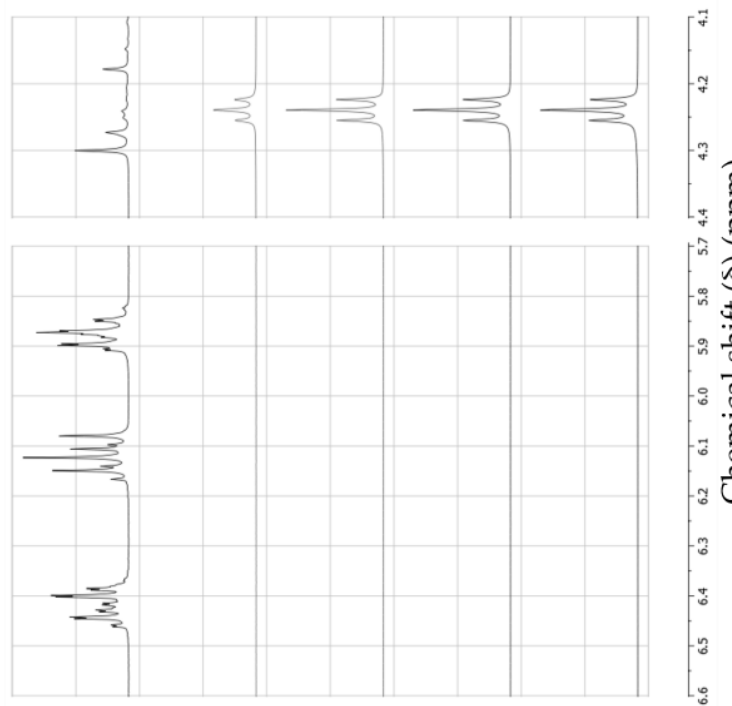

VLAG

OW

IW

ZW

EW

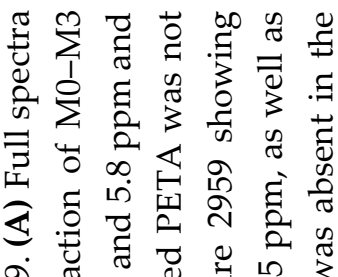

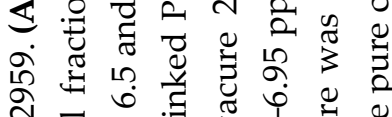

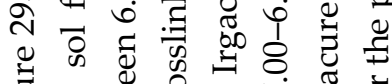

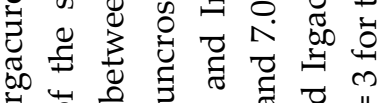

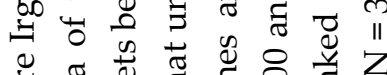

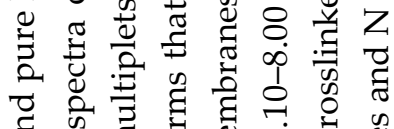

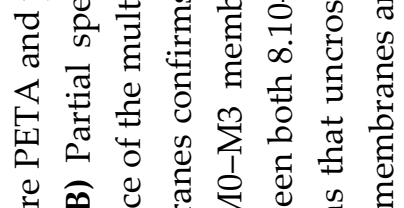

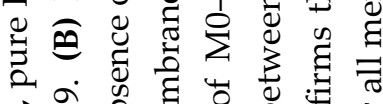

๗ั

ป

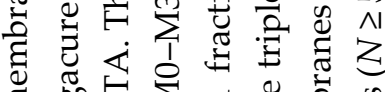

\&

$\sum_{0}^{m}$ च

光

范望

ज.

的 至

光

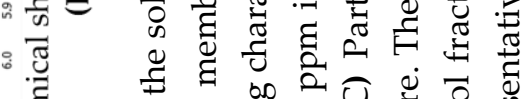

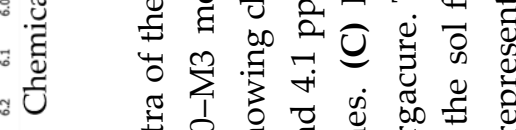

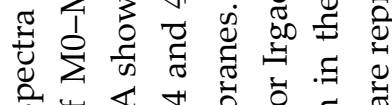

के एँ

茯

正

जं

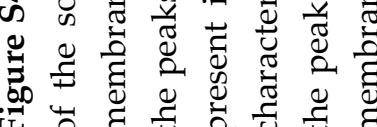




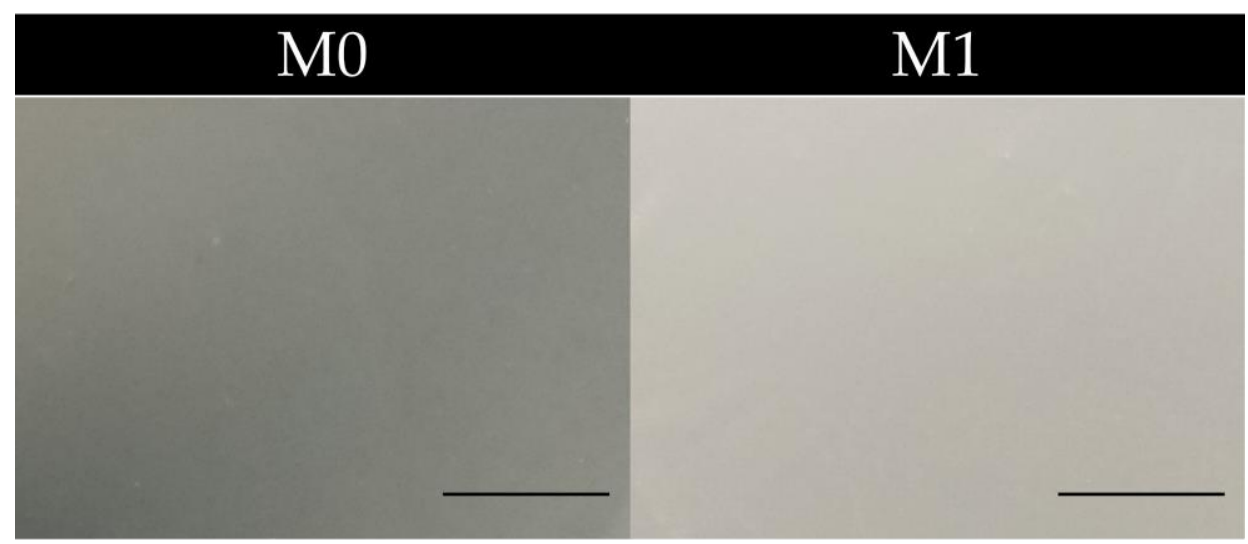

\section{M2 M3}

Figure S5. Images of macroscopic morphology of dry M0-M3 PTMC membranes made from PTMC with a MW of $600 \mathrm{~kg} / \mathrm{mol}$ and different hexanol concentrations in the polymer dope. Scale bar: $1 \mathrm{~cm}$. M0 membranes were transparent, while M1 and M3 membranes were opaque. M0, M1 and M3 membranes were all homogeneous. M2 membranes, however, had a heterogeneous morphology, having both transparent and opaque areas throughout the membranes. Images are representative of multiple observations of different membranes. 
- Chapter 5 -

Porous and flexible PTMC membranes fabricated by EIPS 
- Chapter 5 -

Porous and flexible PTMC membranes fabricated by EIPS 


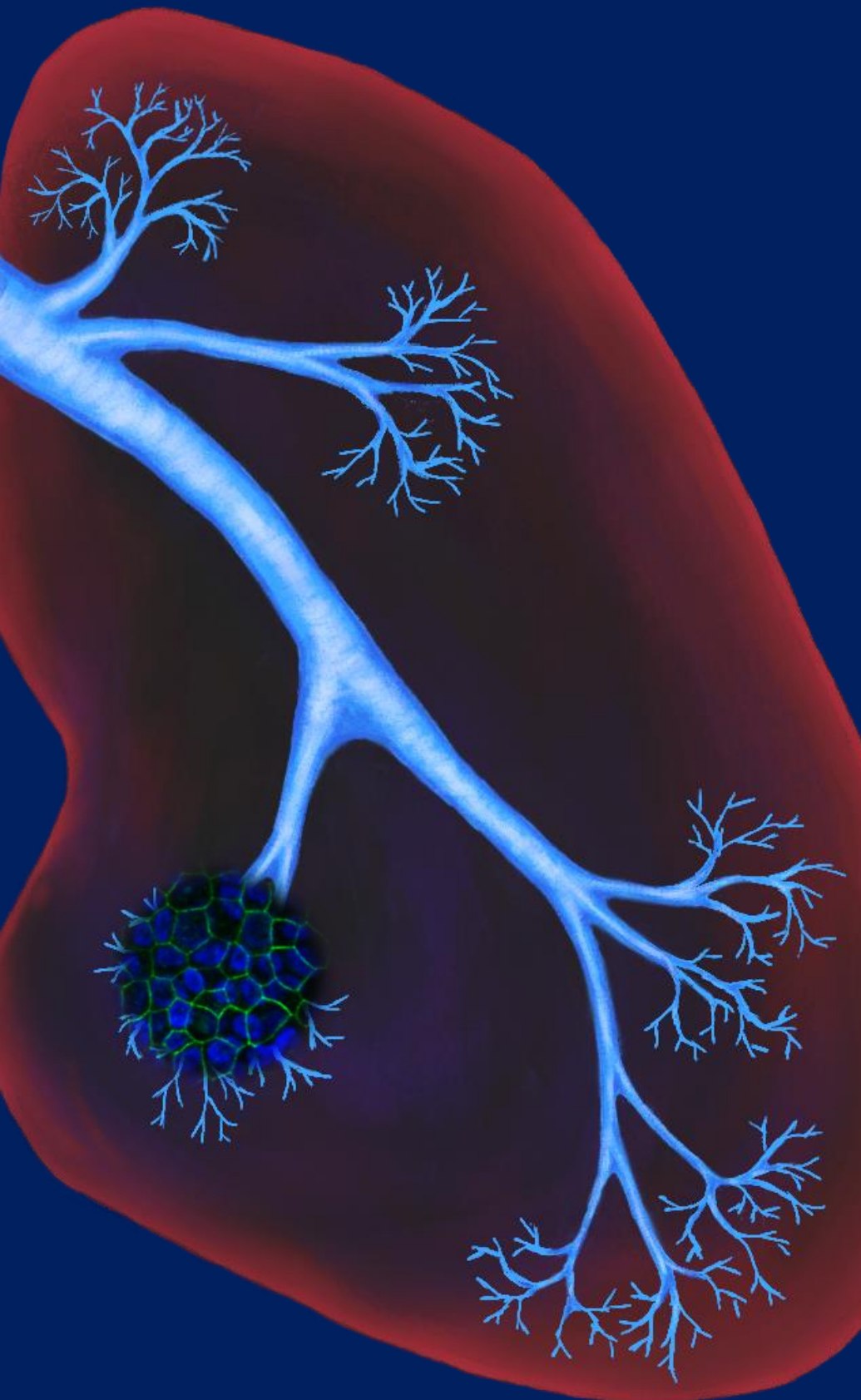




\section{- Chapter 6 -}

\section{Development of an in vitro airway epithelial- endothelial cell culture model on a flexible porous poly(trimethylene carbonate) membrane based on Calu-3 airway epithelial cells and lung microvascular endothelial cells}

Thijs Pasman 1, Danielle Baptista 2, Sander van Riet 3, Roman K. Truckenmüller ${ }^{2}$, Pieter S. Hiemstra ${ }^{3}$, Robbert J. Rottier ${ }^{4}$, Naomi M. Hamelmann ${ }^{5}$, Jos M. J. Paulusse ${ }^{5}$, Dimitrios Stamatialis ${ }^{1}$, André A. Poot 1

1 Technical Medical (TechMed) Centre, Department of Biomaterials Science and Technology, Faculty of Science and Technology, University of Twente, 7522 NB Enschede, The Netherlands

2 Department of Instructive Biomaterials Engineering, MERLN Institute for Technology-Inspired Regenerative Medicine, Maastricht University, 6229 ER Maastricht, The Netherlands

3 Department of Pulmonology, Leiden University Medical Centre, 2300 RC Leiden, The Netherlands

4 Department of Pediatric Surgery and Cell Biology, Erasmus MC-Sophia Children's Hospital, 3000 CB Rotterdam, The Netherlands

5 Department of Biomolecular NanoTechnology, Faculty of Science and Technology, University of Twente, 7522 NB Enschede, The Netherlands

This chapter was adapted from: Pasman, T.; Baptista, D.; van Riet, S.; Truckenmüller, R.K.; Hiemstra, P.S.; Rottier, R.J.; Hamelmann, N.M.; Paulusse, J.; Stamatialis, D.; Poot, A.A. Development of an in vitro airway epithelialendothelial cell culture model on a flexible porous poly(trimethylene carbonate) membrane based on Calu-3 airway epithelial cells and lung microvascular endothelial cells. Membranes. 2021, 11(3), 197. DOI: 10.3390/membranes11030197. 


\section{Abstract}

Due to the continuing high impact of lung diseases on society and the emergence of new respiratory viruses, such as SARS-CoV-2, there is a great need for in vitro lung models that more accurately recapitulate the in vivo situation than current models based on lung epithelial cell cultures on stiff membranes. Therefore, we developed an in vitro airway epithelial-endothelial cell culture model based on Calu-3 human lung epithelial cells and human lung microvascular endothelial cells (LMVECs), cultured on opposite sides of flexible porous poly(trimethylene carbonate) (PTMC) membranes. Calu-3 cells, cultured for two weeks at an air-liquid interface (ALI), showed good expression of the tight junction (TJ) protein Zonula Occludens 1 (ZO-1). LMVECs cultured submerged for three weeks were CD31-positive, but the expression was diffuse and not localised at the cell membrane. Barrier functions of the Calu-3 cell cultures and the co-cultures with LMVECs were good, as determined by electrical resistance measurements and fluorescein isothiocyanate-dextran (FITC-dextran) permeability assays. Importantly, the Calu-3/LMVEC co-cultures showed better cell viability and barrier function than mono-cultures. Moreover, there was no evidence for epithelial- and endothelial-to-mesenchymal transition (EMT and EndoMT, respectively) based on staining for the mesenchymal markers vimentin and $\alpha$-SMA, respectively. These results indicate the potential of this new airway epithelial-endothelial model for lung research. In addition, since the PTMC membrane is flexible, the model can be expanded by introducing cyclic stretch for enabling mechanical stimulation of the cells. Furthermore, the model can form the basis for biomimetic airway epithelial-endothelial and alveolar-endothelial models with primary lung epithelial cells.

Keywords: Calu-3 cells; LMVECs; PTMC; membranes; in vitro lung models; barrier function 


\section{Introduction}

Lung diseases are still among the leading causes of death [1]. Moreover, the emergence of new respiratory viruses with a huge impact on society, such as SARS-CoV-2, further emphasises the relevance of research on lung diseases. Unfortunately, in vitro human lung models frequently do not resemble the in vivo situation, and animal models are increasingly called into question due to ethical issues and generally low predictability for human diseases. Thus, there is an increasing need for the development of in vitro lung models that better mimic human lung tissue.

New in vitro models for airway and alveolar epithelial-endothelial cell interactions are very useful for research of lung diseases. Ideally, primary lung epithelial cells would be used for these models. However, this is often not feasible due to problems concerning the availability and culturing of these cells. For example, although advancements are made to make primary alveolar cells more accessible for research, their availability is limited and maintaining these cells in culture is difficult [2]. Thus, the majority of current lung models still relies on the use of cell lines. The widely used A549 alveolar cell line is less suited for studies on lung barriers since cultures hardly develop a barrier function. Therefore, the Calu-3 cell line, derived from human bronchial adenocarcinoma, has been used extensively in lung barrier models. These cells are regarded as a good model for lung airway epithelia since they can form a pseudostratified cell layer with a proper barrier function at the air-liquid interface (ALI) [3-9]. Moreover, Calu-3 cells are broadly available and easy to maintain. However, they are hardly applied in co-cultures together with endothelial cells, especially microvascular endothelial cells. As a result, many of the current Calu-3 lung barrier models lack the crosstalk between the lung epithelium and endothelium [10,11].

For a proper organ model, the support structure or membrane on which the cells are grown as well as the culture conditions, e.g. mechanical stimulation of the cells, should also be considered as these factors clearly 
affect the cells [12-15]. In some lungs-on-chips (LOCs), cells are cultured on a flexible and elastic membrane, typically made from poly(dimethyl siloxane) (PDMS) [12]. This enables mechanical stimulation of the cells by cyclic stretching or bending of the membrane. However, in many in vitro lung models, including LOCs, the cells are grown on stiff materials such as poly(ethylene terephthalate) (PET) [12], which has a much higher Young's modulus than lung tissue (2-3 GPa [16] vs. $400 \mathrm{~Pa}$ or lower $[17,18])$. Since it is known that cells are affected by the stiffness of the material upon which they are grown [12], membranes made from softer, more flexible materials are preferable to better mimic the lungs.

In this study, we aim to develop a new in vitro airway epithelialendothelial model by co-culturing Calu-3 cells together with human lung microvascular endothelial cells (LMVECs) on flexible porous poly(trimethylene carbonate) (PTMC) membranes. We hypothesise that the lower stiffness of the PTMC membranes compared to PET membranes and the implementation of LMVECs will result in a lung epithelialendothelial barrier model that would be an attractive alternative for existing models. The model could be utilised to study airway diseases that, for example, involve inflammation and damage of the lung, such as chronic obstructive pulmonary disease (COPD), asthma, bronchopulmonary dysplasia (BPD), and cystic fibrosis. Moreover, the effect of external factors (e.g. pollutants, pathogens, and medicines) on barrier function could be examined. Furthermore, the model could form the first step in developing biomimetic airway epithelial-endothelial and alveolar-endothelial models with primary lung epithelial cells. The latter would enable studies on alveolar diseases such as acute respiratory distress syndrome (ARDS) and study of the emphysema component of COPD. We chose the Calu-3 cells because of their robustness and proper barrier function of cell layers at ALI. The PTMC membranes have a Young's modulus around $10 \mathrm{MPa}$, which is much lower than that of PET membranes, thus better mimicking the Young's modulus of lung tissue. PTMC has been successfully used to fabricate films, membranes, and scaffolds for tissue engineering [19-21]. Moreover, PTMC-based films and 
scaffolds with suitable mechanical properties for cyclic stretch have been fabricated [20,22]. Here, we used PTMC membranes with various porosities, pore sizes, and water transport properties, which were developed earlier [23]. The PTMC membranes were either coated with levodopa (L-DOPA), a mixture of fibronectin, collagen I, and bovine serum albumin (FN/Col I/BSA) or L-DOPA followed by FN/Col I/BSA. LDOPA is a precursor of dopamine and acts as a bioadhesive [24] that can stimulate the adsorption of proteins [25-27]. Chevtchik et al. have used LDOPA as a bioadhesive to achieve optimal collagen IV coating of polymeric membranes for culturing kidney epithelial cells [26]. FN/Col I/BSA protein coatings have been successfully used as a coating for the culturing of primary lung epithelial cells [28]. The combination of the PTMC membrane and coating that resulted in the best adhesion and proliferation of Calu-3 cells was subsequently used to develop a coculture of Calu-3 cells and LMVECs with the former cells cultured at ALI. Co- and mono-cultures were stained for Zonula Occludens 1 (ZO-1), a protein associated with tight junctions (TJs) and CD31, a marker for endothelial cells, and characterised in terms of cell viability and barrier function. Furthermore, the cultures were stained for mesenchymal markers to investigate epithelial- and endothelial-to-mesenchymal transition (EMT and EndoMT, respectively) and expression of these markers was compared to that of human vascular smooth muscle cells (SMCs). Results were compared to data from co- and mono-cultures on commercial PET membranes and discussed in view of the literature. 


\section{Materials and methods}

\subsection{Materials}

Acetic acid (cat. W200611), BSA (cat. A7030), calcein-AM (cat. 56496), Corning ${ }^{\circledR}$ Transwell ${ }^{\circledR}$ PET membrane cell culture inserts (cat. CLS3460), ethidium homodimer I (cat. E1903), fluorescein isothiocyanate-dextran (FITC-dextran) (cat. 46944), gelatin (cat. G1393), formaldehyde (FA) (cat. F1635), L-DOPA (cat. D9628), tris(hydroxymethyl) aminomethane (cat. 25,285-9), Triton ${ }^{\mathrm{TM}}$ X-100 (cat. X100), and Tween ${ }^{\circledR 20}$ (cat. P9416) were all purchased from Sigma-Aldrich, St. Louis, MO, USA.

Alexa fluor Donkey $\alpha$ Rabbit 568 antibody (cat. A10042), Alexa fluor Goat $\alpha$ Mouse 488 antibody (cat. A11029), and DAPI FluoroPure ${ }^{\mathrm{TM}}$ (cat. D21490) were acquired from Life Technologies Corporation, Eugene, OR, USA.

$\alpha$-SMA antibody (cat. MA5-11547), CD31 antibody (cat. PA5-95186), vimentin antibody (cat. MA5-14564), and ZO-1 antibody (cat. 33-9100) were bought from Invitrogen, Rockford, IL, USA.

DMEM (Dulbecco's Modified Eagle's Medium) (cat. 12491-015), fetal bovine serum (FBS) (cat. 10500-064), Glutamax ${ }^{\mathrm{TM}}$ 100X (cat. 35050-061), and penicillin-streptomycin (P/S) (cat. 15140-122) were obtained from Gibco, Grand Island, NJ, USA.

Dulbecco's PBS without $\mathrm{Ca}^{2+} / \mathrm{Mg}^{2+}$ (DPBS) (cat. 14190-094) and 0.25\% trypsin/EDTA (cat. 25200-072) were purchased from Gibco, Paisley, UK.

Furthermore, we ordered Alexa Fluor ${ }^{\mathrm{TM}} 568$ Phalloidin (Thermo Fisher Scientific, Waltham, MA, USA, cat. A12380), collagen I (Purecol) (Advanced Biomatrix, Carlsbad, CA, USA, cat. 5005), Dako fluorescent mounting medium (Dako North America, Carpinteria, CA, USA, cat. S3023), Eagle's Minimum Essential Medium (EMEM) (Lonza Group Ltd., Basel, Switzerland, cat. BE12-662F), Falcon ${ }^{\circledR}$ 12-well tissue culture-treated polystyrene (TCPS) permeable support companion plates (Corning $\mathrm{GmbH}$, Wiesbaden, Germany, cat. 353503), fibronectin (EMD Millipore Corporation, Billerica, MA, USA, cat. FC010), Hoechst (Thermo Fisher Scientific, Waltham, MA, USA, cat. H1399), microvascular endothelial cell 
growth medium kit enhanced (Pelobiotech $\mathrm{GmbH}$, Planegg/Martinsried, Germany, cat. PB-MH-100-4099), Nunc ${ }^{\mathrm{TM}}$ MicroWell $^{\mathrm{TM}}$ 96-well, Nunclon Delta-treated, flat-bottom microplates (Thermo Fisher Scientific, Roskilde, Denmark, cat. 237107), and transforming growth factor $\beta$ (TGF$\beta)$ (PeproTech Inc., Cranbury, NJ, USA, cat. 100-21).

\subsection{Membranes and coatings}

\subsubsection{PTMC membrane fabrication}

The synthesis of PTMC and fabrication of the membranes was described in detail previously [23]. Therefore, here we describe those only briefly. PTMC synthesis: PTMC was synthesised by ring-opening polymerisation of trimethylene carbonate (TMC). After synthesis, the PTMC was purified and dried. Polymer dope composition: Polymer dopes for membrane fabrication contained PTMC of $600 \mathrm{~kg} / \mathrm{mol}$, poly(ethylene oxide) (PEO), pentaerythritol triacrylate (PETA), Irgacure 2959, hexanol, and chloroform. Hexanol was added as a non-solvent in a 1:0, 1:1, and 1:3 weight ratio of PTMC to hexanol. Membrane fabrication: Polymer dopes were cast on silicon wafers, and evaporation-induced phase separation (EIPS) was used to form the membranes, which are referred to as M0, M1, and $\mathrm{M} 3$ according to the weight ratio of PTMC to hexanol (i.e. 1:0, 1:1, and 1:3, respectively). All membranes were photo-crosslinked with UV-light, washed, and dried.

\subsubsection{Mounting of membranes in inserts}

PTMC membranes in cell culture inserts: Circular samples with a diameter of $15 \mathrm{~mm}$ were punched from PTMC membranes. PET membranes of cell culture inserts were removed. The PTMC membrane samples were then secured to the underside of the inserts (substrate side [23] facing up) using poly(ether ether ketone) (PEEK) membrane rings (Figure S1 in Supplementary Material), provided by Ronald C. van Gaal (Department of Biomedical Engineering, Biomedical Materials and Biochemistry, Eindhoven University of Technology, Eindhoven, the Netherlands). Plate rings (Figure S1), also provided by Ronald C. van Gaal, were placed on top of wells of Falcon 12-well companion plates to 
elevate the inserts to allow cell culture medium to reach the underside of the inserts. The surface area of the PTMC membranes in the assembled inserts was the same as that of the inserts with the PET membranes (i.e. $1.12 \mathrm{~cm}^{2}$ ). Membrane and insert sterilisation: Assembled inserts with PTMC membranes and inserts with PET membranes were kept in $70 \%$ ethanol for at least one hour. Afterwards, they were dried, washed, and left in culture plates in DPBS in a cell culture incubator.

\subsubsection{Membrane coating}

After sterilisation, membrane samples either received no coating, an FN/Col I/BSA coating, an L-DOPA coating, or an L-DOPA coating followed by an FN/Col I/BSA coating. L-DOPA coating: The L-DOPA coating was performed following the protocol of Chevtchik et al. [26]. A $10 \mathrm{mmol} / \mathrm{L}$ Tris-buffer in MilliQ water was made. Acetic acid was added to lower the $\mathrm{pH}$ to 8.5 . The buffer was sterilised by filtration through a 0.2 $\mu \mathrm{m}$ pore filter. Membrane samples in inserts were incubated in Tris-buffer for at least one hour. For the L-DOPA solution, a Tris-buffer with $2 \mathrm{mg} / \mathrm{mL}$ L-DOPA was sealed and left to stir for $45 \mathrm{~min}$. at $37^{\circ} \mathrm{C}$. Subsequently, the solution was immediately filtered through a $0.2 \mu \mathrm{m}$ pore filter and added on top and underneath the membrane samples. Samples were then kept at $37^{\circ} \mathrm{C}$ in an incubator for $20 \mathrm{~h}$. Afterwards, the L-DOPA solution had turned almost completely black. Finally, samples were washed four times with DPBS and kept in DPBS at $37^{\circ} \mathrm{C}$ in a cell culture incubator. While M1 and M3 PTMC membranes were white opaque before the L-DOPA coating, they were dark grey opaque afterwards, confirming that the LDOPA coating was present on the membranes. The L-DOPA coating was also noticeable on M0 and PET membranes and TCPS, which all remained transparent. FN/Col I/BSA coating: A mixture consisting of fibronectin (5 $\mu \mathrm{g} / \mathrm{mL})$, collagen I (30 $\mu \mathrm{g} / \mathrm{mL})$, and BSA $(10 \mu \mathrm{g} / \mathrm{mL})$ in DPBS was sterilefiltered through a $0.2 \mu \mathrm{m}$ pore filter and added to samples (both well and insert), which were then incubated for one hour at $37^{\circ} \mathrm{C}$. Afterwards, they were washed once with DPBS and kept at $37^{\circ} \mathrm{C}$ in a cell culture incubator. 


\subsection{Cell culture}

\subsubsection{Cell culture media}

Calu-3 medium: Cell culture medium used for the Calu-3 cells consisted of EMEM (88 vol\%), FBS (10 vol\%), P/S (1 vol\%), and Glutamax ${ }^{\mathrm{TM}}$ (1 vol\%). LMVEC medium: A 'microvascular endothelial cell growth medium kit enhanced' was used for the culturing of LMVECs, according to the manufacturer's instructions.

Co-culture medium: Cell culture medium for the co-cultures consisted of Calu-3 (50 vol\%) and LMVEC medium (50 vol\%), as described above.

SMC medium: Culture medium for the SMCs consisted of DMEM (88 vol\%), FBS (10 vol\%), P/S (1 vol\%), and Glutamax ${ }^{\mathrm{TM}}$ (1 vol\%). For serum deprivation and stimulation of the SMCs with TGF- $\beta$, culture media were made consisting of DMEM (97.6 vol\%), FBS (0.4 vol\%), P/S (1 vol\%), and Glutamax $^{\mathrm{TM}}(1 \mathrm{vol} \%)$, without and with TGF- $\beta(5 \mathrm{ng} / \mathrm{mL})$, respectively.

\subsubsection{Cells and general cell culturing}

Calu-3 cells: Calu-3 cells (passage 8 ) were kindly provided by the department of Instructive Biomaterials Engineering, Maastricht University, Maastricht, The Netherlands. The cells were cultured in culture flasks with Calu-3 medium. At 70\% confluence, cells were trypsinised and split before using them in plates and inserts between passage 11 and 16.

LMVECs: LMVECs were bought from Pelobiotech $\mathrm{GmbH}$, Planegg/Martinsried, Germany, cat. ACBRI 468. Cells were cultured in LMVEC medium in culture flasks coated with $0.1 \%$ gelatin. After reaching confluence, cells were trypsinised and split once before using them on inserts at passage 8 .

SMCs: SMCs from umbilical veins (passage 3) were received from Medical Spectrum Twente hospital, Enschede, The Netherlands, and cultured in SMC medium in culture flasks coated with $0.1 \%$ gelatin. At $70 \%$ confluence, cells were trypsinised and split before using them on inserts at passage 7 . All cells were grown in a humid environment at 37 ${ }^{\circ} \mathrm{C}$ with $5 \% \mathrm{CO}_{2}$. Cell culture medium was refreshed three times a week for all cells. 


\subsubsection{Cell seeding and culturing on inserts}

Calu-3 cell culturing on PTMC membranes for membrane coating assessment: Calu-3 cells (passage 11) were seeded in cell culture inserts on M0, M1, M3, and PET membranes or in TCPS well plates with different coatings at a cell density of 120,000 cells $/ \mathrm{cm}^{2}$. Cells were left to grow submerged for 8 days, after which those in the inserts were cultured at ALI by removing the Calu-3 medium from the insert. The Calu-3 medium was refreshed three times a week throughout the experiment. Calu-3 cells were also quickly washed three times a week with DPBS during ALI culturing to remove cell debris and mucus. After 2 weeks at ALI, the cells were fixed with $4 \%$ paraformaldehyde (PFA) and stained with DAPI and phalloidin (see Sections 2.3.7 and 2.3.8). The cells on TCPS were cultured submerged for 23 days, and subsequently fixed and stained in the same manner.

Cell culturing on M3 and PET membranes for ZO-1/CD31 staining and live/dead staining, and barrier function assays: LMVECs (passage 8) were seeded on the underside of cell culture inserts on L-DOPA+FN/Col I/BSAcoated M3 and PET membranes at a density of 80,000 cells/ $\mathrm{cm}^{2}$ by flipping the cell culture inserts upside down. Cells were kept moist during attachment (approximately $2 \mathrm{~h}$ ), after which the inserts were placed in 12 well plates in the normal orientation, and LMVEC medium was added to both the inserts and wells. The next day (day 1, see Scheme 1A), Calu-3 cells were seeded on the topside of selected membranes at 120,000 cells $/ \mathrm{cm}^{2}$ in Calu-3 medium. For these co-cultures, cells were kept in their respective media. The next day (day 2), co-cultures were switched to coculture medium, while mono-cultures were kept in their respective media. Samples with Calu-3 cells were transferred to ALI on day 9. Three times a week, the medium was refreshed, and Calu-3 cells were quickly washed with DPBS. Electrical resistance and FITC-dextran permeability assays were performed on day 22 and 23, respectively (13 and 14 days at ALI, respectively) (see Sections 2.3.4 and 2.3.5). On day 24, the cells were fixed with $4 \%$ PFA or subjected to live/dead staining (see Sections 2.3.7 and 2.3.8). 


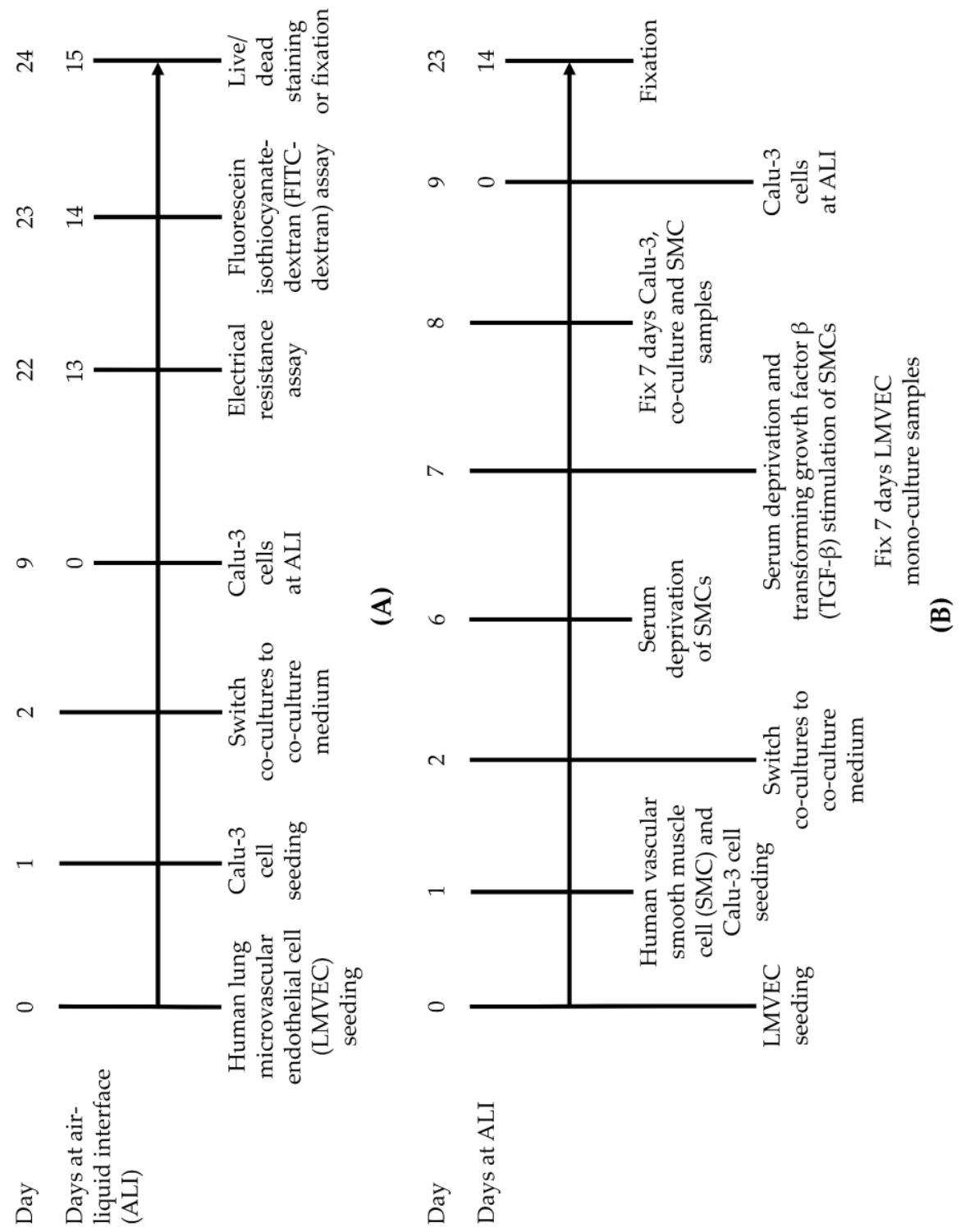


Scheme 1. Timelines of cell culture experiments on poly(trimethylene carbonate) (PTMC) M3 and poly(ethylene terephthalate) (PET) membranes. Timelines were similar for co- and mono-cultures. (A) Timeline of cell culture experiments for tight junction protein Zonula Occludens 1 (ZO-1) and endothelial marker CD31 staining, live/dead staining, and barrier function assays. (B) Timeline of cell culture experiments for expression of epithelial-to-mesenchymal (EMT)/endothelial-to-mesenchymal (EndoMT) markers.

Cell culturing on M3 and PET membranes for EMT and EndoMT marker staining: For the expression of EMT/EndoMT markers, Calu-3 cells (passage 11) and LMVECs (passage 8) were seeded in the same manner as for the assessment of ZO-1/CD31 staining (see above) on LDOPA+FN/Col I/BSA-coated M3 and PET membranes (Scheme 1B). Moreover, on day 1 , SMCs (passage 7) were seeded at 10,000 cells $/ \mathrm{cm}^{2}$ on L-DOPA+FN/Col I/BSA-coated M3 and PET membranes as positive controls for mesenchymal marker expression. SMCs were cultured in SMC medium. Calu-3/LMVEC co-cultures were switched to co-culture medium on day 2. On day 6, SMCs were serum-deprived by receiving SMC medium with 0.4 vol\% FBS. On day 7 , the SMCs were stimulated with $5 \mathrm{ng} / \mathrm{mL}$ TGF- $\beta$. Moreover, LMVEC mono-culture samples were fixed. On day 8, samples of Calu-3 mono-cultures, co-cultures, and all samples of the SMC cultures were fixed. At day 9, the remaining samples containing Calu-3 cells were transferred to ALI, as mentioned before. On day 23, all remaining Calu-3, LMVEC, and co-culture samples were fixed with 4\% PFA (Section 2.3.7). Cell culture medium was refreshed three times a week, and during ALI culturing, the Calu-3 cells were quickly washed with DPBS three times a week as well.

\subsubsection{Electrical resistance measurements}

12-well Transwell ${ }^{\circledR}$ inserts with L-DOPA+FN/Col I/BSA-coated PET or M3 PTMC membranes were placed in a cellZscope system (nanoAnalytics $\mathrm{GmbH}$, Münster, Germany). Fresh cell culture medium was added to the inserts. Preliminary measurements with a duration of three hours showed that the electrical resistance was stable during that time (data not shown). Nevertheless, measurement time in the case of membranes with cells was 
as short as possible $(<1 \mathrm{~h})$ to limit any potential influence of the assay on the cells. Electrical resistance data were collected by cellZscope software version 2.2.1 from nanoAnalytics (Münster, Germany). Data of two separate experiments were gathered, each experiment including samples of different membranes of each membrane type (total $\mathrm{N} \geq 3$ for membranes without cells, $\mathrm{N} \geq 7$ for samples with cells). For the type of reference insert, the option 'Corning 12-well transparent $\left(1.12 \mathrm{~cm}^{2}\right)$ ' was chosen in the cellZscope software. The electrical resistance was measured from $1 \mathrm{~Hz}$ to $100 \mathrm{kHz}$.

\subsubsection{FITC-dextran permeability assay}

Sterile solutions of $1 \mathrm{mg} / \mathrm{mL}$ FITC-dextran $(4 \mathrm{~kg} / \mathrm{mol})$ in cell culture medium were made. Membrane samples with or without cells were washed once with medium. Subsequently, $1.2 \mathrm{~mL}$ fresh medium was added underneath the inserts and $0.6 \mathrm{~mL}$ FITC-dextran solution was added to the inserts, on top of the membranes, for $60 \mathrm{~min}$. at $37^{\circ} \mathrm{C}$. Afterwards, samples of the medium underneath the inserts were taken in triplicate for analysis. The membrane samples with cells were then washed with medium and received fresh medium. Analysis was done by transferring the medium samples to black 96-well Microplates. Pure medium and a concentration series of FITC-dextran solutions were used as reference samples in each plate. The fluorescence was measured the same day by placing the plates in a Tecan Infinite M200 Pro® plate reader with the Tecan i-control ${ }^{\mathrm{TM}}$ software. The excitation and emission wavelengths were $490 \mathrm{~nm}$ and $520 \mathrm{~nm}$, respectively. Data were collected from two separate experiments, each using samples of different membranes of each membrane type (total $\mathrm{N}=4$ for membranes without cells, $\mathrm{N} \geq 9$ for samples with cells). The data were corrected for the fluorescence values of the pure medium. A calibration curve was made to translate the fluorescence data to FITC-dextran concentrations. The apparent permeability coefficient ( $\mathrm{P}_{\mathrm{app}}$ ) in $\mathrm{cm} / \mathrm{s}$ of FITC-dextran diffusion across the membrane and cell layers was then calculated using Equation (1) in which dQ is the accumulated FITC-dextran in mg in the acceptor compartment (i.e. the well), $\mathrm{dt}$ is the duration of the assay in seconds, $\mathrm{C}_{0}$ 
is the initial concentration of FITC-dextran in the donor compartment (the insert) in $\mathrm{mg} / \mathrm{cm}^{3}$, and $\mathrm{A}$ is the surface area of the membrane, i.e. $1.12 \mathrm{~cm}^{2}$ for both the M3 and PET membranes.

$$
\mathrm{P}_{\mathrm{app}}=\frac{\mathrm{dQ} / \mathrm{dt}}{\mathrm{C}_{0} \cdot \mathrm{A}}
$$

\subsubsection{Statistical analysis}

For statistical analysis of the electrical resistance and $\mathrm{Papp}_{\text {apdata }}$ a one-way ANOVA was performed using a Bonferroni post-hoc test with $\mathrm{P}<0.05$ in the Graphpad Prism 5 software (San Diego, CA, USA).

\subsubsection{Cell fixation}

Cells were quickly washed once with $37^{\circ} \mathrm{C}$ DPBS, immediately followed by incubation in sterile $4 \mathrm{vol} \%$ PFA of $37^{\circ} \mathrm{C}$, both on top and underneath the inserts, for $45 \mathrm{~min}$. at $20^{\circ} \mathrm{C}$. Cells were washed three times with DPBS, left in DPBS, and stored at $4{ }^{\circ} \mathrm{C}$.

\subsubsection{Immunocytochemistry}

Calu-3 cell culturing, ZO-1/CD31 staining, and EMT and EndoMT markers: Fixed membrane samples with cells were incubated with a 0.1 vol\% Triton ${ }^{\mathrm{TM}} \mathrm{X}-100$ solution in DPBS for $10 \mathrm{~min}$. at $20^{\circ} \mathrm{C}$ to permeabilise the cells. Subsequently, the samples were kept in a blocking solution, consisting of $5 \mathrm{wt} \%$ BSA, 2 vol\% FBS, and 0.1 vol\% Tween ${ }^{\circ} 20$ in DPBS, for at least $60 \mathrm{~min}$. Calu-3 samples for the assessment of the polymer membrane coating were incubated with a phalloidin solution (1:200 dilution in DPBS) for $30 \mathrm{~min}$. at $20^{\circ} \mathrm{C}$ to stain F-actin. Afterwards, these samples were incubated with a DAPI solution (see below). All other samples were incubated overnight at $4{ }^{\circ} \mathrm{C}$ with either a ZO- 1 or $\alpha$-SMA antibody (1:300 and 1:200 dilution in DPBS, respectively). The next day, samples were incubated with an Alexa fluor Goat $\alpha$ Mouse 488 solution (1:200 dilution in DPBS) for $2 \mathrm{~h}$ at $20{ }^{\circ} \mathrm{C}$. Subsequently, samples were incubated overnight at $4{ }^{\circ} \mathrm{C}$ with either a CD31 or vimentin antibody (both 1:500 dilution in DPBS). The next day, samples were incubated with an Alexa fluor Donkey $\alpha$ Rabbit 568 antibody (1:500 dilution in DPBS) for 2 
$\mathrm{h}$ at $20^{\circ} \mathrm{C}$. Samples were then incubated with a DAPI solution $(300 \mathrm{nM}$ in DPBS) for $20 \mathrm{~min}$. at $20^{\circ} \mathrm{C}$. All incubation steps were done in the dark, and all samples were washed at least 2 times with DPBS between all steps. Afterwards, Calu-3 samples were mounted on microscopy slides with mounting medium and then imaged.

Calu-3 samples for the assessment of the polymer membrane coating were imaged with an EVOS FL AMF4300 microscope (Advanced Microscopy Group, Bothell, WA, USA), whereas images of the cellular ZO-1/CD31 staining and EMT/EndoMT markers were made with a Leica TCS SP5 confocal microscope with the Leica Application Suite software (Leica Microsystems Inc., Buffalo Grove, IL, USA). The Calu-3 samples for assessment of the membrane coating were taken from one experiment $(\mathrm{N}$ $=1$ ), and samples for the ZO-1/CD31 staining originated from different membranes of two separate experiments (total $\mathrm{N}=3$ for each of the stainings). Samples for the staining of EMT and EndoMT markers came from different membranes of one experiment $(\mathrm{N}=3)$.

Live/dead staining for cell viability: A solution containing $2 \mu \mathrm{M}$ calcein, 4 $\mu \mathrm{M}$ ethidium homodimer I, and $16 \mu \mathrm{M}$ Hoechst in cell culture medium was prepared. The unfixed cells were sensitive to the staining procedure, especially to washing with DPBS, which affected their morphology or even adhesion. Therefore, samples were washed once with their respective culture medium instead of DPBS. Cells received the staining solution and were incubated for $20 \mathrm{~min}$, after which the samples were washed with medium, and the membranes were placed on microscopy slides. Samples were immediately imaged on a Nikon Eclipse 400 microscope (Nikon, Tokyo, Japan) with the Nikon NIS-Elements Imaging software (version 4.51.01). Samples were taken from different membranes of two separate experiments (total $\mathrm{N}=3$ ). 


\section{Results and discussion}

\subsection{Calu-3 cell attachment on PTMC membranes with L-DOPA and FN/Col I/BSA coating}

Adhesion of Calu-3 cells on PTMC M0, M1, and M3 membranes was assessed by staining their nuclei (Figure 1). The PTMC membranes had diverse properties, as described in a previous paper [23], most notably concerning their porosity and water transport. Briefly, M0 membranes were non-porous and non-permeable. M1 and M3 membranes had porosities of $21 \%$ and $42 \%$, and water permeances of 17,000 and 41,000 $\mathrm{L} /\left(\mathrm{m}^{2} \cdot \mathrm{h} \cdot \mathrm{bar}\right)$, respectively. Furthermore, pore sizes of M1 and M3 membranes were 5-8 and 7-8 $\mu \mathrm{m}$, respectively. Different coatings were applied on these PTMC membranes, as well as on PET membranes with $0.4 \mu \mathrm{m}$ pores and on TCPS cell culture plates. Calu- 3 cells were stained after eight days of submerged culture and two weeks of culture at ALI on PTMC and PET membranes, and after 23 days of submerged culture on TCPS.

M0, M1, and M3 membranes without coating or with a coating of FN/Col I/BSA contained clusters of Calu-3 cells (Figure 1). Cells grew poorly on all M0 membranes, as was expected since these membranes were not porous. The number of cells on the M0 membrane with L-DOPA+FN/Col I/BSA coating was higher than on the other M0 membranes, but cell density was still low, and the cells and nuclei were relatively large. Cell adhesion on M1 membranes with L-DOPA or L-DOPA+FN/Col I/BSA was better than on the other M1 membranes. On the M1 membrane coated with L-DOPA+FN/Col I/BSA, the cell density was higher than on the M1 membrane provided with L-DOPA coating alone. On the latter membrane, cells were not confluent, and both the cells and their nuclei were large. The positive effect of L-DOPA coating on Calu-3 cell adhesion and proliferation was most noticeable with $\mathrm{M} 3$ membranes coated with L-DOPA or L-DOPA+FN/Col I/BSA, which were completely covered with cells, especially for the latter coating. Moreover, these cell layers resembled those on PET membranes, both in cell density and size of the cells and their nuclei. Cell layers on PET membranes were all confluent, 


\section{- Chapter 6 -}

Airway epithelial-endothelial cell culture model on PTMC membranes

dense, and there was no difference in the size of the nuclei between the coating conditions. Cell coverage on TCPS was also homogeneous regardless of the coating, and samples were quite confluent. However, cell numbers were lower on TCPS than on PET since the cells on TCPS were relatively large. Moreover, some of the cells lifted off the TCPS during culturing.

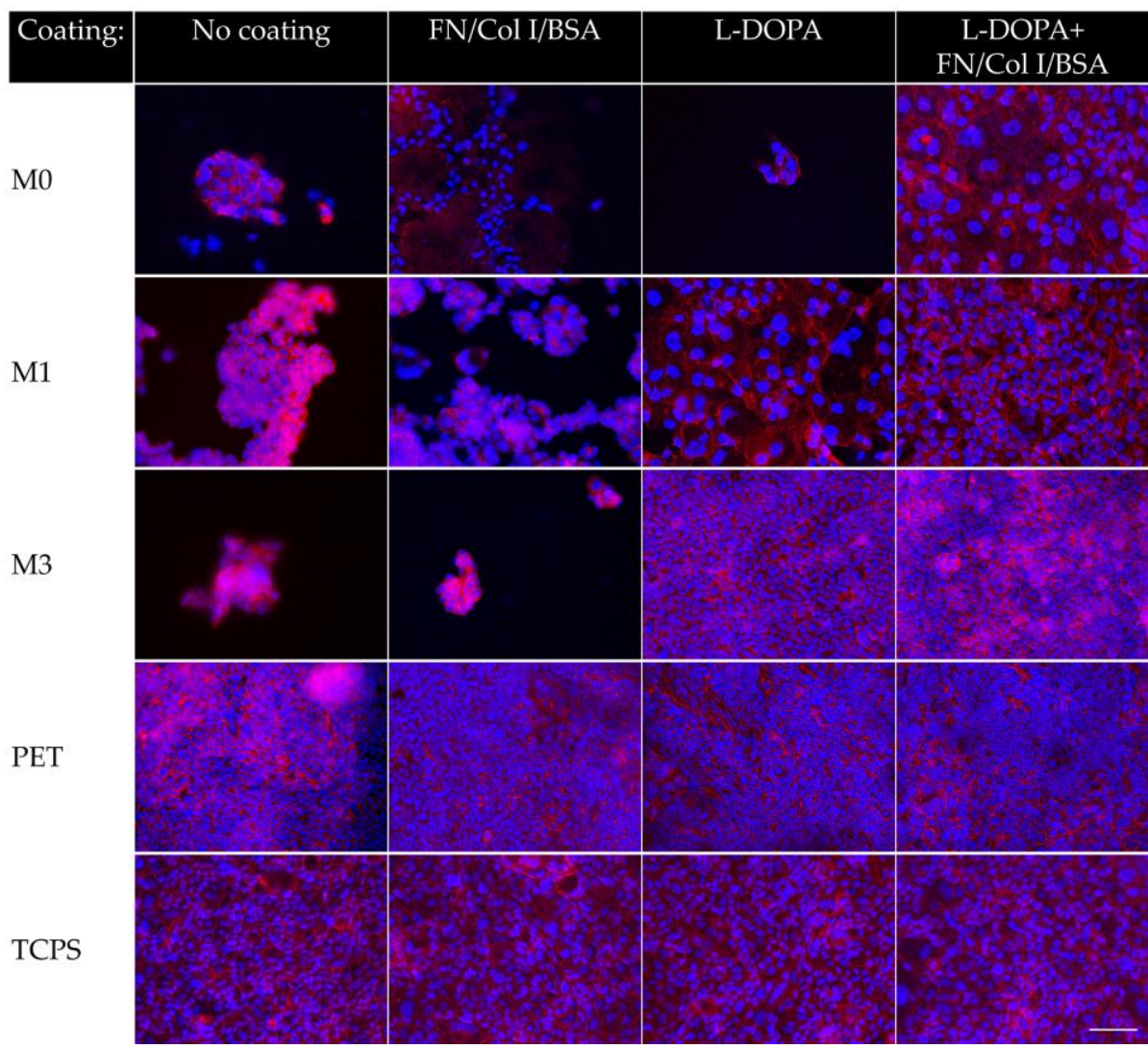

Figure 1. Cell nucleus (DAPI, blue) and F-actin (red) staining of Calu-3 cells grown for 8 days submerged and 2 weeks at air-liquid interface (ALI) on poly(trimethylene carbonate) (PTMC) and poly(ethylene terephthalate) (PET) membranes with different coatings. The latter membranes as well as tissue culture-treated polystyrene (TCPS) wells in which the cells were cultured submerged for 23 days, were used as references. Magnification: 200x. Scale bar: $100 \mu \mathrm{m}$, applies to all images. Samples originated from one experiment $(\mathrm{N}=1)$. 
The low adhesion and growth of Calu-3 cells on the non-coated and FN/Col I/BSA-coated PTMC membranes is probably caused by PEO in the membranes, which is non-cell adhesive [29-31] and inhibits protein adsorption on surfaces [31]. In contrast, L-DOPA stimulates adsorption of proteins $[25,26]$. Thus, adsorption of proteins from the FN/Col I/BSA coating or the cell culture medium was most probably improved on the membranes coated with L-DOPA, which resulted in a higher and more homogeneous cell coverage on these membranes.

The apparently large size of Calu-3 cells and their nuclei on some M0 and M1 membranes could be due to swelling or flattening of the cells and was probably caused by an external stimulus or lack thereof. For example, hyperoxia has been found to cause swelling of the nuclei of Calu-3 cells [32]. Although our cells were not cultured in hyperoxia, the surviving cells on the non-porous M0 membranes must have experienced a lot of stress due to a lack of nutrients. In addition, in our cultures, large nuclei and cells were not seen in dense monolayers or clusters. Thus, cells on the M0 and some of the M1 membranes probably experienced a lack of nutrients and/or insufficient cell-cell contact. This probably resulted in swelling or flattening of the cells (and nuclei), the latter as an attempt to receive more nutrients or improve cell-cell contact. Cells which received insufficient nutrients were probably only capable of staying adhered to the membranes because of the robustness of the cells.

Calu-3 cells developed cell layers on both M1 and M3 membranes with the L-DOPA+FN/Col I/BSA coating. Cultures on M3 membranes had higher cell densities than the cultures on M1 membranes, which is probably due to the higher permeability of the M3 membranes compared to the M1 membranes. The PET membranes performed better than the M1 membranes, despite having lower water permeance than the latter (i.e. 3,000 vs. $17,000 \mathrm{~L} /\left(\mathrm{m}^{2} \cdot \mathrm{h} \cdot \mathrm{bar}\right)$, respectively) [23]. Probably, the pore distribution of PET membranes was more homogeneous than that of M1 membranes. As a result, cell layers on the M1 membranes were less confluent than on PET membranes. In contrast, the cultures on M3 


\section{- Chapter 6 -}

Airway epithelial-endothelial cell culture model on PTMC membranes

membranes closely resembled the cultures on PET membranes. Therefore, we continued with M3 membranes and kept PET membranes as a reference. Moreover, all membranes were coated with L-DOPA+FN/Col I/BSA in further experiments.

\subsection{ZO-1 and CD31 staining of Calu-3 cells and LMVECs on PTMC membranes}

Co- and mono-cultures of Calu-3 cells and LMVECs on M3 and PET membranes were stained for ZO-1 and CD31 (Figure 2). Cells were cultured submerged for three weeks in the case of the LMVECs or eight days submerged, followed by two weeks at ALI in the case of the Calu-3 cells.

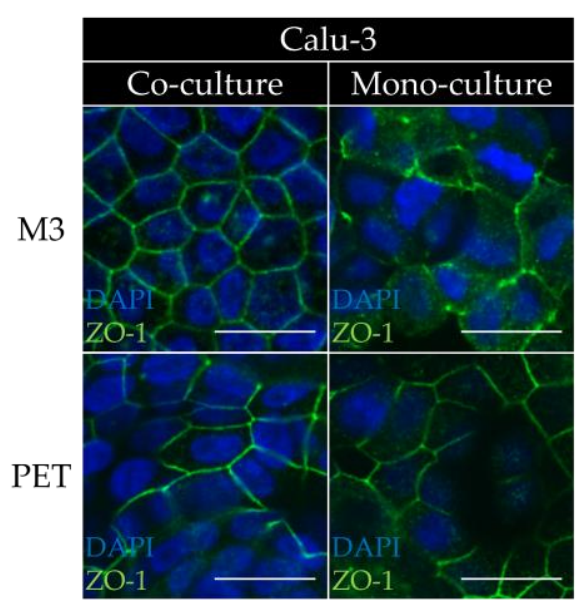

(A)

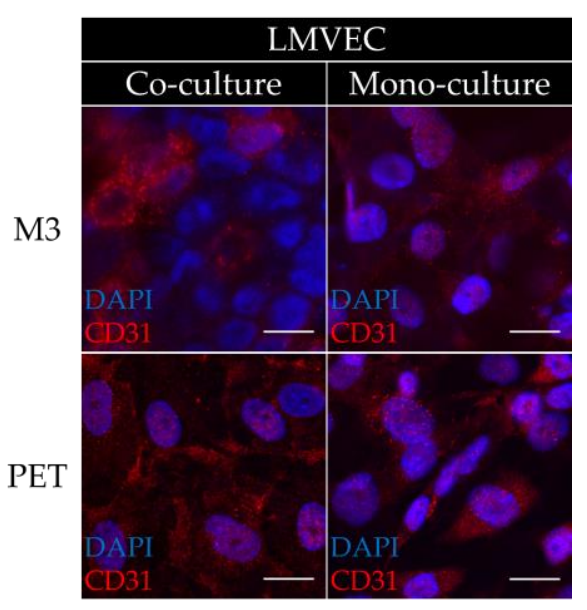

(B)

Figure 2. Staining of the tight junction protein Zonula Occludens 1 (ZO-1) and the endothelial marker CD31 of co- and mono-cultures of Calu-3 cells and human lung microvascular endothelial cells (LMVECs) in inserts on M3 membranes. Cells grown on inserts with PET membranes were used as controls. (A) Calu-3 cells were cultured and submerged for 8 days, followed by 2 weeks of culture at ALI. Cells are stained for the cell nucleus (blue) and tight junctions (ZO-1, green), magnification: 3,200x, scale bar: $20 \mu \mathrm{m}$. (B) LMVECs after 3 weeks of submerged culture. Cells are stained for the cell nucleus (blue) and CD31 (red), magnification: 1,600x, scale bar: $20 \mu \mathrm{m}$. Images are representative for observations of samples from two independent experiments (total $\mathrm{N}=3$ ). 
Calu-3 cells formed confluent cell layers on the M3 membranes and had a typical cobblestone phenotype [33] with generally little cytoplasm (Figure 2A). Cells showed good ZO-1 expression. ZO-1 expression in Calu-3 cells of the co-cultures on PTMC and PET membranes appeared to be somewhat more localised at the cell membrane compared to that in Calu3 mono-cultures. This requires confirmation in future studies using more objective, quantitative approaches. Nevertheless, these observations may be explained by interactions mediated by soluble factors, since such factors secreted by human umbilical vein endothelial cells (HUVECs) were reported to increase the production of the TJ-associated protein occludin in human bronchial epithelial-derived cells (16HBE 14o-cells) [11]. Cultures of Calu-3 cells on PET membranes were very similar to those on M3 membranes in terms of nucleus size, and the shape, size, and density of cells (Figure 2A).

LMVECs on the M3 membranes were stained for the protein CD31 (Figure 2B). Cells adhered well to the M3 membranes and expressed CD31 but did not form a confluent monolayer. The expression of CD31 was diffuse without a pronounced staining at the cell membrane. Cell densities and CD31 expression were similar between LMVECs in co- and monocultures and did not differ between M3 and PET membranes. Weppler et al. found that mono-cultures of HUVECs cultured on porous membranes showed a pronounced CD31 expression at their cell membranes, while the expression was more diffuse in co-cultures with lung epithelial cells, including Calu-3 cells, on the other side of porous membranes [34]. Moreover, while cell layers of HUVEC mono-cultures were confluent, HUVECs in co-cultures did not reach full confluency. These results are in line with the dispersed CD31 and incomplete confluency of LMVECs in our co-cultures. However, this does not explain the similar results seen in the LMVEC mono-cultures. The diffuse expression of CD31 and lack of localisation at the membrane in LMVECs of both co- and mono-cultures could be an indication of EndoMT. However, we did not find signs of EndoMT in terms of $\alpha$-SMA expression (see Section 3.5 on EMT and EndoMT marker expression). 


\subsection{Live/dead staining of Calu-3 cells and LMVECs cultured on PTMC membranes}

The viability of the co- and mono-cultures of Calu-3 cells and LMVECs on M3 and PET membranes after three weeks of submerged/ALI culture was determined by a live/dead staining (Figure 3). Cell viability was high for both Calu-3 cells and LMVECs on M3 membranes with limited cell death, as shown by the green calcein and magenta ethidium homodimer I signals, respectively. While the level of calcein signal differed between individual Calu-3 cells in mono-cultures, Calu-3 cells in co-cultures showed a very consistent and overall higher calcein signal. Moreover, Calu-3 cell death was lower in co-cultures than mono-cultures. There was little difference in the level of calcein signal between individual LMVECs of mono-cultures on M3 membranes. This was also found for LMVECs of co-cultures, but the calcein signal was more diffuse than in monocultures. The LMVECs in mono-cultures probably contracted somewhat during the assay since they were sensitive to the staining procedure, while those in co-cultures remained spread. Prior to the assay, LMVECs had probably attained better cell adhesion in co-cultures than in monocultures due to soluble factors in the co-cultures.

In general, the calcein and ethidium homodimer I signals of cultures on PET membranes were similar to those on M3 membranes (Figure 3). LMVECs of co-cultures on PET membranes showed a more dispersed calcein signal than those on M3 membranes or the LMVEC monocultures. This dispersed calcein signal was probably present because calcein-positive Calu-3 cells on the other side of the transparent PET membranes were also visible in the case of co-cultures.

These data indicate a good cell viability on M3 membranes, which was better in co-cultures than in mono-cultures. As described before, it is known that there is paracrine crosstalk between the lung epithelium and endothelium $[10,11]$. Probably, the lower Calu-3 cell death in our cocultures compared to the mono-cultures was caused by soluble factors. 
- Chapter 6 -

Airway epithelial-endothelial cell culture model on PTMC membranes

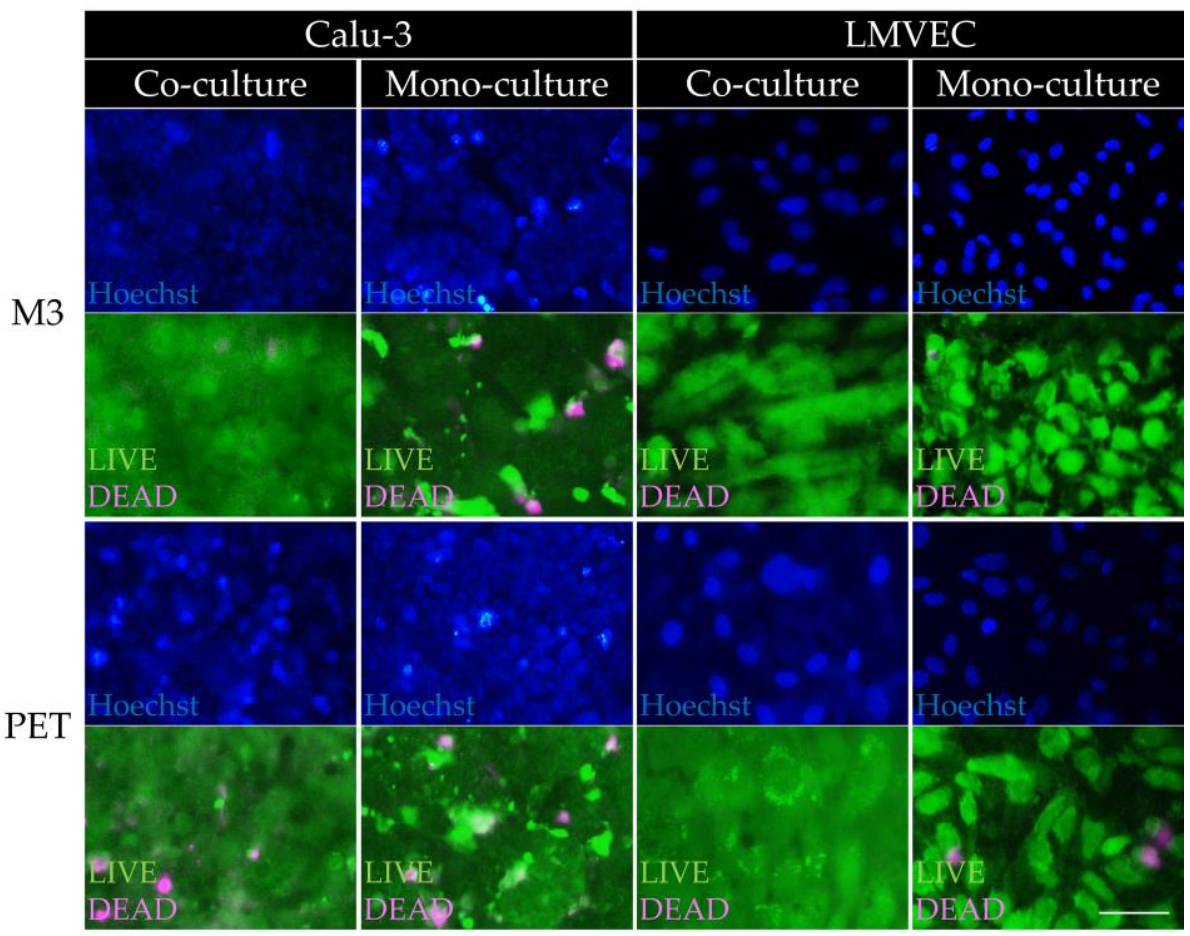

Figure 3. Live/dead staining of co- and mono-cultures of Calu-3 cells and LMVECs on M3 membranes. Cells on inserts with PET membranes served as controls. Calu-3 cells were cultured submerged for 8 days, after which they were cultured at ALI for 2 weeks. LMVECs were cultured submerged throughout the experiment. Images show the cell nuclei (Hoechst: blue), and the living (calcein: green) and dead cells (ethidium homodimer I: magenta). Magnification: 400x. Scale bar: $50 \mu \mathrm{m}$, applies to all images. Images are representative for observations of samples from two independent experiments (total $\mathrm{N}=3$ ).

\subsection{Electrical resistance and permeability for FITC-dextran of}

\section{Calu-3 cell and LMVEC co-cultures on PTMC membranes}

The barrier function of the Calu-3 and LMVEC CO- and mono-cultures on M3 and PET membranes was assessed by the electrical resistance and apparent permeability of $4 \mathrm{~kg} / \mathrm{mol}$ FITC-dextran (Figure 4 ). M3 and PET membranes without cells were included as controls. 
- Chapter 6 -

Airway epithelial-endothelial cell culture model on PTMC membranes

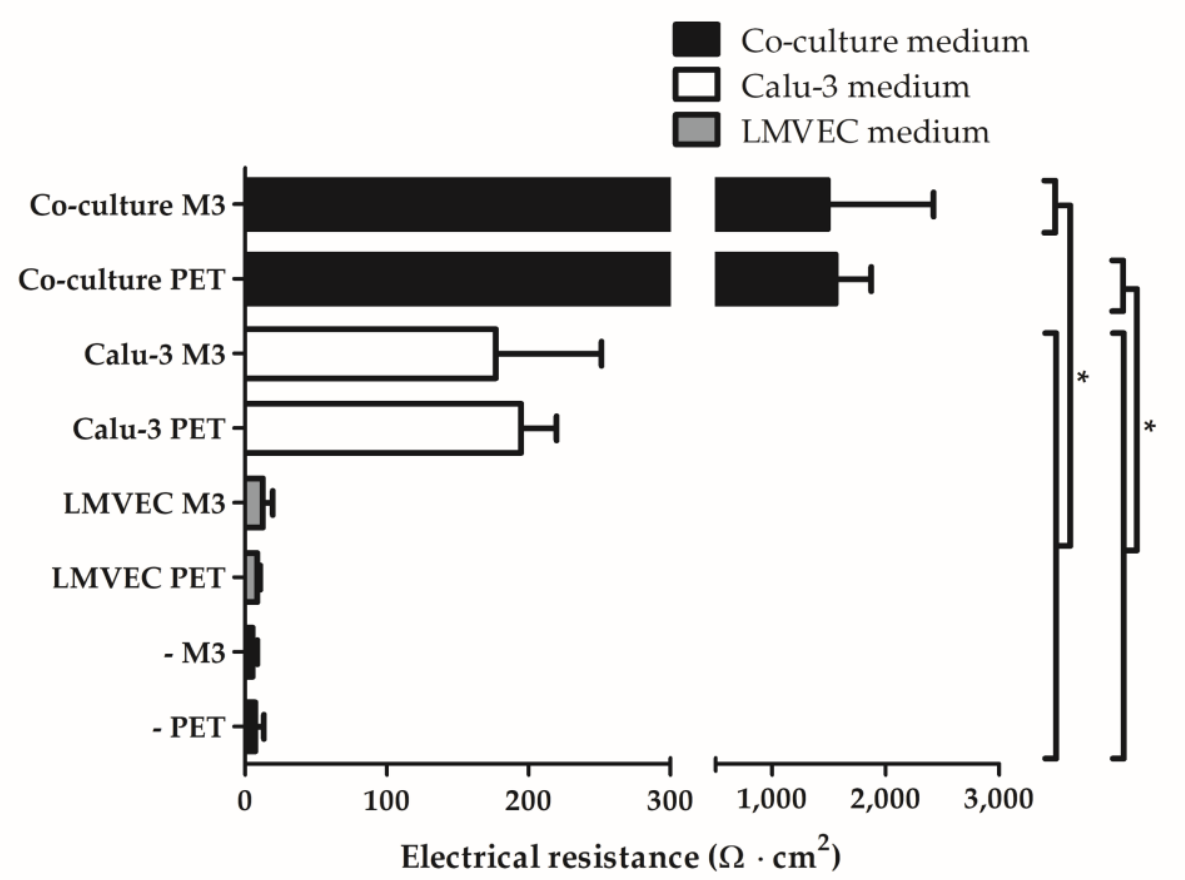

(A)

Co-culture medium

$\square$ Calu-3 medium

$\square$ LMVEC medium

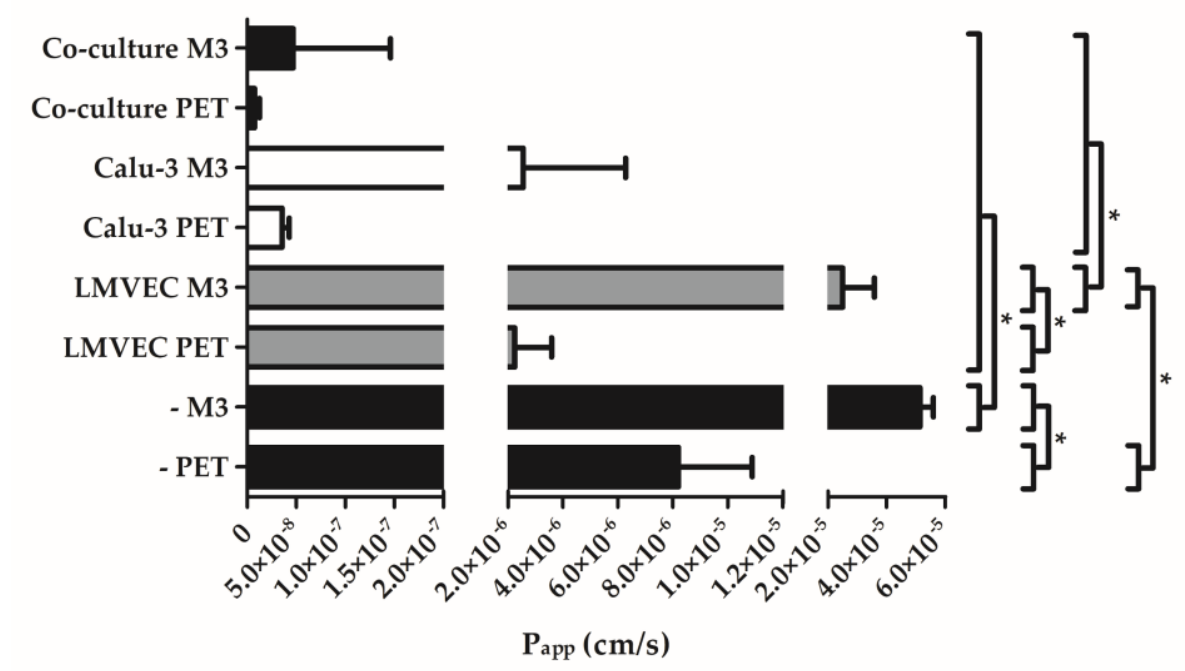

(B) 
Figure 4. Electrical resistance and Papp of fluorescein isothiocyanate-dextran (FITC-dextran) diffusion across co- and mono-cultures of Calu-3 cells and LMVECs on M3 and PET membranes. Calu-3 cells were cultured submerged for 8 days, followed by 2 weeks at ALI. (A) Electrical resistance and (B) FITC-dextran permeability assays were performed on inserts with M3 or PET membranes containing either no cells, Calu-3 cells, LMVECs, or co-cultures. Capped lines with asterisks depict significant differences, $\mathrm{P}<0.05$. Samples originated from two independent experiments (total $\mathrm{N} \geq 3$ for membranes without cells and $\mathrm{N} \geq 7$ for samples with cells). The electrical resistance and FITC-dextran permeability of membranes without cells in Calu-3 and LMVEC medium (data not shown) were similar to those of respective samples in co-culture medium.

Co-cultures had a good barrier function on M3 membranes, as indicated by a high electrical resistance and low permeability for FITC-dextran, i.e. $1,490 \Omega \cdot \mathrm{cm}^{2}$ and $4.7 \cdot 10^{-8} \mathrm{~cm} / \mathrm{s}$, respectively (Figure 4). Co-cultures on M3 membranes had much higher electrical resistance than Calu-3 cell and LMVEC mono-cultures and M3 membranes without cells, i.e. 1,490 vs. 177, 13, and $6 \Omega \cdot \mathrm{cm}^{2}$, respectively (Figure 4A). The FITC-dextran permeability of co-cultures was lower than that of LMVEC mono-cultures and M3 membranes without cells, i.e. $4.7 \cdot 10^{-8}$ vs. $2.5 \cdot 10^{-5}$ and $5.1 \cdot 10^{-5}$ $\mathrm{cm} / \mathrm{s}$, respectively (Figure 4B). Statistical analysis without the latter two conditions showed that FITC-dextran permeability of co-cultures was also significantly lower than that of Calu-3 mono-cultures on M3 membranes $\left(2.5 \cdot 10^{-6} \mathrm{~cm} / \mathrm{s}\right)$.

Calu-3 mono-cultures on M3 membranes had a significantly better barrier function in terms of FITC-dextran permeability than LMVEC monocultures and M3 membranes without cells. Moreover, statistical analysis of the electrical resistance without the very high values of the co-cultures showed a higher electrical resistance of the Calu-3 mono-cultures than of the LMVEC mono-cultures and M3 membranes without cells. In general, LMVEC mono-cultures had a very poor barrier function. Although they had a lower permeability for FITC-dextran than M3 membranes without cells, the electrical resistance was similar in both cases. The difference between the assays can be explained by the much larger size of FITCdextran $(4 \mathrm{~kg} / \mathrm{mol})$ used in the permeability assay, compared to the ions 
and charged molecules forming an electrical current in the electrical resistance assay.

There were no differences in electrical resistance between cell cultures on M3 and PET membranes. This indicates that the higher water permeance of the M3 membranes compared to the PET membranes, as determined by us before [23], did not influence the formation of cell layers on these membranes. This fits well with the immunofluorescence data (Figure 2). Co-cultures on M3 and PET membranes showed similar results in the FITC-dextran permeability assay. However, the permeability for FITCdextran of Calu-3 cell and LMVEC mono-cultures was higher on M3 membranes than on PET membranes. This is consistent with the higher FITC-dextran permeability of bare M3 membranes compared to PET membranes without cells, which is in agreement with their water permeance [23]. These data indicate that diffusion of the FITC-dextran molecules was already hampered by the relatively low permeance of the PET membranes, while the ions and small molecules involved in the electrical current were not influenced. Consequently, there were differences between mono-cultures on M3 and PET membranes in terms of permeability for FITC-dextran, but not in terms of electrical resistance. In other studies, with Calu-3 cell mono-cultures, similar FITC-dextran permeabilities were reported as in our study, indicating a good barrier function of our Calu-3 cell mono-cultures [7,8]. Moreover, the electrical resistance data of our Calu- 3 cell mono-cultures $\left(177 \Omega \cdot \mathrm{cm}^{2}\right.$ and $195 \Omega$. $\mathrm{cm}^{2}$ on M3 and PET membranes, respectively) are in line with literature [4,6-9,35,36]. Values of $50-2,500 \Omega \cdot \mathrm{cm}^{2}$ have been reported for Calu-3 cell layers, which were dependent on factors such as culture conditions (e.g. submerged or ALI culture) and measuring equipment $[4,6-9,35,36]$. The poor barrier function of LMVEC mono-cultures found by us has also been reported in other studies using endothelial cells [35-37], including LMVECs [36].

Our data showed that our system has better barrier function for the cocultures than the mono-cultures, which is in agreement with electrical resistance data of co- and mono-cultures of Calu-3 and endothelial cells 
using different support structures and membranes reported in literature [35,36]. These studies reported electrical resistances of 1,352 [35] and $>1,000 \Omega \cdot \mathrm{cm}^{2}$ [36] for the co-cultures, similar to that of our co-cultures on M3 and PET membranes (1,490 and 1,560 $\Omega \cdot \mathrm{cm}^{2}$, respectively). However, the difference between co-cultures and Calu- 3 cell mono-cultures in those studies was smaller than the difference found by us, because of the higher electrical resistance of Calu-3 cell mono-cultures in the former studies $[35,36]$ compared to our study. Formally, we cannot exclude that the different medium used for the co- and mono-cultures has contributed to the difference between them. However, since the co-culture medium was a mix of the epithelial and endothelial medium, this unlikely fully explains the difference.

The better barrier function of our co-cultures compared to the monocultures indicates a synergistic interaction between the Calu-3 cells and LMVECs in co-cultures. Based on other reports in the literature, this effect is most probably mediated by soluble factors secreted by the cultured cells rather than epithelial-endothelial cell-cell contact [10,11]. Bärnthaler et al. showed that A549 alveolar epithelial cells secreted factors which improved the barrier function of LMVECs [10]. In contrast, Chowdhury et al. found a paracrine effect of HUVECs on 16HBE 14o-epithelial cells, but not the other way around [11].

These data illustrate a complex crosstalk between epithelium and endothelium, which is not fully understood yet and probably depends on multiple factors. These studies support our observations, which emphasise the need for a co-culture of lung and endothelial cells to better mimic lung epithelial-endothelial barriers.

\subsection{Vimentin and $\alpha-S M A$ expression of Calu-3 cells and LMVECs on PTMC membranes}

The development of EMT and EndoMT of the cells in the model was assessed by staining Calu-3 cells, LMVECs, and SMCs on M3 and PET membranes for EMT and EndoMT markers (Figure 5). 


\section{- Chapter 6 -}

Airway epithelial-endothelial cell culture model on PTMC membranes

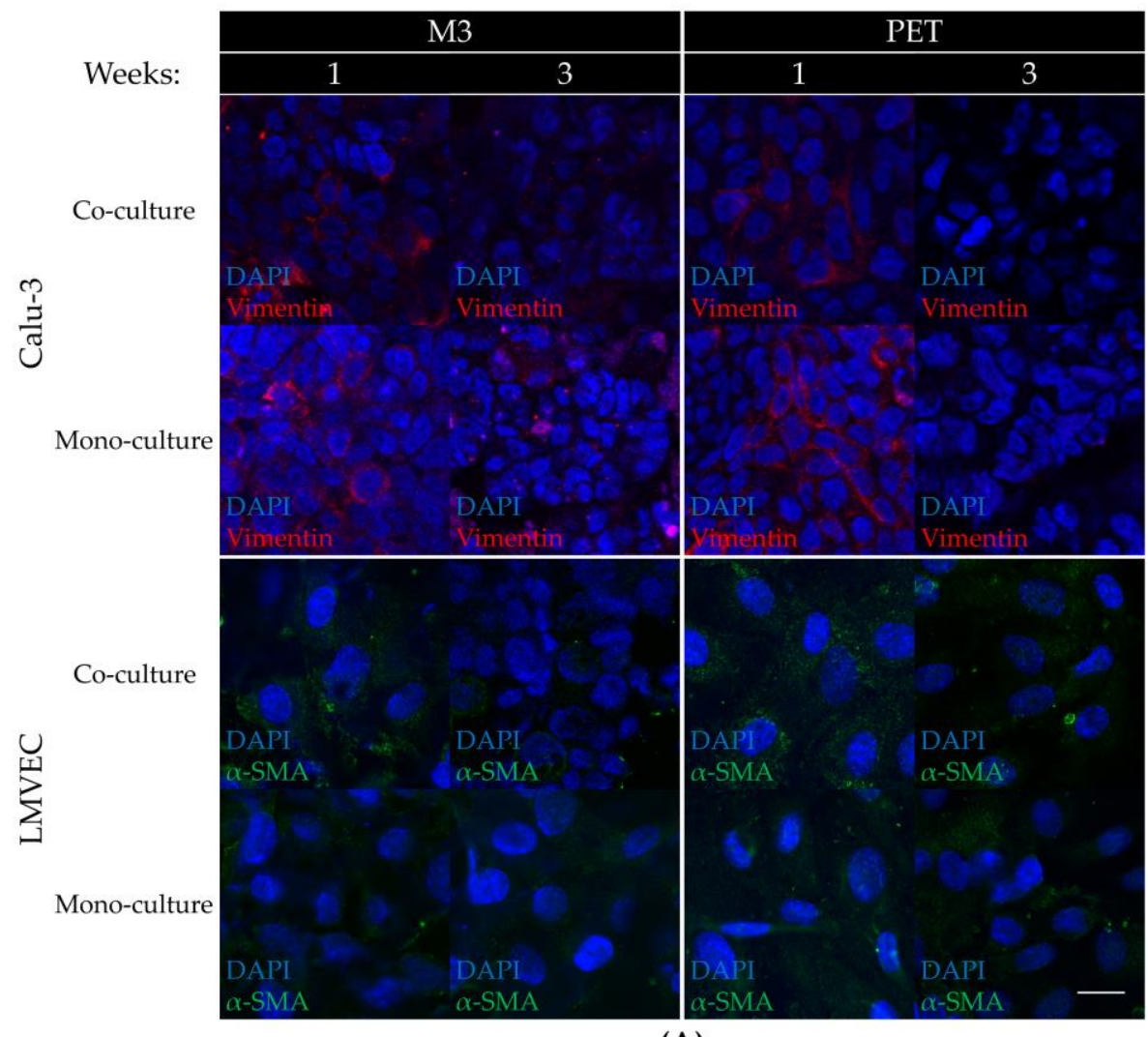

(A)

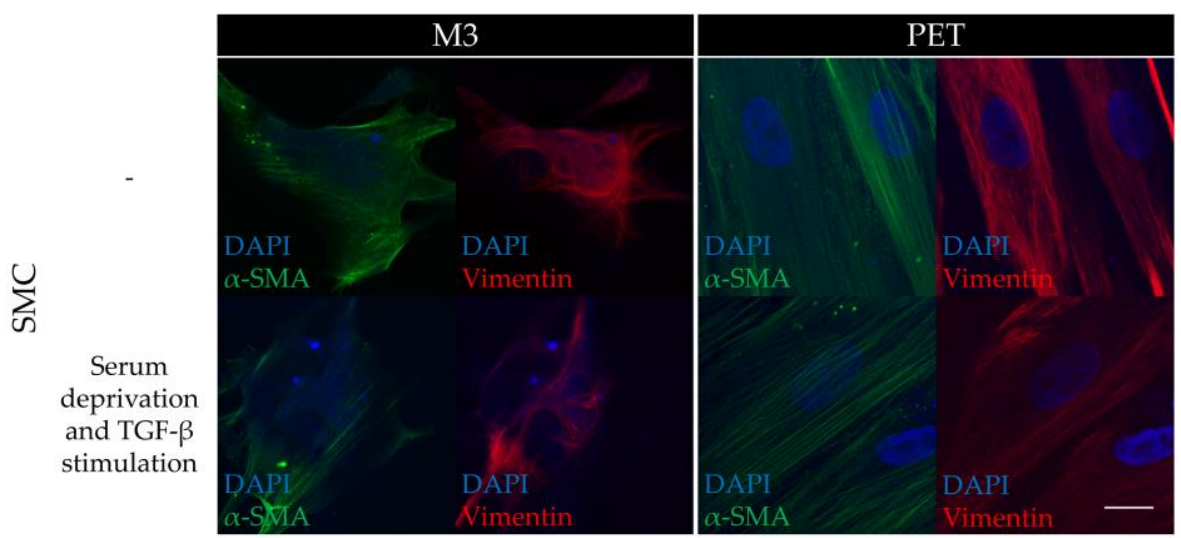

(B) 
Figure 5. Staining for epithelial-to-mesenchymal (EMT) and endothelial-tomesenchymal (EndoMT) markers of cells on M3 and PET membranes. (A) Coand mono-cultures of Calu-3 cells and LMVECs were cultured in inserts on M3 or PET membranes. Cells were cultured submerged for 1 week, after which they were either fixed or cultured for 2 additional weeks (at ALI in the case of Calu-3 cells) and fixed. Calu-3 cells were stained for the cell nucleus (blue) and vimentin (red). LMVECs were stained for the cell nucleus (blue) and $\alpha$-SMA (green). Magnification: 1,600x. Scale bar: $20 \mu \mathrm{m}$, applies to all images. (B) SMCs were stained as positive controls for mesenchymal marker expression. They were grown submerged for 5 days on M3 or PET membranes. Some cells were then serum-deprived and stimulated with transforming growth factor $\beta$ (TGF- $\beta$ ). All samples containing human smooth muscle cells (SMCs) were fixed on day 8 . SMCs were stained for the cell nucleus (blue), $\alpha$-SMA (green), and vimentin (red). Magnification: 1,600x. Scale bar: $20 \mu \mathrm{m}$, applies to all images. Images are representative for observations of multiple samples from one experiment (total $\mathrm{N}=3$ ).

Expression of vimentin, an intermediate filament protein in mesenchymal cells such as fibroblasts and SMCs, and a late marker for advanced stages of EMT, was used to investigate EMT in the Calu-3 cells (Figure 5A). $\alpha$ SMA, a protein abundantly present in microfilaments of myofibroblasts, SMCs, and other mesenchymal cells, was investigated to assess EndoMT in the LMVECs (Figure 5A). SMCs served as positive controls for mesenchymal marker expression. Serum deprivation and TGF- $\beta$ stimulation were used to upregulate the expression of vimentin and $\alpha$ SMA in SMCs.

Calu-3 cells in co- and mono-cultures on M3 membranes showed little expression of vimentin after seven days of submerged culture and two weeks of additional culture at ALI, i.e. three weeks in total (Figure 5A). We did not observe differences in expression of vimentin between cells cultured on PTMC and PET membranes, suggesting that possible development of EMT was not associated with culture of Calu-3 cells on PTMC membranes. Expression of vimentin was lower after ALI culture than after seven days of submerged culture. As Calu-3 cells acquire several characteristics similar to native epithelium when cultured at ALI 
$[7,8]$, the additional culture at ALI probably led to similar changes in our cultures, including a decrease in vimentin expression. LMVECs in co- and mono-cultures on M3 membranes showed a faint expression of $\alpha$-SMA evenly throughout the cytoplasm, which did not change between one and three weeks of culture, and was comparable to that of LMVECs on PET membranes (Figure 5A).

Unstimulated and stimulated SMCs on M3 and PET membranes expressed high amounts of vimentin and $\alpha$-SMA, and both proteins showed a filamentous organisation (Figure 5B). SMCs on M3 membranes were smaller and less spindle-like, with less filamentous organisation of vimentin and $\alpha$-SMA and less distinct cell nuclei than SMCs on PET membranes. This can be explained by the lower stiffness of M3 compared to PET membranes, in agreement with literature [38]. This indicates that, even without external mechanical actuation of the flexible membrane, the lower stiffness of the PTMC membrane can already invoke different cell responses than the PET membranes.

The expression of vimentin and $\alpha$-SMA was much higher in SMCs than in any of the Calu-3 and LMVEC co- and mono-cultures. Moreover, vimentin and $\alpha$-SMA in Calu- 3 cells and LMVECs lacked the filamentous organisation found in the SMCs (Figure 5A,B). Togami et al. reported that Calu-3 cells express some vimentin under baseline conditions without a loss of epithelial characteristics or signs of attaining a migratory state [39]. This was indicated by a high expression of the epithelial marker Ecadherin and TJ-related Claudin 1, 3, and 5 in the cells. Francescone et al. showed that human microvascular endothelial cells, which expressed typical endothelial markers and had the capacity for network formation, expressed a small amount of $\alpha$-SMA by default [40]. Thus, using vimentin as a late marker for advanced stages of EMT, we did not obtain evidence for development of EMT in Calu-3 cells as a result of culturing on the membranes. Similarly, using $\alpha$-SMA as a marker for EndoMT, there were no signs of EndoMT of the LMVECs in our cultures. 


\subsection{Calu-3 cell and LMVEC co-cultures on PTMC membranes as a functional barrier model}

Our in vitro airway epithelial-endothelial model consisting of Calu-3 cells and LMVECs on flexible porous PTMC membranes showed good ZO-1 staining in Calu-3 cells, and LMVECs were CD31-positive. Cells had high viability, and barrier function was comparable to similar cultures in literature, which confirmed the functionality of the presented model. Moreover, it offers possibilities for extension. Films and scaffolds based on crosslinked PTMC networks have shown mechanical properties suitable for cyclic stretch [20,22]. This most probably also holds for the M3 membranes, as they comprise crosslinked PTMC networks as well [23]. The presented airway epithelial-endothelial model could be mounted in a LOC and subjected to cyclic stretch and/or membrane bending to mimic the dynamic environment in a breathing lung. Moreover, the M3 membranes were formed by EIPS, a method that could be used in conjunction with micro-moulding [41-43]. In this way, microchannel-like or semi-spherical microstructures could be introduced in the PTMC membranes to better resemble the bronchioles or alveoli, respectively $[12,44]$. Such microstructures can influence cell characteristics like orientation, proliferation, and differentiation [12,44]. Moreover, as mentioned, there is a need for both airway epithelial-endothelial and alveolar-endothelial models. This study focused on the former, but also forms a basis for expansion towards alveolar models. The presence of multiple relevant lung cell types is essential for development of more advanced lung barrier models. Besides epithelial and endothelial cells, as presented in this study, future models should also focus on implementation of fibroblasts and immune cells.

\section{Conclusions}

This study combines for the first time Calu-3 cells with LMVECs on flexible porous PTMC membranes to produce an airway epithelialendothelial model. The model displays important characteristics such as ZO-1 staining by the Calu-3 cells, CD31-positive LMVECs, high cell 
viability, and a comparable barrier function to similar co-cultures in literature. Upon culturing on PTMC membranes, Calu-3 cells and LMVECs showed no increased expression of the mesenchymal markers vimentin and $\alpha$-SMA, respectively. The data also illustrate that implementation of endothelial cells provides multiple benefits, such as an improved barrier function compared to mono-cultures. This is probably caused by soluble factors, similar to the paracrine crosstalk between epithelial and endothelial cells. Future implementation of our model in LOCs that provide cyclic stretch and/or bending of the membrane would allow mechanical stimulation of the cells. This enables studying the impact of the mechanical forces of breathing, which cannot be achieved with PET membranes. These characteristics make this airway epithelialendothelial model of Calu-3 cells and LMVECs on PTMC membranes a possible alternative model for lung research, as well as a foundation for more advanced models.

\section{References}

1. World Health Organization. The Top 10 Causes of Death. Available online: https://www.who.int/news-room/fact-sheets/detail/the-top-10-causes-of-death (accessed on 28 December 2020).

2. Van Riet, S.; Ninaber, D.K.; Mikkers, H.; Tetley, T.D.; Jost, C.R.; Mulder, A.A.; Pasman, T.; Baptista, D.; Poot, A.A.; Truckenmüller, R.; et al. In vitro modelling of alveolar repair at the air-liquid interface using alveolar epithelial cells derived from human induced pluripotent stem cells. Sci. Rep. 2020, 10, 5499. DOI: 10.1038/s41598-020-62226-1.

3. Nikolic, M.; Sustersic, T.; Filipovic, N. In vitro models and on-chip systems: Biomaterial interaction studies with tissues generated using lung epithelial and liver metabolic cell lines. Front. Bioeng. Biotechnol. 2018, 6, 120. DOI: 10.3389/fbioe.2018.00120.

4. Forbes, B.; Ehrhardt, C. Human respiratory epithelial cell culture for drug delivery applications. Eur. J. Pharm. Biopharm. 2005, 60, 193-205. DOI: 10.1016/j.ejpb.2005.02.010.

5. Derk, R.; Davidson, D.C.; Manke, A.; Stueckle, T.A.; Rojanasakul, Y.; Wang, L. Potential in vitro model for testing the effect of exposure to nanoparticles on the lung alveolar epithelial barrier. Sens. Biosensing. Res. 2015, 3, 38-45. DOI: 10.1016/j.sbsr.2014.12.002.

6. Dierks, A.; Bader, A.; Lehrich, T.; Ngezahayo, A. Stimulation of the $\mathrm{A}_{2 в}$ adenosine receptor subtype enhances Connexin26 hemichannel activity in small airway 
epithelial cells. Cell. Physiol. Biochem. 2019, 53, 606-622. DOI: 10.33594/000000160.

7. Grainger, C.I.; Greenwell, L.L.; Lockley, D.J.; Martin, G.P.; Forbes, B. Culture of Calu-3 cells at the air interface provides a representative model of the airway epithelial barrier. Pharm. Res. 2006, 23, 1482-1490. DOI: 10.1007/s11095-006-02550 .

8. Kreft, M.E.; Jerman, U.D.; Lasič, E.; Hevir-Kene, N.; Rižner, T.L.; Peternel, L.; Kristan, K. The characterization of the human cell line Calu-3 under different culture conditions and its use as an optimized in vitro model to investigate bronchial epithelial function. Eur. J. Pharm. Sci. 2015, 69, 1-9. DOI: 10.1016/j.ejps.2014.12.017.

9. Srinivasan, B.; Kolli, A.R.; Esch, M.B.; Abaci, H.E.; Shuler, M.L.; Hickman, J.J. TEER measurement techniques for in vitro barrier model systems. J. Lab. Autom. 2015, 20, 107-126. DOI: 10.1177/2211068214561025.

10. Bärnthaler, T.; Maric, J.; Platzer, W.; Konya, V.; Theiler, A.; Hasenöhrl, C.; Gottschalk, B.; Trautmann, S.; Schreiber, Y.; Graier, W.F.; et al. The role of PGE2 in alveolar epithelial and lung microvascular endothelial crosstalk. Sci. Rep. 2017, 7, 7923. DOI: 10.1038/s41598-017-08228-y.

11. Chowdhury, F.; Howat, W.J.; Phillips, G.J.; Lackie, P.M. Interactions between endothelial cells and epithelial cells in a combined cell model of airway mucosa: Effects on tight junction permeability. Exp. Lung Res. 2010, 36, 1-11. DOI: 10.3109/01902140903026582.

12. Chapter 2 of this thesis.

13. Huh, D.; Leslie, D.C.; Matthews, B.D.; Fraser, J.P.; Jurek, S.; Hamilton, G.A.; Thorneloe, K.S.; McAlexander, M.A.; Ingber, D.E. A human disease model of drug toxicity-induced pulmonary edema in a lung-on-a-chip microdevice. Sci. Transl. Med. 2012, 4. DOI: 10.1126/scitranslmed.3004249.

14. Kim, H.J.; Ingber, D.E. Gut-on-a-Chip microenvironment induces human intestinal cells to undergo villus differentiation. Integr. Biol. 2013, 5, 1130-1140. DOI: 10.1039/c3ib40126j.

15. Stucki, A.O.; Stucki, J.D.; Hall, S.R.; Felder, M.; Mermoud, Y.; Schmid, R.A.; Geiser, T.; Guenat, O.T. A lung-on-a-chip array with an integrated bio-inspired respiration mechanism. Lab Chip. 2015, 15, 1302-1310. DOI: 10.1039/c4lc01252f.

16. Gotoh, K.; Yasukawa, A.; Taniguchi, K. Water contact angles on poly(ethylene terephthalate) film exposed to atmospheric pressure plasma. J. Adhes. Sci. Technol. 2011, 25, 307-322. DOI: 10.1163/016942410X511114.

17. Barnes, J.M.; Przybyla, L.; Weaver, V.M. Tissue mechanics regulate brain development, homeostasis and disease. J. Cell. Sci. 2017, 130, 71-82. DOI: $10.1242 /$ jcs.191742.

18. Butcher, D.T.; Alliston, T.; Weaver, V.M. A tense situation: Forcing tumour progression. Nat. Rev. Cancer. 2009, 9, 108-122. DOI: 10.1038/nrc2544.

19. Pêgo, A.P.; Siebum, B.; Van Luyn, M.J.; Gallego y Van Seijen, X.J.; Poot, A.A.; Grijpma, D.W.; Feijen, J. Preparation of degradable porous structures based on 1,3-trimethylene carbonate and D,L-lactide (co)polymers for heart tissue engineering. Tissue Eng. 2003, 9, 981-994. DOI: 10.1089/107632703322495628. 


\section{- Chapter 6 -}

Airway epithelial-endothelial cell culture model on PTMC membranes

20. Bat, E.; Kothman, B.H.M.; Higuera, G.A.; van Blitterswijk, C.A.; Feijen, J.; Grijpma, D.W. Ultraviolet light crosslinking of poly(trimethylene carbonate) for elastomeric tissue engineering scaffolds. Biomaterials. 2010, 31, 8696-8705. DOI: 10.1016/j.biomaterials.2010.07.102.

21. Papenburg, B.J.; Schuller-Ravoo, S.; Bolhuis-Versteeg, L.A.M.; Hartsuiker, L.; Grijpma, D.W.; Feijen, J.; Wessling, M.; Stamatialis, D. Designing porosity and topography of poly(1,3-trimethylene carbonate) scaffolds. Acta Biomater. 2009, 5, 3281-3294. DOI: 10.1016/j.actbio.2009.05.017.

22. Bat, E.; Feijen, J.; Grijpma, D.W. Biodegradable elastomeric networks: Highly efficient cross-linking of poly(trimethylene carbonate) by gamma irradiation in the presence of pentaerythritol triacrylate. Biomacromolecules. 2010, 11, 2692 2699. DOI: $10.1021 / \mathrm{bm} 1007234$.

23. Chapter 5 of this thesis.

24. Laulicht, B.; Mancini, A.; Geman, N.; Cho, D.; Estrellas, K.; Furtado, S.; Hopson, R.; Tripathi, A.; Mathiowitz, E. Bioinspired bioadhesive polymers: Dopamodified poly(acrylic acid) derivatives. Macromol. Biosci. 2012, 12, 1555-1565. DOI: 10.1002/mabi.201200179.

25. Ni, M.; Teo, J.C.; Ibrahim, M.S.; Zhang, K.; Tasnim, F.; Chow, P.Y.; Zink, D.; Ying, J.Y. Characterization of membrane materials and membrane coatings for bioreactor units of bioartificial kidneys. Biomaterials. 2011, 32, 1465-1476. DOI: 10.1016/j.biomaterials.2010.10.061.

26. Chevtchik, N.V.; Fedecostante, M.; Jansen, J.; Mihajlovic, M.; Wilmer, M.; Rüth, M.; Masereeuw, R.; Stamatialis, D. Upscaling of a living membrane for bioartificial kidney device. Eur. J. Pharmacol. 2016, 790, 28-35. DOI: 10.1016/j.ejphar.2016.07.009.

27. Yeggoni, D.P.; Subramanyam, R. Binding studies of L-3,4dihydroxyphenylalanine with human serum albumin. Mol. Biosyst. 2014, 10, 3101-3110. DOI: 10.1039/c4mb00408f.

28. Van Wetering, S.; van der Linden, A.C.; van Sterkenburg, M.A.; de Boer, W.I.; Kuijpers, A.L.; Schalkwijk, J.; Hiemstra, P.S. Regulation of SLPI and elafin release from bronchial epithelial cells by neutrophil defensins. Am. J. Physiol. Lung C. 2000, 278, L51-L58. DOI: 10.1152/ajplung.2000.278.1.L51.

29. Liang, J.; Guo, Z.; Timmerman, A.; Grijpma, D.; Poot, A.A. Enhanced mechanical and cell adhesive properties of photo-crosslinked PEG hydrogels by incorporation of gelatin in the networks. Biomed. Mater. 2019, 14, 024102. DOI: 10.1088/1748-605X/aaf31b.

30. Allijn, I.; Ribeiro, M.; Poot, A.A.; Passier, R.; Stamatialis, D. Membranes for modelling cardiac tissue stiffness in vitro based on poly(trimethylene carbonate) and poly(ethylene glycol) polymers. Membranes. 2020, 10, E274. DOI: 10.3390/membranes10100274.

31. Gombotz, W.R.; Wang, G.H.; Horbett, T.A.; Hoffman, A.S. Protein adsorption to poly(ethylene oxide) surfaces. J. Biomed. Mater. Res. 1991, 25, 1547-1562. DOI: $10.1002 / \mathrm{jbm} .820251211$. 
32. Zhu, Y.; Chidekel, A.; Shaffer, T.H. Cultured human airway epithelial cells (calu3): A model of human respiratory function, structure, and inflammatory responses. Crit. Care Res. Pract. 2010, 2010, 394578. DOI: 10.1155/2010/394578.

33. Kale, S.L.; Agrawal, K.; Gaur, S.N.; Arora, N. Cockroach protease allergen induces allergic airway inflammation via epithelial cell activation. Sci. Rep. 2017, 7, 42341. DOI: 10.1038/srep42341.

34. Weppler, A.; Rowter, D.; Hermanns, I.; Kirkpatrick, C.J.; Issekutz, A.C. Modulation of endotoxin-induced neutrophil transendothelial migration by alveolar epithelium in a defined bilayer model. Exp. Lung Res. 2006, 32, 455-482. DOI: 10.1080/01902140601059463.

35. Zhang, F.; Aquino, G.V.; Dabi, A.; Bruce, E.D. Assessing the translocation of silver nanoparticles using an in vitro co-culture model of human airway barrier. Toxicol. In Vitro. 2019, 56, 1-9. DOI: 10.1016/j.tiv.2018.12.013.

36. Dekali, S.; Gamez, C.; Kortulewski, T.; Blazy, K.; Rat, P.; Lacroix, G. Assessment of an in vitro model of pulmonary barrier to study the translocation of nanoparticles. Toxicol. Rep. 2014, 1, 157-171. DOI: 10.1016/j.toxrep.2014.03.003.

37. Kazakoff, P.W.; McGuire, T.R.; Hoie, E.B.; Cano, M.; Iversen, P.L. An in vitro model for endothelial permeability: Assessment of monolayer integrity. In Vitro Cell. Dev. Biol. Anim. 1995, 31, 846-852. DOI: 10.1007/BF02634568.

38. Nagayama, K.; Nishimiya, K. Moderate substrate stiffness induces vascular smooth muscle cell differentiation through cellular morphological and tensional changes. Biomed. Mater. Eng. 2020, 31, 157-167. DOI: 10.3233/BME-201087.

39. Togami, K.; Yamaguchi, K.; Chono, S.; Tada, H. Evaluation of permeability alteration and epithelial-mesenchymal transition induced by transforming growth factor- $\beta_{1}$ in A549, NCI-H441, and Calu-3 cells: Development of an in vitro model of respiratory epithelial cells in idiopathic pulmonary fibrosis. J. Pharmacol. Toxicol. Methods. 2017, 86, 19-27. DOI: 10.1016/j.vascn.2017.02.023.

40. Francescone, R.; Scully, S.; Bentley, B.; Yan, W.; Taylor, S.L.; Oh, D.; Moral, L.; Shao, R. Glioblastoma-derived tumor cells induce vasculogenic mimicry through Flk-1 protein activation. J. Biol. Chem. 2012, 287, 24821-24831. DOI: 10.1074/jbc.M111.334540.

41. Papenburg, B.J.; Vogelaar, L.; Bolhuis-Versteeg, L.A.M.; Lammertink, R.G.H.; Stamatialis, D.; Wessling, M. One-Step fabrication of porous micropatterned scaffolds to control cell behavior. Biomaterials. 2007, 28, 1998-2009. DOI: 10.1016/j.biomaterials.2006.12.023.

42. Girones, M.; Akbarsyah, I.J.; Nijdam, W.; van Rijn, C.J.M.; Jansen, H.V.; Lammertink, R.G.H.; Wessling, M. Polymeric microsieves produced by phase separation micromolding. J. Membr. Sci. 2006, 283, 411-424. DOI: 10.1016/j.memsci.2006.07.016.

43. Vogelaar, L.; Lammertink, R.G.H.; Barsema, J.N.; Nijdam, W.; Bolhuis-Versteeg, L.A.M.; van Rijn, C.J.M.; Wessling, M. Phase separation micromolding: A new generic approach for microstructuring various materials. Small. 2005, 1, 645-655. DOI: $10.1002 /$ smll.200400128.

44. Baptista, D.; Teixeira, L.M.; Birgani, Z.T.; van Riet, S.; Pasman, T.; Poot, A.; Stamatialis, D.; Rottier, R.J.; Hiemstra, P.S.; Habibović, P.; et al. 3D alveolar in 


\section{- Chapter 6 -}

Airway epithelial-endothelial cell culture model on PTMC membranes

vitro model based on epithelialized biomimetically curved culture membranes.

Biomaterials. 2021, 266, 120436. DOI: 10.1016/j.biomaterials.2020.120436. 
- Chapter 6 -

Airway epithelial-endothelial cell culture model on PTMC membranes

Supplementary material of Chapter 6: Development of an in vitro airway epithelial-endothelial cell culture model on a flexible porous poly(trimethylene carbonate) membrane based on Calu-3 airway epithelial cells and lung microvascular endothelial cells
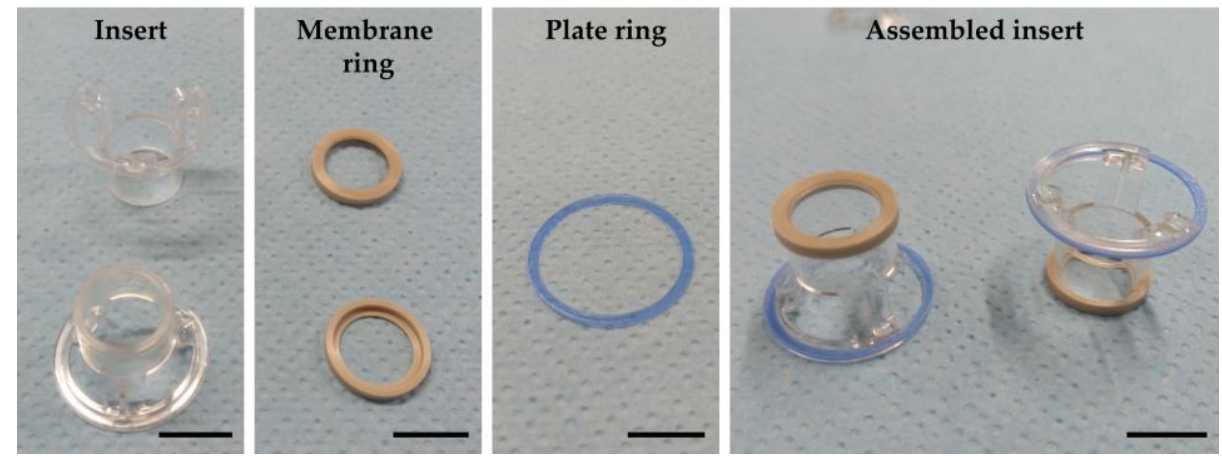

Figure S1. Cell culture inserts for the poly(trimethylene carbonate) (PTMC) membranes. Depicted are, from left to right: commercial Transwell ${ }^{\circledR}$ cell inserts with the standard poly(ethylene terephthalate) (PET) membrane removed, custom made membrane and plate rings (made from poly(ether ether ketone) (PEEK) and poly(propylene) (PP), respectively), and the assembled insert (insert with membrane and plate rings). PTMC membranes were secured to the bottom of the inserts by use of the membrane rings. The plate rings were situated between the top of the culture plate wells and the inserts, raising the latter to allow cell culture medium to reach the underside of the inserts. Scale bar: $1 \mathrm{~cm}$. 
- Chapter 6 -

Airway epithelial-endothelial cell culture model on PTMC membranes 


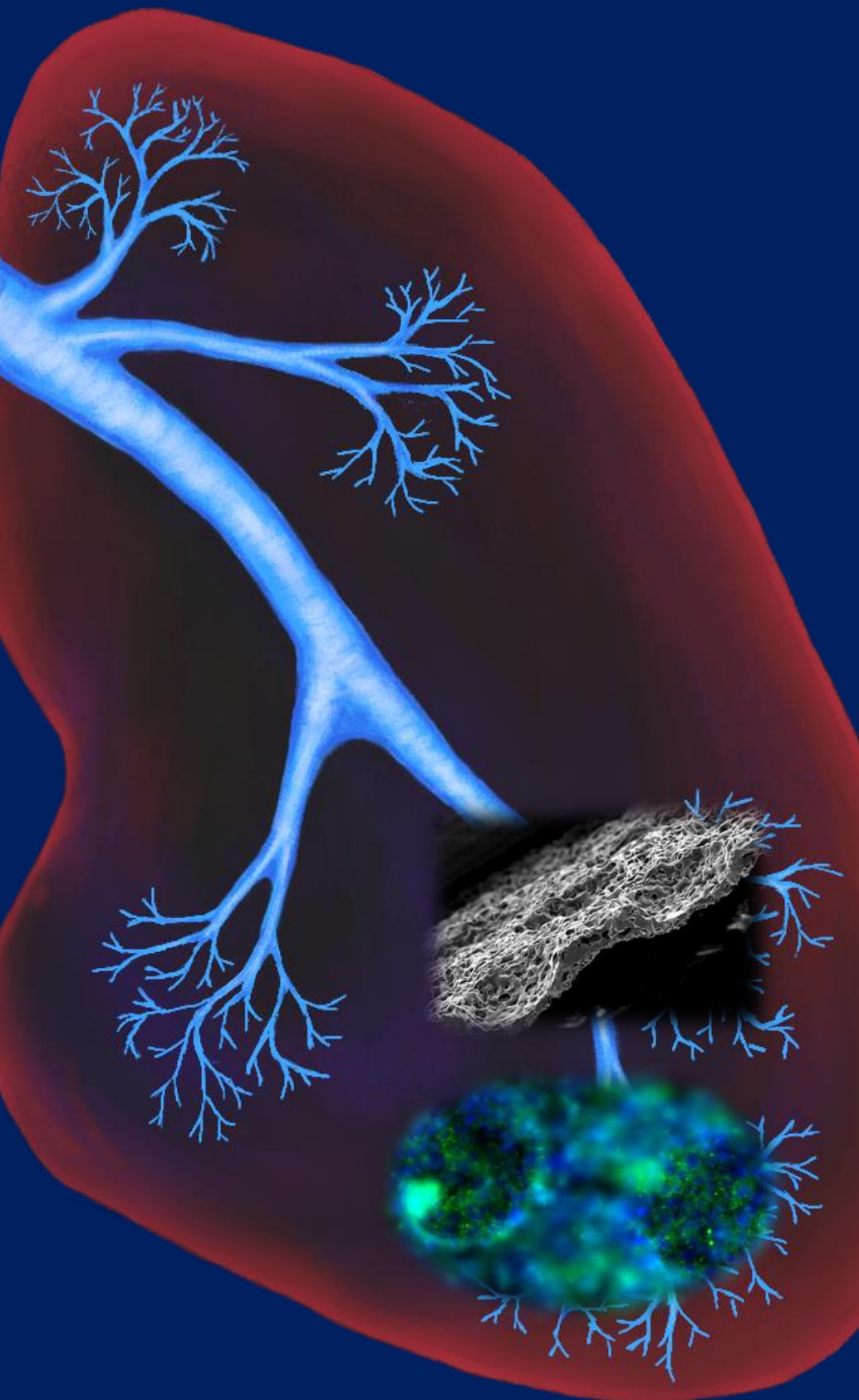




\section{- Chapter 7 -}

\section{Development of flexible porous PTMC membranes with microwells for an in vitro airway epithelial-endothelial cell culture model}

Thijs Pasman 1, Naomi M. Hamelmann 2, Jos M. J. Paulusse 2, Dimitrios Stamatialis ${ }^{1}$ and André A. Poot ${ }^{1}$

1 Technical Medical (TechMed) Centre, Department of Biomaterials Science and Technology, Faculty of Science and Technology, University of Twente, 7522 NB Enschede, The Netherlands

2 Department of Biomolecular NanoTechnology, Faculty of Science and Technology, University of Twente, 7522 NB Enschede, The Netherlands 
PTMC-microwell membranes for airway epithelial-endothelial cell model

\section{Abstract}

Lungs-on-chips (LOCs) are biomimetic in vitro lung models for developing drugs and therapies for lung diseases. In most LOCs, cells are cultured on flat membranes, however, growing evidence indicates that e.g. cell proliferation, differentiation, alignment and ECM deposition can be influenced by membrane surface topography. In this chapter, we developed for the first time polymeric membranes containing microwells mimicking the lung alveoli for in vitro lung models in cell culture inserts and LOCs. These porous membranes are prepared by combining evaporation-induced phase separation (EIPS) and micromoulding using poly(trimethylene carbonate) (PTMC). The fabricated membranes were integrated well into cell culture inserts and a commercial organ-on-chip platform, the latter allowing air- and fluid flow. Lung epithelial cells and lung microvascular endothelial cells, i.e. Calu-3 cells and LMVECs, were co-cultured on the membranes. Calu-3 cells and LMVECs were ZO-1 and ZO-1/CD31 positive, respectively, and showed good viability in both cell culture inserts and chips. Besides, high electrical resistance and low permeability of FITC-dextran of co-cultures in inserts suggested good cell barrier function. We consider this as the first step towards developing biomimetic membranes for in vitro lung models. Future work would focus on detailed investigation of the cell culture on these membranes in a LOC system.

Keywords: EIPS, micromoulding, PTMC, membranes, microwells, Calu-3 cells, LMVECs, barrier function, organ-on-chip

\section{Introduction}

Currently, lung diseases are still one of the main causes of death [1]. Unfortunately, the development of new drugs is increasingly difficult, largely due to inadequate research models. In vivo animal models have limited predictability concerning drug responses in humans and there are increasing ethical issues. In vitro models often do not accurately mimic the in vivo situation, mostly due to a lack of physiological stimuli for the cells. 
PTMC-microwell membranes for airway epithelial-endothelial cell model

In recent years, lungs-on-chips (LOCs) have addressed many limitations of in vitro lung models by introducing conditions that could better mimic the in vivo environment, such as airflow, and mechanical stimulation of the cells [2-4]. Although there are many iterations of LOCs, most of them represent the smaller airways or alveoli, the latter facilitating gas exchange between the lung and the blood. The cells are usually cultured on a flexible porous membrane made from poly(dimethyl siloxane) (PDMS) [4]. It acts as the basement membrane, a flexible extracellular matrix (ECM) between the lung epithelium and endothelium providing cell support but also facilitating transport of molecules and signalling between the cells. Although LOCs aim to mimic the alveoli and (smaller) airways such as the bronchioles, i.e. spherical and cylindrical structures, respectively, the membranes used in LOCs are flat. Growing evidence, however, indicates that e.g. cell proliferation, differentiation, alignment and ECM deposition can be influenced by small and large microstructures (i.e. smaller and larger than a cell) on the surface on which cells are cultured [4]. Cell culture constructs with hemispherical channels [5-7] and hemispherical microwells $[8,9]$ have been produced, but studies on the effect of these microstructures in the context of lung models, are limited. Thus, introducing cylindrical or spherical microstructures to in vitro lung models to mimic the (smaller) airways and alveoli is very relevant. Phase separation micromoulding $(\mathrm{PS} \mu \mathrm{M})$ is a technique to prepare polymer constructs with pores and microstructures and has been used quite extensively to fabricate biocompatible cell culture constructs $[4,10]$. $\mathrm{PS} \mu \mathrm{M}$ involves casting a polymer solution on a microstructured mould after which phase separation occurs, creating porous membranes with microstructures $[4,10,11]$. However, PS $\mu \mathrm{M}$ is usually performed using liquid-induced phase separation (LIPS), which is difficult to perform in combination with (photo-)crosslinking [12]. The latter is required for many polymers, including many flexible biomaterials, to ensure mechanical stability of the membranes and preserve the porosity and microstructures [12].

In this study, we combined for the first time evaporation-induced phase separation (EIPS) with micromoulding to develop flexible, permeable and 
PTMC-microwell membranes for airway epithelial-endothelial cell model

mechanically stable membranes with hemispherical microwells. The polymer poly(trimethylene carbonate) (PTMC) was used to prepare the membranes. Hemispherical microwells were chosen since they mimic alveoli but they can also be relevant for studies involving small airway epithelial cells since the cells would feel a similar degree of curvature in the smaller airways. Previously, we have used EIPS to fabricate flat and flexible PTMC membranes with good mechanical stability, porosity and permeability [13]. Moreover, a co-culture of Calu-3 lung airway epithelial cells and lung microvascular endothelial cells (LMVECs) was successfully established on these membranes [14]. The developed microwell $(\mu \mathrm{W})$ membranes were characterised in terms of membrane morphology, gel content, electrical resistance and apparent permeability ( $\left.\mathrm{P}_{\mathrm{app}}\right)$ of FITCdextran. Calu-3 cells and LMVECs were cultured in cell culture inserts on opposite sides of the $\mu \mathrm{W}$ and flat PTMC membranes, as well as on commercial PET membranes to create analogues of lung epithelialendothelial barriers in the smaller airways and alveoli. Electrical resistance and $\mathrm{P}_{\mathrm{app}}$ of these cultures were compared to each other and to respective membranes without cells. Furthermore, co-cultures on $\mu \mathrm{W}$ membranes were incorporated in a commercial organ-on-chip system. Co-cultures on all membranes in inserts and chips were stained for ZO-1, CD31 and cell viability.

\section{Materials and methods}

\subsection{Materials}

Trichloro( $1 H, 1 H, 2 H, 2 H$-perfluorooctyl)silane (PFOCTS) (cat. 448931) was purchased from Sigma-Aldrich, St. Louis, MO, USA. A Sylgard ${ }^{\text {TM }} 184$ Silicone Elastomer Kit (cat. DOWC634165S) was acquired from Dow Europe GMBH C/O, Wiesbaden, Germany and Poly(vinylidene fluoride) (PVDF) sheets (cat. 130-285-21) were bought from Goodfellow, Huntington, England. For the chip setup, a Fluidic Connect Pro Organon-Chip 4515 (cat. 00750), Organ-on-Chip 4515 Resealable Top Layers and Resealable Bottom Layers (cat. 00739), Organ-on-Chip middle layers 4515 Glass thickness $0.4 \mathrm{~mm}$, with adhesive without membrane (cat. 01967) and 
PTMC-microwell membranes for airway epithelial-endothelial cell model

a 'Fluidic Connect Pro ETFE connection kit' (FC_PRO_TF_KIT) were all bought from Micronit Microtechnologies, Enschede, The Netherlands. Pumpsil platinum-cured silicone tubing ( $0.5 \mathrm{~mm}$ bore, $1.6 \mathrm{~mm}$ wall) for the chips (cat. 913.AJ05.016) was purchased from Watson-Marlow Tubing, Barendrecht, The Netherlands. For additional information, other materials are listed in Section 2.1 of Chapter 5 and Section 2.1 of Chapter 6.

\subsection{Mould fabrication}

For the fabrication of the membranes, polymer casting on a mould with concave microwells would not result in deep, hollow microwells as the polymer solution would fill the microwells of the mould. Thus, a mould with convex microwells was required. However, only silicon master moulds with concave microwells could be prepared (Figure 1.I). Thus, a series of intermediate PDMS moulds (Figure 1.I-V) was used to enable hot embossing of a PVDF plate to produce a mould with convex microwells (Figure 1.VI-VII). Importantly, since the fabrication of the silicon master mould was very laborious, it was not used for hot embossing to avoid damaging the mould. Moreover, hot embossing of the PVDF plate directly on the silicon mould would result in two rigid materials on top of each other, probably requiring breaking of the silicon mould to detach it from the PVDF.

A silicon wafer was dry etched to fabricate a silicon master mould with concave hemispherical microwells of approximately $200 \mu \mathrm{m}$ in diameter and $100 \mu \mathrm{m}$ in depth. Spacing between microwells was around $100 \mu \mathrm{m}$. This master mould was then placed in a Teflon tray, after which PDMS was cast over the mould (Figure 1.I). The PDMS had a 1:10 weight ratio of curing agent and PDMS. The primary PDMS mould was then left to cure at room temperature for 3 days. The mould (Figure 1.II) had good convex microwells but could not be used for membrane production as it swelled due to the chloroform of the polymer solution. Thus, the mould was silanised by leaving both the mould and some PFOCTS adjacent to it in a covered petri dish for 20 minutes (Figure 1.III). 
PTMC-microwell membranes for airway epithelial-endothelial cell model

I. Cast primary PDMS mould

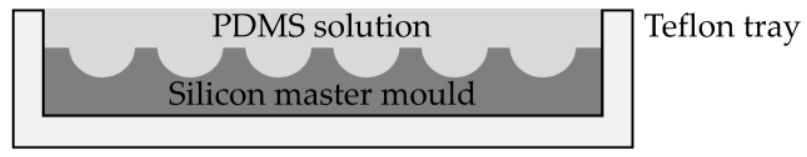

II. Primary PDMS mould

\section{PDMS}

III. Silanise PDMS

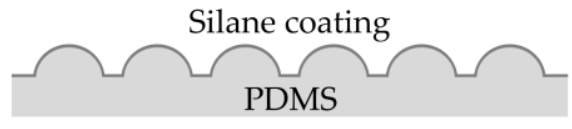

IV. Cast secondary PDMS mould

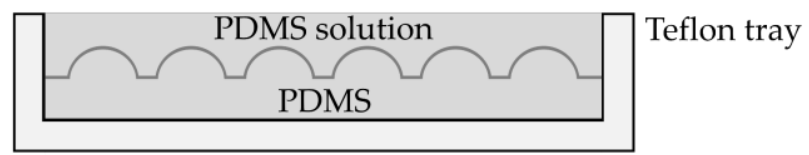

V. Secondary PDMS mould

\section{PDMS}

VI. Hot emboss PVDF

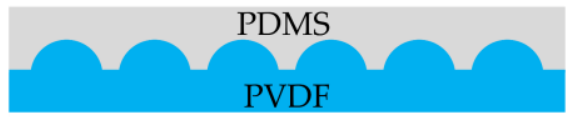

VII. Final mould of PVDF

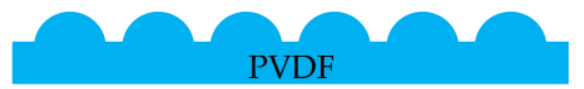

Figure 1. Preparation and morphology of PDMS and PVDF moulds. A schematic of the fabrication of the PDMS moulds and PVDF mould. First, a PDMS solution was cast on a silicon master mould with concave hemispherical microwells situated in a Teflon tray (I) to produce a primary PDMS mould (II). After curing, this PDMS mould was silanised (III), placed in a Teflon tray and a PDMS solution was cast on it (IV). The solution was cured to produce a secondary PDMS mould with concave microstructures (V). This flexible mould could be used to hot emboss (VI) a PVDF mould with convex hemispherical microstructures (VII).

The primary PDMS mould was then placed in a Teflon tray and a PDMS solution (1:10 weight ratio of curing agent to PDMS) was cast over it (Figure 1.IV). It was then left at room temperature for 3 days to cure. Afterwards, a secondary PDMS mould with concave microwells was 
PTMC-microwell membranes for airway epithelial-endothelial cell model

acquired (Figure 1.V and 2). The PVDF mould was fabricated by placing a secondary PDMS mould (Figure $1 . \mathrm{V}$ ) with the concave side of the microwells facing down on top of a PVDF plate (Figure 1.VI) in a hot press (Fontijne THB008, The Netherlands). The press was heated to $170{ }^{\circ} \mathrm{C}$, after which a pressure of 0.12 bar was applied for 10 minutes to hot emboss the PVDF (Figure 1.VI). The press was then cooled passively for 4 hours under pressure to a temperature of approximately $55^{\circ} \mathrm{C}$. The press was then actively cooled to room temperature, after which the PVDF mould was removed from the press. A thin bonding layer of titanium was then applied on the PVDF mould, followed by a stainless-steel coating of approximately $100 \mathrm{~nm}$.
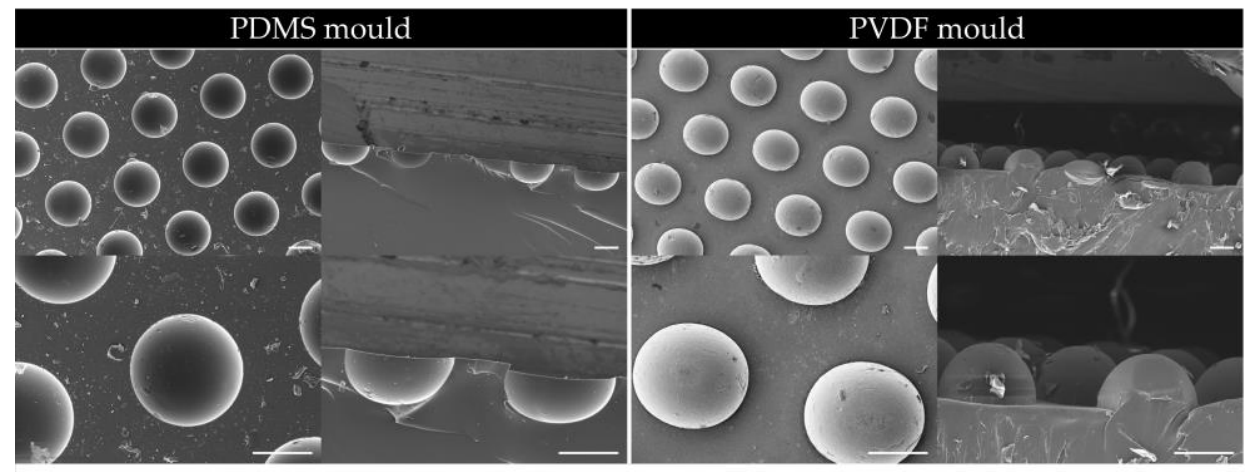

Figure 2. SEM images of the top and cross-section of the secondary PDMS mould and PVDF mould. Magnification of top row: 100x, bottom row: 250x. Scale bar: $100 \mu \mathrm{m}$, applies to all images.

\subsection{Poly(trimethylene carbonate) (PTMC) synthesis and membrane fabrication}

Previously, we have described the PTMC synthesis and fabrication of flat membranes [13]. PTMC synthesis: Briefly, PTMC synthesis involved ring-opening polymerisation of 1,3-trimethylene carbonate (TMC) after which the PTMC was purified and dried. Polymer dope composition: Polymer dopes for the fabrication of the PTMC membranes were prepared as before [13] and consisted of $600 \mathrm{~kg} / \mathrm{mol}$ PTMC, poly(ethylene oxide) (PEO), pentaerythritol triacrylate (PETA), Irgacure 2959 and 
PTMC-microwell membranes for airway epithelial-endothelial cell model

hexanol dissolved in chloroform. Hexanol was added in a 1:1 and 1:3 weight ratio of PTMC to hexanol and served as a non-solvent. Membrane fabrication for flat membranes: The polymer dopes were cast in $500 \mu \mathrm{m}$ thick layers on silicon wafers. EIPS was then performed to form the membranes, after which the membranes were photo-crosslinked using UV-light, and then washed and dried. Based on the 1:1 and 1:3 weight ratio of PTMC to hexanol, the flat membranes were referred to as M1 and M3, respectively [13]. Membrane fabrication for $\mu \mathrm{W}$ membranes: Polymer dopes with the same composition as those for the flat M1 and M3 membranes were used to prepare two types of $\mu \mathrm{W}$ membranes. To prepare the $\mu \mathrm{W}$ membranes, the polymer dopes were cast on the microstructured PVDF moulds in $700 \mu \mathrm{m}$ thick layers, i.e. $700 \mu \mathrm{m}$ above the microstructures of the mould or approximately $800 \mu \mathrm{m}$ thick from the base of the mould. EIPS was then performed in the same manner as for the flat membranes [13]. After EIPS and prior to photo-crosslinking, $\mu \mathrm{W}$ membranes were carefully taken off the moulds and placed on silicon wafers and then photo-crosslinked. The crosslinking and subsequent washing, drying and storage of the $\mu \mathrm{W}$ membranes was the same as for the flat membranes [13].

\section{$2.4 \mathrm{Gel}$ content of the membranes}

Gel content was determined as described previously [13]. Briefly, samples of membranes $(\mathrm{N} \geq 6)$ were cut and weighed (MBefore). Samples were then submerged in an excess of chloroform for two days, dried and weighed again (MAfter). Gel content was then determined with Equation 1.

$$
\text { Gel content }=\frac{\mathrm{M}_{\text {After }}}{\mathrm{M}_{\text {Before }}} \cdot 100 \%
$$

\subsection{Membrane morphology, pore diameter and thickness}

Scanning electron microscopy (SEM) was used to assess the membrane morphology. Samples were cut from dry membranes. For the crosssection of membranes, the samples were dipped in liquid nitrogen and then broken. Samples were gold-sputtered and imaged using a JSM- 
PTMC-microwell membranes for airway epithelial-endothelial cell model

6010LA from JEOL (Tokyo, Japan) at $5 \mathrm{kV}$. Thickness, pore diameter and microstructure dimensions were determined by analysing images of membranes cast on different days using ImageJ software. To determine the pore diameter of membranes, the average pore diameter was calculated per pore. Average thickness, pore diameter and microstructure dimensions were then calculated per sample, after which total averages for all samples (i.e. thickness $(\mathrm{N} \geq 6)$, pore diameter $(\mathrm{N} \geq 3)$, microstructure dimensions $(\mathrm{N} \geq 3))$ were determined.

\subsection{Membrane mounting and coating in inserts and chips}

Samples of M1 and $\mu \mathrm{W}$ membranes were mounted in cell culture inserts, sterilised and coated as described before [14]. Mounting PTMC membranes in cell culture inserts and chips: Briefly, circular samples with a diameter of $15 \mathrm{~mm}$ were punched and secured to the underside of cell culture inserts using poly(ether ether ketone) (PEEK) rings. The membranes and inserts were then placed in Falcon ${ }^{\circledR}$ 12-well companion plates with the upper rim of the inserts on top of thin rings to raise the inserts and allow cell culture medium to reach the underside of the inserts. Chips from Micronit Microtechnologies were used for the chip experiments. These chips consist of three layers: a bottom layer which acted as the blood compartment and harnessed the LMVECs, a top layer which formed the top air compartment where Calu-3 cells were grown and lastly, a middle layer which, together with a porous membrane, separated the top and bottom compartment. In this study, special middle layers of chips lacking a porous membrane were used. Instead, an acrylicbased adhesive layer was present to allow a custom membrane to be applied to the chip layer. In this study, samples of the $\mu \mathrm{W}$ membranes were applied to the underside of the middle layer of the chips using this adhesive layer (Figure 3A). In the case of the $\mu \mathrm{W}$ membranes, the concave side of the microwells was always facing the top of the inserts and chips. Sterilisation of membranes, inserts and chips: The assembled inserts with PTMC membranes and control inserts with PET membranes were submerged in $70 \%$ ethanol for at least 60 minutes, after which they were left to dry. The chips with $\mu \mathrm{W}$ membranes were submerged for 20 
PTMC-microwell membranes for airway epithelial-endothelial cell model

minutes in $70 \%$ ethanol since the adhesion layer between the membrane and chip was somewhat sensitive to ethanol. The assembled inserts and chips were then washed and kept in Dulbecco's PBS without $\mathrm{Ca}^{2+} / \mathrm{Mg}^{2+}$ (DPBS).

Coating of membranes in inserts: After sterilisation, a L-DOPA coating followed by a fibronectin, collagen I and bovine serum albumin (FN/Col I/BSA) coating was applied to the membranes, according to a previous study [14]. Briefly, L-DOPA was dissolved in Tris buffer for 45 minutes at $37^{\circ} \mathrm{C}$. Afterwards, the membrane samples were incubated in L-DOPA solution for 20 hours in an incubator at $37^{\circ} \mathrm{C}$ after which the membranes were clearly coated (Figure 3B). The samples were then washed and kept in DPBS in a cell culture incubator. We noticed that the $\mu \mathrm{W}$ membranes would sometimes partially detach from the chips. This could be solved by placing the membrane correctly onto the adhesive layer while wet, waiting for 10-15 minutes as to let the membrane and chip dry somewhat and then applying some force to re-attach the loose part of the membrane to the chip. After this, problems with adhesion of the membranes to the chip did not occur throughout the entire experiments. Probably, uncrosslinked end-groups of the L-DOPA stimulated the adhesion of the membranes to the adhesive layer. Then a solution of FN/Col I/BSA was added to samples and incubated for one hour after which the samples were washed with DPBS and kept in DPBS in an incubator at $37^{\circ} \mathrm{C}$.

\subsection{Cell culture}

\subsubsection{Cell culture media}

Calu-3 medium: Cell culture medium for the Calu-3 cells contained 88 vol\% Eagle's Minimum Essential Medium (EMEM), 10 vol\% fetal bovine serum (FBS), $1 \mathrm{vol} \%$ penicillin/streptomycin (P/S) and $1 \mathrm{vol} \%$ Glutamax $^{\mathrm{TM}}$. LMVEC medium: For the culturing of LMVECs, a 'microvascular endothelial cell growth medium kit enhanced' was used according to the manufacturer's instructions. 1:1 medium: The cell culture medium for the co-cultures comprised $50 \mathrm{vol} \%$ Calu-3 and $50 \mathrm{vol} \%$ LMVEC medium. 
PTMC-microwell membranes for airway epithelial-endothelial cell model

\subsubsection{Cells and general cell culture}

Calu-3 cells: Calu-3 cells (passage 8) were obtained from The Department of Instructive Biomaterials Engineering, Maastricht University, Maastricht, The Netherlands as previously described [14]. Cells were cultured in culture flasks in Calu-3 cell medium and split at 70\% confluence by trypsinisation. Cells used for experiments in inserts and chips were between passage 11 and 16. LMVECs: LMVECs were purchased from Pelobiotech GmbH, Planegg/Martinsried, Germany (cat. ACBRI 468) and cultured in LMVEC medium in $0.1 \%$ gelatin-coated culture flasks. When confluent, the cells were split once by trypsinisation and later used in inserts and chips at passage 8. All cells were cultured at $5 \% \mathrm{CO}_{2}$ and $37^{\circ} \mathrm{C}$ in a humid environment. The cell culture medium was replaced three times per week.

\subsubsection{Cell seeding and culturing on inserts and chips}

Cell culturing in cell culture inserts: Cell culture experiments of Calu-3 cell and LMVEC co-cultures on coated $\mu \mathrm{W}, \mathrm{M} 1$ and PET membranes in inserts were performed as described previously [14]. Briefly, LMVECs were seeded on the underside of coated $\mu \mathrm{W}, \mathrm{M} 1$ and PET membranes in inserts at a density of 80,000 cells $/ \mathrm{cm}^{2}$ (Scheme 1A, day 0) by flipping the cell culture inserts upside down. After attachment of the LMVECs, inserts were submerged in LMVEC medium in their correct orientation. The next day (day 1), Calu-3 cells were seeded in Calu-3 medium on the top of the membranes at 120,000 cells $/ \mathrm{cm}^{2}$. On day 2, cultures were switched to 1:1 medium. Samples were then cultured submerged until day 9 , after which the Calu-3 cells were transferred to air-liquid-interface (ALI). The medium was refreshed three times a week. Electrical resistance and FITCdextran assays were executed on day 22 and 23, respectively (13 and 14 days at ALI, respectively) (see sections 2.7.4 and 2.7.5). Finally, the cells were fixed with $4 \%$ paraformaldehyde (PFA) or used for live/dead staining on day 24 (day 15 at ALI) (see Section 2.7.7). 


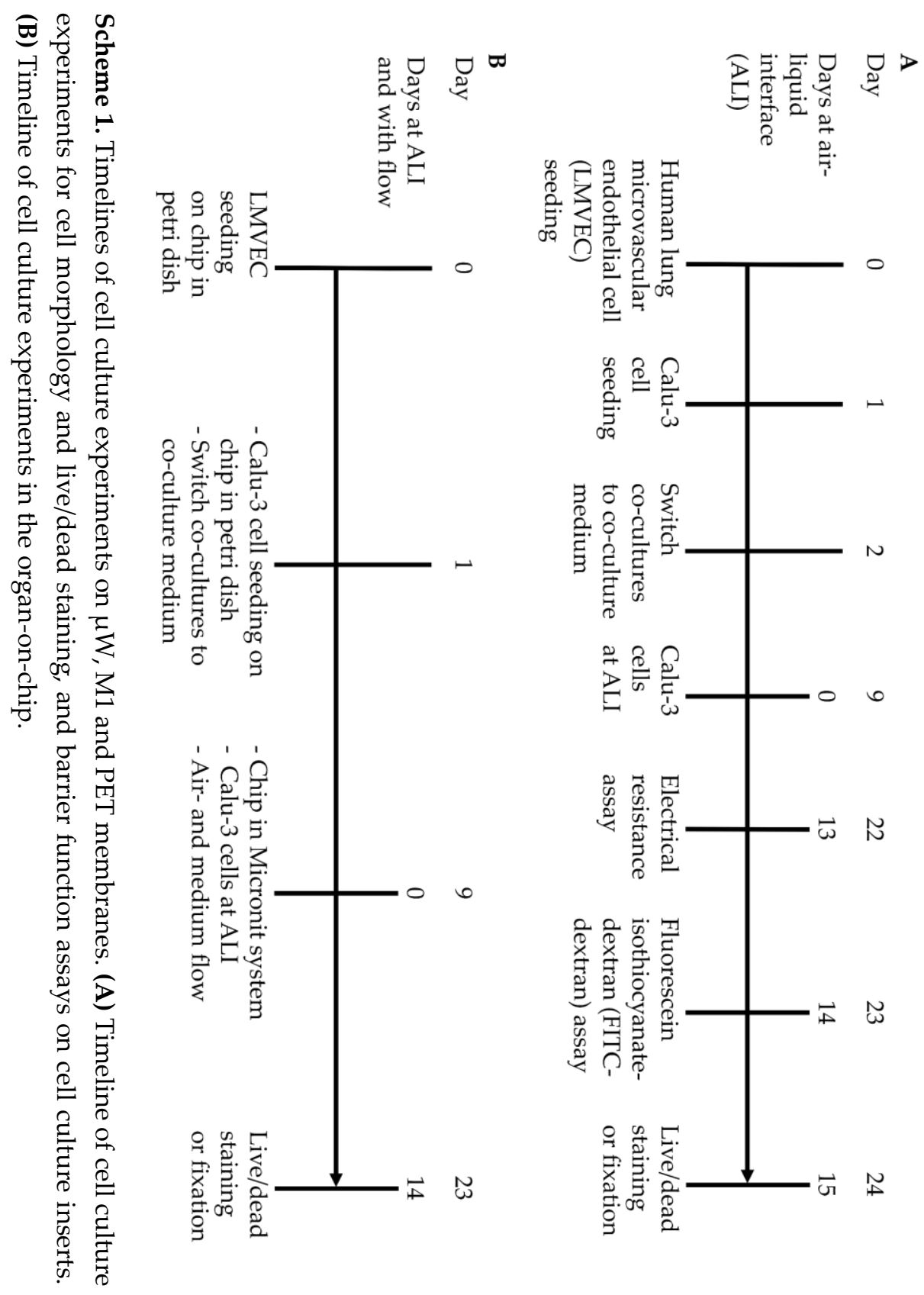


PTMC-microwell membranes for airway epithelial-endothelial cell model

Cell culturing in chips: Cell culturing in chips was largely like that of the experiments in cell culture inserts. Chips with coated $\mu \mathrm{W}$ membranes were flipped upside down (i.e. with the convex side of the microwells facing up) and suspended on O-rings in a petri dish. LMVECs were then seeded on the convex side of the microwells at a density of 80,000 cells $/ \mathrm{cm}^{2}$ (Scheme 1B). To avoid dehydration of the cells, cell culture medium was added under the chip. Due to adhesion and cohesion of the medium and hydrophobicity of the petri dish, the medium remained underneath the chip. After attachment of the cells, i.e. approximately 2 hours, the chip was rinsed with fresh medium and suspended on O-rings in a new petri dish where it was submerged in LMVEC medium overnight. On day 1 (Scheme 1B), the chip was flipped and placed in a new petri dish on top of O-rings. Medium was again added underneath the chip to avoid dehydration of the LMVECs. Calu-3 cells were then seeded on the top of the membranes in the chips at 120,000 cells $/ \mathrm{cm}^{2}$ in Calu-3 medium. After attachment of most cells, approximately 4 hours after seeding, the cocultures were switched to 1:1 medium and kept like this for 8 days to grow the cells to confluency. On day 9, the chips including cells were placed into the chip holder (Figure 3C). Cell culture medium (i.e. 1:1 medium) flow through the bottom compartment of the chip holding the LMVECs was then initiated $(0.7 \mu \mathrm{l} / \mathrm{s})$ by actively pumping the medium out of the chip via a medium outlet, through a medium reservoir and back into the chip (Figure 3C-E). The top compartment, holding the Calu-3 cells, was set to ALI and airflow $(1.3 \mu \mathrm{l} / \mathrm{s})$ through this compartment was initiated by actively pumping the air out of the chip via an air outlet. The air then passed through an air reservoir that had access to fresh air via a $0.2 \mu \mathrm{m}$ filter and then flowed back into the chip via an air inlet (Figure 3C-E). A Watson-Marlow SciQ 323U pump and a Pharmacia P-1 Peristaltic Pump were used to establish the air- and blood flow, respectively. The Calu-3 cells were transferred to ALI on day 9. The chip was then run for 14 days, after which the air- and blood flow were stopped and the chip was taken out of the holder. Cells on the chip were then fixed or used for a live/dead staining as described in Section 2.7.7. 


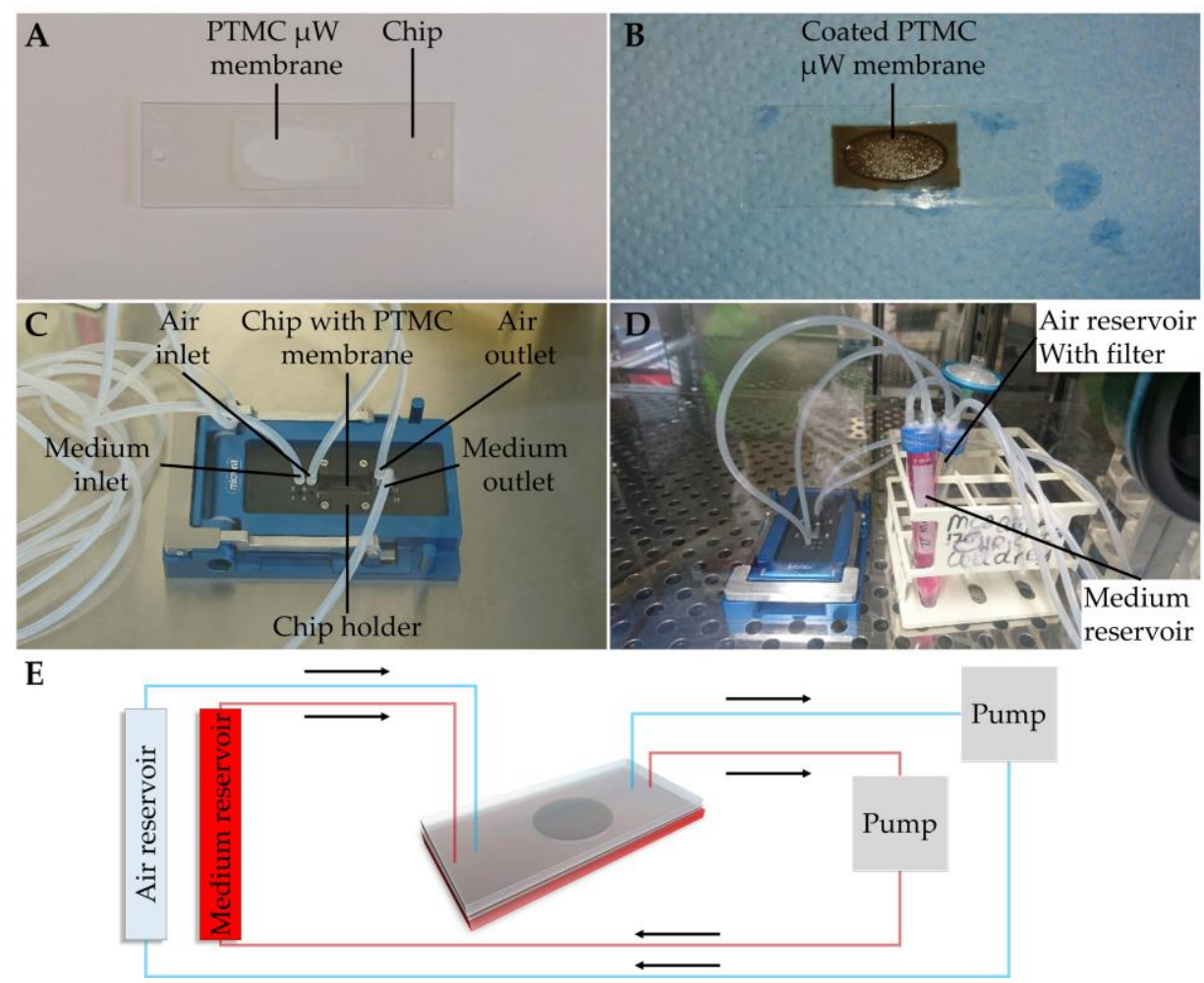

Figure 3. Chip setup with $\mu \mathrm{W}$ membranes. (A,B) Shown is a $\mu \mathrm{W}$ membrane glued to the middle layer of an organ-on-chip (A) after application and (B) after coating with L-DOPA. (C-E) A $\mu \mathrm{W}$ membrane with a Calu-3 cell and LMVEC co-culture in the chip setup. (C) After cells grew confluent, the PTMC membrane and coculture were put in the chip holder. (C,E) Air and cell culture medium then entered the top and bottom compartment of the chip, respectively, through dedicated air and medium inlets and left the chip via an air or medium outlet, respectively. (D,E) The chip was supplied with fresh air and medium via an air reservoir with a filter which was in contact with the air in the incubator and a medium reservoir, respectively. The air and medium were actively pumped out of the chip and back in to their respective reservoirs. The closed nature of the system caused a continuous circulation of air and medium through the chip. 
PTMC-microwell membranes for airway epithelial-endothelial cell model

\subsubsection{Electrical resistance}

Electrical resistance measurements were done as described earlier [14]. Briefly, samples of $\mu \mathrm{W}, \mathrm{M} 1$ and PET membranes in inserts, without and with co-cultures on them, were placed in a cellZscope system (nanoAnalytics $\mathrm{GmbH}$, Münster, Germany) and electrical resistance of the samples was measured for at least one hour with cellZscope software (version 2.2.1 from nanoAnalytics). Data of the membranes without cells was collected from samples of one experiment $(\mathrm{N}=4)$, while those of the membranes with co-cultures originated from two experiments ( $\mathrm{N} \geq 5$ for each experiment). Other settings of the system were the same as described before [14]. Data of the $\mu \mathrm{W}$ samples were adjusted for their increased surface area.

\subsubsection{Fluorescein isothiocyanate-dextran (FITC-dextran) assay for apparent permeability}

Apparent permeability ( $\mathrm{P}_{\text {app }}$ ) of FITC-dextran assay was determined as previously described [14]. A solution of $4 \mathrm{~kg} / \mathrm{mol} \mathrm{FITC-dextran}(1 \mathrm{mg} / \mathrm{mL})$ was added to the top of cell culture inserts with $\mu \mathrm{W}, \mathrm{M} 1$ and PET membranes, both without and with co-cultures. Fresh medium was added underneath these inserts. After 60 minutes in an incubator, samples of the medium underneath the membranes were collected and the inserts and cells were washed with medium and kept in fresh medium. Analysis of the medium samples (in triplicate), pure medium and a concentration series of FITC-dextran solutions was done in a Tecan Infinite M200 Pro® plate reader with $490 \mathrm{~nm}$ and $520 \mathrm{~nm}$ as the excitation and emission wavelengths, respectively. Data of the bare membranes originated from one experiment $(\mathrm{N}=4)$, data of the co-cultures originated from two separate experiments ( $\mathrm{N} \geq 6$ for each experiment). The Papp was determined the same way as described before [14]. Data of the $\mu \mathrm{W}$ samples was corrected for their larger surface area compared to the M1 and PET membranes. 
PTMC-microwell membranes for airway epithelial-endothelial cell model

\subsubsection{Statistical analysis}

For statistical analysis of the gel content, a student T-test was performed with $\mathrm{P}<0.05$. Analysis of all other data was done using a one-way ANOVA and a Bonferroni post-hoc test with $\mathrm{P}<0.05$. All analyses were done in Graphpad Prism 5 software.

\subsubsection{Immunocytochemistry}

Samples of co-cultures in both inserts and chips were either fixed and stained for ZO-1/CD31 or used in a live/dead staining to assess cell viability. For both assays, chip experiments were stopped, and the chip was suspended on O-rings in a petri dish to allow access of, for example, medium and PFA to the underside of the chip. Fixation: To fix the cells in the inserts and chips, samples were washed once with $37{ }^{\circ} \mathrm{C}$ DPBS followed by incubation in sterile $4 \mathrm{vol} \%$ PFA of $37^{\circ} \mathrm{C}$ for 45 minutes at 20 ${ }^{\circ} \mathrm{C}$. Samples were then washed with DPBS and stored at $4{ }^{\circ} \mathrm{C}$ in DPBS. ZO-1 and CD31 staining: Staining of ZO-1 and CD31 was executed as described before [14]. Briefly, samples were incubated with a $0.1 \%$ Triton $^{\mathrm{TM}}$ X-100 solution followed by blocking with a solution of BSA, FBS and Tween ${ }^{\circledR 2} 20$ and an incubation with ZO- 1 antibody overnight at $4{ }^{\circ} \mathrm{C}$. Afterwards, samples were incubated with Alexa fluor Goat $\alpha$ Mouse 488 antibody followed by an overnight incubation with a CD31 antibody at 4 ${ }^{\circ} \mathrm{C}$. Subsequently, samples were incubated with an Alexa fluor Donkey $\alpha$ Rabbit 568 antibody followed by an incubation with a DAPI solution. All incubation steps were performed in the dark and samples were washed with DPBS between steps. For imaging, samples were mounted on microscopy slides together with mounting medium. Live/dead staining for cell viability: A live/dead staining was performed to assess the viability of the cultures as described before [14]. Samples were washed once with cell culture medium and then incubated with a solution of cell culture medium containing calcein, ethidium homodimer I and Hoechst. Afterwards, samples were placed on microscopy slides and imaged. Imaging: A Nikon Eclipse 400 microscope (Nikon, Tokyo, Japan) with Nikon NIS-Elements Imaging software (version 4.51.01) was used to image all samples. 
PTMC-microwell membranes for airway epithelial-endothelial cell model

\section{Results and discussion}

\subsection{Fabrication of flat and microwell membranes}

In this study, we developed flat (M1) and microwell $(\mu W)$ PTMC membranes with pores using EIPS, photo-crosslinking [13] and micromoulding. Presence of hexanol in the polymer solution was necessary for EIPS and pore formation. Initially, polymer solutions with PTMC to hexanol ratios of 1:1 (w/w) and 1:3 (w/w) were used, similar to those used for preparation of flat M1 and M3 PTMC membranes in a previous study [13]. For $\mu \mathrm{W}$ membranes, the solutions were cast on moulds with convex hemispherical microwells. Casting $500 \mu \mathrm{m}$ thick polymer layers on top of the microstructures of the moulds yielded membranes with pinholes (data not shown). Casting $700 \mu \mathrm{m}$ thick polymer solution layers (i.e. $700 \mu \mathrm{m}$ above the microstructures) still resulted in defects in membranes with the 1:3 (w/w) PTMC to hexanol ratio, but avoided defects in the membranes with a 1:1 $(\mathrm{w} / \mathrm{w})$ ratio (data not shown). Thus, we continued development and characterisation of the $\mu \mathrm{W}$ membranes with only the 1:1 (w/w) PTMC to hexanol ratio and compared them to flat M1 membranes.

\subsection{Morphology of $\mu W$ and M1 membranes}

\subsubsection{Electron microscopy}

Figure 4 shows SEM images of the air and substrate side and the crosssection of $\mu \mathrm{W}$ and M1 membranes. Surface morphology of these membranes was very similar, both having a rough air side and smoother substrate side. The rough air side was probably caused by water deposition as a result of the humidity during casting and EIPS [13]. Surface porosity of the air and substrate side of the $\mu \mathrm{W}$ membranes, in general, resembled those of the M1 membranes, although the substrate side of the microwells themselves seemed relatively dense. Probably, the extra thickness between the microwells slowed down phase separation. Moreover, during chloroform evaporation, but prior to complete solidification of the membrane, some of the polymer solution probably 
flowed in between the microwells. This resulted in better pore formation in between the microwells than in the microwells themselves.

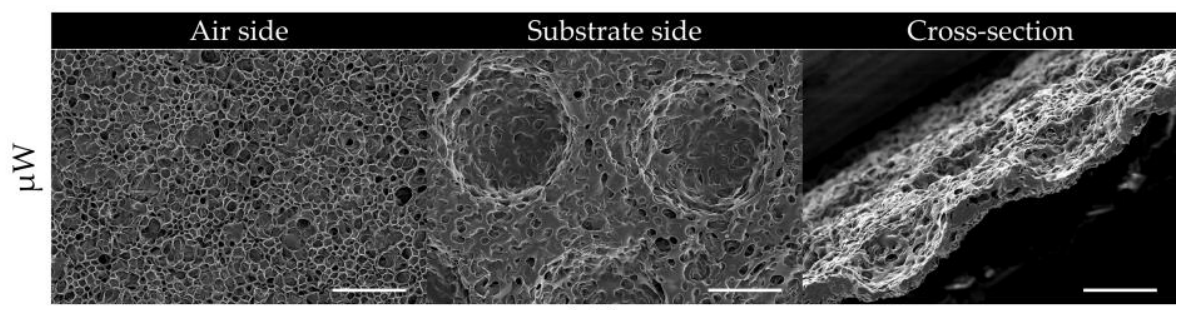

(A)

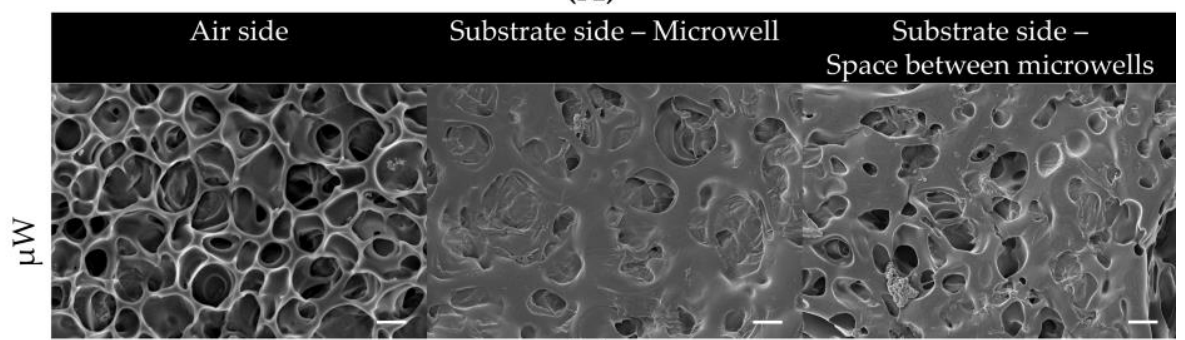

(B)

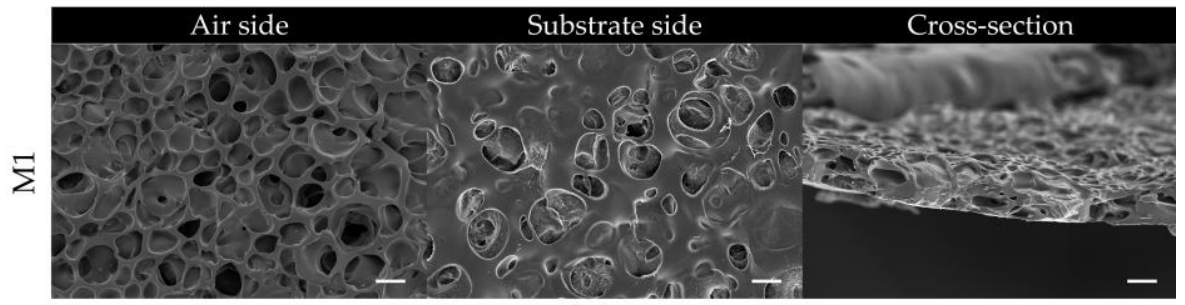

(C)

Figure 4. Morphology of $\mu \mathrm{W}$ membranes. SEM images of PTMC membranes with a 1:1 weight ratio of PTMC and hexanol with microwells $(\mu \mathrm{W})$ or without microwells (M1). Magnification: (A) 250x and (B,C) 1,000x. Scale bar: (A) $100 \mu \mathrm{m}$, $(B, C) 10 \mu \mathrm{m}$. The images are representative of multiple observations $(\mathrm{N} \geq 4)$.

\subsubsection{Pore diameter}

Table 1 shows the surface and cross-sectional pore diameter of $\mu \mathrm{W}$ and M1 membranes. The pore diameter was consistent since there were neither significant differences between any of the sides of $\mu \mathrm{W}$ or M1 membranes nor between $\mu \mathrm{W}$ and M1 membranes. 
PTMC-microwell membranes for airway epithelial-endothelial cell model

\subsubsection{Membrane thickness}

Figure 4 and Table 1 display the cross-section and thickness, respectively, of the $\mu \mathrm{W}$ and M1 membranes. While the thickness at the base of the microwells of the $\mu \mathrm{W}$ membranes was similar to that of the M1 membranes, $\mu \mathrm{W}$ membranes were significantly thicker between the microwells (Figure 4 and Table 1), probably due to the earlier mentioned flowing of polymer solution in between the microwells. Moreover, the polymer layer for the $\mu \mathrm{W}$ membranes was thicker than that for the M1 membranes.

Table 1. Pore diameter and thickness of $\mu \mathrm{W}$ and M1 membranes. Thickness and pore diameter data were analysed by one-way ANOVA. Significant differences $(\mathrm{P}<0.05)$ between the area between microwells, the microwells or the average of $\mu \mathrm{W}$ membranes or M1 membranes are marked by $(\mathrm{B} \mu \mathrm{W}),(\mu \mathrm{W}),(\mathrm{Av})$ or (M1), respectively. Pore diameter analysis was based on $\geq 3$ images per condition.

\begin{tabular}{|c|c|c|c|c|c|}
\hline & \multicolumn{3}{|c|}{$\mu W$ membranes } & \multirow{2}{*}{ M1 membranes } \\
\hline & & $\begin{array}{c}\text { Microwells } \\
(\mu W)\end{array}$ & $\begin{array}{c}\text { Between } \\
\text { microwells } \\
(B \mu W)\end{array}$ & $\begin{array}{c}\text { Average } \\
\text { (Av) }\end{array}$ & \\
\hline \multirow{3}{*}{$\begin{array}{l}\text { Average por } \\
\text { diameter } \\
(\mu \mathrm{m}) \\
(\mathrm{N} \geq 3)\end{array}$} & Air side & $4.8 \pm 0.9$ & $5.9 \pm 0.4$ & $5.6 \pm 0.6$ & $5.2 \pm 2.2$ \\
\hline & $\begin{array}{l}\text { Substrate } \\
\text { side }\end{array}$ & $5.0 \pm 1.8$ & $4.7 \pm 2.3$ & $4.8 \pm 1.8$ & $6.4 \pm 1.8$ \\
\hline & Cross-section & $4.8 \pm 1.1$ & $4.6 \pm 1.5$ & $4.7 \pm 0.9$ & $7.7 \pm 2.3$ \\
\hline \multicolumn{2}{|c|}{$\begin{array}{c}\text { Thickness }(\mu \mathrm{m}) \\
\qquad(\mathrm{N} \geq 6)\end{array}$} & $\begin{array}{c}22.6 \pm 6.5 \\
(\mathrm{~B} \mu \mathrm{W}, \mathrm{Av})\end{array}$ & $\begin{array}{c}45.8 \pm 8.1 \\
(\mu \mathrm{W}, \mathrm{Av}, \mathrm{M} 1)\end{array}$ & $\begin{array}{l}36.8 \pm 5.3 \\
(\mu \mathrm{W}, \mathrm{B} \mu \mathrm{W}, \mathrm{M} 1)\end{array}$ & $\begin{array}{c}20.3 \pm 3.4 \\
(\mathrm{~B} \mu \mathrm{W}, \mathrm{Av})\end{array}$ \\
\hline
\end{tabular}


PTMC-microwell membranes for airway epithelial-endothelial cell model

\subsubsection{Microwell dimensions}

Figure 4 and Table 2 show the microwells and the microwell dimensions, respectively, of the $\mu \mathrm{W}$ membranes. Microwells were uniform and did not display defects (Figure 4). Importantly, their diameter (i.e. 176.6 \pm 10.6 $\mu \mathrm{m})$ on the substrate side of the membranes (i.e. the concave side of the microwells) (Table 2) resembled that of alveoli [15] and was similar to the diameter of the microstructures of the PVDF mould (i.e. $183.2 \pm 3.6 \mu \mathrm{m}$ ). The depth of the microwells was different between the substrate and air side of the $\mu \mathrm{W}$ membranes due to the thickness of the membranes between the microwells (Figure 4, Table 2). Also, the depth of the microwells on the $\mu \mathrm{W}$ membranes was lower than the height of the microwells of the PVDF mould (i.e. $41.9 \pm 9.5 \mu \mathrm{m}$ and $97.4 \pm 6.3 \mu \mathrm{m}$, respectively). Thus, instead of hemispherical, the microwells had a less pronounced curvature. Loss of microwell depth during EIPS is unlikely since it would have resulted in partial detachment of the membrane from the mould or defects in the microwells, which were both absent. The $\mu \mathrm{W}$ membranes were removed from the mould prior to crosslinking, since this was difficult to do afterwards. Probably, after removing the $\mu \mathrm{W}$ membranes from the mould, residual chloroform in the membranes evaporated prior to and during photo-crosslinking, decreasing the height of the membranes. Moreover, some creep may have occurred before the membranes were sufficiently crosslinked.

Table 2. Dimensions of microwells of $\mu \mathrm{W}$ membranes. The table shows the depth and diameter of microwells on the substrate and air side of the $\mu \mathrm{W}$ membranes. Data were analysed by T-test. Significant differences $(\mathrm{P}<0.05)$ between the substrate and air side of the PTMC membranes are marked by *. Analysis was based on $\geq 5$ images per condition.

\begin{tabular}{ccc}
\hline Microwells & Substrate side & Air side \\
\hline $\begin{array}{c}\text { Diameter }(\mu \mathrm{m}) \\
(\mathrm{N} \geq 5)\end{array}$ & $176.6 \pm 10.6$ & $172.2 \pm 13.5$ \\
\hline $\begin{array}{c}\text { Depth }(\mu \mathrm{m}) \\
(\mathrm{N} \geq 11)\end{array}$ & $41.9 \pm 9.5^{*}$ & $25.0 \pm 5.9^{*}$ \\
\hline
\end{tabular}


PTMC-microwell membranes for airway epithelial-endothelial cell model

\subsection{Gel content}

The gel content of the $\mu \mathrm{W}$ membranes was significantly lower than that of the M1 membranes, i.e. $68.8 \% \pm 3.4 \%$ and $79.2 \% \pm 7.3 \%(\mathrm{~N} \geq 6)$, respectively. As described in an earlier study [13], crosslinking agents are probably partially dissolved in residual chloroform and the hexanol during crosslinking. Probably, the amount of residual chloroform in $\mu \mathrm{W}$ membranes mentioned earlier (Section 3.2.4) was relatively high in the thicker $\mu \mathrm{W}$ membranes compared to the M1 membranes, which led to less efficient crosslinking in the former [13]. Nevertheless, the gel content of the $\mu \mathrm{W}$ membranes was within the range of gel content we attained with mechanically stable M0-M3 membranes in a previous study [13]. Besides, $\mu \mathrm{W}$ membranes could be handled well in DPBS and cell culture media, did not swell significantly in these liquids and were not sticky (data not shown).

\subsection{Electrical resistance and apparent permeability of membranes and co-cultures in cell culture inserts}

Figure 5 shows the electrical resistance and Papp of FITC-dextran of $\mu \mathrm{W}$, M1 and PET membranes without cells and with Calu-3 cell and LMVEC co-cultures. $\mu \mathrm{W}, \mathrm{M} 1$ and PET membranes without cells had low electrical resistance, i.e. 14.1, 9.4 and $6.4 \Omega \cdot \mathrm{cm}^{2}$, respectively, and relatively high

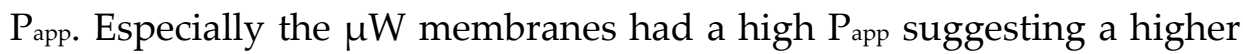
pore interconnectivity in these membranes compared to the M1 membranes.

Co-cultures of cells had higher electrical resistance on all membranes and lower Papp on $\mu \mathrm{W}$ and PET membranes than respective membranes without cells. Statistical analysis without the $\mu \mathrm{W}$ groups showed that cocultures on M1 membranes had significantly lower Papp compared to M1 membranes without cells. The electrical resistance and $P_{\text {app }}$ data suggest that co-cultures created functional barriers on all membranes. 


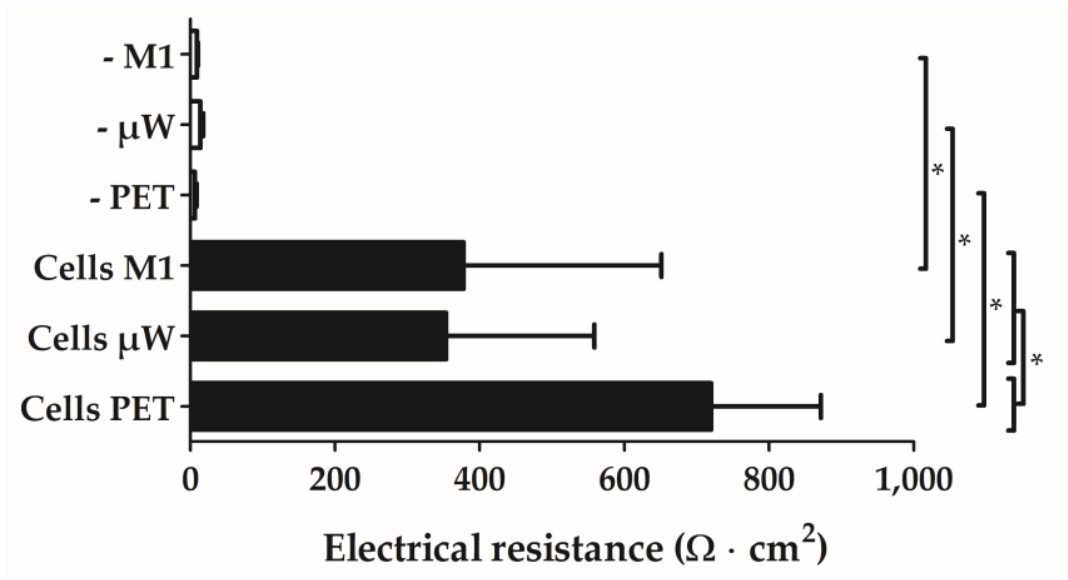

(A)

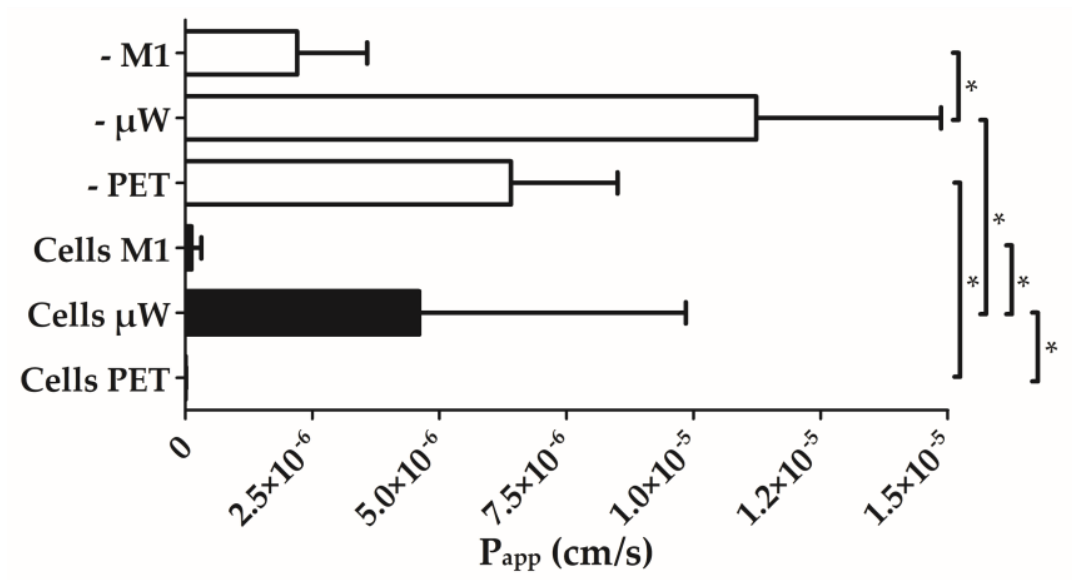

(B)

Figure 5. Electrical resistance and apparent permeability ( $\left.\mathrm{P}_{\text {app }}\right)$ of FITC-dextran across co-cultures of Calu- 3 cells and LMVECs on $\mu \mathrm{W}$, M1 and PET membranes. Calu-3 cells were cultured submerged for 8 days, followed by 2 weeks at ALI. (A) Electrical resistance and (B) FITC-dextran permeability assays were performed on inserts with $\mu \mathrm{W}, \mathrm{M} 1$ or PET membranes containing both Calu-3 cells and LMVECs. Capped lines with asterisks depict significant differences, $\mathrm{P}<0.05 . \mathrm{N} \geq$ 12 for samples with cells, divided over 2 experiments. $\mathrm{N}=4$ for samples without cells, originating from 1 experiment.

Co-cultures on PET membranes had higher electrical resistance than the other co-cultures. Co-cultures on M1 and PET membranes had lower Papp than co-cultures on $\mu \mathrm{W}$ membranes, probably partially due to the 
PTMC-microwell membranes for airway epithelial-endothelial cell model

relatively high permeability of the bare $\mu \mathrm{W}$ membranes. Nonetheless, the electrical resistances of all co-cultures were well within the range of Calu3 cell barriers [14,16-20], but were quite low compared to other Calu-3 cocultures $[14,19,20]$. The Papp of the co-cultures on PET and M1 membranes were similar to those of previously studied co-cultures [14].

\subsection{ZO-1, CD31 and live/dead staining of Calu-3 cell and LMVEC co-cultures in cell culture inserts and microfluidic chips}

Figure 6 and 7 show ZO-1 staining of Calu-3 cells and ZO-1/CD31 staining of LMVECs, respectively, of the co-cultures on M1 and PET membranes in cell culture inserts and on $\mu \mathrm{W}$ membranes in both cell culture inserts and the microfluidic organ-on-chip system from Micronit Microtechnologies. ZO-1 is a tight junction protein and CD31 is a common endothelial cell marker. Figure 8 shows a live/dead staining of similar cultures. Both assays were performed to further assess the co-culture on the $\mu \mathrm{W}$ membranes.

\subsubsection{ZO-1 and CD31 staining}

Calu-3 cells had a typical cobblestone phenotype [14,21] and were positive for the tight junction protein ZO-1 on all membranes, both in inserts and the chip (Figure 6). This agrees with the electrical resistance and $P_{\text {app }}$ data since tight junctions are important in establishing an epithelial barrier. Calu-3 cells covered the PET and M1 membranes and seemed to do so as well on $\mu \mathrm{W}$ membranes. There was no distinctive difference in the ZO-1 staining of Calu-3 cells between all samples, including those on the chips.

Figure 6. ZO-1 staining of Calu-3 cells from co-cultures with LMVECs on $\mu \mathrm{W}$ and M1 membranes. Cells grown on inserts with PET membranes were used as controls. Calu-3 cells were cultured submerged for 8 days, followed by 2 weeks of culture at ALI. Cells were stained for the cell nucleus (blue) and ZO-1 (green), magnification: $1^{\text {st }}$ and $3^{\text {rd }}$ column $400 x, 2^{\text {nd }}$ and $4^{\text {th }}$ column $800 x$, scale bar: $100 \mu \mathrm{m}$. Images are representative of observations of multiple samples from two independent experiments $(\mathrm{N}=3)$. In the case of the chip samples, three individual chips were stained $(\mathrm{N}=3)$. 


\section{- Chapter 7 -}

PTMC-microwell membranes for airway epithelial-endothelial cell model

Bottom of well Top of well

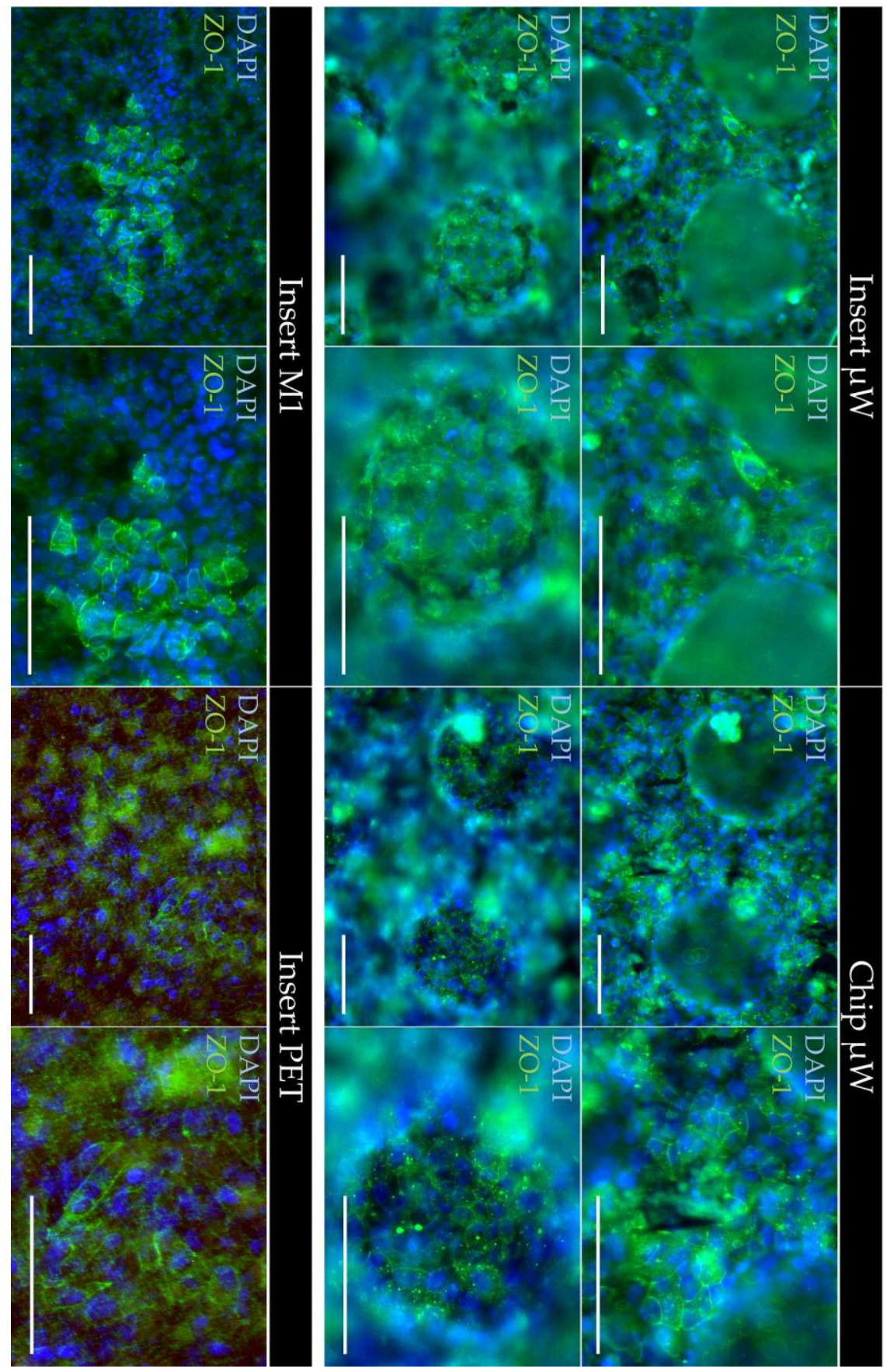


PTMC-microwell membranes for airway epithelial-endothelial cell model

LMVECs were present on PET, M1 and $\mu \mathrm{W}$ membranes, the latter including the chips, and expressed both CD31 and ZO-1 (Figure 7). Although ZO-1 and CD31 are usually localised at the cell membrane, both proteins were distributed throughout the cytoplasm of the LMVECs, which agrees with previous studies [14,22]. It is probably partially caused by the presence of epithelial cells [22]. LMVECs with such diffuse CD31 expression have proven to significantly contribute to the barrier function of the co-culture without showing signs of endothelial-to-mesenchymal transition (EndoMT) (i.e. $\alpha$-SMA expression) [14].
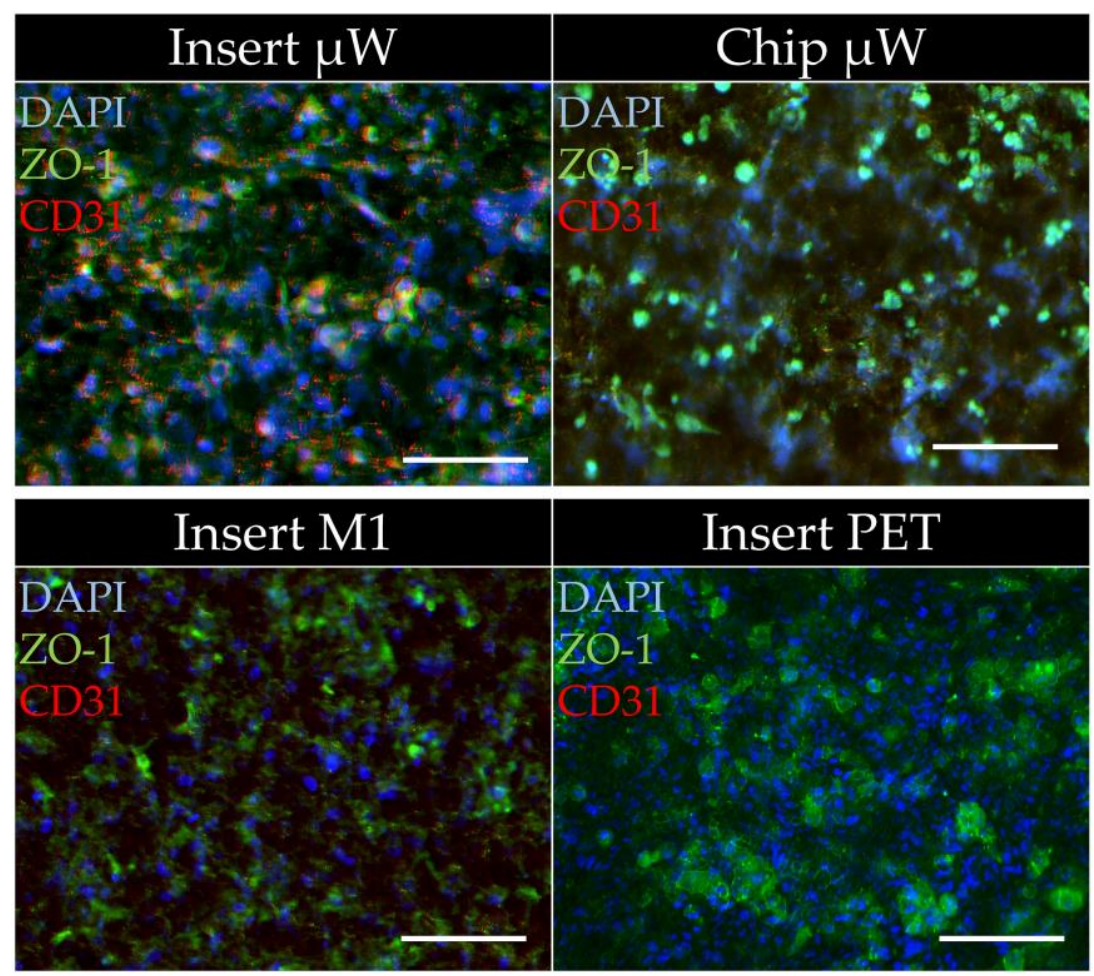

Figure 7. ZO-1 and CD31 staining of LMVECs from co-cultures with Calu-3 cells on $\mu \mathrm{W}$ and M1 membranes. Cells grown on inserts with PET membranes were used as controls. LMVECs were stained for the cell nucleus (blue), ZO-1 (green) and CD31 (red) after 3 weeks of submerged culture. Magnification: 400x, scale bar: $100 \mu \mathrm{m}$. Images are representative of observations of multiple samples from two independent experiments $(\mathrm{N}=3)$. In the case of the chip samples, three individual chips were stained $(\mathrm{N}=3)$. 


\subsubsection{Live/dead staining for cell viability}

All cultures in the inserts showed good viability with little cell death, as the calcein signal was present across the entire samples, while there was only little ethidium homodimer I signal (Figure 8). However, the Calu-3 cells on the $\mu \mathrm{W}$ membranes displayed higher cell death in the chips than in the inserts.

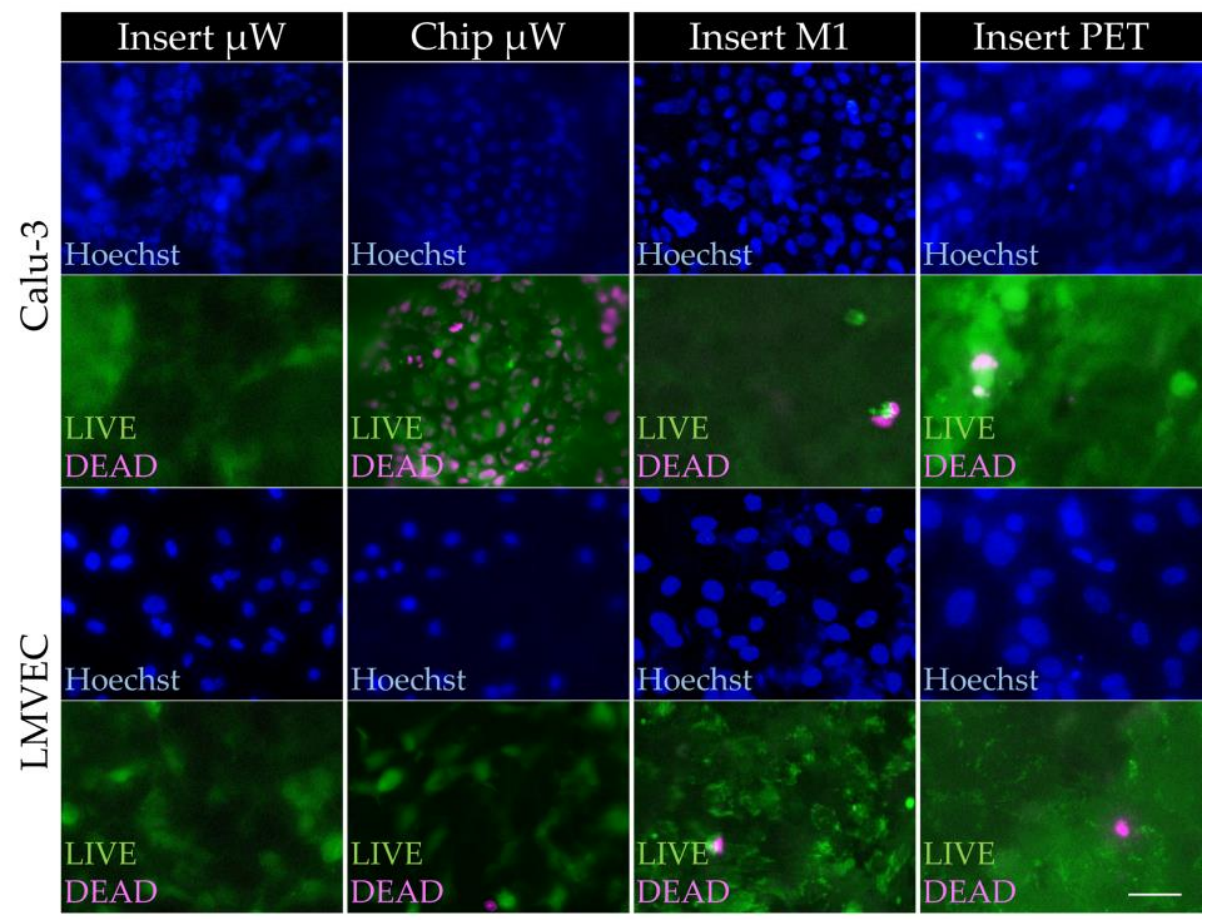

Figure 8. Live/dead staining of co-cultures of Calu-3 cells and LMVECs on $\mu \mathrm{W}$ and M1 membranes. Co-cultures of Calu-3 cells and LMVECs were cultured in inserts on $\mathrm{M} 1$ and $\mu \mathrm{W}$ membranes and in inserts with PET membranes as controls. Moreover, similar co-cultures were cultured on $\mu \mathrm{W}$ membranes in a microfluidic chip system. Calu-3 cells were cultured submerged for 8 days, after which they were cultured at ALI for 2 weeks. LMVECs were cultured submerged throughout the experiment. Shown are the cell nuclei (Hoechst: blue), the live cells (calcein: green) and dead cells (ethidium homodimer I: magenta). Magnification: 800x. Scale bar: $50 \mu \mathrm{m}$, applies to all images. Images are representative of observations of multiple samples from two independent experiments $(\mathrm{N}=3)$. In the case of the chip samples, two individual chips were stained. 
PTMC-microwell membranes for airway epithelial-endothelial cell model

This was probably due to cellular stress caused by the airflow in the chips. There was no such effect of the medium flow on the LMVECs. Moreover, the calcein signal of the Calu-3 cells and LMVECs in the chips did not differ from those in the inserts.

\subsection{Calu-3 cell and LMVEC co-cultures on EIPS/micromoulding- based $\mu W$ membranes in a lung-on-a-chip}

In this study, we combined for the first time EIPS and micromoulding to develop flexible PTMC membranes with hemispherical microwells, which were porous, permeable, and mechanically stable. They were also successfully integrated into cell culture inserts and a microfluidic chip. Overall, microwells were replicated well, but had a less pronounced curvature than was intended.

Calu-3 cells and LMVECs grew well on the membranes. Calu-3 cells seemed to cover the membranes including the microwells, although a Zstack of these samples would have to confirm this. Although the cocultures performed well on the $\mu \mathrm{W}$ membranes, co-cultures on PET membranes showed better barrier function. Despite an overall high permeability of the $\mu \mathrm{W}$ membranes, the base of the microwells was quite dense which may have hampered properties of these co-cultures, most notably their barrier function. A high, more homogeneous porosity across the $\mu \mathrm{W}$ membranes would thus be preferable. A higher hexanol concentration in the polymer solution, e.g. a 1:3 (w/w) PTMC to hexanol ratio, could increase the porosity across the entire membrane. However, large pores and pinholes should be avoided, e.g. by decreasing the concentration or molecular weight of PEO [13,23,24]. Moreover, dichloromethane could be used as a solvent to increase the rate of EIPS, thereby limiting coalescence of hexanol, and thus the pore diameter, especially between the microwells where more coalescence of hexanol takes place.

In the future, a more systematic study is needed to investigate the effects of the microwells on the cells, both in static conditions as well as with airand fluid flow. The dimensions of the microwells on the mould could be adapted to increase the final curvature of the $\mu \mathrm{W}$ membranes by creating 
PTMC-microwell membranes for airway epithelial-endothelial cell model

deeper microwells on the mould. More pronounced curvature of the $\mu \mathrm{W}$ membranes could lead to increased expression of tight-junctional proteins, alignment of the cells and their ECM, and mechanical strength of the cell layers [4]. The latter would also be relevant for mechanical stimulation of the model. Next studies could also investigate the responses of primary small airway and alveolar epithelial cells to the curvature of the microwells in static conditions and with air- and fluid flow, as well as introduce immune cells and fibroblasts to truly mimic the in vivo situation.

\section{Conclusions}

In this study, we developed flexible porous PTMC membranes with hemispherical microwells for the first time. These membranes were developed to better resemble the basement membrane of in vivo lung epithelial-endothelial barriers than current flat membranes in in vitro lung models. Co-cultures of Calu-3 cells and LMVECs on the $\mu \mathrm{W}$ membranes in cell culture inserts were ZO-1 and ZO-1/CD31 positive, respectively, showed good viability and formed a functional barrier. The $\mu \mathrm{W}$ membrane and co-culture could also be placed in a commercial organ-onchip system with air- and fluid flow. There were no differences in ZO-1, CD31 and viability between the chip and insert samples, except that Calu3 cells showed higher cell death on the chips, probably due to stress caused by the airflow.

The $\mu \mathrm{W}$ PTMC membranes can be used in a static in vitro lung epithelial and endothelial cell model as well as a microfluidic organ-on-chip system. However, deeper microwells with more pronounced curvature are probably required to affect the cells, e.g. their tight-junctional protein expression and alignment. The presented method to fabricate $\mu \mathrm{W}$ membranes could serve as a basis for improvement of membranes in these specific types of models and of membranes in in vitro models in general. 


\section{- Chapter 7 -}

PTMC-microwell membranes for airway epithelial-endothelial cell model

\section{References}

1. World Health Organization. The Top 10 Causes of Death. Available online: https:/www.who.int/news-room/fact-sheets/detail/the-top-10-causes-of-death (accessed on 28 December 2020).

2. Stucki, A.O.; Stucki, J.D.; Hall, S.R.; Felder, M.; Mermoud, Y.; Schmid, R.A.; Geiser, T.; Guenat, O.T. A lung-on-a-chip array with an integrated bio-inspired respiration mechanism. Lab Chip. 2015, 15, 1302-1310. DOI: 10.1039/c4lc01252f.

3. Huh, D.; Leslie, D.C.; Matthews, B.D.; Fraser, J.P.; Jurek, S.; Hamilton, G.A.; Thorneloe, K.S.; McAlexander, M.A.; Ingber, D.E. A human disease model of drug toxicity-induced pulmonary edema in a lung-on-a-chip microdevice. Sci. Transl. Med. 2012, 4. DOI: 10.1126/scitranslmed.3004249.

4. Chapter 2 of this thesis.

5. Esch, M.B.; Post, D.J.; Shuler, M.L.; Stokol, T. (2011). Characterization of in vitro endothelial linings grown within microfluidic channels. Tissue engineering. Part A. 2011, 17(23-24), 2965-2971. doi: 10.1089/ten.tea.2010.0371.

6. Hebeiss, I.; Truckenmüller, R.; Giselbrecht, S.; Schepers, U. Novel threedimensional Boyden chamber system for studying transendothelial transport. Lab on a chip. 2012 12(4), 829-834. DOI: 10.1039/c2lc20733h.

7. Broaders, K.E.; Cerchiari, A.E.; Gartner, Z.J. (2015). Coupling between apical tension and basal adhesion allow epithelia to collectively sense and respond to substrate topography over long distances. Integr. Biol. 2015, 7(12), 1611-1621. DOI: $10.1039 /$ c5ib00240k.

8. Baptista, D.; Teixeira, L.M.; Birgani, Z.T.; van Riet, S.; Pasman, T.; Poot, A.; Stamatialis, D.; Rottier, R.J.; Hiemstra, P.S.; Habibović, P.; et al. 3D alveolar in vitro model based on epithelialized biomimetically curved culture membranes. Biomaterials. 2021, 266, 120436. DOI: 10.1016/j.biomaterials.2020.120436.

9. Soscia, D.A.; Sequeira, S.J.; Schramm, R.A.; Jayarathanam, K.; Cantara, S.I.; Larsen, M.; Castracane, J. Salivary gland cell differentiation and organization on micropatterned PLGA nanofiber craters. Biomaterials. 2012, 34(28), 6773-6784. DOI: 10.1016/j.biomaterials.2013.05.061.

10. Papenburg, B.J.; Vogelaar, L.; Bolhuis-Versteeg, L.A.M.; Lammertink, R.G.H.; Stamatialis, D.; Wessling, M. One-Step fabrication of porous micropatterned scaffolds to control cell behavior. Biomaterials. 2007, 28, 1998-2009. DOI: 10.1016/j.biomaterials.2006.12.023.

11. Vogelaar, L.; Lammertink, R.G.H.; Barsema, J.N.; Nijdam, W.; Bolhuis-Versteeg, L.A.M.; van Rijn, C.J.M.; Wessling, M. Phase separation micromolding: A new generic approach for microstructuring various materials. Small. 2005, 1, 645-655. DOI: 10.1002/smll.200400128.

12. Chapter 4 of this thesis.

13. Chapter 5 of this thesis

14. Chapter 6 of this thesis.

15. Ochs, M.; Nyengaard, J.R.; Jung, A.; Knudsen, L.; Voigt, M.; Wahlers, T.; Richter, J.; Gundersen, H.J. The number of alveoli in the human lung. American journal 


\section{- Chapter 7 -}

PTMC-microwell membranes for airway epithelial-endothelial cell model

of respiratory and critical care medicine. 2004, 169(1), 120-124. DOI: 10.1164/rccm.200308-1107OC.

16. Grainger, C.I.; Greenwell, L.L.; Lockley, D.J.; Martin, G.P.; Forbes, B. Culture of Calu-3 cells at the air interface provides a representative model of the airway epithelial barrier. Pharm. Res. 2006, 23, 1482-1490. DOI: 10.1007/s11095-006-02550 .

17. Kreft, M.E.; Jerman, U.D.; Lasič, E.; Hevir-Kene, N.; Rižner, T.L.; Peternel, L.; Kristan, K. The characterization of the human cell line Calu-3 under different culture conditions and its use as an optimized in vitro model to investigate bronchial epithelial function. Eur. J. Pharm. Sci. 2015, 69, 1-9. DOI: 10.1016/j.ejps.2014.12.017.

18. Srinivasan, B.; Kolli, A.R.; Esch, M.B.; Abaci, H.E.; Shuler, M.L.; Hickman, J.J. TEER measurement techniques for in vitro barrier model systems. J. Lab. Autom. 2015, 20, 107-126. DOI: 10.1177/2211068214561025.

19. Zhang, F.; Aquino, G.V.; Dabi, A.; Bruce, E.D. Assessing the translocation of silver nanoparticles using an in vitro co-culture model of human airway barrier. Toxicol. In Vitro. 2019, 56, 1-9. DOI: 10.1016/j.tiv.2018.12.013.

20. Dekali, S.; Gamez, C.; Kortulewski, T.; Blazy, K.; Rat, P.; Lacroix, G. Assessment of an in vitro model of pulmonary barrier to study the translocation of nanoparticles. Toxicol. Rep. 2014, 1, 157-171. DOI: 10.1016/j.toxrep.2014.03.003.

21. Kale, S.L.; Agrawal, K.; Gaur, S.N.; Arora, N. Cockroach protease allergen induces allergic airway inflammation via epithelial cell activation. Sci. Rep. 2017, 7, 42341. DOI: 10.1038/srep42341.

22. Weppler, A.; Rowter, D.; Hermanns, I.; Kirkpatrick, C.J.; Issekutz, A.C. Modulation of endotoxin-induced neutrophil transendothelial migration by alveolar epithelium in a defined bilayer model. Exp. Lung Res. 2006, 32, 455-482. DOI: 10.1080/01902140601059463.

23. Papenburg, B.J.; Schuller-Ravoo, S.; Bolhuis-Versteeg, L.A.M.; Hartsuiker, L.; Grijpma, D.W.; Feijen, J.; Wessling, M.; Stamatialis, D. Designing porosity and topography of poly(1,3-trimethylene carbonate) scaffolds. Acta Biomater. 2009, 5, 3281-3294. DOI: 10.1016/j.actbio.2009.05.017.

24. Pêgo, A.P.; Poot, A.A.; Grijpma, D.W.; Feijen, J. Copolymers of trimethylene carbonate and epsilon-caprolactone for porous nerve guides: Synthesis and properties. Biomater. Sci. Polym. Ed. 2001, 12, 35-53. DOI: $10.1163 / 156856201744434$.

25. Bat, E. Flexible scaffolds based on poly(trimethylene carbonate) networks for cardiac tissue engineering. Ph.D. thesis, University of Twente, Enschede, The Netherlands, May 2010. 
- Chapter 7 -

PTMC-microwell membranes for airway epithelial-endothelial cell model 


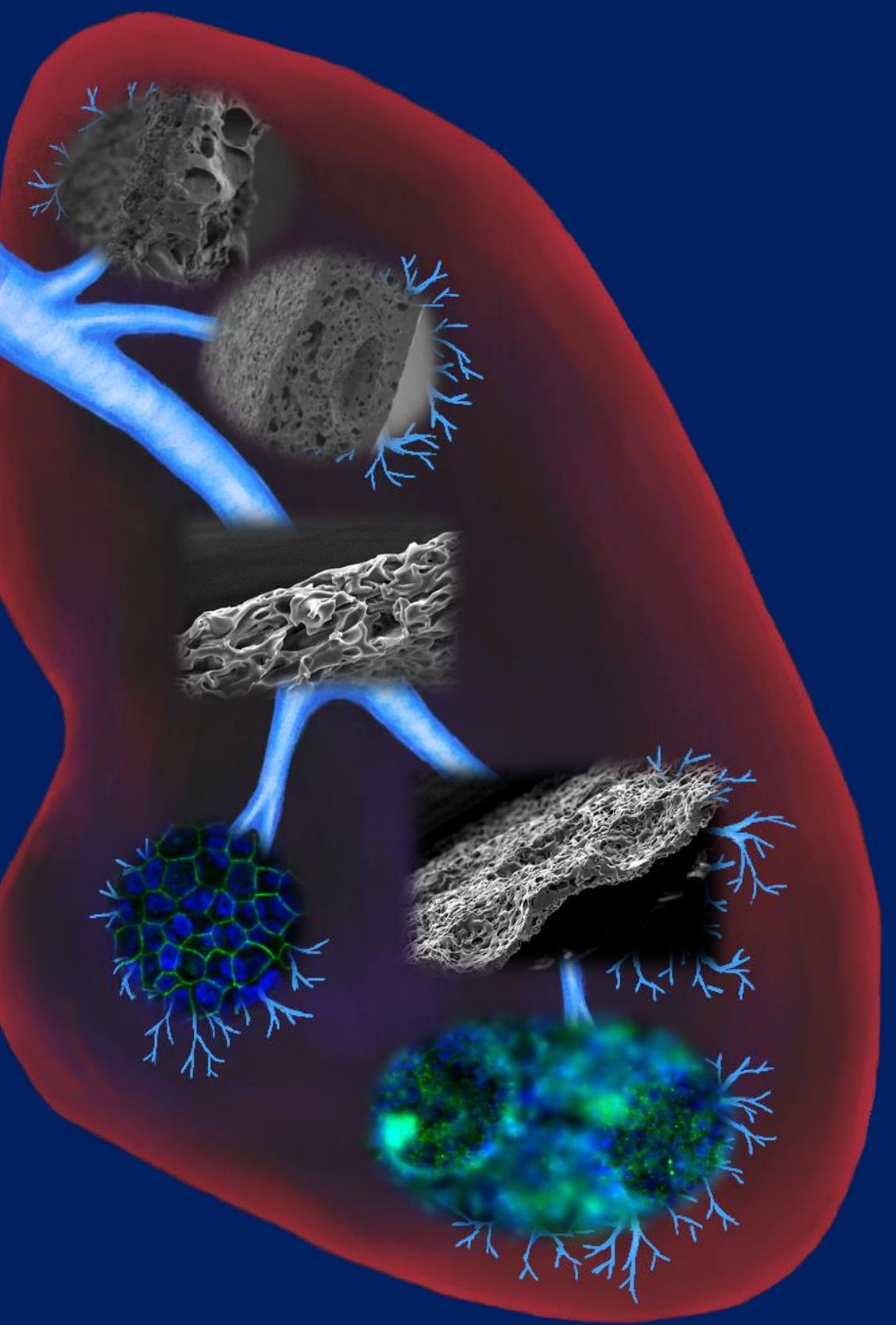




\section{- Chapter 8 -}

\section{Conclusions and future outlook}

Thijs Pasman, Dimitrios Stamatialis and André A. Poot

Technical Medical (TechMed) Centre, Department of Biomaterials Science and Technology, Faculty of Science and Technology, University of Twente, 7522 NB Enschede, The Netherlands 
The exchange of oxygen and carbon dioxide in the alveoli of the lungs is fundamental for the metabolism of our cells and functioning of our bodies. Thus, afflictions to the lungs caused by external factors such as pathogens, genetic defects or other causes can lead to life-threatening disorders such as cystic fibrosis and COPD. As a result, lung diseases are among the most common causes of death [1]. Moreover, with the current pandemic of the SARS-CoV-2 virus, there is an unprecedented interest in lung research and biomimetic models which can provide reliable results for development of drugs and treatments. Unfortunately, traditional models, such as those in culture plates and Transwell@ inserts, lack many of the features of the lung, which limits their reliability. Animal models have the necessary complexity, but there is a growing resistance to using them because of ethical issues and low predictability of the efficacy of drugs and treatments in humans, caused by physiological differences between humans and the animal models. In the case of lung research, LOCs attempt to include the necessary complexity of the lungs while avoiding the ethical issues associated with animal models [2,3]. Moreover, they have the benefits of in vitro models, such as reduced costs and increased control over the study conditions, such as fluid flow and airflow across the cells. However, in many organ models, including LOCs and lung models in general, there is little attention for the membranes on which the cells grow, while there is compelling evidence for the influence of their properties on the results of the models [2].

In Chapter 2, an overview of the state of the art in membranes of OOCs is provided. Disadvantages of traditional models are given, and the need for biomimetic models, i.e. OOCs, is explained. We provide an overview of material properties that influence cell behaviour (e.g. proliferation and differentiation) to indicate the relevance of considering properties of the membranes when developing biomimetic models. Most membranes used in organ models such as OOCs are either made from stiff materials (e.g. PET and PC) with mechanical properties that do not resemble that of the targeted tissue [2,4-6] or materials with other disadvantages, such as low cytocompatibility, e.g. PDMS [2]. Moreover, membranes in OOCs are 
mostly flat, lacking three-dimensional topography that resembles the architecture of a tissue, e.g. the spherical structure of the alveoli. However, application of cells on surfaces with microstructures has proven to influence cell behaviour, such as differentiation, proliferation and alignment [2]. Besides, the membranes for biomimetic models should also feature characteristics such as high porosity for permeability of nutrients to the cells and cell-cell communication. Soft lithography and ion track etching are the most common techniques to fabricate porous membranes for OOCs. These techniques could be combined with fabrication methods that also create microstructures (e.g. soft lithography replica moulding and thermoforming, respectively) to produce more biomimetic membranes. Lastly, phase separation micromoulding and electrospinning are discussed since they could also provide porous membranes with microstructures.

Because of the shortcomings of membranes in organ models, in this thesis, we aimed to develop new membranes based on PTMC which could enable development of more biomimetic lung epithelial-endothelial models. We used the polymer PTMC to fabricate porous membranes since it possesses several relevant properties for our application, such as good mechanical properties (e.g. a significantly lower E-modulus compared to that of PET and PC) [7-13] and good cytocompatibility [8,14,15].

Chapter 3 describes the development of PTMC membranes via TIPS and photo-crosslinking. The membranes were porous and highly permeable, but the pore connectivity and pore diameter varied greatly. The produced pores on the air side were quite large and could stimulate unwanted cell migration through the membrane. Moreover, crosslinking was not very efficient since the membranes had a relatively low gel content. Since the temperature during crosslinking rose above the melting point of the crystallised solvent, which could alter the membrane morphology, we investigated the effect of cooling the membrane during crosslinking. Decreasing the temperature during photo-crosslinking below the melting point of the solvent did not change the surface porosity and average pore diameter. However, it decreased the maximum pore diameter on the air 
side, which is relevant for limiting cell migration. Moreover, the water permeance greatly decreased, probably due to the decrease of the maximum pore diameter on the air side, and the membrane crosslinking improved. Probably, some of the crystallised solvent in the uncooled membranes melted during crosslinking while this was prevented in the cooled membranes, better preserving the structure of the latter. We concluded that TIPS is a relatively simple method to produce porous and permeable PTMC membranes. However, the temperature of the membrane must be kept below the melting point of the solvent during photo-crosslinking. Moreover, to fully utilise these membranes, optimisation of the method is needed to increase their reproducibility.

In Chapter 4, PTMC membranes were fabricated using LIPS. Different properties of the membranes were tailored using PEO and PVP as pore formers during LIPS. Low MW PEO (i.e. $35,000 \mathrm{~g} / \mathrm{mol}$ ) led to non-porous membranes. However, application of 5,000,000 g/mol PEO and 360,000 $\mathrm{g} / \mathrm{mol} \mathrm{PVP}$ resulted in highly porous membranes with large pore diameter and high porosity. PEO and PVP also had a positive effect on the ease of handling of the membranes, although those with PEO were still relatively difficult to handle. In chapter 4 , we also presented a novel method to introduce photo-crosslinking after LIPS, which prevented the collapse of pores when incubated in cell culture medium. Increasing the amount of PVP in the polymer dope improved crosslinking, probably because the crosslinking agents could better penetrate the more porous membranes. From our study, a 3:2 weight ratio of PTMC to PVP was found to provide the best membranes concerning porosity, handling and crosslinking efficiency. These membranes were also permeable to water, although water permeance was relatively low compared to that of commercial PET and PC membranes and showed a large variety. Thus, the combination of LIPS and photo-crosslinking has good potential for developing membranes but, like TIPS, it requires further optimisation to deliver membranes for OOCs. 
Chapter 5 presented a novel way to fabricate PTMC membranes using EIPS. The polymer dope composition was fundamental for achieving porous membranes. In our study, using hexanol as a non-solvent led to the best membrane porosity. PEO was also relevant for enabling pore formation, and it improved membrane handling. We established that the porosity of the membranes increased by raising the ambient humidity and lowering the temperature of the substrate on which the membranes were cast. This was, probably, the result of the evaporative cooling effect [16]. By keeping these factors constant but increasing the amount of hexanol (i.e. from a weight ratio of 1:1 PTMC to hexanol to a ratio of 1:3), we increased the membrane thickness and overall membrane porosity. Simultaneously, their E-modulus (i.e. in the range of $10 \mathrm{MPa}$, thus much lower than that of PET and PC, i.e. both in the range of $2 \mathrm{GPa}$ ) and average pore diameter remained similar. EIPS-based membranes with weight ratios of 1:1 and 1:3 of PTMC to hexanol (i.e. M1 and M3 membranes, respectively) had more homogeneous morphology and were more reproducible than those made by TIPS and LIPS. In general, the pore connectivity of these membranes was high and their water permeance was similar or higher than that of commercial PET and PC membranes used in cell culture inserts. These results, combined with the cytocompatible nature of PTMC and the potential of using cyclic stretch which cannot be done with PET or PC membranes, make the M1 and M3 membranes promising alternatives for PET and PC membranes in cell culturing.

The EIPS-based M1 and M3 membranes developed in chapter 5 were used in Chapter 6, in which we developed a novel airway epithelialendothelial model using Calu-3 cells and LMVECs. A membrane coating of L-DOPA and a subsequent coating of fibronectin, collagen I and albumin (L-DOPA+FN/Col I/BSA) resulted in very good Calu-3 cell layers. Mono-cultures and co-cultures on the M3 membranes were similar to those on commercial PET membranes in terms of ZO-1, CD31 and live/dead staining, as well as electrical resistance. The leakage across the cell layer was also very low. In addition, co-cultures showed improved 
cell viability and barrier function compared to the mono-cultures. The barrier function was also similar between the cultures on M3 and PET membranes. The improved function of the co-cultures was, most probably, caused by paracrine crosstalk between the cells across the membranes. We also showed that EMT and EndoMT were most probably absent in the cultures, as indicated by an absence of vimentin and $\alpha$-SMA expression. Based on these characteristics, we concluded that the application of these PTMC membranes can result in an interesting airway epithelial-endothelial model for lung research and that it could form the basis for more advanced models.

After establishing an airway epithelial-endothelial model on the flat PTMC membranes made by EIPS, in Chapter 7, we fabricated membranes with hemispherical microwells using a combination of EIPS and micromoulding. Using a suitable mould with convex hemispheres made from PVDF, we produced porous membranes with concave microwells that resembled alveoli. Besides, membrane morphology was quite similar to that of flat M1 membranes cast with the same polymer dope composition and co-cultures of Calu-3 cells and LMVECs could be established on the membranes with microwells. Moreover, ZO-1, CD31 and live/dead staining showed similar results to cultures on PET and M1 membranes. This suggests that the co-cultures did well on the PTMC membranes with microwells, which is promising for future experiments with cyclic stretch (which cannot be done with the PET membranes). However, the data also suggest that the microwells had no effect on cell behaviour. Finally, the co-cultures on PTMC membranes with microwells were implemented in a commercial OOC-platform with air- and fluid flow. ZO-1 and CD31 stainings showed results like those in static cell culture inserts, indicating no effect of the air- and fluid flow on expression of these markers. However, Calu-3 cells showed higher cell death in the chip, which suggests that these cells experienced more stress in the chip than in the inserts, probably because of the airflow. 


\section{Outlook:}

\section{TIPS- and LIPS-based membranes}

The TIPS- and LIPS-based membranes in Chapters 3 and 4 showed potential, as they were porous, permeable and form-stable. However, to be able to use them for reliable cell culturing, both membrane types require improvement, most importantly concerning their reproducibility. Better control of the conditions during fabrication can greatly enhance the latter. For the membranes made with TIPS in Chapter 3, a more consistent cooling rate could reduce variation between membranes [17]. Moreover, changing the cooling rate and temperature could tailor the pore diameter and possibly the pore connectivity [17]. Furthermore, the temperature of the entire membrane should be kept below the melting point of the solvent to avoid any morphological changes to the membrane. Besides, the crosslinking efficiency could be increased, e.g. by increasing the PETA and Irgacure to PTMC ratios [8]. Regarding the membranes in Chapter 4, the PVP-containing membranes must detach consistently and at once during LIPS, which could be improved by casting on other materials. A more systematic study with various MWs of PEO and PVP would be useful to tailor membrane properties. Lastly, the composition of the bath with crosslinking agents (i.e. the total volume and concentration and ratio of crosslinking agents) could be optimised to ensure optimal and consistent penetration of crosslinking agents for all membranes.

\section{EIPS-based membranes}

As the EIPS-based membranes in Chapter 5 can already be used for lung epithelial-endothelial models as demonstrated in Chapters 6 and 7, these membranes are more attractive for future research than the TIPS- or LIPSbased membranes. Nonetheless, additional characterisation and adaptation are needed.

In Chapter 5, the stiffness of the flat EIPS-based PTMC membranes was determined, i.e. E-moduli of approximately $10 \mathrm{MPa}$. Although PTMC films with similar E-moduli had desirable mechanical properties such as high elongation at break and low permanent deformation [8], additional 
characterisation of the mechanical properties of the membranes used in Chapters 6 and 7 would be beneficial. This would primarily involve cyclic tests to determine permanent deformation and confirm that the membrane morphology remains intact. These experiments would also show relevant advantages of using PTMC for the membranes instead of PET or PC, since the commercial PET and PC membranes are not able to stretch without deforming permanently.

Although the data suggested a positive effect of LMVECs on performance of the model in Chapter 6, such as the barrier function, CD31 staining did not show a traditional localisation of the CD31. Additional staining of the LMVECs, e.g. for F-actin, could provide better insight into the morphology of the LMVECs. Besides, a Z-stack of the cultures on the microstructured PTMC membranes could confirm if the microwells were indeed covered completely by the Calu-3 cells. Furthermore, in this thesis we used vimentin and $\alpha$-SMA as markers of EMT and EndoMT. Additional stainings for other EMT- and EndoMT-markers, e.g. Ncadherin and fibroblast-specific protein-1 (FSP-1), could support this. Finally, the bronchial Calu-3 cell line was used here to represent lung epithelial cells. In future studies, it would be very interesting to use primary small airway or primary alveolar epithelial cells instead, both on the flat and microstructured PTMC membranes.

\section{Improving cell attachment, pore distribution and mechanical properties}

Although the PTMC membranes used in Chapters 6 and 7 were porous and permeable, and co-cultures could be established on them, certain aspects of both membrane types could be improved, i.e. cell attachment, and the porosity and mechanical properties of the membranes. This could be achieved by changing several of the components of the polymer dope. These improvements would be particularly interesting for future research with primary lung epithelial cells, since these are generally less robust than the Calu-3 cells. 
Improvement of cell attachment

While cell attachment of Calu-3 cells and LMVECs was good on LDOPA+FN/Col I/BSA-coated PTMC membranes, Calu-3 cell attachment was low on uncoated and FN/Col I/BSA-coated PTMC membranes. Decreasing the amount of PEO would probably improve cell adhesion. Since PEO is hydrophilic, it probably caused low adsorption of proteins from culture medium or protein solutions directly onto the membranes, resulting in the low attachment of Calu-3 cells on (uncoated) PTMC membranes in Chapter 5. Alternatively, the MW of the PEO could be decreased, which could increase the distribution of the PEO across the membrane, thus preventing PEO-rich regions on the membrane surface, thereby stimulating cell adhesion.

\section{More homogeneous pore distribution}

Establishing a more homogeneous pore distribution could stimulate cell growth and performance (e.g. barrier formation) due to more homogeneous nourishment. This is particularly interesting for the microstructured membranes of Chapter 7 , which were denser at the base of the microstructures. This could be achieved by lowering the MW or concentration of PEO.

Alternatively, as discussed in Chapter 2, ion track etching and soft lithography can create pores with a homogeneous pore size $[2,18]$. Moreover, ion track etching can provide a relatively homogeneous porosity, while soft lithography gives complete control over the position of pores [2,18]. In addition, there is no need for (non-cytocompatible) additives. However, to create membranes with microwells and homogeneously distributed pores, these techniques would have to be performed in a separate step before micromoulding by e.g. thermoforming $[2,18]$, followed by crosslinking.

In contrast, particulate leaching can be easily combined with micromoulding [2]. A polymer solution containing micrometre-sized particulates can be cast over a mould, after which the membranes are crosslinked and the particulates are washed away to result in porous and 
microstructured membranes. Particulate leaching also does not require additives that may hamper cell attachment. However, the particulates have to be small enough to prevent cell migration, i.e. up to several micrometres, such as the calcium carbonate particulates of approximately $0.56 \mu \mathrm{m}$ used by Guo et al. [19].

Co-polymers and natural materials for more tissue-like mechanical properties and cytocompatibility

The EIPS-based membranes in Chapter 5 had E-moduli in the range of 10 $\mathrm{MPa}$, still relatively high compared to the stiffness of lungs (i.e. $400 \mathrm{~Pa}$ or lower) [4-6]. Stiffness of the membranes should be lowered to establish more biomimetic membranes. A lower membrane stiffness could be achieved by replacing the PTMC with co-polymers or other materials.

Instead of adding PEO as an additive, co-polymers of PTMC and PEO could be used. Networks of these co-polymers had E-moduli ranging from $18 \mathrm{kPa}$ to $2.5 \mathrm{MPa}$ [9]. Using similar polymer networks, we could fabricate membranes with a stiffness that is much closer to that of lung tissue. It would also ensure homogeneous distribution of PEO throughout the membranes with similar benefits on e.g. porosity, as described above.

Recently, there has been much attention for the use of natural materials, mostly ECM proteins such as collagen and Matrigel, in the field of LOCs and lung models [20,21]. Unsurprisingly, membranes and hydrogels made with these materials have many desirable characteristics such as high cytocompatibility and mechanical properties that (more) closely resemble the targeted tissue [20]. Moreover, in many of these studies, the proteins are organised in fibrous meshes which resemble the structure of the ECM [20-22]. Zamprogno et al. fused fibrous and porous membranes made from collagen and elastin on top of thin gold honeycomb meshes with hexagonal openings of $225 \mu \mathrm{m}$, in the range of the size of alveoli [22]. Because of this, a negative pressure underneath the membranes in an LOC resulted in deformation of the membrane, only in the hexagonal openings, resembling expansion of alveoli during respiration. However, even with these natural materials, the exact composition of the membrane is still 
important [20,21]. This could be complicated by the batch-to-batch variation of natural materials such as Matrigel.

Using polymers such as PTMC gives more control over the membrane composition and usually results in much tougher constructs than those made from natural proteins [23]. Liang et al. electrospun fibrous mats, consisting of crosslinked PTMC and gelatin, with desirable properties such as low E-moduli (i.e. less than $1 \mathrm{MPa}$ ), high toughness and cytocompatibility with hMSCs [23]. This is very interesting for OOC membranes subjected to cyclic stretch and for mimicking the mechanical properties of lung tissue. Moreover, the fibrous mats had a high porosity and their fibrous nature resembled the structure of the ECM. Furthermore, as we discussed in Chapter 2, electrospinning can be used together with moulds to include microstructures. This could result in tough, highly porous membranes with microstructures and a surface morphology which resembles the alveoli or other tissue in the lung.

\section{Adaptations of the microstructures}

In Chapter 7, we did not see an effect of the microwells of the microstructured membranes on cell behaviour. This was probably because the microwells were relatively shallow, despite the overall good replication of the microwells from the mould. Creating a mould with higher convex microstructures should increase the final depth of the microwells in the PTMC membranes. Moreover, a systematic study is needed to investigate the effects of microwells with different degrees of curvature on cell behaviour, since the amount of curvature can influence the magnitude of the cell response [24]. Ideally, these tests would be performed both in static conditions and dynamic conditions (i.e. with airflow, fluid flow and/or cyclic stretch of the membranes and cells). Since we did not see a clear effect of the air- and fluid flow, the flow rates should also be varied. This way, possible synergistic effects of the surface curvature, cyclic stretch, and air- and fluid flow could be investigated. 


\section{Cyclic stretch of microstructured membranes in an OOC}

During respiration, the alveoli expand due to expansion of the thoracic cavity, which creates a negative pressure which is mimicked by the LOC from Zamprogno et al., containing a collagen-elastin membrane on a gold honeycomb mesh [22]. They determined that their model results in a $10 \%$ linear strain of the membrane, similar to that of the alveoli, when expanded. Introducing a similar negative pressure to the chip and microstructured membrane presented in Chapter 7 will move and deform the membrane, but a homogeneous $10 \%$ strain across the surface of the membrane is unlikely. Therefore, a support such as the mesh used by Zamprogno et al. could be valuable to prevent deformation of the membrane in between the microwells, and stimulate expansion of the microwells [22].

\section{General conclusion and outlook:}

In this thesis, we show the development of PTMC membranes, based on phase separation (especially EIPS) and micromoulding, for novel biomimetic lung epithelial-endothelial models. We demonstrated that they are suitable for an airway epithelial-endothelial model in a microfluidic chip. Moreover, we showed and discussed the adaptability of the fabrication methods to tailor membrane properties, thus enabling future adaptation of the membranes to novel insights for implementation in advanced biomimetic models.

\section{References}

1. World Health Organization. The Top 10 Causes of Death. Available online: https://www.who.int/news-room/fact-sheets/detail/the-top-10-causes-of-death (accessed on 12 August 2021).

2. Chapter 2 of this thesis.

3. Huh, D.; Matthews, B.D.; Mammoto, A.; Montoya-Zavala, M.; Hsin, H.Y.; Ingber, D.E. Reconstituting organ-level lung functions on a chip. Science. 2010, 328(5986), 1662-1668. DOI: 10.1126/science.1188302.

4. Lee, J.H.; Lee, S.J.; Khang, G.; Lee, H.B. Interaction of fibroblasts on polycarbonate membrane surfaces with different micropore sizes and 
hydrophilicity. J Biomater. Sci. Polym. Ed. 1999, 10(3), 283-294. DOI: 10.1163/156856299X00351.

5. Gotoh, K.; Yasukawa, A.; Taniguchi, K. Water contact angles on poly(ethylene terephthalate) film exposed to atmospheric pressure plasma. J Adhes. Sci. Technol. 2011, 25(1-3), 307-322. DOI: 10.1163/016942410X511114.

6. Butcher, D.T.; Alliston, T.; Weaver, V.M. A tense situation: forcing tumour progression. Nat. Rev. Cancer. 2009, 9(2), 108-122. DOI: 10.1038/nrc2544.

7. Bat, E.; Feijen, J.; Grijpma, D.W. Biodegradable elastomeric networks: highly efficient cross-linking of poly(trimethylene carbonate) by gamma irradiation in the presence of pentaerythritol triacrylate. Biomacromolecules. 2010, 11(10), 2692-2699. DOI: 10.1021/bm1007234.

8. Bat, E.; Kothman, B.H.M.; Higuera, G.A.; van Blitterswijk, C.A.; Feijen, J.; Grijpma, D.W. Ultraviolet light crosslinking of poly(trimethylene carbonate) for elastomeric tissue engineering scaffolds. Biomaterials. 2010, 31(33), 8696-8705. DOI: 10.1016/j.biomaterials.2010.07.102.

9. Allijn, I.; Ribeiro, M.; Poot, A.A.; Passier, R.; Stamatialis, D. Membranes for Modelling Cardiac Tissue Stiffness In Vitro Based on Poly(trimethylene carbonate) and Poly(ethylene glycol) Polymers. Membranes. 2020, 10(10), E274. DOI: $10.3390 /$ membranes10100274.

10. Schüller-Ravoo, S.; Feijen, J.; Grijpma, D.W. Flexible, elastic and tear-resistant networks prepared by photo-crosslinking poly(trimethylene carbonate) macromers. Acta. Biomater. 2012, 8(10), 3576-3585. DOI: 10.1016/j.actbio.2012.06.004.

11. Pêgo, A.P.; Poot, A.A.; Grijpma, D.W.; Feijen, J. Copolymers of trimethylene carbonate and epsilon-caprolactone for porous nerve guides: synthesis and properties. Biomater. Sci. Polym. Ed. 2001, 12(1), 35-53. DOI: 10.1163/156856201744434.

12. Zant, E.; Bosman, M.J.; Grijpma, D.W. Combinatorial synthesis of photocrosslinked biodegradable networks. J. Appl. Biomater. Funct. Mater. 2012, 10(3), 197-202. DOI: 10.5301/JABFM.2012.10344.

13. Bat, E.; van Kooten, T.G.; Feijen, J.; Grijpma, D.W. Resorbable elastomeric networks prepared by photocrosslinking of high-molecular-weight poly(trimethylene carbonate) with photoinitiators and poly(trimethylene carbonate) macromers as crosslinking aids. Acta. Biomater. 2011, 7(5), 19391948. DOI: 10.1016/j.actbio.2011.01.010.

14. Pêgo, A.P.; Siebum, B.; Van Luyn, M.J.; Gallego y Van Seijen, X.J.; Poot, A.A.; Grijpma, D.W.; Feijen, J. Preparation of degradable porous structures based on 1,3-trimethylene carbonate and D,L-lactide (co)polymers for heart tissue engineering. Tissue Eng. 2003, 9(5), 981-994. DOI: 10.1089/107632703322495628.

15. Papenburg, B.J.; Schuller-Ravoo, S.; Bolhuis-Versteeg, L.A.M.; Hartsuiker, L.; Grijpma, D.W.; Feijen, J.; Wessling, M.; Stamatialis, D. Designing porosity and topography of poly(1,3-trimethylene carbonate) scaffolds. Acta. Biomater. 2009, 5(9), 3281-3294. DOI: 10.1016/j.actbio.2009.05.017. 
16. Pervin, R.; Ghosh, P.; Basavaraja, M.G. Tailoring pore distribution in polymer films via evaporation induced phase separation. RSC Adv. 2019, 9, 15593-15605. DOI: 10.1039/C9RA01331H.

17. Mannella, G.A.; Conoscenti, G.; Carfi Pavia, F.; La Carrubba, V.; Brucato, V. Preparation of polymeric foams with a pore size gradient via Thermally Induced Phase Separation (TIPS). Materials Letters. 2015, 160, 31-33. DOI: 10.1016/j.matlet.2015.07.055.

18. Baptista, D.; Teixeira, L.M.; Birgani, Z.T.; van Riet, S.; Pasman, T.; Poot, A.; Stamatialis, D.; Rottier, R.J.; Hiemstra, P.S.; Habibović, P.; van Blitterswijk, C.; Giselbrecht, S.; Truckenmüller, R. 3D alveolar in vitro model based on epithelialized biomimetically curved culture membranes. Biomaterials. 2021, 266, 120436. DOI: 10.1016/j.biomaterials.2020.120436.

19. Guo, Z.; Grijpma, D.; Poot, A. Leachable Poly(Trimethylene Carbonate) $/ \mathrm{CaCO}_{3}$ Composites for Additive Manufacturing of Microporous Vascular Structures. Materials (Basel, Switzerland). 2020, 13(15), 3435. DOI: 10.3390/ma13153435.

20. Bennet, T.J.; Randhawa, A.; Hua, J.; Cheung, K.C. Airway-On-A-Chip: Designs and Applications for Lung Repair and Disease. Cells. 2021, 10(7), 1602. DOI: 10.3390/cells10071602.

21. Mondrinos, M.J.; Yi, Y.S.; Wu, N.K.; Ding, X.; Huh, D. Native extracellular matrix-derived semipermeable, optically transparent, and inexpensive membrane inserts for microfluidic cell culture. Lab on a chip. 2017, 17(18), 31463158. DOI: 10.1039/c7lc00317j.

22. Zamprogno, P.; Wüthrich, S.; Achenbach, S.; Thoma, G.; Stucki, J.D.; Hobi, N.; Schneider-Daum, N.; Lehr, C.M.; Huwer, H.; Geiser, T.; Schmid, R.A.; Guenat, O.T. Second-generation lung-on-a-chip with an array of stretchable alveoli made with a biological membrane. Communications biology. 2021, 4(1), 168. DOI: 10.1038/s42003-021-01695-0.

23. Liang, J.; Chen, H.; Guo, Z.; Dijkstra, P.; Grijpma, D.; Poot, A. Tough fibrous mats prepared by electrospinning mixtures of methacrylated poly(trimethylene carbonate) and methacrylated gelatin. European Polymer Journal. 2021, 152, 110471. DOI: 10.1016/j.eurpolymj.2021.110471.

24. Soscia, D.A.; Sequeira, S.J.; Schramm, R.A.; Jayarathanam, K.; Cantara, S.I.; Larsen, M.; Castracane, J. Salivary gland cell differentiation and organization on micropatterned PLGA nanofiber craters. Biomaterials. 2012, 34(28), 6773-6784. DOI: 10.1016/j.biomaterials.2013.05.061. 
- Chapter 8 -

Conclusions and future outlook 


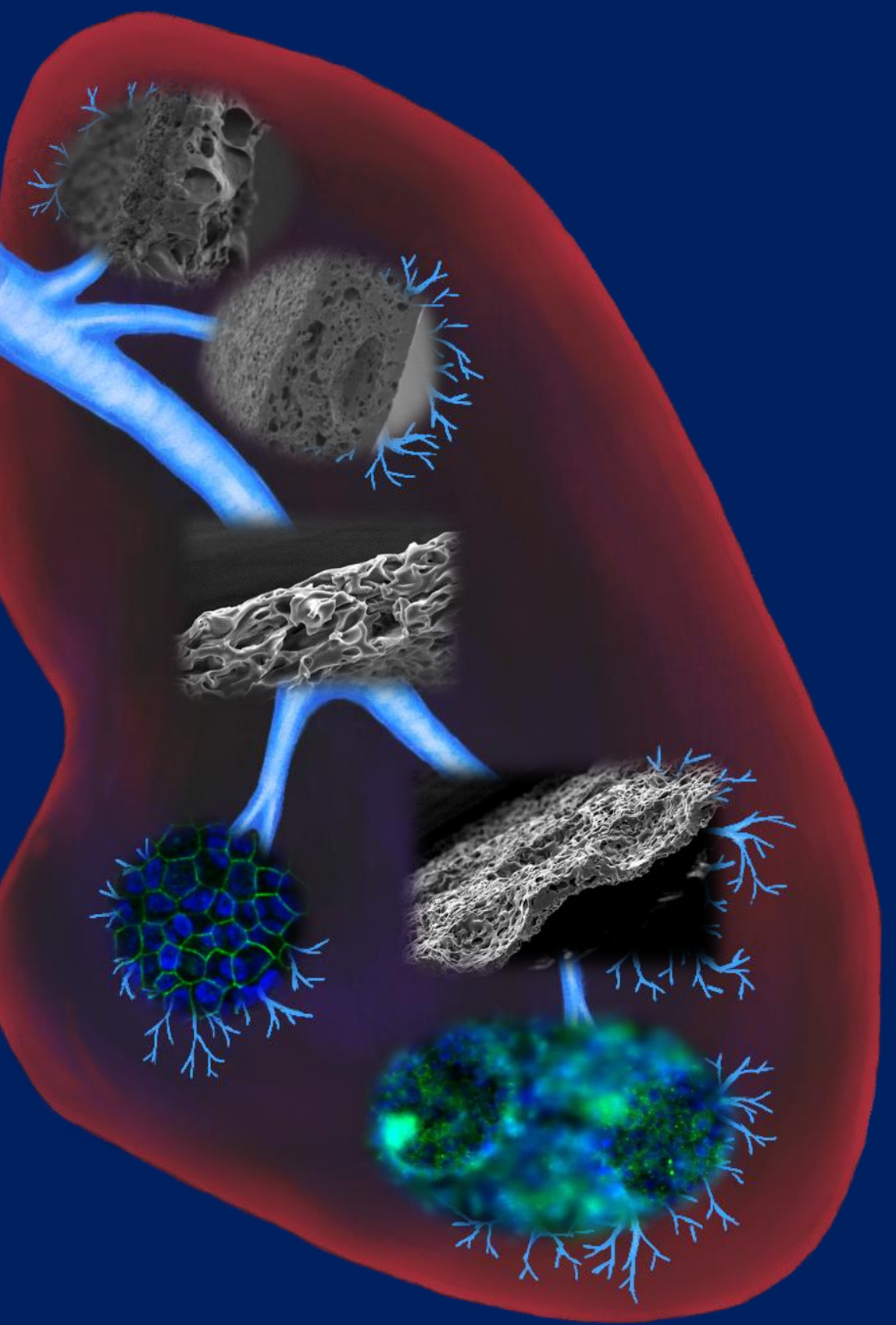




\section{- Summary -}

Thijs Pasman, Dimitrios Stamatialis and André A. Poot

Technical Medical (TechMed) Centre, Department of Biomaterials Science and Technology, Faculty of Science and Technology, University of Twente, 7522 NB Enschede, The Netherlands 
The lungs govern the exchange of oxygen and carbon dioxide in their alveoli which is essential for the functioning of our cells. Thus, damage to the lungs can be life-threatening, which is reflected in the fact that lung diseases are one of the most common causes of death. This has become especially apparent with the recent pandemic involving the SARS-CoV-2 virus. For the treatment of lung diseases, lung models that generate reliable data of potential therapies are very much needed. Unfortunately, traditional in vitro models based on cell cultures and animal models do not properly reflect the human lung and often provide limited reliability. As a result, lungs-on-chips (LOCs) were developed. LOCs are small cell culture models that aim to mimic functions of the human lungs including dynamic phenomena such as air- and fluid flow. Moreover, they can be used with human cells and avoid ethical issues, which are significant disadvantages of animal models.

Generally, cells in traditional in vitro lung models and LOCs are grown on porous membranes. However, often there is little attention for the membrane properties, such as good cytocompatibility, mechanical properties matching those of the tissue, and high porosity and pore connectivity, which are important for providing a biomimetic lung model. The shortcomings of the currently used membranes in lung models motivated the research of this thesis, in which we addressed those limitations and described the development of novel membranes for biomimetic lung models and LOCs.

Chapter 1 provided a detailed introduction to the topic of this thesis and presented the aim, scope and outline of the thesis and chapters.

In Chapter 2, we discussed that material properties, such as hydrophilicity and surface topography, affect cell adhesion, proliferation and differentiation. We reviewed membranes used in LOCs and organson-chips (OOCs) in general, most often made from poly(ethylene terephthalate) (PET) or poly(carbonate) (PC) by (ion) track etching, or poly(dimethyl siloxane) (PDMS) by soft lithography. These materials, techniques and membranes have relevant advantages, such as high 
control over pore size. However, they also have disadvantages, such as the high stiffness of PC and PET compared to that of most tissues. Moreover, the membranes in OOCs are mostly flat, lacking microstructures that resemble a tissue's structure, e.g. the spherical nature of alveoli. Therefore, techniques such as thermoforming, soft lithography replica moulding and phase separation micromoulding were presented for creating porous and microstructured membranes that better reflect the nature of the tissue.

In Chapters 3 - 5, we described the development and characterisation of porous membranes based on linear high molecular weight poly(trimethylene carbonate) (PTMC) for lung epithelial-endothelial models using different phase separation techniques. PTMC has good cytocompatibility and has mechanical properties that are more reminiscent of most tissues than those of PC and PET. Moreover, PTMC has been used extensively to fabricate films, membranes and scaffolds for biomedical applications.

In Chapter 3, porous PTMC membranes were made using temperatureinduced phase separation (TIPS), followed by photo-crosslinking. The membranes contained micrometre-sized pores and were highly permeable to water. However, the membrane morphology was quite heterogeneous, and the crosslinking efficiency was rather low. Cooling the membranes during crosslinking resulted in more homogeneous membranes and better crosslinking efficiency. Besides, although still high, the water permeance decreased drastically when the membranes were cooled during crosslinking. We show that there is potential for TIPS for the preparation of porous PTMC membranes, although further optimisation would be required.

Chapter 4 presented the fabrication of porous PTMC membranes by means of liquid-induced phase separation (LIPS) with poly(ethylene oxide) (PEO) or poly(vinyl pyrrolidone) (PVP) as pore formers. Increasing the molecular weight and/or concentration of the pore formers increased 
pore size and porosity. By introducing crosslinking agents after LIPS, the membranes could be photo-crosslinked, which is a novel approach. This way, porous and form-stable PTMC membranes that are highly permeable to water could be made. However, optimisation of the fabrication to reduce variation between membranes is desired before they are applied in cell culture models.

In Chapter 5, we developed porous PTMC membranes using evaporation-induced phase separation (EIPS) with alcohols as nonsolvents, followed by photo-crosslinking. The composition of the polymer dope and the environmental conditions during membrane casting and EIPS were essential for the production of these membranes. Using hexanol as a non-solvent resulted in porous membranes, while the use of smaller, more volatile alcohols yielded less- or non-porous membranes. Pore formation was stimulated further by including PEO, increasing the ambient humidity and cooling the membranes during EIPS. Besides, using $600 \mathrm{~kg} / \mathrm{mol}$ PTMC led to homogeneous membranes, while 1,300 $\mathrm{kg} / \mathrm{mol}$ PTMC produced more heterogeneous membranes. Using the 600 $\mathrm{kg} / \mathrm{mol}$ PTMC, we could fabricate PTMC membranes with relevant pore size and porosity with good reproducibility. Moreover, the membranes were form-stable, highly permeable to water and had E-moduli that better represent that of the lungs than those of PET and PC. Furthermore, by varying the hexanol amount in the polymer dope, we could tailor the membrane pore connectivity. These results suggest a potential for the use of these membranes in organ models.

Therefore, in Chapter 6 we applied two types of membranes developed in Chapter 5 in a lung epithelial-endothelial model with Calu-3 lung epithelial cells and human lung microvascular endothelial cells (LMVECs). Initial Calu-3 cell attachment studies showed that coating of the membranes with L-DOPA and subsequently fibronectin, collagen I and bovine serum albumin was necessary, likely because the PEO in the membranes limited cell attachment. Calu-3 cells showed good expression of Zonula Occludens 1 (ZO-1), a tight junction protein, while LMVECs 
were CD31-positive, albeit the CD31 was not localised at the cell membrane. The cell cultures had good viability. Electrical resistance measurements and fluorescein isothiocyanate-dextran (FITC-dextran) permeability assays showed good barrier functions of Calu-3 cell monocultures and co-cultures on the PTMC membranes. Moreover, barrier function and cell viability of co-cultures were better than those of monocultures. Based on stainings of vimentin and $\alpha$-smooth muscle actin $(\alpha-$ SMA), we did not find signs of epithelial- and endothelial-tomesenchymal transitions (EMT and EndoMT, respectively). These results show potential for these membranes to be used in organ models. We concluded that the model can act as a basis for biomimetic airway epithelial-endothelial and alveolar-endothelial models.

In Chapter 7, we developed microstructured PTMC membranes by combining micromoulding and EIPS. As a result, we could successfully introduce microwells, mimicking the lung alveoli, to the membranes. Moreover, these microstructured membranes were integrated in a commercial OOC platform that allowed air- and fluid flow. Calu-3 cells and LMVECs were successfully cultured on the membranes and the cells were ZO-1- and CD31-positive, respectively. Cells grown on the microstructured membranes had good viability in both cell culture inserts and chips. The co-cultures in the inserts also formed a good barrier since they had a high electrical resistance and low permeability of FITCdextran. Future investigation of the model in a LOC would be desirable, focusing on the effects of combining the microstructures, air- and fluid flow, and preferably also cyclic stretch of the membranes, on cell behaviour. The results presented in this chapter show that EIPS combined with micromoulding has great potential for the development of suitable membranes for biomimetic lung models and organ models in general.

Chapter 8 presented an overview of the main conclusions of this thesis and provided an outlook for further analysis, application and improvements of the presented membranes and cell culture models. 
- Samenvatting -

- Samenvatting - 
De longen regelen de uitwisseling van zuurstof en koolstofdioxide in de longblaasjes, wat essentieel is voor het functioneren van onze cellen. Zo kan schade aan de longen levensbedreigend zijn, wat tot uiting komt in het feit dat longziekten één van de meest voorkomende doodsoorzaken zijn. Dit is vooral duidelijk geworden met de recente pandemie waarbij het SARS-CoV-2-virus betrokken is. Longmodellen die betrouwbare gegevens van mogelijke therapieën genereren zijn hard nodig voor de behandeling van longziekten. Helaas weerspiegelen traditionele in vitromodellen op basis van celculturen en diermodellen de menselijke long niet goed en bieden ze vaak een beperkte betrouwbaarheid. Als gevolg hiervan werden longen-op-chips (LOCs) ontwikkeld. LOCs zijn kleine celcultuurmodellen die tot doel hebben functies van de menselijke longen na te bootsen, inclusief dynamische verschijnselen zoals lucht- en vloeistofstroom. Bovendien kunnen ze worden gebruikt met menselijke cellen en voorkomen ze ethische problemen, wat belangrijke nadelen zijn van diermodellen.

Over het algemeen worden cellen in traditionele in vitro longmodellen en LOCs gekweekt op poreuze membranen. Echter, vaak is er weinig aandacht voor de membraaneigenschappen, zoals goede cytocompatibiliteit, mechanische eigenschappen die overeenkomen met die van het weefsel, en hoge porositeit en verbinding tussen poriën, die belangrijk zijn voor het ontwikkelen van een biomimetisch longmodel. De tekortkomingen van de momenteel gebruikte membranen in longmodellen motiveerden het onderzoek van dit proefschrift, waarin we deze beperkingen aan de orde stelden en de ontwikkeling van nieuwe membranen voor biomimetische longmodellen en LOCs beschreven.

Hoofdstuk 1 gaf een gedetailleerde inleiding op het onderwerp van dit proefschrift en presenteerde het doel, het kader en de opzet van het proefschrift en de hoofdstukken.

In Hoofdstuk 2 bespraken we dat materiaaleigenschappen, zoals hydrofiliciteit en oppervlaktetopografie, de adhesie, proliferatie en differentiatie van cellen beïnvloeden. We hebben membranen besproken 
die worden gebruikt in LOCs en organen-op-chips (OOCs) in het algemeen, meestal gemaakt van poly(ethyleen tereftalaat) (PET) of poly(carbonaat) (PC) door (ion) track etching, of poly(dimethyl siloxaan) (PDMS) door zachte lithografie. Deze materialen, technieken en membranen hebben relevante voordelen, zoals een hoge controle over de poriegrootte. Ze hebben echter ook nadelen, zoals de hoge stijfheid van PC en PET in vergelijking met die van de meeste weefsels. Bovendien zijn de membranen in OOCs meestal vlak, zonder microstructuren die lijken op de structuur van een weefsel, bijvoorbeeld de sferische vorm van longblaasjes. Daarom werden technieken zoals thermovormen, replicagieten met zachte lithografie en micro-moulding met fasescheiding gepresenteerd voor het creëren van poreuze membranen met microstructuren die de structuur van het weefsel beter nabootsen.

In Hoofdstukken 3-5 beschreven we de ontwikkeling en karakterisering van poreuze membranen op basis van lineair hoogmoleculair gewicht poly(trimethyleen carbonaat) (PTMC) voor longepitheel-endotheel modellen met behulp van verschillende fasescheidingstechnieken. PTMC heeft een goede cytocompatibiliteit en heeft mechanische eigenschappen die meer lijken op dat van de meeste weefsels dan die van PC en PET. Bovendien is PTMC op grote schaal gebruikt om films, membranen en scaffolds voor biomedische toepassingen te fabriceren.

In Hoofdstuk 3 werden poreuze PTMC-membranen gemaakt met behulp van temperatuur-geïnduceerde fasescheiding (TIPS), gevolgd door fotocrosslinking. De membranen bevatten poriën ter grootte van micrometers en waren zeer goed doorlatend voor water. De membraanmorfologie was echter behoorlijk heterogeen en de efficiëntie van het crosslinken was vrij laag. Het afkoelen van de membranen tijdens crosslinken resulteerde in meer homogene membranen en een hogere efficiëntie van het crosslinken. Bovendien, hoewel nog steeds hoog, nam de doorlatendheid voor water drastisch af wanneer de membranen werden gekoeld tijdens crosslinking. We laten zien dat er potentieel is voor TIPS voor de bereiding van poreuze PTMC-membranen, hoewel verdere optimalisatie vereist zou zijn. 
Hoofdstuk 4 presenteerde de fabricage van poreuze PTMC-membranen door middel van vloeistof-geïnduceerde fasescheiding (LIPS) met poly(ethyleen oxide) (PEO) of poly(vinyl pyrrolidon) (PVP) als porievormers. Verhoging van het molecuulgewicht en/of de concentratie van de porievormers vergrootte de poriegrootte en porositeit. Door na LIPS stoffen te introduceren die crosslinken mogelijk maken, kunnen de membranen worden gecrosslinkt. Dit is een nieuwe benadering. Op deze manier konden poreuze en vormstabiele PTMC-membranen worden gemaakt die zeer goed doorlatend zijn voor water. Optimalisatie van de fabricage om variatie tussen membranen te verminderen is echter gewenst voordat ze worden toegepast in celkweekmodellen.

In Hoofdstuk 5 hebben we poreuze PTMC-membranen ontwikkeld met behulp van verdamping-geïnduceerde fasescheiding (EIPS) met alcoholen als niet-oplosmiddelen, gevolgd door foto-crosslinking. De samenstelling van de polymeeroplossing en de omstandigheden van de omgeving tijdens het membraangieten en EIPS waren essentieel voor de productie van deze membranen. Het gebruik van hexanol als nietoplosmiddel resulteerde in poreuze membranen, terwijl het gebruik van kleinere, meer vluchtige alcoholen minder of niet-poreuze membranen opleverde. Porievorming werd verder gestimuleerd door PEO toe te voegen, de luchtvochtigheid te verhogen en de membranen te koelen tijdens EIPS. Bovendien leidde het gebruik van $600 \mathrm{~kg} / \mathrm{mol}$ PTMC tot homogene membranen, terwijl $1.300 \mathrm{~kg} / \mathrm{mol}$ PTMC meer heterogene membranen produceerden. Met behulp van de $600 \mathrm{~kg} / \mathrm{mol}$ PTMC konden we PTMC-membranen fabriceren met relevante poriegrootte en porositeit met een goede reproduceerbaarheid. Bovendien waren de membranen vormstabiel, goed doorlatend voor water en hadden ze E-moduli die beter overeenkwamen met die van de longen dan die van PET en PC. Bovendien konden we, door de hoeveelheid hexanol in de polymeeroplossing te variëren, de verbinding tussen de poriën van de membranen aanpassen. Deze resultaten suggereren een potentieel voor het gebruik van deze membranen in orgaanmodellen. 
Daarom hebben we in Hoofdstuk 6 twee typen membranen, ontwikkeld in Hoofdstuk 5, toegepast in een longepitheel-endotheelmodel met Calu3 longepitheelcellen en humane long microvasculaire endotheelcellen (LMVECs). Initiële Calu-3 celhechtingsstudies toonden aan dat het coaten van de membranen met L-DOPA en vervolgens fibronectine, collageen I en albumine uit het serum van runderen noodzakelijk was, waarschijnlijk omdat de PEO in de membranen de celhechting beperkte. Calu-3-cellen vertoonden een goede expressie van Zonula Occludens 1 (ZO-1), een tight junction-eiwit, terwijl LMVECs CD31-positief waren, hoewel de CD31 niet gelokaliseerd was rond het celmembraan. De celkweken hadden een goede levensvatbaarheid. Elektrische weerstandsmetingen en testen voor de doorlatendheid voor fluoresceïne-isothiocyanaat-dextran (FITCdextran) lieten goede barrièrefuncties zien van mono-culturen van Calu3 cellen en co-culturen op de PTMC-membranen. Bovendien waren de barrièrefunctie en levensvatbaarheid van cellen van de co-culturen beter dan die van monoculturen. Op basis van kleuringen van vimentine en $\alpha$ gladde spieractine ( $\alpha$-Smooth Muscle Actin of $\alpha$-SMA), vonden we geen tekenen van epitheel- en endotheel-naar-mesenchymale overgang (respectievelijk EMT en EndoMT). Deze resultaten laten zien dat deze membranen kunnen worden gebruikt in orgaanmodellen. We concludeerden dat het model kan dienen als basis voor biomimetische luchtwegepitheel-endotheel en alveolaire-endotheel modellen.

In Hoofdstuk 7 hebben we PTMC-membranen met microstructuren ontwikkeld door micromoulding en EIPS te combineren. Als gevolg hiervan konden we met succes microwellen, die de longblaasjes nabootsen, introduceren in de membranen. Bovendien werden deze membranen met microstructuren geïntegreerd in een commercieel OOCplatform dat lucht- en vloeistofstroom mogelijk maakte. Calu-3-cellen en LMVECs werden met succes gekweekt op de membranen en de cellen waren respectievelijk ZO-1- en CD31-positief. Cellen gekweekt op de membranen met microstructuren hadden een goede levensvatbaarheid in zowel celkweekinserts als chips. De co-culturen in de inserts vormden 
ook een goede barrière aangezien ze een hoge elektrische weerstand en lage doorlatendheid voor FITC-dextran hadden. Toekomstig onderzoek van het model in een LOC zou wenselijk zijn, gericht op de effecten van het combineren van de microstructuren, lucht- en vloeistofstroom, en bij voorkeur ook cyclische rek van de membranen, op het celgedrag. De resultaten die in dit hoofdstuk worden gepresenteerd laten zien dat EIPS in combinatie met micromoulding een groot potentieel heeft voor de ontwikkeling van geschikte membranen voor biomimetische longmodellen en orgaanmodellen in het algemeen.

Hoofdstuk 8 geeft een overzicht van de belangrijkste conclusies van dit proefschrift en biedt een vooruitzicht voor verdere analyse, toepassing en verbeteringen van de gepresenteerde membranen en celcultuurmodellen. 


\section{- List of abbreviations -}

\begin{tabular}{|c|c|}
\hline$\alpha-\mathrm{SMA}$ & $\alpha$-smooth muscle actin \\
\hline$\varepsilon-\mathrm{CL}$ & $\varepsilon$-caprolactone \\
\hline$\mu \mathrm{W}$ & microwell \\
\hline${ }^{1} \mathrm{H}-\mathrm{NMR}$ & Proton Nuclear Magnetic Resonance Spectroscopy \\
\hline ALI & air-liquid interface \\
\hline ARDS & acute respiratory distress syndrome \\
\hline ASCs & adipose stem cells \\
\hline BBB & blood-brain-barrier \\
\hline BPD & bronchopulmonary dysplasia \\
\hline BSA & bovine serum albumin \\
\hline Col I & collagen I \\
\hline COPD & chronic obstructive pulmonary disease \\
\hline DMEM & Dulbecco's Modified Eagle's Medium \\
\hline DPBS & Dulbecco's PBS without $\mathrm{Ca}^{2+} / \mathrm{Mg}^{2+}$ \\
\hline $\mathrm{EC}$ & ethylene carbonate \\
\hline ECM & extracellular matrix \\
\hline EIPS & evaporation-induced phase separation \\
\hline EMEM & Eagle's Minimum Essential Medium \\
\hline E-moduli & elastic moduli \\
\hline EMT & epithelial-to-mesenchymal transition \\
\hline EndoMT & endothelial-to-mesenchymal transition \\
\hline FA & formaldehyde \\
\hline FBS & fetal bovine serum \\
\hline FITC-dextran & fluorescein isothiocyanate-dextran \\
\hline $\mathrm{FN}$ & fibronectin \\
\hline $\mathrm{FN} / \mathrm{Col} \mathrm{I} / \mathrm{BSA}$ & fibronectin, collagen $\mathrm{I}$, and bovine serum albumin \\
\hline FSP-1 & fibroblast-specific protein-1 \\
\hline GPC & gel permeation chromatography \\
\hline HGFs & human gingival fibroblasts \\
\hline hiPSCs & human-induced pluripotent stem cells \\
\hline HUVECS & human umbilical vein endothelial cells \\
\hline L-DOPA & levodopa \\
\hline LIPS & liquid-induced phase separation \\
\hline LMVECs & human lung microvascular endothelial cells \\
\hline
\end{tabular}




\begin{tabular}{|c|c|}
\hline LOCs & lungs-on-chips \\
\hline M0 & $\begin{array}{l}\text { PTMC membranes fabricated with EIPS without using a } \\
\text { non-solvent (i.e. hexanol) }\end{array}$ \\
\hline M1 & $\begin{array}{l}\text { PTMC membranes fabricated with EIPS using hexanol as a } \\
\text { non-solvent in a 1:1 (w/w) PTMC to hexanol ratio }\end{array}$ \\
\hline M2 & $\begin{array}{l}\text { PTMC membranes fabricated with EIPS using hexanol as a } \\
\text { non-solvent in a 1:2 (w/w) PTMC to hexanol ratio }\end{array}$ \\
\hline M3 & $\begin{array}{l}\text { PTMC membranes fabricated with EIPS using hexanol as a } \\
\text { non-solvent in a 1:3 (w/w) PTMC to hexanol ratio }\end{array}$ \\
\hline $\mathrm{M}_{30^{\circ} \mathrm{C}}$ & $\begin{array}{l}\text { PTMC membranes fabricated with TIPS and crosslinked at a } \\
\text { maximum temperature of } 30^{\circ} \mathrm{C}\end{array}$ \\
\hline $\mathrm{M} 39^{\circ} \mathrm{C}$ & $\begin{array}{l}\text { PTMC membranes fabricated with TIPS and crosslinked at a } \\
\text { maximum temperature of } 39^{\circ} \mathrm{C}\end{array}$ \\
\hline Mn & number average molecular weight \\
\hline MOC & multi-organ-on-chip \\
\hline MSCs & mesenchymal stem cells \\
\hline Mv & weight average molecular weight \\
\hline MW & molecular weight \\
\hline OOCs & organs-on-chips \\
\hline $\mathrm{P} / \mathrm{S}$ & penicillin-streptomycin \\
\hline PAA & poly(acrylamide) \\
\hline Papp & apparent permeability \\
\hline PC & poly(carbonate) / poly(bisphenol-A-carbonate) \\
\hline PC0.4 & PC membranes with $0.4 \mu \mathrm{m}$ pores \\
\hline PCL & poly( $\varepsilon$-caprolactone $)$ \\
\hline PDLLA & poly(DL-lactic acid) \\
\hline PDMS & poly(dimethyl siloxane) \\
\hline PEEK & poly(ether ether ketone) \\
\hline PEG & poly(ethylene glycol) \\
\hline PEG-DA & poly(ethylene glycol)-diacrylate \\
\hline PEO & poly(ethylene oxide) \\
\hline PEOT/PBT & $\begin{array}{l}\text { poly(ethylene oxide terephthalate)-poly(butylene } \\
\text { terephthalate) }\end{array}$ \\
\hline PET & poly(ethylene terephthalate) \\
\hline PET0.4 & PET membranes with $0.4 \mu \mathrm{m}$ pores \\
\hline PETA & pentaerythritol triacrylate \\
\hline PFA & paraformaldehyde \\
\hline PFOCTS & trichloro $(1 \mathrm{H}, 1 \mathrm{H}, 2 \mathrm{H}, 2 \mathrm{H}$-perfluorooctyl)silane \\
\hline
\end{tabular}




\begin{tabular}{|c|c|}
\hline PLA & poly(lactic acid) \\
\hline PLGA & poly(lactic-co-glycolic acid) \\
\hline PMMA & poly(methyl methacrylate) \\
\hline PP & poly(propylene) \\
\hline $\mathrm{PS} \mu \mathrm{M}$ & phase separation micromoulding \\
\hline PTFE & poly(tetrafluoroethylene) \\
\hline PTMC & poly(trimethylene carbonate) \\
\hline PUA & poly(urethane acrylate) \\
\hline PVDF & poly(vinylidene fluoride) \\
\hline PVP & poly(vinyl pyrrolidone) \\
\hline RCOs & rat calvarial osteoblasts \\
\hline RGD & arginine-glycine-aspartic acid \\
\hline SD & standard deviation \\
\hline SEM & scanning electron microscopy \\
\hline SMCs & smooth muscle cells \\
\hline $\mathrm{Sn}(\mathrm{Oct})_{2}$ & stannous octoate \\
\hline TCPS & tissue culture-treated polystyrene \\
\hline $\mathrm{Tg}_{\mathrm{g}}$ & glass transition temperature \\
\hline TGF- $\beta$ & transforming growth factor $\beta$ \\
\hline TIPS & temperature-induced phase separation \\
\hline $\mathrm{TJ}$ & tight junction \\
\hline TMC & (1,3-)trimethylene carbonate \\
\hline TMP & transmembrane pressure \\
\hline UV & ultraviolet \\
\hline VIPS & (water-)vapour-induced phase separation \\
\hline WBC & white blood cell \\
\hline$w t \%$ & weight percentage \\
\hline $\mathrm{w} / \mathrm{w}$ & weight-to-weight \\
\hline $\mathrm{ZO}-1$ & Zonula Occludens 1 \\
\hline
\end{tabular}




\section{- List of publications -}

1. Pasman, T.; Grijpma, D.; Stamatialis, D.; Poot, A. Fabricating porous, photo-crosslinked poly(trimethylene carbonate) membranes using temperature-induced phase separation. Polymers for Advanced Technologies. 2017, 28(10), 1258-1262. DOI: 10.1002/pat.3956.

2. Pasman, T.; Grijpma, D.; Stamatialis, D.; Poot, A. Flat and microstructured polymeric membranes in organs-on-chips. Journal of the Royal Society, Interface. 2018, 15(144), 20180351. DOI: 10.1098/rsif.2018.0351.

3. van Riet, S.; Ninaber, D.K.; Mikkers, H.; Tetley, T.D.; Jost, C.R.; Mulder, A.A.; Pasman, T.; Baptista, D.; Poot, A.A.; Truckenmüller, R.; Mummery, C.L.; Freund, C.; Rottier, R.J.; Hiemstra, P.S. In vitro modelling of alveolar repair at the air-liquid interface using alveolar epithelial cells derived from human induced pluripotent stem cells. Scientific reports. 2020, 10(1), 5499. DOI: 10.1038/s41598-020-62226-1.

4. Pasman, T.; Baptista, D.; van Riet, S.; Truckenmüller, R.K.; Hiemstra, P.S.; Rottier, R.J.; Stamatialis, D.; Poot, A.A. Development of porous and flexible PTMC membranes for in vitro organ models fabricated by evaporation-induced phase separation. Membranes. 2020, 10(11), 330. DOI: 10.3390/membranes10110330.

5. Baptista, D.; Teixeira, L.M.; Birgani, Z.T.; van Riet, S.; Pasman, T.; Poot, A.; Stamatialis, D.; Rottier, R.J.; Hiemstra, P.S.; Habibović, P.; van Blitterswijk, C.; Giselbrecht, S.; Truckenmüller, R. 3D alveolar in vitro model based on epithelialized biomimetically curved culture membranes. Biomaterials. 2021, 266, $120436 . \quad$ DOI: 10.1016/j.biomaterials.2020.120436.

6. Pasman, T.; Baptista, D.; van Riet, S.; Truckenmüller, R.K.; Hiemstra, P.S.; Rottier, R.J.; Hamelmann, N.M.; Paulusse, J.; Stamatialis, D.; Poot, A.A. Development of an in vitro airway epithelial-endothelial cell culture model on a flexible porous poly(trimethylene carbonate) membrane based on Calu-3 airway epithelial cells and lung microvascular endothelial cells. Membranes. 2021, 11(3), 197. DOI: 10.3390/membranes11030197. 


\section{- About the author -}

Thijs Pasman was born in Winterswijk, The Netherlands, on the $5^{\text {th }}$ of April 1990. He finished a Gymnasium program, with a focus on Nature and Health from his secondary school, the Assink Lyceum in Haaksbergen, in 2008. Afterwards, he completed a Bachelor in Biology, with a Biomedical Sciences major, and a Biomedical Sciences Master program, with a focus on regenerative medicine and developmental biology, at the University of Groningen, The Netherlands between 20082013. After his studies, he temporarily worked as a junior researcher at Nano Fiber Matrices B.V. in Groningen, The Netherlands in 2014 before starting his $\mathrm{PhD}$ program in the Biomaterials Science and Technology group at the University of Twente, The Netherlands, in 2015. He developed poly(trimethylene carbonate) membranes for lung epithelialendothelial models, which is described in this thesis. In 2021, he worked in a short XS Biosensing project in the Developmental BioEngineering group at the University of Twente and finished his $\mathrm{PhD}$ program.

Thijs Pasman werd geboren op 5 april 1990 in Winterswijk. In 2008 rondde hij een Gymnasium-opleiding met een Natuur en Gezondheid profiel af aan het Assink Lyceum in Haaksbergen. Daarna voltooide hij een Bachelor in Biologie, met als afstudeerrichting biomedische wetenschappen, en een Masteropleiding Biomedical Sciences, met een focus op regeneratieve geneeskunde en ontwikkelingsbiologie, aan de Universiteit van Groningen tussen 2008-2013. Na zijn studie werkte hij in 2014 tijdelijk als junior onderzoeker bij Nano Fiber Matrices B.V. in Groningen voordat hij in 2015 aan zijn PhD-programma begon bij de groep Biomaterials Science and Technology aan de Universiteit Twente. Hij ontwikkelde poly(trimethyleen carbonaat) membranen voor longepitheel-endotheel modellen. Dit is beschreven in dit proefschrift. In 2021 werkte hij in een tijdelijk XS Biosensing-project in de groep van Developmental BioEngineering van de Universiteit Twente en rondde hij zijn PhD-programma af. 


\section{- Acknowledgements -}

\section{And final words}

After probably the most challenging time of my life up until now, I finally write my last words as a PhD student. The thought of developing and working with organs-on-chips was immediately appealing. Moreover, since I also wanted to broaden my horizon, this project seemed perfect. But I knew that starting out as a pure cell biologist on this project meant that it would be a challenge, and indeed, it has been. I did manage to learn more about cell biology, polymers, membranes, phase separation, organson-chips and much more. For this, I want to thank a lot of people, since I would not have been able to learn so much and finish my PhD project, without them. Moreover, I have been able to make so many friends along the way and too many great memories to mention here.

First of all, I would like to express my gratitude to the graduation committee: Prof. Dr. Jennifer Herek, Prof. Dr. Serge Lemay, Prof. Dr. Robert Passier, Prof. Dr. Ir. Loes Segerink, Prof. Dr. Dirk Grijpma, Prof. Dr. Dimitrios Stamatialis and Dr. André Poot from the University of Twente, and Prof. Dr. Roman Truckenmüller from Maastricht University, Prof. Dr. Pieter Hiemstra from Leiden University and Dr. Loredana De Bartolo from the Institute on Membrane Technology of the National Research Council of Italy. Thank you for taking the time and effort to evaluate my thesis and being part of my committee. I had the chance to work closely with some of you, either within the consortium or the Biomaterials Science and Technology (BST) group in which this project took place. I have also been present at presentations from many of you in courses, conferences or elsewhere. I am also grateful for being able to learn from you in those ways. 
Besides my committee, I want to extend my thanks to the Longfonds and the University of Twente for making this project possible and providing me with the chance, facilities and support to do this research.

On a more personal level, I want to sincerely thank Dirk, Dimitrios and André, my supervisors, again. You gave me the position and chance in your BST group to do this project. The three of you always gave me the necessary knowledge, encouragement and nudge in the back to push forward. Having this many supervisors meant that my work was always viewed from many different angles, which was insightful and very educational, but was also challenging at times, since it sometimes meant having three different views on something. I remember going from office to office to find a definition for a membrane that satisfied all of us. You taught me many valuable lessons about all aspects of research, and I greatly value you for that as well as for the more informal times we spent together on consortium meetings, conferences and outings, as well as travelling to get there. I cannot thank you enough!

Next, I would like to thank the consortium of which this project was a part. The goal of the consortium was to create: Microengineered 3D analogues of alveolar tissue for lung regeneration. To establish this, researchers from the Universities of Leiden, Rotterdam, Maastricht and Twente worked together. Roman, Pieter, Robbert, Stefan, Sander and Danielle, thank you for the pleasant co-operation, critical view and support on my work and papers. The atmosphere was always professional, yet informal, which I greatly appreciated. I also appreciate the times that I could visit your universities where you provided the necessary practical support, such as my visits to the confocal microscope in Rotterdam. I also enjoyed spending time together at different conferences and other events.

I would then like to thank Jos and Naomi from the Biomolecular Nanotechnology group of the University of Twente. Although you left the BST group and started a new group somewhere else in the university, you 
gave me the opportunity to do very important measurements of my cell culture models, for which I am very grateful. Moreover, it was nice to spend a bit more time with you after you left the BST group.

Then I want to extend my thanks to Eef, Lotte, Raquel and Olja. You showed a great interest in my project, which was very motivating, and you put your trust in me as your supervisor for a project in your studies. I want to thank you for that. I hope I have been able to teach you or help you learn, as I have been able to learn from working with you. Although not all of the work that we put in this project made the final cut in this thesis, your work gave me the tools and knowledge to finish the project.

Also, I want to thank my paranymphs, Marcel and Odyl. You have not only supported me in the period before my defence, but have been great supports over the years. Odyl, you have been an office mate since day one, and since we started roughly at the same time, we could share many of the same experiences. You were also a great help at the membrane side of things. Besides, I greatly enjoyed your company outside of work and at conferences such as the one in Vienna.

Marcel, after an internship, you stayed to become a PhD student in the group. Since you were working with cells and fabricated your own constructs, we would often see each other in the cell culture lab and relate to each other, mostly concerning challenges with cells, chips, etc. But we also met outside of work and, for example, at the ESCDD in Egmond aan Zee. Good luck with finishing your thesis soon!

Although a research group has a lot of temporary members, it also has a fixed core, which included my supervisors, but also a group of individuals which kept the group together and supported me in many ways, either directly or through presentations, discussions, etc. Because of this, I would like to thank Karin Hendriks, Marc, Lydia, Zlata, Jai and Hetty. I especially want to thank a couple of you whom I worked with more closely. Karin, you have been a support in all kinds of things in terms of administration, registration and much more, and especially in 
the last half year or so with arranging things for the defence and thesis. Throughout the years, I have seen people write about you as the 'mother' and 'wizard' of the BST group, and I can see why. Marc, you have been extremely valuable on the labs concerning polymers, chemicals and chemical reactions and simply working on a chemical lab as a whole. Besides that, I very much enjoyed the talks we had about a lot of other things while working on the lab and outside of it. I particularly remember the bus-trip to the Scheepvaartmuseum in Amsterdam during the ESCDD conference in Egmond aan Zee. Lydia, despite that you only worked parttime, you were always there and always helpful when it came to things concerning membranes, such as the water flux measurements, wafers, etc., but also in the ML-II lab concerning cell culture and how things went in that lab. I also remember the trust of you and Hetty in me, particularly the time that the lid of the nitrogen tank with cells was damaged and not closed properly. Although I was the last person who registered using the tank, you did not believe that I did that. Thank you both for that trust.

Zlata, thank you for arranging everything about the ordering of products and entrusting me, along with a few others, with that task when you were not present at the university. I also appreciate that you were always thankful when I came to you when something was running low or was not present in the general stock. That also gave the opportunity to talk, which I appreciated as well.

Then there are the current $\mathrm{PhD}$ students and post-docs of the group which I would like to thank. Ahmed, you were always so calm and never looked frustrated. Also, I guess we had a running gag in the ML-II lab where you would always ask something like: "Are you here again?" to which I would reply: "Yes, but so are you."

Nazia, although we only briefly shared an office due to the pandemic, I did enjoy it while it lasted. And recently, you became a mother to your newborn daughter Yusra. I wish you and your family a lot of happiness together. Marleen, it was nice to basically pass down the torch of ZH283 to you and Marc, almost literally in the shape of a weed torch for polymer synthesis. It was great to see your devotion to more attention on working 
more environmentally friendly in the lab. Kunal, congratulations with the start of your PhD project. You were always kind, and very polite during your internship. You deserve the position.

I wish you all the best in completing your projects.

Besides the current members of the Zuidhorst, there have also been so many great people who have left the group before me. Thank you for all the great memories and giving me reminders that there will be a life beyond a PhD project. First of all, I will mention Ilaria, the Godmother, the office boss, she who eats like a pork, as you described yourself or how others called you. Odyl, you and me were the rookies of the group when I started. Your liveliness was great and spending time with you and Save, for example in Vienna, was always fun. And recently, you became parents to your newborn daughter Agnese. I wish you two, Agnese and Nina all the best.

Bas and Mike, you were two completely different lab chiefs, almost to the point of a good cop/bad cop routine, great stuff! Also, among great memories, I have not forgotten about the song 'Ring of Fire' during my first conference in Utrecht with you. Vincent, thank you for helping me make my first membranes and Zhengchao, thank you for helping me synthesise my first PTMC. Also, you, Jia and Aysun, thank you for the nice talks in and around the lab and the great times we had at several different conferences, such as the NBTE, and also outside of work.

I also thank the BST (Bio)Artificial Organs cluster. The cluster meetings together were always fun. Also, I will probably never forget the pictures you put on the door of the lab with me as David Beckham and many of you as the Spice Girls. Iris, you were first a PhD student at the group, then came back as a post-doc, working on constructs in which I could help you get started. After you finished that project, you considered and accepted me for your XS Biosensing project, which I greatly appreciate. Thank you for the trust and the great times together. Katarzyna, Natalia, DooLi, the neighbours of our office. Thanks for the support in the lab concerning membranes, coatings and cell culture, and fun during summer school, conferences and outside of work. Denys, thanks for considering me man 
enough to join your work-outs with Bas, even though I never actually joined. What was it again: exercise until you can't do any more, plus two? Dhadhang or 'broer', as we started calling each other. Thank you for always having a smile on your face and making me smile as well!

Lena, Ruchi, although you left to form your own group, you helped me a lot during the project together with Iris. Thank you for that as well as the nice chats in and around the labs!

Besides these people, there are still so many people I also want to thank: Anita, Deby, Jonas, Praneeth, Anna, Tao, Stijn, Frits, Nick, Piet, Duco, Magda, Agnieszka, Karin Postma, Dwi, Pia, Tony, Bade, Gert-Jan, Bram and Barbara, Rachel, Jonathan, Anne Boerema-de Munck and Karin Roelofs and Dennis from 20MedTherapeutics. I enjoyed the talks about work or otherwise, and our time together on the labs, but also outside of them on outings and such! I also want to thank all the students that have been or are still in the BST group, for your enthusiasm, ambition and lively spirits, which I greatly enjoyed. Besides the BST members, I would also like to thank all the members from the DBE, NBP, AST and AMBER groups that I have come to know throughout the years, whether it was in the labs during my $\mathrm{PhD}$, working part-time in the $\mathrm{DBE}$ group or meeting you on conferences or outside of work. Unfortunately, it would simply take too much space to name all of you, but I would like to say that you all enriched my $\mathrm{PhD}$ experience and enjoyment further.

Daarnaast wil ik ook graag mijn familie en vrienden bedanken. Allereerst Arno en Giny, bedankt voor al het plezier, alle steun en alle wijsheid die jullie mij gedurende mijn hele leven, maar vooral ook de afgelopen jaren hebben gegeven. Jullie hebben me op werkelijk alle manieren gesteund en daar kan ik jullie niet genoeg voor bedanken! We hebben ontzettend veel mooie momenten gehad samen tot nu toe, maar er is één ding in het bijzonder dat mij bij is gebleven. Na mijn studie namen jullie me zo weer een paar jaar in huis. Dat was een stuk gezelliger dan in mijn eentje wonen. Veel mensen begrepen niet dat ik na mijn studie zo makkelijk weer bij mijn ouders introk. Ik heb daar echter nooit problemen mee 
gehad en voel me nog steeds altijd thuis bij jullie. Dit zegt voor mij al genoeg over hoeveel geluk ik met jullie heb!

Daarnaast bedank ik ook jullie, Mandy, Bertram en sinds ruim een jaar ook Steyn. Mandy, jou ken ik natuurlijk al mijn hele leven als mijn 'kleine zusje', maar inmiddels ben je nu zelfs moeder en hebben jullie met zijn drieën een mooi gezin. Jullie hebben me ook altijd gesteund, wat ik erg waardeer!

We hebben met zijn allen mooie tijden samen beleefd, zoals op vakantie, en ik hoop dat er nog vele mooie momenten samen zullen zijn.

Steun en interesse ontving ik ook van de rest van de families, waarvoor ik jullie erg wil bedanken. Het geeft een heel fijn gevoel te weten dat er zoveel mensen zijn bij wie de deur altijd voor je open staat!

Daarnaast wil ik de familie Rosendaal ook bedanken dat jullie mij zo hartelijk hebben ontvangen toen ik Lisa drie jaar geleden ontmoette! Ook bij jullie ontbrak het vanaf het begin niet aan interesse en steun.

In het bijzonder bedank ik jullie, Annelies, Peter, Rick, Tom, Jolien en sinds ruim een jaar ook Milou, voor het warme onthaal in jullie gezin. Ook wil ik jullie, Annie en Gerrit Rosendaal, oftewel opa en oma, speciaal bedanken. Jullie hebben mij met open armen opgenomen als kleinzoon. Ondanks dat mijn andere grootouders niet meer leven, voelt het weer als vroeger bij jullie. Ik had graag mijn andere grootouders ook nog bij mijn verdediging gehad, aangezien zij ook altijd veel interesse hadden in alle kleinkinderen, waaronder mij en mijn project. Daarom vind ik het erg bijzonder dat ik mijn PhD af kan sluiten met jullie in mijn leven.

Ook wil ik Kevin, Leroy, Nils, Wytze, Dennis en Roy bedanken voor jullie vriendschap. We hebben een mooie groep bij elkaar en ik ben blij er deel van uit te mogen maken. En de groep is in de loop der jaren gegroeid met Susanne, Sem en Cas, Sanne en Jurre, Zilyara, Anett en Lisa. Hierdoor moesten we een beetje volwassen worden, maar kunnen we toch nog steeds ouderwets lol hebben zoals op de middelbare school. Allemaal bedankt voor de geweldige vriendschap! 
Bovendien bedank ik ook Rudy en Barbera. Ik heb jullie leren kennen als goede vrienden van Lisa, maar inmiddels beschouw ik jullie ook als goede vrienden van mezelf en ik hoop dat we nog vaak samen op pad gaan!

Een beetje ongewoon misschien, maar ik wil toch ook Storm, Louki, en alle vogels bij mijn ouders, waar ik veel voor heb mogen zorgen, even noemen. Jullie hebben mij heel veel ontspanning en rust gegeven en jullie zijn een enorme bron van plezier.

En als laatste bedank ik jou, Lisa. Ondanks dat je me in de drukste en meest uitdagende tijd van mijn leven hebt leren kennen, ben je altijd geduldig geweest, heb je altijd in mij en ons geloofd en heb je me geweldig gesteund op elke mogelijke manier! Ik kan je daar niet genoeg voor bedanken en besef dat ik enorm veel geluk heb met jou. Nu het project ten einde is kunnen we voor het eerst eindelijk echt eens een normale Kerst of vakantie hebben, zonder dat er iets is van mijn project, zoals de cellen. Misschien hebben we dan ook eindelijk eens de gelegenheid en tijd om eens te ruziën, want dat is in de drie jaar dat we elkaar kennen nog steeds niet gelukt. Maar als je daar nog jaren mee wilt wachten, is dat ook goed. Even serieus. Jij hebt altijd verder kunnen kijken dan mijn $\mathrm{PhD}$ project en altijd een mooie toekomst samen gezien. Eenzelfde toekomst had en heb ik nog steeds voor ogen, en die ga ik nu heel graag samen met jou tegemoet!

Finally, although these are by no means official postulates, I want to end with words from other people or characters, mostly found in popular media. These words are applicable to science, life in general or both. They have been meaningful to me and will be in the future and I hope some of them may prove useful to you as well.

Ten slotte wil ik, hoewel het op geen enkele manier officiële stellingen zijn, eindigen met woorden van andere mensen of personages, meestal te vinden in populaire media. Deze woorden zijn van toepassing op de wetenschap, het leven in het algemeen of beide. Ze zijn waardevol 
geweest voor mij en zullen dat in de toekomst ook blijven. Ik hoop dat sommige ervan ook nuttig voor jou kunnen zijn.

"Life finds a way."

"Het leven vindt een weg."

Dr. Ian Malcolm, Jurassic Park and Jurassic World

"What makes us such unique creatures is knowing the scope and power of what we are up against, and still believing that we can win."

"Wat ons zulke unieke wezens maakt, is dat we de omvang en kracht kennen van waar we tegenover staan, en nog steeds geloven dat we kunnen winnen."

Dr. Ian Malcolm, Jurassic Park and Jurassic World

"I'm going to beat fantasy with science."

"Ik ga fantasie verslaan met wetenschap."

Senku Ishigami, Dr. Stone

"I'm going to use the power of science to rescue every single person."

"Ik ga de kracht van wetenschap gebruiken om iedereen te redden."

Senku Ishigami, Dr. Stone

"Science levels the playing field for everyone."

"Wetenschap geeft iedereen gelijke kansen."

Senku Ishigami, Dr. Stone

"With great power comes great responsibility."

"Met grote kracht komt grote verantwoordelijkheid."

Ben Parker, Spider-man

"All we have to decide is what to do with the time that is given to us."

"Het enige wat we hoeven te beslissen is wat we doen met de tijd die ons wordt gegeven."

Gandalf, The Lord of The Rings 
"It is the small, everyday deeds of ordinary folk that keeps the darkness at bay. Simple acts of kindness and love."

"Het zijn de kleine, alledaagse daden van gewone mensen die de duisternis op afstand houden. Eenvoudige daden van vriendelijkheid en liefde."

Gandalf, The Hobbit

"A thing isn't beautiful because it lasts."

"Iets is niet mooi omdat het blijft bestaan."

Vision, Avengers: Age of Ultron

"Anything in this world that is worth having, doesn't come easy."

"Alles in deze wereld dat de moeite waard is om te hebben, krijg je niet zomaar."

Dr. Bob Kelso, Scrubs

"Yesterday is history, tomorrow is a mystery, but today is a gift. That's why it is called the present."

Master Oogway, Kung Fu Panda

"We don't have to know what tomorrow holds! That's why we can live for everything we're worth today!"

"We hoeven niet te weten wat morgen in petto heeft! Daarom kunnen we vandaag leven voor alles wat we waard zijn!"

Natsu Dragneel, Fairy tail

"Though our paths may have diverged, you must continue to live out your life with all your might, you must never consider your own life to be something insignificant, and you must never forget about your friends who loved you."

"Hoewel onze wegen misschien van elkaar zijn gescheiden, moet je je leven met al je macht blijven leven, je eigen leven nooit als iets onbelangrijks beschouwen, en je vrienden die van je hielden nooit vergeten." 
Finally, I would like to add two myself, since I have come to realise in the last years that regret is one of the most important things you should try to avoid in life. Moreover, the question "What if..?" should be handled with care:

",What if..?' is a devastating question for people with regrets or worries, but a never-ending wonder for dreamers. Try to be a dreamer."

And ",What if..?' should only be asked for what you can gain, not for what you can lose."

I hope you can chase your dreams, live your life to the fullest and without regrets, and I hope you have a lot of fun while doing that. Thank you all again, hope to see you again soon, and I wish you all the best.

Ten slotte zou ik er zelf graag twee willen toevoegen, aangezien ik de afgelopen jaren ben gaan beseffen dat spijt één van de belangrijkste dingen is die je in het leven moet proberen te vermijden. Bovendien moet de vraag "Wat als..?" met zorg worden behandeld:

",Wat als..?' is een verwoestende vraag voor mensen met spijt of zorgen, maar een oneindig wonder voor dromers. Probeer een dromer te zijn."

En ",Wat als..?' moet je alleen vragen voor wat je kunt behalen, niet voor wat je kunt verliezen."

Ik hoop dat je je dromen kunt najagen, je leven ten volle en zonder spijt kunt leven, en ik hoop dat je veel plezier zult hebben terwijl je dat doet. Nogmaals bedankt allemaal, ik hoop jullie snel weer te zien, en ik wens jullie heel veel geluk en het allerbeste. 
- 261 - 
- 262 - 



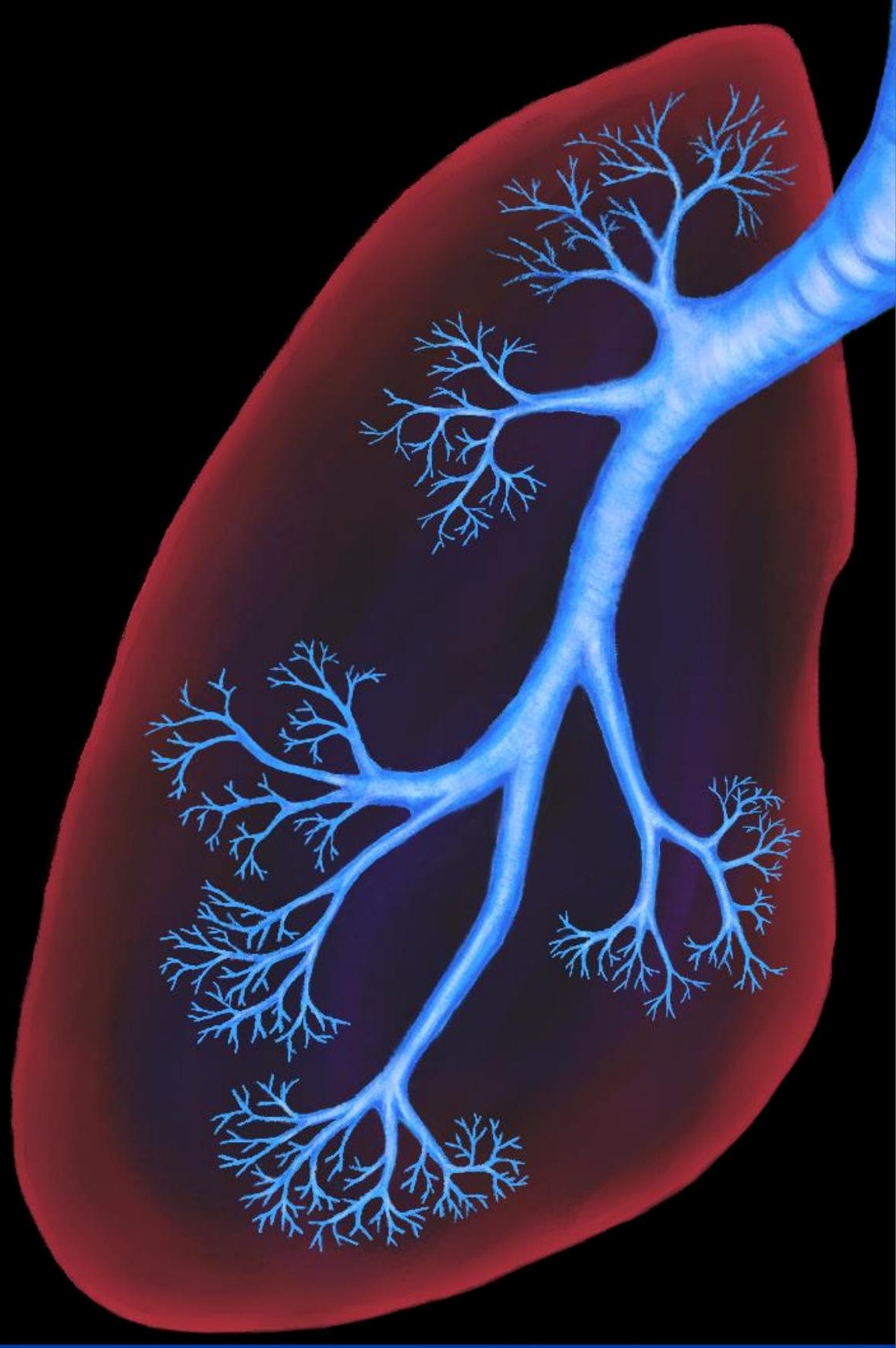

